\title{
Modified delphi investigation of exercise science in physical education teacher education
}

\author{
Sean M. Bulger \\ West Virginia University
}

Follow this and additional works at: https://researchrepository.wvu.edu/etd

\section{Recommended Citation}

Bulger, Sean M., "Modified delphi investigation of exercise science in physical education teacher education" (2004). Graduate Theses, Dissertations, and Problem Reports. 2625.

https://researchrepository.wvu.edu/etd/2625

This Dissertation is protected by copyright and/or related rights. It has been brought to you by the The Research Repository @ WVU with permission from the rights-holder(s). You are free to use this Dissertation in any way that is permitted by the copyright and related rights legislation that applies to your use. For other uses you must obtain permission from the rights-holder(s) directly, unless additional rights are indicated by a Creative Commons license in the record and/ or on the work itself. This Dissertation has been accepted for inclusion in WVU Graduate Theses, Dissertations, and Problem Reports collection by an authorized administrator of The Research Repository @ WVU.

For more information, please contact researchrepository@mail.wvu.edu. 
Modified Delphi Investigation of Exercise Science in Physical Education Teacher Education

Sean M. Bulger

Dissertation submitted to the School of Physical Education at West Virginia University in partial fulfillment of the requirements for the degree of

\author{
Doctor of Education in \\ Physical Education Teacher Education
}

\author{
Lynn Housner, Ph.D., Chair \\ Carl P. Bahneman, Ph.D. \\ Randall Bryner, Ed.D. \\ Richard T. Walls, Ph.D. \\ Robert L. Wiegand, Ed.D. \\ School of Physical Education
}

\author{
Morgantown, West Virginia \\ 2004
}

Keywords: Professional Preparation, Teacher Education

Copyright 2004 Sean M. Bulger 


\section{ABSTRACT \\ Modified Delphi Investigation of Exercise Science in Physical Education Teacher Education}

Sean M. Bulger

As physical education teacher educators experiment with alternative pedagogical approaches to bridge the gap between subdisciplinary theory and professional practice, the need for enhanced multi-disciplinary research and communication continues. The purpose of this study was to determine the critical exercise science competencies, and associated instructional methods, that are recommended for inclusion in the physical education teacher education undergraduate curriculum. An initial list of theoretical and applied exercise science competencies was constructed using the knowledge, skills, and abilities recommended by the American College of Sports Medicine for individuals working in the field of exercise science. The two-round, modified Delphi procedure involved the repeated circulation of this questionnaire to a small panel of content experts. Experts in the field of physical education, including exercise science specialists, physical education teacher educators, and physical education teachers, were sampled to determine which theoretical and applied exercise science competencies are critical for the preparation of prospective physical educators. The Delphi panel members were required to rate each questionnaire item in terms of its theoretical importance and pedagogical relevance using a five-point Likert scale. The data collected during the second round of questioning were employed to provide a final measure of consensus regarding the critical strength of each exercise science competency. An item had to meet the following criteria in order to be considered of both critical importance and relevance: (a) the item received a mean rating of at least four or higher in the areas of importance and relevance; and (b) the item received at least $75 \%$ of all individual ratings at the four level or higher. Any item that failed to meet these criteria was considered to be non-critical. The Delphi panel members were also asked to complete an addendum survey concerning their recommendations regarding the four most effective instructional methods for the delivery of exercise science to prospective physical education teachers. The results of this scholarly process, which includes a final list of recommended exercise science competencies and instructional methods, have provided a conceptual framework upon which physical education teacher educators can make future curricular decisions in the area of exercise science. 


\section{DEDICATION}

This manuscript is dedicated to the teachers who inspired me to pursue a career in higher education. Most notably to my mother, Harriett Ann Bulger, whose passion for teaching and creativity is overshadowed only by her uncompromising commitment to the students she serves. 


\section{ACKNOWLEDGMENTS}

The members of my Delphi panel, whose expertise served as the inspiration for this study, deserve a very sincere and special acknowledgement. Their commitment to the completion of this research project is now, and forever shall be, greatly appreciated. I also acknowledge my Chair, Lynn Housner, and his fellow committee members, Carl Bahneman, Randall Bryner, Richard Walls and Robert Wiegand, whose proficiency and mentorship have played an instrumental role in my continued education and professional development. I would like to thank my friends and colleagues, Michael Graves, Derek Mohr, Richard Rairigh, Darren Robert, and Scott Townsend, who helped to make my time at West Virginia University such a rewarding and valuable experience. Most importantly, I must acknowledge my wife Donna for her tireless support and patience. 


\section{TABLE OF CONTENTS}

\section{CHAPTER I}

\section{INTRODUCTION AND STATEMENT OF THE PROBLEM}

Introduction

The Problem

Statement of the Problem

Scope of the Study

Basic Assumptions

Limitations of the Study

Definition of Terms

Significance of the Study

\section{CHAPTER II}

\section{REVIEW OF LITERATURE}

Children's Physical Activity and Health

The Health and Physical Activity Connection 8

Cardiovascular and Metabolic Health 8

Musculoskeletal Health $\quad 9$

Immune System Health $\quad 10$

Psychosocial Health $\quad 10$

Childhood Physical Activity and Adult Health $\quad 10$

Professional Recommendations for Children's Physical Activity 11

American Academy of Pediatrics $\quad 11$

American College of Sports Medicine $\quad 12$

American Heart Association $\quad 12$

International Consensus Conference on Physical Activity 12

National Strength and Conditioning Association 13

National Association for Sport and Physical Education $\quad 13$

Physical Activity in School Physical Education Programs 14

Child and Adolescent Trial for Cardiovascular Health (CATCH) 15

Heart Smart 16

Sports, Play, and Active Recreation for Kids (SPARK) 16

$\begin{array}{ll}\text { Implications for PETE Programs } & 16\end{array}$

$\begin{array}{ll}\text { Bridging the Gap Between Theory and Practice in PETE } & 17\end{array}$

$\begin{array}{ll}\text { Historical Perspectives } & 17\end{array}$

$\begin{array}{ll}\text { The Gap Between Theory and Practice } & 18\end{array}$

The Gap Between Theory and Practice in Exercise Science 20

$\begin{array}{ll}\text { Course Content } 20 & 20\end{array}$

Instructional Methods $\quad 22$

Proposed Solutions for Bridging the Gap 23

Delphi Method: A Tool for Multi-Disciplinary Research and Communication 25

Delphi Method Objectives $\quad 25$

Delphi Method Protocol 27

Delphi Panel 27

Questionnaire Design and Administration 27 
Asynchronous and Anonymous Participation 28

Supervision and Feedback 28

Group Consensus 29

Advantages of the Delphi Method 29

Limitations of the Delphi Method 29

$\begin{array}{ll}\text { Summary } & 30\end{array}$

\section{CHAPTER III METHDOLOGY}

$\begin{array}{ll}\text { Participants } & 31\end{array}$

Research Design $\quad 32$

$\begin{array}{ll}\text { Instrumentation } & 32\end{array}$

Administrative Procedures 33

Selection of Participants $\quad 33$

Round I Procedures $\quad 34$

Round II Procedures $\quad 34$

Data Analysis $\quad 34$

Exercise Physiology Critical Concepts $\quad 35$

Exercise Physiology Instructional Strategies $\quad 35$

\section{CHAPTER IV RESULTS}

Anatomy and Biomechanics 36

Exercise Physiology 36

$\begin{array}{ll}\text { Human Development and Aging } & 37\end{array}$

$\begin{array}{ll}\text { Pathophysiology and Risk Factors } & 37\end{array}$

$\begin{array}{ll}\text { Human Behavior and Psychology } & 37\end{array}$

$\begin{array}{lr}\text { Health Appraisal and Fitness Testing } & 38\end{array}$

Safety, Injury Prevention, and Emergency Care 38

$\begin{array}{ll}\text { Exercise Programming } & 39\end{array}$

Nutrition and Weight Management 39

$\begin{array}{ll}\text { Program Management and Administration } & 40\end{array}$

$\begin{array}{ll}\text { Instructional Methods } & 40\end{array}$ 
$\begin{array}{ll}\text { Teacher-directed Instruction } & 40\end{array}$

Active Learning $\quad 41$

Lab Component 41

Experiential Learning $\quad 42$

Technology Integration $\quad 42$

Alternative Curricular Models 43

CHAPTER V DISCUSSION

Limitations of Delphi Method 44

Exercise Science in PETE: Review 45

Exercise Science in PETE: Practical Implications 46

Exercise Science Course Content 46

Exercise Science Instructional Methods $\quad 50$

REFERENCES 


\section{LIST OF TABLES}

Table 1 Sample Applications of Delphi Method in Physical Education Research 66

Table 2 Exercise Science in PETE Delphi Panel Members $\quad 67$

Table 3 Category I - Competencies Related to Anatomy and Biomechanics 68

Table 4 Category II - Competencies Related to Exercise Physiology 70

Table 5 Category III - Competencies Related to Human Development and Aging 73

Table 6 Category IV - Competencies Related to Pathophysiology/Risk Factors 74

Table 7 Category V - Competencies Related to Human Behavior and Psychology 76

Table 8 Category VI - Competencies Related to Health Appraisal/Fitness Testing 78

Table 9 Category VII - Competencies Related to Safety and Emergency Care 80

Table 10 Category VIII - Competencies Related to Exercise Programming 82

Table 11 Category IX - Competencies Related to Nutrition/Weight Management 86

Table 12 Category X - Competencies Related to Program Management 88

Table 13 Final List of Critical Exercise Science Competencies $\quad 89$

Table 14 Delphi Panel Member Responses Regarding Non-Critical Exercise 94 Science Competencies 


\section{LIST OF FIGURES}

Figure 1 Recommended Instructional Methods for Delivering Exercise Science Content in PETE 108

Figure 2 General Summary of Delphi Panel Findings Regarding Exercise Science Content and Recommended Instructional Methods in PETE

Figure 3 Overview of a Conceptual Framework for the PETE Curriculum

Figure 4 Summary of Theoretical Exercise Science Competencies in PETE

Figure 5 Summary of Applied Exercise Science Competencies in PETE 


\section{LIST OF SYMBOLS}

Symbols Definition

\begin{tabular}{ll}
\hline$M$ & Mean (arithmetic average) \\
$n$ & Number in a sub-sample \\
$\%$ & Percentage \\
$S D$ & Standard deviation \\
\hline
\end{tabular}




\section{CHAPTER I \\ INTRODUCTION AND STATEMENT OF THE PROBLEM}

Course work in the area of exercise science is recognized as an important foundational component of the physical education teacher education (PETE) curriculum (National Association for Sport and Physical Education, 1998a). It is generally assumed that the theoretical and applied exercise science concepts addressed within the PETE curriculum directly contribute to the prospective teacher's ability to effectively facilitate the development of the psychomotor, cognitive, affective, and fitness characteristics that enable a child to enjoy a physically active lifestyle into adulthood. The legitimacy of this basic assumption has been questioned in the literature, however, and there is evidence to suggest that the delivery of exercise science-related content has been a problematic issue for some PETE programs (Barnett \& Merriman, 1994; Kelley \& Lindsay, 1977, 1980; Miller \& Housner, 1998). More specifically, the course content and instructional strategies commonly associated with the exercise science component of the PETE curriculum are two possible areas of concern.

While courses in exercise science and physical fitness have been previously identified as a primary source of knowledge for undergraduate PETE students (Mood, 1971), questions regarding the appropriateness of the associated course content remain. Relatively few studies, however, have directly investigated the role of exercise science within the PETE curriculum and the relevance of the associated course content (Barnett \& Merriman, 1994; Kelley \& Lindsay, 1977, 1980; Miller \& Housner, 1998). Kelley and Lindsay conducted two studies to determine the knowledge obsolescence of pre-service and in-service physical educators in several academic domains, including exercise physiology. The results of these studies suggested that research developments in exercise physiology at that time were not being used to effectively update the related course content of the involved PETE programs.

Similarly, Barnett and Merriman (1994) compared the health-related physical fitness knowledge of physical education and non-physical education majors. The participating physical education majors demonstrated a significantly greater understanding of physical fitness than the non-physical education majors. The prospective physical educators exhibited incremental increases in physical fitness knowledge as they progressed through the first three years of the teacher education curriculum, but there were no significant differences between the junior and senior grades. In light of these findings, Barnett and Merriman called for additional investigation regarding (a) the prerequisite level of fitness knowledge needed to teach physical education, (b) the process by which prospective physical educators acquire fitness knowledge, and (c) the ability of prospective physical educators to apply learned fitness concepts in an applied setting.

Miller and Housner (1998) assessed the health-related physical fitness knowledge of pre-service physical educators, in-service physical educators, and graduate students in physical education and exercise physiology. The findings indicated that the participating pre-service and in-service physical educators demonstrated an inadequate knowledge of health-related physical fitness. The poor performance of the pre-service group in this study may be related to a number of factors including: (a) the educational background of the participants prior to enrollment in the PETE program, (b) the nature of the PETE curriculum, and (c) the lack of adequate educational experiences in the area of health-related physical fitness. Miller and Housner also suggested that PETE programs should closely evaluate the content validity, pedagogical relevance, and instructional quality of exercise physiology courses for preservice physical educators.

Although limited, the previously described studies (Barnett \& Merriman, 1994; Kelley \& Lindsay, 1977, 1980; Miller \& Housner, 1998) support the position that there are several questions associated with the appropriateness of the exercise science content that is typically included in the PETE curriculum.

Locke (1990) proposed that practicing physical educators fail to use subdisciplinary knowledge because it 
is basically irrelevant to their work in school settings. It is entirely possible that "the model of the rational teacher who bases instructional decisions on research-based knowledge from the foundational sciences does not describe how most teaching gets done in physical education classes" (Locke, 1990, p. 137). Corbin (1993) also contended that subdisciplinary knowledge is of little practical value if practitioners are unable to effectively apply it to the teaching of physical education. In many respects, research in the subdisciplines of physical education appears to have fragmented and intellectualized the field while neglecting the more practical issue of application (Greendorfer, 1987b; Hellison, 1991). McKenzie and Sallis (1996) argued "the trend to require potential physical education specialists to take more theoretical and scientific courses while reducing their exposure to instructional methods, program development and assessment, and physical activities is not necessarily positive" (p. 237). Physical educators who complete a more theoretically oriented plan of study may be "better prepared to teach sedentary, cognitive-focused classes than they are to teach students to be physically active" (McKenzie \& Sallis, 1996, p. 237-238).

The instructional methods associated with exercise science courses may also contribute to the problematic gap between theory and practice. Barnett and Merriman (1994) proposed that PETE programs have not provided sufficient integration between the learning of physical fitness concepts in the classroom and their eventual application in a school-based context. If students are not provided with instructional methodologies that are specifically designed to relate theory to practical problems, they may regard the theory as being irrelevant and educational practice remains largely unaffected by the continually expanding body of subdisciplinary knowledge (Bain \& Poindexter, 1981).

Dodds (as cited in Van Donselaar \& Leslie, 1990) reported that introductory exercise physiology course enrollment traditionally includes students from a variety of majors who have different intentions for the eventual practical application of the involved course content. Course enrollment frequently includes students who are majoring in physical education, athletic training, exercise physiology, athletic coaching, and physical therapy. It is unlikely that the unique instructional needs of each student can be satisfactorily addressed in a course with this type of diverse enrollment (Van Donselaar \& Leslie, 1990). Furthermore, the introductory anatomy and physiology courses required in many PETE programs are likely to be (a) taught outside of the PETE program, (b) instructed by non-teacher educators, and/or (c) influenced little by PETE faculty regarding course content and instructional methods (Verner, 1991). While subdisciplinary specialists are likely to maintain a more complete understanding of the involved subject matter, they may lack the knowledge and ability to integrate the essential pedagogical concepts that make the theory to practice transition a workable proposition. Bain and Poindexter (1981), for example, suggested that specialists in the subdisciplines are often unwilling to dilute their theoretical course content with issues of practical application. This reluctance to directly address theory and application may be further compounded by "the discipline-based faculty member's inexperience in public school teaching, and in teaching sports as well" (Bain \& Poindexter, 1981, p. 40). The diverse nature of the course enrollment, associated time constraints, and instructor characteristics may interact to produce an educational environment that does not readily facilitate the translation of theory into the practice of teaching physical education.

To counter the problems associated with the gap between theory and practice, a variety of methods have been proposed in the related literature. In order to narrow the gap between theory and practice, it is generally accepted that the course content, instructional methodology, and roles of both the student and instructor must be altered within PETE programs (Collier \& O'Sullivan, 1997). It has been suggested that PETE programs must employ instructional methods that foster the development of pedagogical content knowledge that enables students to use subdisciplinary knowledge when resolving practical educational problems (Bain \& Poindexter, 1981). The learner must become a more active participant in the teachinglearning process, while the teacher educator must bear the increased responsibility of disseminating current theoretical information in combination with accepted pedagogical practice (Loughery, 1985). 
Of particular interest, Van Donselaar and Leslie (1990) conducted a survey of PETE programs to investigate the current and recommended practices for preparing physical education teachers in exercise physiology. Results demonstrated that a considerable gap exists between the current and recommended instructional strategies for delivering exercise physiology content to prospective physical educators. The findings support the need for "increased attention to physical education teaching applications, reduced reliance on student initiative in making such applications, improved preparation for physical education majors to teach physical fitness concepts, and more collaboration between EP [exercise physiology] and TE [teacher education] personnel" (Van Donselaar \& Leslie, 1990, p. 215).

Other proposed solutions related to the study of exercise science include: (a) The formation of professional committees consisting of subdisciplinary experts during curricular revision (Ross, 1981); (b) the use of the Basic Stuff Series (Kneer, 1981) in teacher education (Bain \& Poindexter, 1981; Loughery, 1985), (c) the design and implementation of culminating experiences that enhance a student's ability to integrate subdisciplinary knowledge in a variety of contexts (Robertson \& Heyden, 1985), (d) the increased focus on developmental rather than adult exercise physiology concepts (Bulger, Mohr, Carson, Robert, \& Wiegand, 2000; Karper, 1997; Miller \& Housner, 1998), (e) the continued evaluation of instructor qualifications and course content (Bulger, Mohr, Carson et al., 2000; Miller \& Housner, 1998; Verner, 1991), and (f) the infusion of a health-related fitness curricular thread or strand within the PETE curriculum (Bulger, Mohr, Carson, \& Wiegand, 2001; Feingold, 1994). Furthermore, Dodds (as cited in Van Donselaar \& Leslie, 1990) recommended the use of separate or team-taught exercise physiology labs for physical education majors. Additionally, Bain and Poindexter (1981) suggested emphasizing the application of pedagogical concepts within subdisciplinary courses, integrating subdisciplinary concepts into professional development courses, and developing new courses that focus specifically on bridging the gap between theory and practice.

Although numerous strategies have been proposed in the literature, bridging the gap between subdisciplinary theory and practical application remains a challenging task for PETE students and instructors (Robertson \& Heyden, 1985). There is a specific need for further research concerning the content and instructional methods employed within PETE programs concerning the delivery of exercise science content and its eventual application in school-based settings. This study contributed to the current body of knowledge by addressing the following research questions: (a) What theoretical and applied exercise science competencies do physical education teacher educators, exercise science specialists, and practicing physical educators consider essential in the undergraduate preparation of prospective physical education teachers? (b) What instructional methods do physical education teacher educators, exercise science specialists, and practicing physical educators recommend for the professional preparation of physical education teachers in the content area of exercise science?

\section{The Problem}

The purpose of this study was (a) to generate a consensus of expert judgment regarding the critical theoretical and applied exercise science competencies that prospective physical educators need to learn within the PETE curriculum and (b) to generate a list of recommended instructional methods for the delivery of exercise science content to prospective physical educators.

\section{Scope of the Study}

This research project included a pilot study and a modified Delphi investigation to determine the critical exercise science competencies, and associated instructional methods, that are recommended for inclusion in the PETE undergraduate curriculum. The pilot study involved the design of a questionnaire that was later used during a two-round, modified Delphi protocol. The researcher constructed an initial list of theoretical and applied exercise science competencies based on the knowledge, skills, and abilities 
recommended by the American College of Sports Medicine (2000) for individuals working in the field of exercise science. This initial inventory was sent to six expert reviewers in the areas of exercise science (n $=2)$, teacher education $(n=2)$, and physical education teaching $(n=2)$. The reviewers were randomly selected and recruited from the total population of individuals who met the predetermined criteria for Delphi panel selection. The ultimate goal of this component of the research process was to construct the most comprehensive list of theoretical and applied exercise science concepts possible, and to determine the content validity of each questionnaire item. The resultant questionnaire items were restructured into the final Delphi questionnaire in accordance with the expert reviewers' recommendations regarding content and format.

The two-round, modified Delphi procedure that followed involved the repeated circulation of the previously designed questionnaire to a small panel of content experts. Experts in the field of physical education, including (a) exercise science specialists $(\mathrm{n}=10)$, (b) physical education teacher educators $(\mathrm{n}$ $=7)$, and (c) physical education teachers $(\mathrm{n}=3)$, were sampled to determine which theoretical and applied exercise science competencies are critical for the preparation of prospective K-12 physical educators. The Delphi panel members were required to rate each questionnaire item in regards to its theoretical importance and pedagogical relevance. The resulting Likert scale ratings were analyzed and summarized descriptively at the culmination of the first round of questionnaire circulation.

During the second round of the Delphi investigation, the individual and mean group responses from the previous round were shared with the participants. Each panel member was then required to reevaluate his or her position by rating each questionnaire item a second time. Additionally, the panel members were asked to complete an addendum survey concerning their recommendations regarding the four most effective instructional methods for the delivery of exercise science to prospective physical education teachers.

The data collected during the second round of questioning were used to provide a final measure of consensus regarding the critical strength of each exercise science competency. For this study, an item needed to be considered both sufficiently important and pedagogically relevant in order to be considered critical in nature. Each item had to meet the following criteria for consensus (Jacobs, 1996): (a) The item received a mean rating of at least four or higher in the areas of importance and relevance; and (b) the item received at least $75 \%$ of all individual ratings at the four level or higher. Any item that failed to meet these criteria was considered to be non-critical.

\section{Basic Assumptions}

1. The knowledge of certain critical theoretical and applied exercise science competencies is essential for the effective practice of teaching physical education.

2. The Delphi panel members possess some measure of expert understanding regarding the relationship between exercise science theory and application in physical education teaching.

3. The Delphi Method, which typically consists of two to four iterations, provides sufficient opportunity for the generation of a group consensus.

\section{Limitations of the Study}

1. The personal and professional obligations of the panel members often limit the amount of time that each can commit to the decision-making process (Clayton, 1997). 
2. The Delphi Method is designed to achieve a level of consensus among panel members. It is uncertain whether that consensus is achieved through each panel member's thoughtful reconsideration of their position relative to the group, or from the pressure to conform to the group's opinion (Clayton, 1997).

3. The results of the Delphi Method are limited to the specific panel of experts and cannot be generalized beyond the study due to the backgrounds, experiences, and personal biases that influence the decision-making process (Clayton, 1997).

\section{Definition of Terms}

1. Delphi Method - research protocol that provides for the "systematic solicitation and collection of opinions" (Pollard \& Tomlin, 1995, Methodology section, ๆ 3). The Delphi Method "enables researchers to gain consensus from a group of knowledgeable people about a particular topic without the time and expense involved in bringing them together" (Pollard \& Tomlin, 1995, Methodology section, 93 ).

2. Expert - individual who possesses the knowledge and experience to participate in a Delphi investigation (Clayton, 1997). For the purpose of this Delphi study, the panel will consist of physical education practitioners, teacher educators, and exercise science specialists who meet established criterion in the areas of research, teaching, and/or service as related to exercise science and/or school-based physical education.

3. Consensus - gathering of individual evaluations around a mean or median response with minimal variation (Murry \& Hammons, 1995). For the purpose of this Delphi study, a consensus of agreement was achieved when an item obtained a mean rating of four or higher in the areas of importance and relevance with at least $75 \%$ of the participants rating the item a four or higher (Jacobs, 1996).

\section{Significance of the Study}

This study resulted in the determination of a list of fundamental exercise science competencies that physical educators should learn, and the delineation of recommended instructional methods for delivering exercise science content in the PETE curriculum. The limited research in this area demonstrates that the course content and instructional methods associated with traditional exercise science courses may inadequately address the needs of prospective physical educators (Barnett \& Merriman, 1994; Kelley \& Lindsay, 1977, 1980; Miller \& Housner, 1998; Van Donselaar \& Leslie, 1990). Furthermore, inadequate teacher preparation in the foundational subdisciplines (e.g., anatomy, physiology, exercise physiology, biomechanics, and motor development) may contribute to the perceived gap between theory and application that currently confronts the physical education profession. This proposed gap could ultimately manifest itself in the form of ineffective school physical education programs that contribute little to an individual's ability to maintain a physically active lifestyle.

The arguments presented in this chapter have been adopted from my previous scholarly efforts concerning the role of subdisciplinary knowledge in the preparation of physical education teachers (Bulger, Mohr, Carson et al., 2000; Bulger et al., 2001; Bulger, Mohr, Graves, \& Wiegand, 2000; Bulger, Mohr, \& Wiegand, 2003; Townsend, Wiegand, Bulger, Mohr, \& Metzler, 2002). Most notably, Bulger, Mohr, Carson et al. (2000) described the underlying rationale, design, and implementation of a developmental exercise physiology lecture and laboratory course for physical education majors at West Virginia University. This alternative course was implemented to enable PETE students to make more meaningful connections between the involved content and its eventual application in school-based 
settings. Follow-up evaluations indicated that the previously described course design represents a viable alternative to more traditional approaches for delivering exercise physiology content to prospective physical educators within the PETE program (Bulger, Mohr, Graves et al., 2000; Bulger et al., 2003).

As PETE programs continue to experiment with these types of alternative pedagogical approaches to bridge the gap between theory and practice, the need for multi-disciplinary research and enhanced communication becomes readily apparent. This study initiated an important discussion among teacher educators, exercise scientists, and physical educators regarding the critical exercise science competencies and instructional methods that should be incorporated in the PETE curriculum. The results of this scholarly process have hopefully provided a conceptual framework upon which PETE faculty members can make future curricular decisions in the area of exercise science. 


\section{CHAPTER II REVIEW OF LITERATURE}

This review of the literature provides a rationale for this study and is organized into the following three sections: (a) Children's Physical Activity and Health, (b) Bridging the Gap Between Theory and Practice in Physical Education Teacher Education (PETE), and (c) Delphi Method: A Tool for MultiDisciplinary Research and Communication.

\section{Children's Physical Activity and Health}

Children's physical fitness, physical activity, and health are closely related concepts (McKenzie \& Sallis, 1996) that have greatly influenced the curricular orientations of school physical education programs across time. While physical education programs have traditionally advocated the attainment of high levels of physical fitness as an important curricular objective, contemporary scholars have provided a convincing rationale that supports the need for an increased emphasis on the health benefits associated with regular participation in lifelong physical activity (Pate \& Hohn, 1994). There is not a direct relationship between physical activity and physical fitness in children (Pangrazi \& Corbin, 1990, 1993) and other related factors strongly impact a child's fitness performance and trainability (e.g., environment, maturation, and heredity). The current literature indicates that the promotion of children's physical activity has become a more meaningful curricular objective than the attainment of high fitness test scores in school-based physical education programs (Pate \& Hohn, 1994). The maintenance of regular physical activity habits and adequate fitness levels into adulthood is thought to be a more legitimate concern for physical educators than the attainment of high levels of fitness performance.

Accordingly, physical education programs in elementary, middle, and secondary schools currently share the responsibility of promoting regular physical activity as an indispensable component of a healthy lifestyle (National Association for Sport and Physical Education, 2004a). In fact, two of the national content standards for physical education pertain to the student's ability (a) to exhibit a physically active lifestyle, and (b) to achieve and maintain health enhancing levels of physical fitness. Physical educators must facilitate the development of the knowledge, attitudes, and skills that will enable a child to participate in developmentally appropriate levels of physical activity throughout his or her life. McKenzie and Sallis (1996) stated that "while this is not a new goal for physical education, reaching it will require the implementation of both curricular and instructional strategies that are substantially different from those in traditional and sport-oriented programs" (p. 224).

Unfortunately, there is ample evidence to suggest that some school physical education programs have not historically addressed the physical activity or health-related physical fitness needs of all children (Hill \& Randle, 1992; McKenzie, 1999; Ross \& Gilbert, 1985; Ross \& Pate, 1987; U.S. Department of Health and Human Services, 2000). Pate and Hohn (1994) theorized that the physical education profession's failure to positively influence the physical activity habits of children and youth has contributed to decreased public support for school physical education programs despite society's growing appreciation for the importance of regular physical activity and its role in the maintenance of good health. Pate and Hohn argued that "societal support for school physical education has decreased, despite an overall increase in support for activity and fitness programs, because it is society's perception that physical education has not 'delivered the goods.' We suspect that society's collective attitude toward physical education is fundamentally ambivalent" (p. 2). This public ambivalence has recently manifested itself in the form of reduced financial support for school physical education programs, the use of poorly prepared classroom teachers to deliver physical education instruction, and the erosion of state requirements in the subject area of physical education. In order to counteract these disturbing trends, Pate and Hohn suggested that the physical education profession adopt a contemporary mission that promotes "in youngsters adoption of a physically active lifestyle that persists through adulthood" (p. 4). With this 
new focal point or mission statement in mind, it becomes even more imperative that physical educators develop and maintain a complete understanding of the connections between physical activity and health.

\section{The Health and Physical Activity Connection}

Physical inactivity has been formally "recognized as a risk factor for cardiovascular disease (CVD), which accounts for more than half of the adult deaths in the United States each year" (McKenzie \& Sallis, 1996, p. 223). The U.S. Department of Health and Human Services' (1996) historic report on physical activity and health has provided a comprehensive analysis of decades of research. The following major findings were reported by the U.S. Department of Health and Human Services: (a) individuals who are typically sedentary can improve their health by becoming moderately active on a regular basis, (b) physical activity does not need to be strenuous to produce health benefits, and (c) greater health benefits are associated with the increased duration, frequency, and/or intensity of the physical activity.

The physiological and psychological benefits resulting from participation in regular physical activity have been well documented in the literature (American Heart Association, 1996a; Bar-Or, 1999; Biddle, 1995; Bouchard \& Després, 1995; Centers for Disease Control and Prevention \& American College of Sports Medicine, 1995; Corbin \& Pangrazi, 1996b, 1999; Erikssen, 2001; Haskell, 1999; Lee, 1995; Plowman, 1999; U.S. Department of Health and Human Services, 1996; Vuori, 1995). The primary benefits associated with regular physical activity include reduced risk of premature death, cardiovascular disease, diabetes, colon cancer, obesity, orthopedic ailment, depression, and anxiety (Centers for Disease Control and Prevention \& American College of Sports Medicine, 1995; U.S. Department of Health and Human Services, 1996). In addition to the human cost, researchers have projected that:

Physical inactivity and its associated health problems have substantial economic consequences for the U.S. health care system. In the long run, physical inactivity threatens to reverse the decades-long progress that has been made in reducing the morbidity and mortality associated with many chronic conditions such as cardiovascular disease. A physically inactive population is at both medical and financial risk for many chronic diseases and conditions including heart disease, stroke, colon cancer, diabetes, obesity, and osteoporosis. (U.S. Department of Health and Human Services, 2002, p. 4)

Cardiovascular and metabolic health. Bouchard and Després (1995) stated that "regular physical activity has much to offer from a population point of view in terms of risk reduction for overweight and obesity (particularly abdominal obesity), glucose intolerance and non-insulin-dependent diabetes mellitus, blood lipid and lipoprotein abnormalities, hypertension, peripheral vascular disease, cerebrovascular accidents, and coronary heart disease" (p. 273). A number of professional and governmental organizations have authored a variety of position statements summarizing the beneficial effects of a physically active lifestyle in the primary and secondary prevention of cardiovascular, pulmonary, and metabolic disease (American College of Sports Medicine, 1994, 2001; U.S. Department of Health and Human Services, 2001).

Historically, the professional recommendations for exercise prescription have been based on scientific studies that investigated the effects of various types of training protocol on the individual's aerobic performance capacity (Centers for Disease Control and Prevention \& American College of Sports Medicine, 1995). The associated exercise recommendations for healthy adults emphasized the performance of cardiovascular endurance exercise for 20 to 60 minutes at $65 \%$ to $90 \%$ of maximum heart rate or $50 \%$ to $85 \%$ of heart rate reserve on three to five days per week. More recent guidelines indicate that improved cardiovascular health can be achieved for all adults by incorporating at least 30 minutes of moderate intensity physical activity on most, if not all, days of the week (Centers for Disease Control and Prevention \& American College of Sports Medicine, 1995; Haskell, 1999; U.S. Department of Health and 
Human Services, 1996). This 30-minute total can be achieved through the performance of traditional forms of continuous exercise and recreation, such as biking, swimming, walking, jogging, and tennis. The daily requirement of 30 minutes, however, can also be accumulated as the product of brief, intermittent periods of moderate intensity physical activity performed throughout the course of the day, such as gardening, raking leaves, dancing, active play, and walking stairs.

The Centers for Disease Control and Prevention and the American College of Sports Medicine (1995) concluded that "although the earlier exercise recommendations were based on documented improvements in fitness, they probably provide most of the disease prevention benefits associated with an increase in physical activity. However, it now appears that the majority of these health benefits can be gained by performing moderate-intensity physical activities outside of formal exercise programs" (p. 405). This important philosophical transition from an exercise prescriptive model to a lifetime physical activity model for physical activity program design has important ramifications for a wide range of professionals, including physical educators, athletic coaches, exercise scientists, and fitness specialists (Corbin \& Pangrazi, 1994, 1996a; Pangrazi, Corbin, \& Welk, 1996). By decreasing the emphasis on fitness performance and reinforcing the process of becoming physically active on a regular basis, the exercise professional can better address the unique goals and capabilities of each individual (Franks, 1999). For example, the Centers for Disease Control and Prevention and the American College of Sports Medicine (1995) reported that "the health benefits gained from increased physical activity depend on the initial activity level. Sedentary individuals are expected to benefit from increasing their activity to the recommended level. People who are physically active at a level below the standard would also benefit from reaching the recommended level of physical activity. People who already meet the recommendation are also likely to derive some additional health and fitness benefits from becoming more physically active" (p. 404).

Musculoskeletal health. After reviewing the pertinent literature, Vuori (1995) concluded that "physical activity positively influences most structural components of the musculoskeletal system that are related to functional capabilities and the risk of degenerative diseases. Physical activity also has the potential to postpone or prevent prevalent musculoskeletal disorders, such as mechanical low back pain, neck and shoulder pain, and osteoporosis and related fractures" (p. 282). Clinical experience and a growing body of research suggest that individuals who maintain or improve their muscular strength and flexibility through a program of regular physical activity may be less likely to experience low back pain, reduced functional capacity with aging, and various other degenerative musculoskeletal problems (American College of Sports Medicine, 1995, 1998a, 1998b; Centers for Disease Control and Prevention \& American College of Sports Medicine, 1995; National Strength and Conditioning Association, 2001; Plowman, 1999; Pollock \& Vincent, 1999; Shaw \& Snow, 1999; U.S. Department of Health and Human Services, 1996, Vuori, 1995).

In the past decade, the American College of Sports Medicine (1990, 1998b) expanded its recommendations for the quantity and quality of exercise for healthy adults to include a strength or resistance training component. The American College of Sports Medicine recommends that all healthy adults perform a minimum of 1 set of 8 to 10 dynamic strength-training exercises for each of the major muscle groups at least 2 times per week. The participant should employ a resistance that allows for the performance of 8 to 12 repetitions to the point of near fatigue. Older adults and more frail individuals should select a lighter load that allows them to perform 10-15 repetitions per set due to potential orthopedic considerations. These guidelines were later extended to recommend progression models in resistance training for healthy adults who have more advanced muscular fitness goals, such as maximal strength, local muscular endurance, power, and hypertrophy (American College of Sports Medicine, 2002). The American College of Sports Medicine also recommends that flexibility exercises be incorporated into an overall fitness program to develop and maintain joint range-of-motion. Individuals 
should select static and/or dynamic stretches for each of the major muscle groups that can be performed 2-3 days per week (American College of Sports Medicine, 1998b).

Immune system health. While many uncertainties remain and the need for continued scientific investigation is apparent, "an exciting and emerging body of research has suggested that exercise, at least in moderate amounts, can enhance the human immune system. Theoretically, then, this provides a further biological basis for expecting an inverse relationship between physical activity and cancer risk" (Lee, 1995, p. 290). More specifically, an increasing amount of epidemiological research suggests that regular exercise may decrease the risk of some forms of cancer, in particular colon cancer (Lee, 1999).

Furthermore, preliminary data indicates that exercise may also improve a cancer patient's overall quality of life and immune function.

Psychosocial health. Biddle (1995) stated that the contemporary literature in sport and exercise supports a relationship between physical activity and psychosocial health. Biddle reviewed the available research and concluded that exercise positively impacts psychosocial health in a number of areas. Most notably, exercise was found to have moderate-to-large beneficial effects on individuals suffering from depression. Additionally, exercise was shown to positively benefit performance on psychological indices of anxiety, mood, self-esteem, cognitive functioning, and other measures of mental well-being.

Childhood physical activity and adult health. Due to a lack of longitudinal research, there is much less evidence available concerning the benefits of a physically active lifestyle during childhood and adolescence on adult health (Bar-Or, 1999). Bar-Or reported that "no long-term studies exist that support or reject the notion that physical activity during childhood and adolescence is beneficial to adult health. There is, however, some evidence for short-term benefits of enhanced PA [physical activity] during the early years, particularly among children and youth who are at high risk for chronic illness in later years" (p. 165). Casperson, Nixon, and DuRant (1998) provided a comprehensive review of the available epidemiologic research concerning the connection between physical activity and health as applied to children and adolescents. They reported that in many instances, problems exist with the methodological and statistical procedures that have been employed in these epidemiological studies, thus negating what were initially considered to be beneficial results. More recently, Twisk, Kemper, and van Mechelen (2002b) reviewed the findings of six longitudinal studies that investigated the relationship between physical activity and fitness during childhood and risk factors for cardiovascular disease later in life. The studies demonstrated that "physical fitness during youth is associated with a 'healthy' CVD [cardiovascular disease] risk profile later in life, but that body fatness can influence this association. Furthermore, physical activity during youth is not associated with a 'healthy' CVD risk profile later in life. However, the error in measuring physical activity can be partly responsible for this lack of evidence" (p. S49).

Despite this lack of conclusive empirical evidence, Blair, Clark, and Cureton (1989) theorized that there are three means by which increased participation in physical activity during childhood might positively impact adult health: (a) childhood physical activity improves childhood health which then contributes to enhanced adult health, (b) physically active lifestyles during childhood have some type of direct benefit to adult health, and (c) physically active children become physically active adults, who enjoy reduced risk for a variety of chronic degenerative diseases. Unfortunately, "the ideal study to answer the question whether high levels of physical activity and physical fitness during childhood and adolescence lower the risk of developing CVD [cardiovascular disease] later in life is a randomized controlled trial with a lifetime follow-up, in which a large group of children and adolescents is assigned to either a sedentary or an active lifestyle; a study which will probably never take place" for obvious economic, logistical, and ethical reasons (Twisk, Kemper, \& van Mechelen, 2002a, p. S6). 


\section{Professional Recommendations for Children's Physical Activity}

Despite the considerable amount of evidence supporting the relationship between regular physical activity and improved health, many adults continue to lead sedentary lifestyles (U.S. Department of Health and Human Services, 1996). Furthermore, nearly half of American youth 12 to 21 years of age also fail to engage in vigorous physical activity on a regular basis and this participation appears to decrease dramatically during adolescence (U.S. Department of Health and Human Services, 1996). Strand, Scantling, and Johnson (1997) arrived at the following conclusions:

Based on strong evidence, it is safe to say that, in general, children and youth in the United States are neither as active nor as healthy as many believe they should be. It has become increasingly apparent that they are below healthy levels in a number of areas, namely, hypertension, serum cholesterol, and obesity, all of which are risk factors for coronary heart disease and, ultimately, premature death (Gortmaker, Dietz, Sobol, \& Wehler, 1987; Kuntzleman \& Reiff, 1992; Plimpton, 1987; Ross \& Gilbert, 1985; Ross \& Pate, 1987). According to many experts, the problems associated with youth fitness levels are varied; but on the whole, many children and youth are not getting the moderate to vigorous exercise they need to develop, maintain, or improve fitness levels (Durant et al., 1992; Durant et al., 1993; Gilliam, Freedson, Geenen, \& Shahraray, 1981; Janz, Golden, Hansen, \& Mahoney, 1992; MacConnie, Gilliam, Geenen, \& Pels, 1982). (p. 4)

A more recent government report highlights similar concerns that our nation's youth have become increasingly sedentary, unfit, and overweight (U.S. Department of Health and Human Services, 2000). This report to the President, from the Secretary of Health and Human Services and the Secretary of Education, asserts that:

In the long run, this physical inactivity threatens to reverse the decades-long progress we have made in reducing death from cardiovascular diseases and to devastate our national health care budget. In the short run, physical inactivity has contributed to an unprecedented epidemic of childhood obesity that is currently plaguing the United States. The percentage of young people who are overweight has doubled since 1980. (p. 1)

The problems of children's physical inactivity and obesity are complex and multi-factorial in nature (American Heart Association, 1996b; Bar-Or, 2000; Fulton, McGuire, Casperson, \& Dietz, 2001; Sallis, Prochaska, \& Taylor, 2000; Welk, Wood, \& Morss, 2003; Woodfield, Duncan, Al-Nakeeb, \& Jenkins, 2002). Individual (e.g., gender, age, ethnicity, self-efficacy), social (e.g., parental, sibling, and peer support), and environmental (e.g., facility access, time spent outdoors, opportunities to be active) factors have been found to consistently influence the physical activity levels of children and adolescents (Sallis, Prochaska, \& Taylor, 2000). In an effort to reverse this potentially problematic trend regarding children's health, a number of professional organizations have established guidelines for the promotion of children's physical activity and fitness (American Academy of Pediatrics, 1987; American College of Sports Medicine, 1988; American Heart Association, 1996a; National Association for Sport and Physical Education, 1998b, 2004b).

American Academy of Pediatrics. The American Academy of Pediatrics (1987) expressed a number of concerns regarding the future of children's physical fitness and school-based physical education programs. Citing a lack of financial support for school physical education programs and the increasing appeal of various forms of sedentary activity, the American Academy of Pediatrics anticipated a probable decline in children's physical fitness levels. Furthermore, a number of strategies were recommended to encourage youth participation in physical activity: (a) School programs should emphasize the skills needed to participate in a variety of lifetime physical activities, such as cycling, swimming, and tennis; 
(b) schools should decrease the curricular space devoted to more traditional team sports, like basketball, football, softball, and volleyball; (c) schools should provide students with opportunities to engage in fitness-oriented activities that promote lifelong participation in cardiovascular exercise; and (d) pediatricians should assume an active role in advocating school physical education and parental involvement as key components in the development of physically active lifestyles among children and youth.

American College of Sports Medicine. The American College of Sports Medicine (1988) also expressed concern with the status of children's physical fitness and issued an opinion statement that was intended to "provide direction regarding the structure and scope of physical fitness programs for children and youth" (p. 422). The American College of Sports Medicine assumed the position that "physical fitness programs for children and youth should be developed with the primary goal of encouraging the adoption of appropriate lifelong exercise behavior in order to develop and maintain sufficient physical fitness for adequate functional capacity and health enhancement" (p. 422). Among the various specific recommendations for action, the American College of Sports Medicine advocated that: (a) children and youth obtain at least 20-30 minutes of vigorous exercise each day, (b) school programs focus on education and behavior change as a means to encourage engagement in developmentally appropriate physical activity outside of class, (c) parents and health care providers become increasingly involved in the promotion of physical activity by collaborating with school officials and administrators from other community organizations, and (d) physical educators use appropriate strategies and reinforcements for fitness testing.

American Heart Association. The American Heart Association (1996a) has recommended that "persons of all ages should include physical activity in a comprehensive program of health promotion and disease prevention and should increase their habitual physical activity to a level appropriate to their capacities, needs, and interest" (Implementation of Exercise Programs section, ๆ 1). More specifically, the American Heart Association suggested that children must be introduced to the principles of exercise and physical activity at a relatively early age. Schools must provide children with numerous opportunities to learn the skills that will enable them to participate and enjoy physical activity for a lifetime, while being careful not to overemphasize sports and activities that selectively eliminate marginally skilled children. Furthermore, "schools must teach the benefits of exercise and the development and maintenance of exercise and exercise conditioning throughout life" (Schools section, ๆ 1).

International Consensus Conference on Physical Activity. In response to the lack of a scientific consensus regarding "the amount and types of physical activity necessary for health maintenance during the childhood and adolescent years" (Sallis, Patrick, \& Long, 1994, p. 299), the International Consensus Conference on Physical Activity Guidelines for Adolescents was convened (a) to review the literature pertaining to the effects of physical activity on adolescent health, (b) to establish an explicit set of physical activity guidelines for adolescents, and (c) to recommend strategies for the implementation of these guidelines in clinical settings. A group of experts and representatives from a variety of professional, scientific, medical, and governmental organizations who participated in the conference proposed the following basic guidelines:

Guideline 1 - All adolescents should be physically active daily, or nearly every day, as part of play, games, sports, work, transportation, recreation, physical education, or planned exercise, in the context of family, school, and community activities.

Guideline 2 - Adolescents should engage in three or more sessions per week of activities that last 20 min or more at a time and that require moderate to vigorous levels of exertion. (Sallis \& Patrick, 1994, p. 307-308) 
National Strength and Conditioning Association. The National Strength and Conditioning Association (1996) published a formal position statement and literature review pertaining to youth resistance training. The paper focused on "the benefits and concerns associated with regular, moderate intensity youth resistance training programs" (p. 62). The authors reached the following set of conclusions concerning properly designed and supervised youth resistance training programs: (a) resistance training programs are safe for both prepubescent children and adolescents, (b) resistance training programs can lead to significant strength gains in children, (c) resistance training programs can contribute to motor skill and sport performance enhancement in children, (d) resistance training programs can help with injury prevention in this special population, and (e) resistance training programs enhance the psychosocial and overall health of children. A number of other professional associations had previously published policy statements related to the possible benefits and risks associated with youth resistance training (American Academy of Pediatrics, 1990; American Orthopedic Society for Sports Medicine, 1988). These reports also emphasized the importance of developmentally appropriate program design, well-qualified adult supervision, and provision of a safe exercise environment.

National Association for Sport and Physical Education. The National Association for Sport and Physical Education (1998b) established an initial statement of guidelines concerning children's physical activity. The purpose of the paper is to provide a set of appropriate physical activity guidelines for parents and the wide variety of health, education, and recreation professionals who maintain an interest in this area. The National Association for Sport and Physical Education recommended the following physical activity guidelines for elementary children's physical activity:

Guideline 1 - Elementary children should accumulate at least 30 to 60 minutes of age and developmentally appropriate physical activity on all, or most days of the week.

Guideline 2 - An accumulation of more than 60 minutes, and up to several hours per day, of age and developmentally appropriate activities is encouraged for elementary school children.

Guideline 3 - Some of the child's activity each day should be in periods lasting 10 to 15 minutes or more that include moderate to vigorous activity. This activity will typically be intermittent in nature involving alternating moderate to vigorous activity with brief periods of rest and recovery.

Guideline 4 - Extended periods of inactivity are discouraged for children.

Guideline 5 - A variety of physical activities selected from the Physical Activity Pyramid is recommended.

This set of guidelines was recently updated to reflect current concerns with the impending obesity epidemic and the numerous other health issues associated with sedentary living (National Association for Sport and Physical Education, 2004b). The most significant modification to the guidelines concerns the recommendation of a minimum of 60 minutes, and up to several hours of physical activity per day. The revised guidelines continue to reinforce the need for (a) individualized exercise programming, (b) exposure to a variety of forms of physical activity, (c) instruction of self-monitoring skills, and (d) positive reinforcement regarding the process of being physically active, as opposed to the product of becoming more physically fit. The National Association for Sport and Physical Education has also released physical activity guidelines in the interest of helping infants, toddlers, and preschoolers to avoid becoming overweight (National Association for Sport and Physical Education, 2002a).

The National Association for Sport and Physical Education guidelines for children's physical activity are consistent with the recommendations that have previously been presented in the literature (Corbin \& 
Pangrazi, 1994, 1996a; Pangrazi, Corbin, \& Welk, 1996). Pangrazi, Corbin, and Welk (1996) proposed that children should accumulate 30 to 60 minutes of moderate-intensity activity throughout each day during sporadic bouts of active play. Additionally, the importance of lifestyle activities, such as walking to school or performing household chores, should be emphasized as valuable contributors to good health. Personalized workloads, opportunities for motor skill acquisition, exposure to a variety of physical activities, and a focus on behavior change are also considered to be important characteristics of an effective physical activity program.

While there is obvious merit to the development of these types of guidelines for promoting children's physical activity and health, critics have pointed out the fact that "there is only marginal evidence that physical activity is beneficial for health during childhood and adolescence, and that an improvement of physical activity during childhood will last forever" (Twisk, 2001, 617-627). Furthermore, a clear doseresponse relationship concerning the frequency, intensity, time, and type of physical activity required to reduce the risk for chronic degenerative disease has not yet been established for children due to a lack of true experimental research. Accordingly, the current physical activity guidelines represent an estimate based on field experience and the best available scientific evidence.

\section{Physical Activity in School Physical Education Programs}

The disturbing findings regarding decreased physical activity levels among adolescents (U.S. Department of Health and Human Services, 1996) and the proposed link between childhood inactivity and sedentary living in adulthood, interact to provide a justification for the promotion of physically active lifestyles among children and adolescents in school physical education programs (National Association for Sport and Physical Education, 1998b, 2004b). McKenzie and Sallis (1996) suggested that "engaging children and adolescents in physical activity and teaching them behavioral skills related to developing and maintaining appropriate physical activity could help prevent future generations of adults from becoming so sedentary" (p. 224).

Despite (a) the contentions that children and youth are not as physically active as is recommended for the maintenance of good health, (b) the belief that school physical education programs can play a vital role in facilitating the development of physically active lifestyles, and (c) the demonstrated effectiveness of certain curricular interventions for the promotion of physical activity (Strand, Scantling, \& Johnson, 1997), many school physical education programs may not address the physical activity needs of all students (Hill \& Randle, 1992; McKenzie, 1999; McKenzie \& Sallis, 1996; National Association for Sport and Physical Education, 1998c; Pate \& Hohn, 1994; Ross \& Gilbert, 1985; Ross \& Pate, 1987).

Accordingly, a number of professional and governmental agencies have also acknowledged the potential importance of school physical education programs in the advocacy of physical activity (American Academy of Pediatrics, 1987; American College of Sports Medicine, 1988; American Heart Association, 1996a; National Association for Sport and Physical Education, 1998b, 2004b; Sallis \& Patrick, 1994; Sallis, Patrick, \& Long, 1994; U.S. Department of Health and Human Services, 1996). McKenzie (1999) reported that schools, as an established societal infrastructure, "serve nearly all children and adolescents, have a staff of physical activity experts, require almost all children to take some form of physical education, and house facilities and equipment specifically designed to promote physical activity" (p. 16).

The U.S. Department of Health and Human Services (1997) developed a set of physical activity promotion guidelines for school and community programs after reviewing the literature related to the consequences of physical inactivity. The key principles underlying these guidelines state that physical activity programs for young people are most effective when they (a) emphasize participation in enjoyable activities that can easily be performed throughout life, (b) offer the opportunity to engage in a variety of 
age and developmentally appropriate noncompetitive and competitive activities, (c) provide young people with the skills and confidence to participate in regular physical activity, and (d) promote physical activity from a multi-disciplinary perspective that integrates various resources of school and community programs. The related recommendations for schools and communities directly address the following potential areas of concern in children's physical activity promotion:

1. Establishing policies that promote participation in enjoyable, lifelong physical activity.

2. Providing appropriate, well-supervised physical and social environments.

3. Implementing sequential physical education curricula and instruction in grades K-12.

4. Implementing sequential health education curricula and instruction in grades K-12.

5. Providing opportunities for participation in extracurricular physical activity programs.

6. Encouraging family involvement in physical activity promotion efforts.

7. Providing training for school staff, teachers, coaches and other community personnel.

8. Utilizing available health services for the assessment of physical activity levels.

9. Providing developmentally appropriate community sports and recreation programs.

10. Evaluating the quality of physical activity instruction, programs, and facilities.

A more recent report from the U.S. Department of Health and Human Services (2000), in cooperation with the Department of Education, offered additional strategies for promoting better physical activity among young people. The report acknowledged the important role potentially played by families, schools, after-school care programs, and youth sports and recreational programs in the area of physical activity promotion. The following ten specific strategies were recommended:

1. Educate parents and guardians about the importance of children's physical activity.

2. Provide all children with quality, daily physical education.

3. Publish and disseminate instructional resources for use in physical education and other programs.

4. Facilitate greater collaboration between state education and health departments.

5. Enable more after-school programs to provide additional physical activity opportunities.

6. Provide more young people with access to community recreation and sports programs.

7. Educate community recreation and sports program leaders about age-appropriate physical activity.

8. Support environmental approaches to enhance physical activity (e.g., bike paths, parks, trails).

9. Maintain a continual media campaign promoting regular participation in physical activity.

10. Monitor youth physical activity, fitness, and program accessibility in each state.

The similarities between these two sets of guidelines are readily apparent and highlight the need for comprehensive approaches to physical activity promotion that address a wide range of social, individual, and environmental constraints that could possibly influence the physical activity participation of children and adolescents. There are no simple answers to the highly complex issues of children's physical inactivity and obesity, but it seems relatively clear that any viable solution will involve collaboration among a number of constituencies within each community (e.g., elected officials, school administrators, teachers, health care professionals, day care operators, parents and guardians, community recreation personnel, youth program volunteers). While this degree of collaboration is often difficult to obtain, effective school-based interventions for physical activity promotion have been described in the literature (Casperson, Nixon, \& Durant, 1998; McKenzie \& Sallis, 1996). Casperson, Nixon, and Durant (1998) reported that "although the number of studies that have examined the effectiveness of interventions to promote increased physical activity and exercise among children and adolescents is limited, they nonetheless suggest that properly designed interventions can be effective in this population" (p. 388).

Child and Adolescent Trial for Cardiovascular Health (CATCH). The CATCH intervention was a school-based health promotion program, sponsored by the National Heart, Lung, and Blood Institute that "targets physical activity, eating, and smoking behavior and includes classroom curricula, school 
environment changes involving physical education and food service, and family involvement components" (McKenzie \& Sallis, 1996, p. 231). This large-scale intervention included the implementation of the CATCH program in 96 elementary schools across a 3-year period (Perry et al., 1990). The major findings indicated that school-based interventions can be effective in (a) reducing children's dietary fat intake and increasing their self-reported levels of physical activity, (b) modifying the school environment to promote healthier food choices, and (c) increasing amounts of moderate to vigorous physical activity during physical education classes (McKenzie et al., 1996; Perry \& Sellers, 1997; Webber et al., 1996). Furthermore, a follow-up study suggested that the dietary and physical activity behavioral changes initiated during the elementary school years as a result of the CATCH intervention were maintained into early adolescence (Nader et al., 1999). The CATCH research methodology also contributed a number of important advances to the assessment strategies for monitoring children's physical activity participation within school-based physical education classes (McKenzie \& Strikmiller, 1994).

Heart Smart Program. The Heart Smart Family Health Promotion Program is described as "a multidisciplinary, school-based program for cardiovascular risk reduction among high-risk children and their families" (Johnson et al., 1991, p. 1305). This elementary school-based health intervention emphasized the importance of a number of key behavioral issues including proper nutrition, regular physical activity, and smoking/drug prevention (McKenzie \& Sallis, 1996). The primary research findings demonstrated that both children with elevated risk factors for cardiovascular disease and their parents experienced a number of significant benefits as a result of their participation in the Heart Smart program (Johnson et al., 1991). The participating parents and children "showed positive changes in eating habits and physical activity and significant changes in knowledge and blood pressure levels, while the children halted their weight gain" (Johnson et al., 1991, p. 1306).

Sports, Play, and Active Recreation for Kids (SPARK). This intervention focuses exclusively on the promotion of elementary school-aged children's participation in regular physical activity (McKenzie \& Sallis, 1996). The SPARK program includes a comprehensive physical education curriculum and the accompanying instructional materials for weekly self-management workshops during which the children plan and assume responsibility for their outside-of-class physical activity. Sallis and McKenzie (1997) investigated the effects of the SPARK curricular intervention on the physical activity patterns of fourth and fifth grade children. Results suggested that a health-related physical education curriculum provided children with a significantly greater amount of physical activity than the control condition. The SPARK intervention did not, however, positively influence student engagement in out-of-class physical activity. A number of additional studies have supported the effects of SPARK on the quality of physical education lessons in elementary schools (McKenzie \& Alcaraz, 1994; McKenzie, Sallis, Faucette, \& Kolody, 1997) and the resulting impact of health-related physical education on student academic achievement (Sallis et al., 1999).

\section{Implications for PETE Programs}

PETE programs collectively share the responsibility of providing prospective teachers with the subject matter knowledge, pedagogical knowledge, and pedagogical content knowledge (Shulman, 1987) needed to effectively foster the development of the psychomotor, cognitive, and affective characteristics that will enable a child to enjoy a healthy, physically active lifestyle into adulthood. The growing national concern with physical activity and its role in the maintenance of good health (U.S. Department of Health and Human Services, 1996) should be used to help determine the course content and instructional methodologies employed within the PETE curriculum. This appreciation for regular physical activity and its accompanying health-related benefits exemplifies the need for physical educators who are adequately prepared to instruct and apply the concepts which may enhance a child's appreciation for regular physical activity as an important component of a healthy lifestyle. While guidelines for the promotion of physical 
activity in the schools have been established and a number of well-conceived school-based interventions are empirically supported in the literature, the preparation of prospective physical educators in this area represents a significant concern.

It seems relatively clear that a number of critical theoretical and applied exercise science concepts certainly contribute to a physical educator's instructional effectiveness regarding the promotion of physical activity, but there is some evidence to suggest that PETE programs may inadequately prepare prospective physical educators to instruct and apply these important concepts in the promotion of physically active lifestyles. If the physical education profession is to play a more meaningful role in the fight against children's physical inactivity and the associated chronic degenerative diseases that typically manifest themselves later in adulthood, PETE professionals need to become more adapt at preparing prospective physical educators to actively engage in multi-disciplinary approaches to physical activity promotion. While school physical education programs will never represent a stand alone solution to the current obesity epidemic, it remains a potentially significant piece of the puzzle.

\section{Bridging the Gap between Theory and Practice in PETE}

The integration of theory into practice remains a problematic issue in many PETE programs (Corbin, 1993; Feingold, 1994; Lidstone \& Feingold, 1991; Metzler, 1994). This area of concern emanates directly from the foundational subdisciplines of physical education. The subdisciplines are considered to be the academic content areas from which the undergraduate physical education curriculum derives its foundational knowledge and conceptual framework (Estes, 1994). Foundational subdisciplinary courses in the PETE curriculum often include biomechanics, anatomy, physiology, motor learning, history/philosophy of movement, and sociocultural factors of movement (Bahneman, 1996; Estes, 1994). Over the past half century, physical education has evolved from a profession primarily concerned with the instruction of exercise, games, and sport to a collection of disjointed subdisciplines and loosely related professions (Corbin, 1993, 1994). The benefits and liabilities associated with this philosophical transformation have greatly impacted the current status of many PETE programs.

\section{Historical Perspectives}

Prior to the 1950's, PETE programs focused on the professional development of the technical skills and knowledge required for the teaching and coaching of sport (Corbin, 1993, 1994). While this professional approach to the preparation of prospective physical educators seems to make intuitive sense, critics argued that the lack of an organized body of knowledge illustrated physical education's inadequacy as an academic discipline within the higher education community (Henry, 1964).

During the 1960's and 1970's, PETE programs embraced a curricular orientation that placed a greater emphasis on subdisciplinary knowledge (Corbin, 1993, 1994). New courses in subdisciplinary areas like exercise physiology, sport psychology, biomechanics, and motor learning were incorporated into PETE curricula throughout the country. This curricular shift occurred as physical education departments, schools, and colleges sought to establish their academic legitimacy (Henry, 1964), and identify a theoretical basis for the determination of appropriate professional practices (Morford, 1972; Siedentop, 1972).

The progressive movement towards a subdisciplinary approach contributed greatly to physical education's evolution into a credible field of study within higher education. Accordingly, the newly established research programs at colleges and universities conducted numerous scientific investigations that lead directly to (a) a considerable expansion of the existent subdisciplinary knowledge base in physical education, (b) the wider acceptance of physical education as a viable area of study within higher 
education, and (c) the improved standing of physical education within the academic community (Corbin, 1993, 1994; Ross, 1981).

The disciplinary approach to PETE was firmly established at most major universities by the 1980 's, but departments continued to struggle with a number of issues including "legislative directives, university acceptance, attracting students, attracting grants, and a change in departmental focus" (Corbin, 1993, p. 549). Lidstone and Feingold (1991) stated that the literature of the period accurately reflects the dramatic knowledge growth and scholarly debate that characterized physical education as "a discipline/profession in transition, struggling for identity, arguing about who they were and what they should be about" (p. 241). The following debates raged as scholars attempted to make sense of physical education's rapid evolution:

Discipline versus profession, basic versus applied, quantitative versus qualitative, theory versus practice, reductionism versus chaos, specialization and fragmentation versus integration and collaboration, physical education versus kinesiology versus physical activity, Ziegler (1983) versus Shepard (1983), Hoffman (1985) versus Thomas (1985), Greendorfer (1987a) versus Hoffman (1985) and Thomas (1985, 1987), Newell (1990a, 1990b, 1990c) versus the world. (Lidstone \& Feingold, 1991, p. 241)

PETE professionals continue to struggle with a number of these issues concerning the integration and application of subdisciplinary knowledge in the practice of teaching physical education. This subdisciplinary orientation has undoubtedly contributed to physical education's evolution into a respected and valued member of the university community (Corbin, 1993, 1994). Conversely, the institutional and societal contexts that shaped this evolutionary process have also contributed to physical education's current state of overspecialization and fragmentation (Lawson, 1991). Corbin (1993) theorized that the disciplinary movement has ultimately resulted in the abandonment of PETE undergraduate and doctoral programs at numerous universities and the decreased emphasis on the provision of social services for the clients, learners, and patients whom physical education professionals have historically instructed.

\section{The Gap between Theory and Practice}

The development of the various subdisciplines was initially intended to improve the instructional effectiveness of physical educators by making connections with other established academic disciplines (O'Hanlon \& Wandzilak, 1980). There is little evidence to suggest that the proliferation of research in the foundational components of physical education has significantly influenced the instructional practices of in-service physical educators, however (Bain, 1981; Corbin, 1994; Locke, 1990; Ross, 1981; Siedentop \& Locke, 1997). Locke (1990) argued that teacher educators have mistakenly accepted the notion that effective instruction in physical education is dependent on subdisciplinary knowledge with little debate, reflection, or evidence.

Lawson (1990) theorized that when physical education programs aligned themselves with the subdisciplines they began to neglect many unique features of the physical education profession and distance themselves from an instructional orientation thus furthering the gap between theory and practice. As the search for academic credibility as sport scientists became increasingly important, the commitment to the preparation of students for professional careers as physical educators has been neglected (Livingston, 1996). Similarly, Shea (1985) argued that the "fragmentation of the discipline isolates each of the subdisciplines from each other and all of them from the totality of physical education" (p. 46).

Ross (1981) proposed that the primary variable accounting for the widening gap between theory and practice is the assumption that undergraduate physical education students possess the ability to integrate theoretical knowledge into professional practice on their own, without deliberate assistance from the 
PETE faculty. PETE programs typically include courses based on subdisciplinary knowledge (Bahneman, 1996), but fail to integrate the pedagogical concepts that will enable the practitioner to successfully apply this information in a physical education setting (Bain \& Poindexter, 1981; Loughery, 1985; Robertson \& Heyden, 1985).

The expectation of a successful leap from theory to practice may become even more unreasonable as undergraduates are repeatedly placed in less than ideal instructional environments (Bain \& Poindexter, 1981; Collier \& O'Sullivan, 1997; Korthagen \& Kessels, 1999; Siedentop \& Locke, 1997). These questionable environments provide future physical educators with inappropriate practice opportunities for the application of complex kinesiological, physiological, and pedagogical concepts.

In most PETE programs, introductory courses in professional preparation continue to emphasize the delivery of foundational knowledge in lecture and discussion formats which may deter the student's active engagement in the learning process (Collier \& O'Sullivan, 1997). Locke (1990) asserted that the "traditional model for the foundation course - 50-minute lectures, multiple-choice exams, pursuit-rotor laboratory projects, and reading assignments that are instruments of torture - certainly does not appear to have been devised to influence teacher behavior" (p. 138). Traditional instructional methods in teacher education programs typically reinforce the passive routines of students rather immersing them in the types of engaging activities that foster the development of rich, flexible, and expandable knowledge structures (Tinning, 1988). Housner and French (1994) also cautioned against the continued use of traditional instructional methods within the PETE curriculum:

We must be careful not to hypocritically espouse the importance of educational practices such as active learning, curricular relevance, and authentic assessment and then place students in large lecture classes in which knowledge is transmitted passively and assessed only through the use of objective tests. (p. 243)

There is evidence to suggest that students are generally not afforded opportunities to apply theoretical knowledge from their previous educational experiences until concluding field placements and internships (Bahneman, 1996; Collier \& O'Sullivan, 1997). Furthermore, Siedentop and Locke (1997) theorized that the failure of PETE programs to positively influence the quality of school physical education was directly linked to the repeated use of disconnected classroom experiences and discontinuous field placements within teacher preparation.

Inadequate educational opportunities and passive exposure to the PETE curriculum compound the difficulties associated with transforming theory into practice. Collectively, these instructional inadequacies may hinder the development of the prospective physical educator's pedagogical content knowledge (Griffin, Dodds, \& Rovegno, 1996). Pedagogical content knowledge is defined as the content and pedagogical knowledge unique to teachers in a particular specialty area (Housner, 1991; Rickard \& Boswell, 1991; Shulman, 1987). This knowledge includes an understanding of the instructional methods and/or techniques for effectively facilitating student learning.

Pedagogical content knowledge deficiencies may be directly linked to an acute lack of both subject matter knowledge (Siedentop \& Eldar, 1989) and pedagogical knowledge (Housner, 1991; Shulman, 1987), i.e. management routines, organizational strategies, and activity structures. Evidence suggests that inadequacies in pedagogical content knowledge are associated with a teacher's inability to use, integrate, and/or transform theory into practice (Housner, 1991; Rickard \& Boswell, 1991; Rovegno, 1993). Accordingly, the theoretical subject matter knowledge that prospective physical educators obtain from their subdisciplinary coursework may be of little practical value if the instructors fail to employ specific educational interventions that are designed to develop the students' related pedagogical content knowledge (Rickard \& Boswell, 1991). 


\section{The Gap between Theory and Practice in Exercise Science}

Exercise science is generally considered a critical subdisciplinary component in the preparation of physical education teachers (National Association for Sport and Physical Education, 1998a). The following outcomes from the National Association for Sport and Physical Education guidelines for PETE programs relate explicitly to the role of exercise science content knowledge in the preparation of prospective physical educators:

1.3 Describe and demonstrate concepts and strategies related to skillful movement and physical activity (e.g., F.I.T.T. principle, offense/defense, distributed practice). (p. 5)

1.4 Describe and apply disciplinary knowledge (concepts and principles to skillful movement, physical activity, and fitness). (p. 6)

1.6 Analyze current physical activity issues based on historical, philosophical, sociological, and psychological perspectives. (p. 6)

1.7 Describe the organic, skeletal, and neuromuscular structures of the human body, identify how these systems adapt to skillful movement, physical activity, and fitness, and analyze their contributions to motor performance. (p. 6)

The theoretical and applied exercise science concepts acquired within the PETE curriculum should provide prospective teachers with the knowledge and skills needed to effectively facilitate the development of the physical, cognitive, and affective characteristics that will enable a child to enjoy a healthy, physically active lifestyle. Philosophical questions concerning the borderline between the fields of exercise science and physical education teaching have proven to be quite problematic, however. PETE programs have frequently struggled with a number of issues regarding the theoretical area of exercise science and its applicability to physical education (Barnett \& Merriman, 1994; Karper, 1997; Miller \& Housner, 1998; Van Donselaar \& Leslie, 1990). The course content and instructional methods represent two areas of concern within the PETE curriculum.

Course content. While courses in exercise science and fitness have been previously identified as the undergraduate physical education major's primary source of knowledge (Mood, 1971), questions regarding the relevancy of the associated course content remain. Kelley and Lindsay (1977) investigated the knowledge obsolescence of practicing high school physical educators in the domains of adaptive physical education, athletic training and conditioning, curriculum, exercise physiology, and instructional methodology. The results indicated that approximately $89 \%$ of the participants failed to achieve the established criteria for being current in the domain of exercise physiology. In a follow-up study, Kelley and Lindsay (1980) employed a similar research methodology to compare the content knowledge of preservice and in-service physical educators. The pre-service physical educators demonstrated a relatively high degree of knowledge obsolescence in exercise physiology. A criterion score of 34 was designated as the minimum level of competency and the mean score for the pre-service student group was 26. Although limited, these findings (Kelly \& Lindsay, 1977, 1980) suggested that research developments in exercise physiology at that time were not being used to update the related curricular content in PETE programs.

More recently, Barnett and Merriman (1994) compared the health-related physical fitness knowledge of physical education and non-physical education majors using a 27 question multiple choice examination at a single university. Barnett and Merriman reported that the majors scored significantly higher than the non-majors on the exam, $76 \%$ and $60 \%$ respectively. With the exception of the freshman group, results indicated that the physical education majors out-performed the non-physical education majors across each grade level as well. The physical education majors also demonstrated an incremental increase in physical 
fitness knowledge across grade level that was not evident in the control group. Barnett and Merriman concluded that this "progressive increase across grades in knowledge of physical fitness among physical education majors" indicated that PETE programs do increase a prospective physical educator's knowledge of physical fitness (Discussion section, $\mathbb{~} 1$ ). While these findings are definitely positive, Barnett and Merriman questioned why this apparent increase in fitness knowledge has not translated to improved fitness programming in the schools.

Miller and Housner (1998) extended this line of research "to assess the health-related physical fitness knowledge of pre-service and in-service physical educators and graduate students in physical education and exercise physiology" (Abstract section, \ 1). Miller and Housner administered a 40-item multiple choice examination that assessed knowledge in the five components of health-related fitness. The findings suggested that the pre-service and in-service physical educators who participated in this study possessed an inadequate knowledge of health-related physical fitness. With the exception of graduate students in exercise physiology and physical education, who scored $83.13 \%$ and $71.75 \%$ respectively, participants in the study scored below $70 \%$ on the test. An increasing trend in the scores of the pre-service physical educators provided support for the findings of Barnett and Merriman (1994). However, the pre-service physical educators' test scores remained below 70\%. Miller and Housner (1998) concluded that the poor performance of the pre-service group may be related to a number of factors including: (a) the educational background of the participants prior to enrollment in the PETE program, (b) the nature of the PETE curriculum, and (c) the lack of adequate educational experiences in the area of health-related physical fitness. Miller and Housner suggested that PETE programs need to evaluate the content of exercise physiology courses for pre-service physical educators in terms of content validity and pedagogical relevance.

Although limited, the previously described studies (Barnett \& Merriman, 1994; Kelley \& Lindsay, 1977, 1980; Miller \& Housner, 1998) may be indicative of several problems associated with the relevancy of the exercise science content typically taught to physical education majors. Locke (1990) proposed that practicing physical educators fail to apply subdisciplinary knowledge because it is basically irrelevant to their work in school settings. It is entirely possible that "the model of the rational teacher who bases instructional decisions on research-based knowledge from the foundational sciences does not describe how most teaching gets done in physical education classes" (Locke, 1990, p. 137). Corbin (1994) also contended that subdisciplinary knowledge is of little practical value if practitioners are unable to effectively integrate it into the teaching of physical education. Research in the subdisciplines of physical education appears to have fragmented and intellectualized the field while neglecting the more practical issue of application (Greendorfer, 1987b; Hellison, 1991).

Siedentop (1972) alluded to this developing problem concerning the relevance of subdisciplinary knowledge to the practice of teaching physical education when he described the following characteristics of the typical physical education major's plan of study:

He will have had from 6 to 9 hours in the biological sciences and another 3 to 5 hours in exercise physiology. He might understand the energy sources contributing to cardiovascular functioning during exercise stress, but he might not be able to set up intelligent exercise programs for various age groups in the community he serves. He probably is better prepared than his predecessors to enter graduate school where disciplinary study is solidly entrenched, but he is not well prepared to effect significant behavior change as a teacher in the schools. (p. 96)

Similarly, McKenzie and Sallis (1996) maintained that "the trend to require potential physical education specialists to take more theoretical and scientific courses while reducing their exposure to instructional methods, program development and assessment, and physical activities is not necessarily 
positive" (p. 237). Physical educators who graduate from these theoretically oriented courses of study may be "better prepared to teach sedentary, cognitive-focused classes than they are to teach students to be physically active" (McKenzie \& Sallis, 1996, p. 237-238).

Increased scientific interest concerning the role of biological maturation in the determination of physiological responses to exercise has lead to a dramatic expansion of knowledge in the field of developmental exercise physiology (Rowland, 1996). While our current understanding of developmental exercise physiology remains incomplete, it seems clear that a child's physiological response to exercise and his or her physical activity needs differ markedly from those of an adult. Practicing physical educators must maintain an adequate understanding of these developmental differences if they are to effectively manage the ethical, performance, and safety issues that could potentially arise in children's physical activity settings.

In light of these developmental differences, Karper (1997) questioned why PETE programs continue to educate prospective physical education teachers in a subdiscipline that emphasizes adult responses to physical activity. Karper argued that it is unnecessary for a physical education major to develop a knowledge structure that is representative of the usual material covered in traditional exercise physiology courses. For example, Karper stated that educational materials concerning resistance training, cardiovascular dynamics, graded exercise testing, and exercise prescription have limited significance or applied value for the practicing physical educator unless they are specifically related to children. PETE programs must recognize the potential significance of the expanding knowledge base in developmental exercise physiology by initiating curricular revisions that increase the relevancy of the course content for the practicing physical educator.

Instructional methods. The instructional methods associated with exercise science courses may also contribute to the gap between theory and practice. Barnett and Merriman (1994) suggested that "perhaps teacher preparation programs have not provided sufficient integration between the learning of physical fitness concepts in element one and the application of these concepts in element three" and that further research in this area is definitely needed (Discussion section, $\uparrow 2$ ). If students are not provided with instructional methodologies that are specifically designed to relate theory to practical problems, they typically regard the theory as being irrelevant and educational practice remains largely unaffected by the continually expanding body of subdisciplinary knowledge (Bain \& Poindexter, 1981).

Dodds (as cited in Van Donselaar \& Leslie, 1990) reported that introductory exercise physiology course enrollment traditionally includes students from a variety of majors who have different intentions for the application of the course content in their future professions. Course enrollment typically includes students who are majoring in physical education, athletic training, exercise physiology, athletic coaching, physical therapy, and so forth. It is highly unlikely that the unique educational needs of each student can be satisfactorily addressed in a course with this type of diverse enrollment (Van Donselaar \& Leslie, 1990).

To further compound this problem, the introductory anatomy and physiology courses required in many PETE programs are likely to be (a) taught outside of the PETE program, (b) instructed by nonteacher educators, and/or (c) influenced little by PETE faculty regarding course content and instructional methods (Verner, 1991). While subdisciplinary specialists maintain a more complete understanding of the involved subject matter, they may lack the knowledge and ability to integrate the essential pedagogical concepts that make the theory to practice transition a workable proposition. Bain and Poindexter (1981) suggested that specialists in the subdisciplines are often unwilling to dilute their theoretical course content with issues of practical application. This reluctance to directly address theory and application may be reinforced by "the discipline-based faculty member's inexperience in public school teaching, and in teaching sports as well" (Bain \& Poindexter, 1981, p. 40). The diverse nature of the course enrollment, 
associated time constraints, and instructor characteristics may interact to produce an educational environment that does not readily facilitate the translation of theory into the practice of teaching physical education.

Proposed solutions for bridging the gap. As previously discussed, bridging the gap between subdisciplinary theory and practical application remains a challenging task for students and instructors (Robertson \& Heyden, 1985). The course content and instructional methods associated with traditional exercise science courses represent two particular areas of concern within PETE programs. A variety of methods have been suggested for countering these problems and narrowing the gap between theory and practice. In order for this bridging process to occur, it is generally accepted that the course content, instructional methods and roles of both the student and instructor must be altered within PETE programs (Collier \& O'Sullivan, 1997). Furthermore, a balance should be established between competing philosophical perspectives within the specific subdisciplines. Knudson (2003), for example, recently stated the need for a "careful balance of biological and mechanical foundations learned in the context of application to real-world human movement problems" within introductory biomechanics courses (The Need for Balance section, $\uparrow 1$ ).

PETE programs must employ instructional methods that foster the development of pedagogical content knowledge and enable students to effectively utilize subdisciplinary knowledge when resolving practical educational problems (Bain \& Poindexter, 1981). The learner must become a more active participant in the teaching-learning process, while the teacher educator must bear the increased responsibility of disseminating current theoretical information in combination with accepted pedagogical practices (Loughery, 1985). Problems-based approaches to teaching exercise physiology, for example, afford students unique opportunities for critical thinking, cooperative learning, independent decisionmaking, and lifelong learning (Dollman, 2003).

The use of curricular strands has been recommended as one possible solution to the theory to practice dilemma in teacher education (Bulger, Mohr, Carson, et al., 2000; Bulger et al., 2001; Feingold, 1994; Livingston, 1996; Miller \& Housner, 1998; Thomas \& Thomas, 1989). Curricular strands are those critical themes or perspectives typically introduced during the foundational semesters of the undergraduate curriculum and systematically revisited in a variety of instructional contexts. The continual and progressive nature of this reinforcement conceptually fosters the development of a more complex and highly integrated knowledge structure concerning the instruction of physical education. For example, Bulger, Mohr, Carson, et al. (2000) advocated the use of the newly revised Physical Best materials (American Association for Health, Physical Education, Recreation and Dance, 1999) for the infusion of a health-related physical fitness education strand into the PETE curriculum.

Other proposed solutions in the subdiscipline of exercise science have included (a) the development of professional committees comprised of subdisciplinary experts (Ross, 1981), (b) the utilization of the Basic Stuff Series (Kneer, 1981) in teacher education (Bain, 1981; Loughery, 1985), (c) the implementation of culminating experiences that are designed to enhance the integration of subdisciplinary knowledge (Robertson \& Heyden, 1985), (d) the increased emphasis on developmental exercise physiology concepts (Bulger, Mohr, Carson, et al. 2000; Karper, 1997; Miller \& Housner, 1998), and (e) the continued evaluation of instructor qualifications and course content (Bulger, Mohr, Carson, et al., 2000; Miller \& Housner, 1998; Verner, 1991). Furthermore, Dodds (as cited in Van Donselaar \& Leslie, 1990) recommended the use of separate or team-taught exercise physiology labs for physical education majors.

Bain and Poindexter (1981) suggested emphasizing the application of pedagogical concepts within subdisciplinary courses, integrating subdisciplinary concepts into professional development courses, and developing new courses which focus specifically on bridging the gap between theory and practice. For 
example, Bulger, Mohr, Carson, et al. (2000) provided a detailed description of a developmental exercise physiology course for prospective physical educators that was specifically designed "to facilitate students' ability to bridge the existent gap between theory and practice in the area of exercise physiology" through the infusion of subject matter knowledge, pedagogical knowledge, and pedagogical content knowledge into a single course (p. 166). The described course involved the dissemination of pedagogically relevant exercise physiology concepts in both lecture and applied laboratory instructional settings that were developed for prospective physical education teachers. Although limited, course assessment data indicated that this type of innovative course (Bulger, Mohr, Carson, et al.) may represent a viable alternative to more traditional exercise physiology courses in terms of student subject matter knowledge and pedagogical content knowledge acquisition (Bulger, Mohr, Graves, \& Wiegand, 2000; Bulger, Mohr, \& Wiegand, 2003). Ultimately, however, longitudinal data concerning student knowledge retention and instructional behavior must be collected over time to determine the true effectiveness of the various components of the PETE curriculum (Bulger, Mohr, Carson, et al., 2000; Miller \& Housner, 1998; Mohr, Bulger, Graves, \& Wiegand, 1999).

Of particular interest, Van Donselaar and Leslie (1990) conducted a survey of PETE programs to investigate the current and recommended practices for preparing physical education teachers in exercise physiology. Results illustrated that the gap between exercise physiology theory and practice in physical education remains a problem. The findings supported the need for "increased attention to physical education teaching applications, reduced reliance on student initiative in making such applications, improved preparation for physical education majors to teach physical fitness concepts, and more collaboration between EP [exercise physiology] and TE [teacher education] personnel" (Van Donselaar \& Leslie, 1990, p. 215).

When appropriately implemented, the previously mentioned solutions may enhance the development of the prospective physical education teacher's pedagogical content knowledge in relation to exercise science. Specific strategies have been used and show great promise in facilitating knowledge growth and cognitive processing skill in novice teachers (Housner, 1991). These educational interventions require students to become more actively involved in the teaching-learning process, while requiring the instructor to disseminate relevant theoretical knowledge into sound pedagogical practice. Specific examples of these interventions include the use of cognitive mapping techniques (Morine-Dershimer, 1989), case studies (Collier \& O'Sullivan, 1997; Shulman, 1986; Wilson \& Williams, 2001), observations of effective instruction, and early field experiences in pedagogically focused environments (Housner, 1991). Furthermore, reflective and problem-based activities also merit attention (Fernandez-Balboa, Barrett, Solomon, \& Silverman, 1996). Estes (1994) advocated the study of epistemology in PETE as a curricular framework and common language by which the prospective physical educator could develop a more complex understanding of subdisciplinary knowledge.

The restructuring of the foundational component of the PETE curriculum and assessment of the effectiveness of these alternative curricular models are complex, ambitious endeavors. In order to meet this challenge, there must be increased collaboration between physical education teacher educators and experts in the foundational subdisciplines. This enhanced communication could potentially facilitate the design, implementation, and assessment of foundational subdisciplinary courses that effectively narrow the gap between theory and practice. Van Donselaar and Leslie (1990) reported that this type of collaboration is unlikely and that dissemination of exercise physiology theory would continue to rest with exercise physiology specialists who may lack the knowledge and ability to relate this material to the practice of teaching physical education.

The undergraduate PETE curriculum is an area in need of continual evaluation and refinement (Bahneman, 1996). The clarification of educational philosophies, the development of new curricular perspectives, and the initiation of meaningful curricular change are difficult processes that could 
undoubtedly benefit from systematic evaluation. Educational researchers must expend the time and effort to design, implement, and assess effective strategies for teacher preparation. Feingold (1994) asserted that "we must reevaluate how we prepare future teachers, exercise leaders, and doctorates. The world is changing, society has different needs, and multidisciplinary perspectives, integrative approaches, and connections to societal needs all require a change in how we are preparing professionals" (p. 364). Accordingly, researchers must continue to investigate the relationship between the subdisciplines and the teaching of physical education in order to identify the subject matter and instructional methods that should be incorporated in the PETE curriculum.

\section{Delphi Method: A Tool for Multi-Disciplinary Research and Communication}

The complex nature of the theory to practice dilemma in PETE mandates the use of an alternative research design that provides a systematic protocol for enhanced multi-disciplinary communication. Any decision-making process regarding the theory to practice transition within the PETE curriculum must incorporate individuals from multiple backgrounds, perspectives, and disciplinary orientations (Sahakian, 1997). The Delphi Method is one research technique that has been endorsed for synthesizing expert judgments and deriving consensus (Gordon, 1992), in situations where group meetings are considered to be impractical due to the logistical or methodological limitations commonly associated with face-to-face discussion (Clayton, 1997). Additionally, the Delphi Method has been recommended for use when the complexity or ambiguity associated with a particular problem exceeds the intellectual capabilities of a single decision-maker (Chiappetta \& Fillman, 1998; Clayton, 1997; Jeffery \& Hache, 1995; Sahakian, 1997). The underlying assumption of the Delphi Method is that the informed, collective judgment of a group of experts is more accurate and reliable than individual judgment (Clayton, 1997; Gordon, 1992; Ziglio, 1996) in dynamic environments where effective decision-making is dependent on the "knowledge, resourcefulness, expertise and creativity [of] different types of people" (Sahakian, 1997, p.2).

Ziglio (1996) described the Delphi Method as "a structured process for collecting and distilling knowledge from a group of experts by means of a series of questionnaires interspersed with controlled opinion feedback" (p. 3). The Delphi Method has also been characterized as a systematic, effective, and comprehensive technique for administering a group communication process that enables a collection of knowledgeable individuals to reach a consensus of opinion in circumstances when the available information is relatively incomplete or multi-disciplinary in nature (Clayton, 1997; Gillespie \& Lowe, 1995; Jeffery \& Hache, 1995). The following sections provide textual support for the methodology employed in this study and a brief review of the literature pertaining to the (a) objectives, (b) research protocol, (c) advantages, and (d) limitations commonly associated with the use of the Delphi Method.

\section{Delphi Method Objectives}

The Delphi Method was initially developed by Norman Dalkey and Olaf Helmer at the RAND Corporation during the 1950's and 1960's for the purposes of military technology forecasting (Gordon, 1992; Pollard \& Tomlin, 1995; Sahakian, 1997), information gathering (Jeffery \& Hache, 1995), and group decision-making (Stahl \& Stahl, 1991). Subsequently, the Delphi Method has been implemented for a variety of educational applications including curriculum development, institutional planning, distance education, competency evaluation, instructional assessment, teacher education, and so forth (Clayton, 1997). Researchers have also employed the Delphi Method for organizational goal setting, clarifying alternative action plans, setting priorities, information gathering, and educating respondent groups (Linger, 1998). Researchers in higher education have primarily used the Delphi Method in the following four areas: "(1) to develop goals and objectives, (2) to improve curriculum, (3) to assist in strategic planning, and (4) to develop criteria" (Murry \& Hammons, 1995, p. 425). More specifically, LaPlante (as cited in LaPlante \& Jewett, 1987) concluded that the Delphi Method is an appropriate tool 
for investigating the physical education curriculum. Subsequently, the Delphi Method has been employed in a wide variety of physical education studies (see Table 1).

As illustrated by the previously discussed areas of application, the Delphi Method has proven to be an extremely valuable tool for educational planning and decision-making under a wide range of circumstances (Murry \& Hammons, 1995; Pollard \& Tomlin, 1995). The Delphi Method is considered to be most useful, however, in situations when precise analytical study is not possible due to the uncertain or ambiguous nature of the research problem (Baldwin-Morgan, 1993; Murry \& Hammons, 1995; Sahakian, 1997; Ziglio, 1996). Ziglio (1996) reported that an individual may select from the following two courses of action when the problem-solving process is dependent upon inadequate information and incomplete theory:

The first option is to wait (perhaps indefinitely) until we have an adequate theory based on tested scientific knowledge enabling us to address the problem concerned. Of course, this option is not feasible if the problem needs urgent attention and action. Furthermore, many social and health problems are not amenable to solution by pure positivistic or 'scientific' methods (Goldschmidt, 1975, p. 199). The second alternative is to make the most of what is, admittedly, an unsatisfactory situation, and to try to obtain the relevant intuitive insights of experts and use their informed judgment as systematically as possible. According to Dalkey (1967), the rationale and use of the Delphi Method represents a systematic effort within the second alternative. (p. 4)

Additionally, the Delphi Method's use is predicated on the underlying assumption that the informed judgment from a group of experts regarding a complex problem is likely to be more reliable and accurate than the judgment of a single individual or group of non-experts (Sahakian, 1997; Ziglio, 1996). Murry and Hammons (1995) reported that the Delphi Method could be effectively implemented as a valid research technique in situations where (a) the logistical constraints make repeated multiple group meetings infeasible, (b) the heterogeneity of the participants must be maintained to ensure validity of results, (c) the individuals needed to contribute have diverse backgrounds and no established history of communication, (d) the group process must incorporate too many individuals for a face-to-face group exchange, and (e) the disagreements among individuals are "so severe or politically unpalatable that the communication process must be refereed and/or anonymity assured" (p. 428).

The research objectives that are commonly associated with the Delphi Method remain intimately connected to the distinctive rationale that underlies this group decision-making process. The three fundamental objectives of the Delphi Method have been summarized as (a) the development of a range of responses to a particularly problematic issue, (b) the ranking of a range of responses in order to provide an indication of significance, and (c) the establishment of consensus regarding a range of responses (Jeffery \& Hache, 1995). Similarly, Stahl and Stahl (1991) identified the following possible objectives of a Delphi investigation:

1. Identifying and investigating underlying assumptions that contribute to divergent judgments or opinions.

2. Ascertaining information that may help to generate a consensus of opinion from a selected panel of experts.

3. Establishing relationships between expert judgments in the form of rankings on a topic that pertains to a number of disciplines. 
4. Educating the respondent group to the diverse and multi-disciplinary nature of the topic in question.

\section{Delphi Method Protocol}

The research protocol associated with the Delphi Method has been characterized as a highly flexible problem-solving process that provides "an opportunity for structured communication, by which expert panel members could provide feedback, revise judgments, and contribute to the development of agreedupon practices - all with complete anonymity" (Flippo, 1998, The Study section, \2 ). The following characteristics are recognized as critical components of the Delphi Method: (a) the reliance on the informed judgment of a carefully selected panel of experts, (b) the utilization of multiple surveys, (c) the asynchronous and anonymous participation of the Delphi panel members, (d) the establishment of consensus through the reporting of feedback regarding the responses from the previous round, and (d) the provision of direction and control to the research process by a Delphi manager (Gordon, 1992; Jeffery \& Hache, 1995; Sahakian, 1997; Stahl \& Stahl, 1991; Ziglio, 1996).

Delphi panel. The selection of panel members is considered to be a critical component of the Delphi Method that is directly related to the focus or objectives of the investigation (Gordon, 1992; Stahl \& Stahl, 1991). While considerable variation of opinion exists regarding the ideal size for a Delphi panel, the literature suggests that the panel should include at least 10 members (Parentè \& Anderson-Parentè, 1987) but little improvement in results can be expected as the panel increases beyond 25-30 members (Brooks, 1979; Delbecq, Van de Ven, \& Gustafson, 1975). A Delphi panel typically consists of 15-30 individuals from a specific homogenous population and 5-10 individuals from a heterogeneous population with a different level of expertise and social/professional stratification (Clayton, 1997) who have displayed a high level of knowledge and practical engagement with the problems that are currently being studied (Ziglio, 1996).

Delphi panel members are usually identified through literature searches and recommendation from other recognized experts in the field (Gordon, 1992). It is strongly recommended, however, that the researcher adhere to a specific set of criteria, rather than mere personal preference when selecting prospective experts to serve on the Delphi panel (Ziglio, 1996). Furthermore, it is not unusual for Delphi panels to include individuals with varying degrees of expertise in a particular area (Stahl \& Stahl, 1991).

Questionnaire design and administration. The Delphi Method involves the circulation of three or four questionnaires consisting of a number of items regarding a specific topic of interest (Stahl \& Stahl, 1991). Statements regarding the topic are generated based on the available literature and the initial opinions of a carefully selected panel of experts. Each member of the panel is asked to respond to each statement in questionnaire form in accordance with their own personal knowledge and perceptions. The results of each round of anonymous questioning are summarized and shared with the intention that panel members will reconsider those responses that deviate significantly from the group's overall mean ranking. Ziglio (1996) stated that "during this interactive process, which can be repeated as many times as are judged appropriate in the circumstances, issues can be clarified, areas of agreement and disagreement can be identified, and an understanding of the priorities can be developed" (p. 9).

The Delphi process traditionally incorporates two primary investigative phases (Ziglio, 1996). The exploration phase characterizes the first one or two rounds of questionnaire distribution where the issues being investigated are explored fully by the participating panel members. The evaluation phase involves all subsequent rounds of investigation for the purpose of evaluating the issues identified in the previous exploration phase. Throughout this highly interactive process, it is important to remember that various features of the research protocol, such as questionnaire design, the use of measurement scales, and the provision of feedback can greatly influence the communication among panel members and the eventual 
outcome of the study. Accordingly, the survey instrument must be subjected to rigorous pre-testing and the procedures for the provision of feedback should be carefully specified.

The Delphi Method's ultimate purpose is to facilitate in-depth conversation among a group of experts by providing them with opportunities to develop a more complete understanding of their peer's respective opinions, assessments, and forecast assumptions regarding a particularly problematic issue (Ziglio, 1996). Accordingly, the precise methodology employed during a Delphi investigation may vary depending on the focus of the research (Flippo, 1998). This high degree of flexibility is inherent to the Delphi Method because it enables researchers to focus and guide the communication process as it pertains to a wide array of problems, disciplines, levels of expertise, and so forth. The modified Delphi Method is one design variation that is particularly noteworthy. During a modified Delphi investigation, the researcher provides the panel members with an initial list of statements to be critiqued, and eliminates the traditional openended questionnaire which is generally used during the first round of surveying (Murry \& Hammons, 1995; Williams, 1998). The modified Delphi Method expedites the investigative process and enables the researcher to maintain control over the range and scope of the issues that are being discussed.

Asynchronous and anonymous participation. The asynchronous and anonymous participation of the panel members represents a defining characteristic of the Delphi Method. The asynchronous nature of the Delphi Method refers to each panel member's right to individually select the occasions when they will participate in the group communication process (Ziglio, 1996). Additionally, the panel members may elect to only respond to those items of the problem that they feel best qualified to address.

The use of anonymous, sequential questionnaires is intended to facilitate the equal participation and involvement of all panel members. Anonymity eliminates numerous communication barriers that are necessarily inherent to face-to-face meetings, such as conflicting personalities, organizational hierarchy, political factors, presentation skills, and strong individual wills (Sahakian, 1997). The removal of these potentially inhibiting variables enables each member of the group to contribute fully and truthfully with the knowledge that their ideas will be evaluated on the basis of merit rather than personality, reputation, or position.

While the value of anonymous participation remains central to the Delphi Method's success, it has been suggested that this anonymity "can cause participants to feel isolated and make it difficult for them to judge how best to formulate their ideas so that others will understand them" (Rotondi \& Gustafson, 1996, p. 38). A number of solutions have been recommended to prevent this communication barrier from occurring throughout the duration of a Delphi investigation. Team building techniques, straw model construction, and reduced participant anonymity have all been advocated as alternative methods for reducing participant isolation and enhancing in-depth conservation among panel members. For example, biographical sketches of each panel member can be shared prior to the initiation of the group communication process in order to provide the participants with a deeper understanding of the individuals they will be interacting with.

Supervision and feedback. The provision of researcher-directed statistical group responses and feedback regarding each round of questioning represents another defining characteristic of the Delphi Method (Murry \& Hammons, 1995). Typically, the Delphi panel members are required "to consider, to rank and/or rate, to edit, and to comment upon the responses developed during round one" (Murry \& Hammons, 1995, p. 424). The researcher then tabulates the results of each round of questioning and provides the panel members with the frequency distributions, means, and standard deviations for each item on the questionnaire. Additionally, a complete list of panel member comments could be provided with successive iterations (Clayton, 1997). This cyclical process of questioning, reconsideration, and feedback is continued until a convergence of panel member responses takes place (Murry \& Hammons, 1995). 
Group consensus. As previously discussed, the Delphi Method is particularly useful for eliciting and combining expert judgments rather than factual information (Gordon, 1992). In many instances, the primary objective of the Delphi investigation is to establish some measure of consensus regarding the panel members' opinions (Jeffery \& Hache, 1995). The reliance on small, non-representative samples associated with most Delphi investigations prohibits the utilization of inferential statistics (Gordon, 1992). Gordon theorized that the true value of the Delphi Method concerns the generation of ideas rather than the determination of statistically significant results that can be generalized to a larger population.

Unfortunately, the literature provides little direction concerning the determination of consensus because there is currently no agreement regarding the designation of a minimum percentage of response needed to demonstrate consensus (Murry \& Hammons, 1995). Brooks (1979) suggested a generalized definition of consensus as the "gathering of individual evaluations around a median response, with minimal divergence" (p. 378). Linger (1998), for example, proposed that a consensus of agreement was achieved when an item obtained a mean rating of five of higher on a seven-point Likert scale, with at least seventy percent of the participants rating the item as a five or higher.

\section{Advantages of the Delphi Method}

The distinct methodological characteristics of the Delphi Method contribute greatly to its usefulness as a research instrument in policy decision-making and long-range forecasting (Gordon, 1992). The most significant benefit of the Delphi Method concerns participant motivation. When conducted properly, the Delphi Method enables the research participants to assume ownership of a particular problem and its accompanying solution (Sahakian, 1997). This sense of personal ownership theoretically translates into a more effective and expedient resolution to the problem.

Additional advantages of the Delphi Method include the following: (a) the improved accuracy of the decision-making process due to the use of controlled-feedback and anonymity, (b) the elimination of the geographical and logistical impediments inherent to face-to-face group meetings, (c) the establishment of consensus based on the group's systematic evaluation, reflection, and reevaluation of the pertinent issues, and (d) the statistical description of the group responses (Murry \& Hammons, 1995). Ziglio (1996) reviewed the relevant literature and argued that the strengths of the Delphi Method pertained most directly to its ability to focus attention on the most relevant issues, provide a framework for group communication among individuals with divergent backgrounds and geographical locations, minimize the psychological and professional barriers to communication that are inherent to face-to-face meetings, provide an equal opportunity to respond for all the participants, and produce a detailed record of the decision-making process and the resultant informed judgment.

\section{Limitations of the Delphi Method}

Despite the proposed benefits of a group decision-making process, critics have raised several concerns related to the sampling and data analysis techniques associated with the Delphi Method (Sackman, 1974). While most of these criticisms regarding the scientific rigor of the Delphi Method have been satisfactorily addressed in the literature (Clayton, 1997; Goldschmidt, 1975), it is essential that the researcher acknowledge and account for the remaining limitations in his or her research design.

Jeffery and Hache (1995) suggested that the Delphi Method is not an effective research tool for routine decision-making due to the time-consuming nature of the communication process. The time delays between rounds may prove to be particularly problematic if the research involves nonprofessional or young respondents. The multiple rounds of questionnaire circulation that are typically employed greatly increase the required time for completion of the study. Advances in computer technology, 
however, might eventually eliminate this restriction and allow for the simultaneous input from multiple participants in the areas of idea generation, analysis, and evaluation (Gordon, 1992; Sahakian, 1997; Turoff \& Hiltz, 1996).

Murry and Hammons (1995) suggested that the questions formulated by the researcher could possibly influence the panel members' responses and that the researcher can never be completely certain that the participants' fully comprehend the purpose of the study. Furthermore, the researcher may never be able to evaluate and access each panel member's full expertise due to the absence of face-to-face meetings. As with other forms of survey research, participant motivation and non-response rate or sample attrition remain primary concerns as well.

Clayton (1997) summarized the primary methodological limitations associated with the Delphi Method in the following list:

1. The personal backgrounds and experiences of the panel members are generally beyond the control of the researcher.

2. The panel members' personal and professional responsibilities may limit the amount of time and effort that each individual can invest in the decision-making process.

3. The process by which the panel arrives at consensus remains largely unknown. It is uncertain whether the panel members alter their decision-making process as a result of careful reconsideration or the pressure to conform.

4. The results of a Delphi Method cannot be generalized beyond the specific panel of experts that participated in the study. Additionally, the strength of the findings depends largely on the backgrounds and perceptions of the panel members.

\section{Summary}

Despite the previously mentioned limitations, the Delphi Method represents an important potential resource for facilitating communication between exercise science specialists, teacher educators, and practicing physical educators. This type of systematic communication may represent an important first step in bridging the gap that currently exists between the foundational subdisciplines and the practice of teaching physical education in a school-based setting. The ideas that are generated as result of the Delphi process may ultimately help to determine future instructional practices in PETE and increase the prospective physical educator's ability to teach the cognitive, affective, and psychomotor skills that will enable his or her students to maintain physically active lifestyles throughout adulthood. 


\section{CHAPTER III METHODOLOGY}

The purpose of this study was (a) to generate a consensus of expert judgment regarding the critical theoretical and applied exercise science competencies that prospective physical educators need to learn within the physical education teacher education (PETE) curriculum and (b) to generate a list of recommended instructional methods for the delivery of exercise science content to prospective physical educators. The research methodology that is described in this chapter includes the following sections: (a) Participants, (b) Research Design, (c) Instrumentation, and (d) Administrative Procedures.

\section{Participants}

The initial step of a Delphi investigation involves the identification and selection of the expert panel members. Clayton (1997) stated that "the process of selecting experts is critical to the Delphi and serves to authorise the Delphi's superiority and validity over other less painstaking and rigorous survey procedures" (Selection of Expertise section, 13 ). The most significant concern in this selection process relates to the prospective panel members' level of expertise regarding the specific area of questioning. Murry and Hammons (1995) explained that "expertise implies that the individual panelists have more knowledge about the subject matter than most people, or that they possess certain work experience, or are members in a relevant professional association" (p. 428). It is also recommended that the majority of Delphi panel members come from a homogenous population within the same discipline, while incorporating a smaller number of participants with expertise on a particular topic from different social or professional stratifications (Clayton, 1997). While considerable variation of opinion exists regarding the ideal size for a Delphi panel, the literature indicates that the expert panel should include a minimum of at least 10 members (Parentè \& Anderson-Parentè, 1987) but little improvement in results can be expected as the panel increases in size beyond 25-30 members (Brooks, 1979; Delbecq, Van de Ven, \& Gustafson, 1975).

The purpose of this Delphi study was to investigate the theoretical and applied value of exercise science in the preparation of prospective physical educators to promote physically active lifestyles among children and youth within a school-based context. In order to effectively address this issue, the Delphi panel needed to include individuals with expertise in exercise science, teacher education, and the actual practice of teaching physical education. Based on the previously described recommendations regarding Delphi panel selection, a group of 20 experts was randomly selected and recruited from three pools of qualified candidates representing each of the following levels of expertise:

1. Exercise science specialists $(n=10)$ who currently served as faculty members at Research I universities and were listed as members of the Exercise Physiology Academy of the National Association for Sport and Physical Education.

2. Physical education teacher educators $(n=7)$ who currently served as faculty members at Research I universities and were listed as members of the Curriculum and Instruction Academy of the National Association for Sport and Physical Education.

3. Physical education teachers $(n=3)$ who had completed the Physical Best Health-Fitness Instructor Certification workshop through the National Association for Sport and Physical Education.

See Table 2 for a list of the Delphi panel members along with their areas of specialization and institutional affiliation. 


\section{Research Design}

The complex nature of the theory to practice dilemma in PETE mandates the use of an alternative research design that provides a systematic protocol for enhanced multi-disciplinary communication. The Delphi method was developed initially at the RAND Corporation in the 1950's as a means for collecting and organizing expert opinion concerning U.S. strategic defense planning (Clayton, 1997; Murry \& Hammons, 1995; Stahl \& Stahl, 1991). Subsequently, researchers have used the Delphi method to generate ideas and forecast changes related to a wide variety of educational issues (Clayton, 1997). Pollard and Tomlin (1995) stated that "by providing for the systematic solicitation and collection of opinions, the Delphi enables researchers to gain a consensus from a group of knowledgeable people about a particular topic without the time and expense involved in bringing them together" (Methodology section, \3).

This research project involved a two-round modified Delphi protocol as a means to determine a final consensus regarding the critical exercise science competencies and instructional methods that should be included in the PETE curriculum. The literature supports that two rounds of questioning are typically adequate for the determination of consensus (Murry \& Hammons, 1995). During each round of questioning, the panel members were required to rate a series of exercise science competencies in terms of their theoretical importance and pedagogical relevance for prospective physical educators. For the purpose of this study, importance referred to the item's value as a theoretical underpinning of the physical education teaching profession. Relevance referred to the item's practical or applied significance in relation to the administration of a K-12 physical education program. During the second round, the panel member's were also asked to recommend four instructional methods for effectively delivering exercise science content within the PETE curriculum.

\section{Instrumentation}

The identification of critical exercise science competencies and recommended instructional strategies represented the primary objectives of this Delphi investigation. The instrument was derived from the current list of knowledge, skills, and abilities recommended by the American College of Sports Medicine (2000) for individuals working in the field of exercise science. These competencies represent the basis for the American College of Sports Medicine's highly regarded Health/Fitness Instructor certification, and are grouped into the following general categories: (a) anatomy/biomechanics, (b) exercise physiology, (c) human development/aging, (d) pathophysiology/risk factors, (e) human behavior/psychology, (f) health appraisal/fitness testing, (g) safety/injury prevention/emergency care, (h) exercise programming, (i) weight management, and (j) program administration. While the Delphi panel members were being recruited for participation in the study, a draft of the questionnaire items was designed and pilot tested (see Appendix A).

A panel of six reviewers with demonstrated expertise in the area of investigation were randomly selected from the pool of prospective Delphi panel members and used to evaluate the initial questionnaire items for content validity and completeness. The reviewers were asked to rate the content validity of 206 exercise science competencies using a five-point Likert scale. A rating of five or four indicated that the item was a valid theoretical or applied exercise science competency that could potentially be incorporated within the PETE curriculum. A rating of three meant that the respondent was neutral or undecided. A rating of two or one meant that the item should be eliminated from the final version of the questionnaire

because it lacked validity. Respondents were also required to add any items that would provide a higher degree of content validity, keeping in mind the ultimate goal of producing a comprehensive list of theoretical and applied exercise science competencies that could potentially be incorporated into the PETE curriculum. 
The data collected during the pilot study were used to formulate a list of exercise science competencies to be included on the final version of the questionnaire using the following criteria: (a) The item needed to receive a mean rating of at least three or higher in the area of content validity; and (b) at least four of the six judges needed to rank the item as a three or higher. A total of ten items failed to meet these criteria and were not included on the final version of the questionnaire. The eliminated competencies addressed the effects of specific medications on exercise prescription (e.g., antianginals, antihypertensives, antiarrhythmics), and some of the managerial issues more commonly associated with the operation of a commercial or corporate health fitness facility (e.g., program budgets, marketing, membership sales and retention). If any one expert reviewer recommended the addition of a new competency, it was added to the survey in the interest of compiling the most inclusive list possible. A total of 26 new items were added to the initial list of competencies based on reviewer recommendations. These newly added items related to the areas of developmental exercise physiology, special populations, psychology of exercise adherence, and field-based physical fitness assessments.

The resulting 222 items were organized into a questionnaire format that required the actual Delphi panel members to rate each item regarding its theoretical importance within the PETE curriculum and pedagogical relevance concerning the instruction of physical education in a school-based setting. Each item's importance was rated using the following five-point Likert scale: (5) Strongly Agree, (4) Agree, (3) No Opinion, (2) Disagree, and (1) Strongly Disagree. The determination of pedagogical relevance involved the use of a similar Likert scale. An additional section was added to the second round questionnaire requiring the panel members to describe four recommended instructional methods for the delivery of exercise science in the PETE curriculum.

\section{Administrative Procedures}

The administrative procedures described in this section have been adapted from the generalized research protocol described by Jacobs (1996). These administrative procedures are described in the following sections: (a) Selection of Participants, (b) Round I Procedures, and (c) Round II Procedures.

\section{Selection of Participants}

Delphi panel members were personally invited to participate in the study through a pre-notification e-mail and follow-up telephone interview. A phone script describing (a) the purpose of the study, (b) an explanation of the Delphi Method, (c) an explanation of the time requirements, and (e) an invitation to participate was developed and used to contact prospective panel members (see Appendix B). The lead researcher also used that telephone call to answer any additional concerns expressed by the prospective panel members and to reinforce the significance of their potential contribution to the study. The initial list of prospective panel members included 40 exercise science specialists, 73 teacher education specialists, and 96 physical education teachers. Seven exercise science specialists, six teacher education specialists, and three physical education teachers turned down invitations to serve on the Delphi panel. After each refusal another name was randomly selected from the appropriate list until panel selection was completed

Jacobs (1996) reported that participant attrition is a primary concern during the administration of a Delphi study due to the voluntary nature of participation and the use of multiple rounds of questionnaire circulation during the data collection process. In order to ensure an appropriate response rate, the individuals who agreed to serve on the Delphi panel were provided with a small honorarium as an incentive for their participation. As recommended by Jacobs, a preset experimental mortality standard was also employed to determine the deleterious effects of participant attrition on the results of the study. The results of the study would be considered compromised if $20 \%$ of the panel members who returned the Round I questionnaire failed to complete the Round II questionnaire. All 20 Delphi panel members 
completed both rounds of questionnaire circulation, so participant attrition was not considered to be a negative factor.

\section{Round I Procedures}

After the approval from the Institutional Review Board for the Protection of Human Subjects was obtained, the survey instrument design was completed, and the selection of participants had been finalized, each participant received the first mailing of the questionnaire (see Appendix B). This mailing included a cover letter that (a) thanked them for agreeing to participate in the study, (b) restated the purpose and rationale of the study, (c) explained their rights and responsibilities as research participants, and (d) provided instructions for completing the questionnaire. The mailing also included a copy of the first round questionnaire and a self-addressed return envelope.

Within two weeks after the first round mailing, each Delphi panel member received a follow-up email and telephone call to confirm receipt of the mailing, answer any remaining questions, and prompt the timely return of the questionnaire. The participants who failed to return the survey within four weeks of the mailing date were (a) contacted by e-mail and telephone to reiterate the importance of the study, (b) issued a second copy of the survey packet if requested, and (c) encouraged to return the questionnaire as soon as possible. Following the receipt of all Round I questionnaires, participant responses were recorded in an electronic database and the mean group rating was calculated for each item.

\section{Round II Procedures}

The second round of questioning adhered to the same administrative procedures described in the previous section. This mailing included (a) a cover letter that restated the purpose and significance of the study, (b) the instructions for completing the questionnaire, (c) the second round questionnaire, and (d) a self-addressed return envelope (see Appendix C). A summary of the both the individual and group mean ratings from the previous round of questioning was incorporated as a component of the second round questionnaire. The panel members were asked to reevaluate their individual Round I responses based on the mean group ratings for each questionnaire item. The administrative procedures for the return of the questionnaires, provision of feedback, and data recording were identical to those described for the first round of investigation. An additional section was added to the second round questionnaire requiring the panel members to recommend four effective instructional methods for delivering exercise science within the PETE curriculum.

As an important last step in the research protocol, a final research report was sent to each Delphi panel member following the return of all Round II questionnaires and the completion of data analysis. The panel members were also thanked for providing the time and expertise required to complete this rather time-consuming process.

\section{Data Analysis}

The ultimate value of the Delphi Method relates to its ability to generate ideas regarding possible solutions to complex theoretical issues or problems. Accordingly, the expected outcomes for this research project included the generation of a list of theoretical and applied exercise science competencies this particular panel of experts regard as being critically important and pedagogically relevant for prospective physical education teachers. Additionally, the expert panel members were asked to recommend instructional methods for the delivery of exercise science content within the PETE curriculum. 


\section{Exercise Science Critical Concepts}

The data collected during the second round of questioning were used to provide a final measure of consensus regarding the critical strength of each exercise science competency. For this study, an item needed to be rated both sufficiently important and pedagogically relevant in order to be considered critical in nature. Each item had to meet the following criteria for consensus (Jacobs, 1996): (a) The item received a mean rating of at least four or higher in the areas of importance and relevance; and (b) the item received at least $75 \%$ of all individual ratings at the four level or higher. Any item that failed to meet this criterion was considered to be non-critical in nature.

\section{Exercise Science Instructional Methods}

During the second round of questioning, the participants also completed an attachment requiring them to describe recommended instructional methods for delivering exercise science as a component of the PETE curriculum. A content analysis was used to examine the Delphi panel members' responses to these open-ended questions regarding recommended instructional methods (Fleming \& Monda-Amaya, 2001). Emergent themes were identified and responses were sorted by both the researcher and by an independent rater. A point-by-point agreement ratio (Kazdin, 1983) of 91.30\% was established between the primary researcher and independent rater ([agreement/(agreements + disagreements)] x 100). 


\section{CHAPTER IV \\ RESULTS}

The purpose of this study was (a) to generate a consensus of expert judgment regarding the critical theoretical and applied exercise science competencies that prospective physical educators need to learn within the physical education teacher education (PETE) curriculum and (b) to generate a list of recommended instructional methods for the delivery of exercise science content to prospective physical educators. For this study, an item needed to be considered both sufficiently important and pedagogically relevant in order to be considered critical in nature. Each item had to meet the following criteria for consensus (Jacobs, 1996): (a) The item received a mean rating of at least four or higher in the areas of importance and relevance; and (b) the item received at least $75 \%$ of all individual ratings at the four level or higher. Any item that failed to meet these criteria was considered to be non-critical. Following the first round of questionnaire circulation, 101 competencies were rated as being of critical importance and pedagogical relevance. Following the second and final round, 109 competencies met the previously described criteria. Only the second round analysis is presented in this chapter, because it is the final round which is reflective of group consensus. The results are presented in the following categories: (a) Anatomy and Biomechanics, (b) Exercise Physiology, (c) Human Development and Aging, (d) Pathophysiology and Risk Factors, (e) Human Behavior and Psychology, (f) Health Appraisal and Fitness Testing, (g) Safety, Injury Prevention, and Emergency Care, (h) Exercise Programming, (i) Nutrition and Weight Management, (j) Program Administration and Management, and (j) Recommended Instructional Methods.

\section{Anatomy and Biomechanics}

The expert panelists reached a consensus of agreement regarding 10 of the 23 anatomy and biomechanics competencies that were included on the questionnaire (see Table 3 ). The competencies considered to be most valuable reflected the need for PETE students to possess a basic, rather than indepth, knowledge of anatomy and biomechanics: (a) knowledge of anatomy as related to exercise and health, (b) knowledge of biomechanics as related to sport and exercise participation, and (c) knowledge of the basic anatomy of the cardiovascular and respiratory systems. A consensus of agreement was reached concerning more applied anatomy and biomechanics competencies as well: (a) knowledge of functional anatomy and biomechanics, (b) knowledge and skill to demonstrate resistance training exercises, (c) knowledge and skill to demonstrate flexibility exercises, (d) ability to locate anatomical landmarks for skinfold and circumference measurements, and (e) knowledge of the biomechanical principles that underlie the performance of various physical activities including walking, jogging, swimming, cycling, and so forth. A developmental competency describing the effects of weight-bearing physical activity on optimal bone growth and development in children and adolescence was also rated as having critical importance and relevance.

Eight of the remaining items satisfied the required criteria for theoretical importance, but were not considered to be highly relevant for those preparing to teach physical education in school-based settings. Less agreement was obtained on those items which described highly specific anatomical and biomechanical terminology and concepts. For example, competencies related to (a) the basic structures of bone, skeletal, muscle and connective tissues, (b) the definition of biomechanical terminology (e.g., supination, pronation, flexion, extension, abduction, adduction), and (c) the knowledge to explain the myotatic stretch reflex failed to meet the pre-established criteria for consensus.

\section{Exercise Physiology}

The Delphi panel members reached a consensus of agreement regarding 17 of the 35 exercise physiology competencies that were included on the questionnaire (see Table 4). These critical competencies included a broad range of basic exercise physiology concepts and applications including 
knowledge of: (a) the primary energy systems in aerobic and anaerobic metabolism, (b) the components of health-related physical fitness, (c) the acute and chronic responses to cardiorespiratory exercise, (d) the physiological adaptations and training variables associated with resistance training program design, (e) the basic training principles for exercise program design, (f) the methods commonly used for monitoring cardiovascular exercise intensity, and (f) the physiological significance of a properly designed warm-up and cool-down.

Eight of the remaining items met the required criteria for theoretical importance, but were not rated highly enough in the area of relevance to be considered critical. A number of competencies addressing muscle physiology (e.g., theories of muscular fatigue, delayed onset muscle soreness, muscle fiber type differentiation, sliding filament theory, basic mechanisms of muscular contraction) and cardiovascular physiology (e.g., anatomy and function of cardiac muscle, blood pressure responses with acute exercise, ventilatory threshold and its role in cardiovascular assessment) were not rated as critical. The exercise physiology competencies concerning individuals with chronic degenerative disease were also regarded as non-essential.

\section{Human Development and Aging}

The Delphi panel members reached a consensus of agreement regarding 6 of the 11 human development competencies that were included on the questionnaire (see Table 5). Several developmental exercise physiology competencies regarding maturational change, exercise program modification based on age-related differences, and safety considerations for pre-pubescent children and post-pubescent youth were rated as critical. Competencies addressing the health-related benefits of regular physical activity for people of all ages and the unique physiological adaptations to exercise training across the lifespan were also emphasized.

Only one competency in this category met the criteria for importance, but not relevance: The knowledge and ability to describe changes that occur in maturation from childhood to adulthood (e.g., skeletal muscle, bone structure, reaction time, coordination, maximal oxygen consumption, heat and cold tolerance). Several other competencies related to aging and exercise programming for older adults were also rated as non-critical.

\section{Pathophysiology and Risk Factors}

The expert panelists reached a consensus of agreement regarding only 3 of the 14 competencies related to pathophysiology and risk factors (see Table 6). The ability (a) to determine risk factors that can be modified by regular physical activity, (b) to discuss the influence of lifestyle factors on blood lipid profiles, and (c) to explain the risk factor concept of coronary artery disease were considered to be critically important and relevant. Four of the remaining competencies in the area of pathophysiology were judged to be important but not necessarily relevant to the teaching of physical education. The items that were not considered critical addressed the risk factors and degenerative processes associated with a number of other cardiovascular, pulmonary, metabolic, and musculoskeletal diseases along with the resultant implications for exercise professionals.

\section{Human Behavior and Psychology}

The Delphi panel established a consensus of agreement regarding 9 of the 17 competencies in the area of human behavior and the psychology of exercise (see Table 7). Knowledge of the psychological benefits associated with regular participation in physical activity was considered to be critical along with the ability to recognize the signs and symptoms of disordered eating. A number of the critical competencies in this area addressed the promotion of exercise adherence (e.g., strategies for facilitating 
behavioral change, techniques for developing positive attitudes and enhancing participant motivation, goals setting and behavioral contracts). Other critical competencies described the physical educators need to understand basic behavioral and educational theories that can be employed in physical activity promotion settings. Knowledge of the environmental determinants of physical activity (e.g., accessibility, neighborhood safety, television/computers) was considered critical, but knowledge of the psychosocial determinants (e.g., parental/peer influence, self-efficacy, self-perception) was rated highly in the area of importance while failing to meet the set criteria for relevance. Other competencies in the areas of health counseling, test anxiety, and sport psychology were rated as non-critical by the panel.

\section{Health Appraisal and Fitness Testing}

The Delphi panel members reached a consensus of agreement regarding 12 of the 32 health appraisal and fitness testing competencies (see Table 8). The critical competencies indicate that prospective physical educators need the knowledge, skills, and abilities to administer a variety of fitness assessments for cardiovascular endurance, body composition, muscular strength, muscular endurance, and flexibility. Furthermore, skill is needed instructing participants in the use of testing equipment and modifying test protocol to meet the needs of individuals with developmental differences. More specifically, physical educators must also understand how (a) to prepare children for fitness testing (e.g., test purpose, procedures, pacing), (b) to interpret both norm- and criterion-referenced tests, (c) to relate fitness testing data to national and state standards for physical education, and (d) to use technology for monitoring physical activity participation (e.g., heart rate monitors, pedometers).

A number of the non-critical competencies were related to procedures for pre-physical activity screening, medical referrals, relative and absolute contraindications to exercise testing, provision of informed consent, specific protocol for laboratory rather than field-based tests (e.g., graded exercise testing using cycle ergometers and treadmills), and the effects of various medications and drugs on exercise test performance (e.g., bronchodialators, hypoglycemics, psychotropics, vasodialators). Several competencies pertaining to (a) the importance of health history, (b) the value of medical clearance prior to exercise participation, (c) the skill to measure heart rate, blood pressure, and rate of perceived exertion during, and (d) the knowledge of the advantageous and disadvantages of various body composition assessment techniques were considered to be of theoretical importance but lacking in pedagogical relevance.

\section{Safety, Injury Prevention, and Emergency Care}

The Delphi panel rated 10 of the 16 competencies in the area of safety, injury prevention, and emergency care as critical (see Table 9). The duty to provide a safe exercise environment for children and adolescence encompasses the physical education teacher's knowledge of (a) appropriate emergency procedures and the associated legal implications, (b) protocol for administering first aid, (c) basic safety precautions undertaken in a group exercise settings, (d) potential risks associated with the use of contraindicated exercises, and (e) effects of the physical environment on physiological responses to exercise. Perhaps most notably, knowledge and skill in providing basic life support and maintenance of cardiopulmonary resuscitation (CPR) certification was also considered to be a critical competency for prospective physical education teachers.

The non-critical competencies involved the recognition of various physical and psychological overuse syndromes, the skill to prescribe exercises for those with low back pain, the role of equipment maintenance in the risk management process, and the ability to describe the potential musculoskeletal, cardiopulmonary, and metabolic risks associated with exercise participation. These types of competencies are probably more applicable to exercise professionals working with various special populations, such as 
athletes and older adults who are at increased risk for overuse injury and chronic degenerative disease respectively.

\section{Exercise Programming}

The expert panelists reached a consensus of agreement regarding 31 of the 52 exercise programming competencies that were included on the questionnaire (see Table 10). From a theoretical standpoint, physical educators need to understand the difference between the amount of physical activity needed to improve health and physical fitness. Additionally, physical educators should comprehend the basic training principals (e.g., progression, overload, specificity) and their relationship to exercise program design. A variety of exercise-related terms (e.g., concentric, eccentric, progressive resistance, sets, repetition) were also thought to be of potential theoretical value and practical significance. From a developmental perspective, physical education teachers should be able (a) to infuse individuals with different needs into a group exercise environment, (b) to understand the special concerns and guidelines for youth resistance training, and (c) to demonstrate knowledge of the published physical activity guidelines and recommendations for children, adolescents, adults, and older adults.

The majority of selected competencies in this area, however, pertain to the actual practical application of exercise science in an applied setting. Generally, prospective physical educators should learn (a) to properly design and sequence the component parts of an exercise session, (b) to develop an appropriate warm-up and cool-down, (c) to modify exercise techniques for individuals of various ages, fitness levels, and physical conditions, (d) to communicate effectively with participants during a group exercise session, (e) to demonstrate proper exercise technique and familiarity with a variety of group exercise formats, (f) to evaluate specific exercises for safety and effectiveness, and (g) to record exercise sessions and perform periodic fitness evaluations to monitor changes in fitness status.

Several competencies specifically related to cardiovascular endurance were also rated as critical by the Delphi panel members. In summary, physical educators should be able to prescribe cardiovascular exercise using the training variables of frequency, intensity, time, and type. The skill to teach participants to monitor exercise intensity through the use of target heart rate and rating of perceived exertion (RPE) was considered critical as well. Prospective physical educators should be able to recognize proper and improper cardiovascular exercise technique and design training sessions using various formats including interval, continuous, and circuit training programs.

The issue of muscular fitness, which encompasses muscular strength and local muscular endurance, is also addressed within a number of the selected exercise science competencies. Physical educators need the ability to design resistance training programs and teach a progression of resistance training exercises for enhancing muscular fitness and to recognize proper and improper techniques in the use of a range of resistance training modalities (e.g., free weights, partner-resisted, stability balls, resistance bands). Additionally, they need the skill to prescribe and teach appropriate flexibility exercises for improving range-of-motion with the major joints, tendons, and muscle groups.

\section{Nutrition and Weight Management}

The expert panelists reached a consensus of agreement regarding 9 of the 18 competencies concerning nutrition and weight management (see Table 11). In summary, physical educators should understand (a) the key terminology associated with body composition (e.g., obesity, overweight, percent fat, body fat distribution), (b) the relationship between body composition and optimal health, (c) the

USDA Food Pyramid, and (d) the importance of adequate daily caloric intake and the related guidelines for those participants desiring to gain or lose weight. It is essential that physical education teachers possess knowledge of the effects of diet plus exercise, diet alone, and exercise alone as possible 
intervention strategies for weight management as well. Panel members also rated the ability to describe appropriate hydration practices and discount inappropriate weight loss techniques as critical.

A number of more theoretically complex nutritional competencies, related to the role of specific nutrients (e.g., vitamins, minerals, carbohydrates, fats, and proteins) in the maintenance of a healthy body composition were not considered critical. The competencies that addressed nutrition and sport performance enhancement (e.g., ergogenic aids, female athlete triad syndrome) were also rated below the set criteria for consensus of agreement. Somewhat surprisingly, competencies describing body fat distribution patterns and the various professional association position statements on fitness and weight loss were regarded as being theoretically important, but pedagogically irrelevant.

\section{Program Management and Administration}

The Delphi panel rated 2 of the 4 exercise science competencies in the area of program management and administration as being critical (see Table 12). The Delphi panel confirmed that physical educators need the knowledge, skills, and abilities to administer and deliver health fitness programs. More specifically, the ability to advocate participation in regular physical activity through the development and delivery of educational programs (e.g., lectures, workshops) and educational materials (e.g., promotional flyers, brochures) is also necessary.

The two competencies that did not meet the designated criteria for consensus are probably more applicable to exercise professionals working in commercial, community, clinical, and corporate health fitness programs. The ability to create and maintain records pertaining to participant exercise adherence, retention, and goal setting was thought to be of potential theoretical importance but lacking in pedagogical relevance. Additionally, the ability to use documentation for physician referrals based on problematic signs and symptoms during exercise was not considered to be sufficiently important or relevant for prospective physical educators.

\section{Instructional Methods}

During the completion of the second round survey, the Delphi panelists were also asked to recommend four instructional methods for delivering exercise science content to prospective $\mathrm{K}-12$ physical education teachers. The 20 expert panel members recommended a total of 69 instructional methods for delivering exercise content within the PETE curriculum (see Figure 1). These recommendations were divided into the following response categories: (a) Teacher Directed Instruction, (b) Active Learning, (c) Lab Component, (d) Experiential Learning, (e) Technology Integration, and (f) Alternative Curricular Models.

\section{Teacher-directed Instruction}

Teacher-directed instruction is a widely recognized model of teaching that has received a considerable amount of support in the literature regarding its effectiveness in a variety of educational contexts (Brophy \& Good, 1986; Housner, 1990; Rosenshine \& Stevens, 1986). When using a direct instructional approach, the teacher typically exhibits the following behaviors: (a) communicate clear lesson objectives, (b) engage the students in a progressive series of learning activities that allow for a high rate of success, (c) employ a variety of teaching strategies, such as modeling and instructional cueing, to explain the lesson content, (d) ask frequent questions to monitor student comprehension, (e) provide continual performance-related feedback, and (f) re-teach the lesson content as needed (Housner, 1990).

The continued popularity of this instructional approach was reinforced by the fact that $17.39 \%$ (12 of 69) of the total responses in this study related to the use of teacher-directed approaches for delivering 
exercise science content to prospective physical educators, including lecture-discussion lesson formats, teacher/student demonstrations, and provision of regular feedback. These types of teacher-directed instructional methods were favored for their effectiveness, efficiency, and flexibility in delivering content to large groups of students. For example, one expert panelist stated that "the methodology, if conducted properly with a well trained specialist, has been effective over the years," and continues to be "the dominant method of disseminating knowledge (content) to interested, ready-to-learn" students. In addition to being an effective method for delivering theoretical exercise science subject matter, another respondent recommended direct instruction for preparing PETE students in the performance of specific exercises and the use of specialized equipment (e.g., resistance balls, elastic tubing, free weights, resistance training machines) that are commonly employed in school-based physical education programs.

\section{Active Learning}

Despite the substantial amount of empirical support concerning the effectiveness of a direct model of instruction, critics have argued that "direct instruction may not be as effective if the teacher's intent is to promote affective, social, or higher order cognitive growth" (Housner, 1990, p. 221) in learners. McBride (1999), for example, observed that teacher-centered learning environments in physical education offer few opportunities for students to think critically about their own learning. McBride suggested that teachers can restructure the teaching-learning environment to actively promote critical thinking among students by (a) assuming the role of facilitator rather than director, (b) using a variety of cooperative learning strategies, and (c) encouraging students to think reflectively about their cooperative learning experiences. When teachers implement active learning strategies, students are required to do most of the work by studying ideas, solving problems, and applying concepts in a supportive and personally challenging instructional climate (Silberman, 1996).

The concept of active learning encompasses a wide variety of student-centered activities that are intended to promote higher levels of student engagement and mastery of the involved course content. Sample active learning approaches include the use of collaborative group work, role playing, practice teaching opportunities, problem-based learning, case study analysis, student research projects, informational interviews with professionals, and so forth. In this study, $31.88 \%$ (22 of 69) of the recommended instructional methods related to the use of active learning as an important adjunct to more traditional, teacher-directed methods of instruction.

In justification of his recommendation, one panel member suggested that "once the students understand the basic facts and concepts, they must engage with the content. These methods promote discussion and expose students to a variety of different perspectives." Other panel members supported their recommendation by discussing the need (a) to account for various learning styles among students, (b) to motivate and engage students more completely in their intellectual development, (c) to afford students the opportunity to practice required skills in a well-controlled environment, (d) to further challenge students with "real life" situations or problems, (e) to foster collaborative relationships among peers, (f) to better integrate knowledge across disciplines, and (g) to promote effective communication, team work, and problem solving among students.

\section{Lab Component}

While lab experiences in exercise science were also widely endorsed for promoting active student engagement and learning, related responses were categorized separately for the purpose of this analysis due to the varied nature of the types of labs recommended by the Delphi panel members. A lab component was defined as any formal lab experience in an applied or clinical exercise science setting. These labs could potentially be stand alone educational experiences or supplements to existent lecture 
courses. In summary, $21.74 \%$ (15 of 69 ) of the recommended instructional methods pertained to the use of some form of exercise science lab component.

These responses reflected little consensus, however, regarding the type of lab experience that would prove most beneficial for preparing physical education majors. A number of the responses were very general and offered limited insight into what exactly this component of the PETE curriculum should entail. More specific recommendations indicated the need for traditional exercise physiology labs that enable students (a) to practice testing various components of health-related physical fitness, (b) to collect quantitative data for interpretation and analysis, and (c) to better understand basic exercise science principles within an experimental context. Several other respondents indicated the need for lab experiences that are more highly focused on exercise prescription and related to methods of teaching. This alternative type of lab experience "would include hands on teaching of group exercise as well as techniques to teach individuals how to work on health-related fitness components." Another panel member pointed out that "most physiology labs are conducted in an actual human performance lab, in this case, the lab would be the gymnasium or field."

\section{Experiential Learning}

Experiential learning was operationally defined as any type of internship, practicum, or field placement within an applied physical education or exercise setting. Gardner and Van der Veer (1997) recommended the use of experiential learning as an important instructional strategy for (a) facilitating students' transition to post-college life, (b) providing students with regular opportunities for meaningful self-reflection regarding their own professional readiness, and (c) helping to integrate and bring successful closure to the undergraduate experience. These important educational objectives were clearly reflected in a number of the Delphi panel members' responses in the area of experiential learning.

Of the total responses, $15.94 \%$ (11 of 69) concerned the use of experiential learning including (a) early practice teaching, (b) integrated field experiences across the curriculum, (c) field trips and observations in various exercise settings, and (d) actual teaching experience with children and youth. In promoting the need for early experiential learning opportunities, one panelist stated that "it is important for [PETE] students to see the dynamics of the job early in the program to better ensure their satisfaction with the curriculum and expectations." Several others also commented that the experiential component of a curriculum provides the student with the opportunity to learn how to be a professional. Another panel member suggested that in an experiential context, "learning tends to be more realistic, more meaningful and more effective toward launching young learners into an environment [physical education teaching] that may otherwise be unknown, untested or even threatening." Furthermore, the cooperative blending of school and university was perceived as a vehicle for enhancing the quality of physical education along with affording PETE students a more engaging teaching-learning environment.

\section{Technology Integration}

Recommendations regarding the use of instructional technology were limited to $10.14 \%$ (7 of 69) of the total responses. Recommended instructional methods in this category included the use of multi-media presentation software, electronic heart rate monitors, and internet-assisted instruction. Several panelists suggested the use of presentation software in the classroom (e.g., PowerPoint) for communicating "the information in a dynamic way using multiple sources of media." Another reiterated the need for prospective physical educators to become well rehearsed in the use of this type of presentation software as "the wave of the future - or actually the present." Internet-assisted instruction was recommended as a possible strategy for getting students more actively involved in their own learning and promoting higher levels of understanding, while allowing for asynchronous participation. One panelist indicated that "accessibility to and competence with current technology is a basic phenomenon as young students begin 
their education in contemporary America." Accordingly, faculty members need to continue to take advantage of the numerous e-learning and distance learning opportunities that have played an increasingly important role on college campuses. Faculty members can no longer afford to limit their "teaching station to the classroom using chalk and overhead. Through technology, the opportunities are both exciting and challenging." Only one panel member recommended the use of a discipline-specific technology (e.g., electronic heart rate monitors for measuring exercise intensity).

\section{Alternative Curricular Models}

One Delphi panel member offered two suggestions concerning the use of alternative curricular models for delivering exercise science content within the PETE curriculum. These recommendations for curricular revision represent only $2.90 \%$ ( 2 of 69 ) of the total responses, but support ideas that have been previously put forth in the literature. The panel member recommended merging exercise science concepts into a teaching methods course "so that students learn to actually apply the EP [exercise physiology] concepts in a classroom/gymnasium setting. In addition students would learn how to plan and deliver a fitness course" within a middle or high school physical education program. The second recommendation concerned development of an exercise physiology course specifically-designed for majors emphasizing pediatric, rather than adult, fitness concepts because "PETE students need information about children and youth." 


\section{CHAPTER V DISCUSSION}

The purpose of this study was (a) to generate a consensus of expert judgment regarding the critical theoretical and applied exercise science competencies that prospective physical educators need to learn within the physical education teacher education (PETE) curriculum and (b) to generate a list of recommended instructional methods for the delivery of exercise science content to prospective physical educators. This chapter includes a discussion of the following areas of potential interest: (a) Limitations of Delphi Method, (b) Exercise Science in PETE: Review, and (c) Exercise Science in PETE: Practical Implications.

\section{Limitations of Delphi Method}

As described in previous sections of this manuscript, the Delphi Method is a form of survey research that is most ideally suited for group decision-making about "complex or ambiguous problems that exceed the capabilities of a single person" (Sahakian, 1997, p. 1). Across academic programs and institutions, curricular decisions often involve complicated problems without clearly defined solutions. Faculty members with diverse backgrounds and interests must come to some form of consensus in addressing these curricular problems, while simultaneously taking into account a wide variety of philosophical and logistical considerations. Accordingly, the Delphi Method can be a useful tool in the cyclical process of curriculum design, implementation, and assessment.

As with any research methodology, however, Delphi practitioners must remember the associated methodological limitations throughout data collection, analysis, and interpretation. The reliability of a Delphi study, for example, is highly dependent on the qualifications and abilities of the selected panel of experts (Murry \& Hammons, 1995). The length of time needed to complete a Delphi study can also be prohibitive, leading to difficulty in recruiting a highly qualified panel of experts who are willing to commit the time and effort required to complete two to four rounds of survey iteration. Perhaps more significantly, even the most well conceived Delphi study may not yield "an exhaustive nor all-inclusive set of ideas" (Clayton, 1997, Conclusions section, 9 6). While a Delphi study may produce thoughtprovoking results, "the value of the information is for the reader to decide and is limited due to the constraints imposed by the panel selection, as well as by the backgrounds, experiences and biases of each member" (Clayton, 1997, Conclusions section, ๆ 6).

The results of a Delphi investigation are specific to the panel of experts and are not necessarily repeatable with other groups of similarly qualified members due to the considerable variation in individual backgrounds that exist. Furthermore, uneven numbers of representatives from each subgroup were used in this study to enhance the validity of the Delphi panel findings while still accounting for a relatively broad range of opinions across various levels of expertise in both the areas of exercise science and teacher preparation. The exercise science specialists, for example, were assumed to have the highest degree of subject matter expertise regarding the survey content and comprised the largest subgroup within the panel. The physical education teacher educators, who work with prospective physical educators on a daily basis, were assigned the second highest number of seats on the panel. The physical education teachers, whose practical experience in the field was critical to the credibility of the findings, were granted the fewest seats on the panel. It must be acknowledged that a different distribution of exercise science specialists, teacher educators, and physical education teachers could potentially reach another

distinct set of conclusions when presented with the same research questions. Future research could help to better generalize the results of this study by circulating a modified version of the employed questionnaire to a larger sample of exercise scientists, teacher educators, and physical education teachers. The use of a larger sample could also prove helpful in identifying any differences of opinion that may exist among these groups of experts. It would be potentially valuable to determine if any differences of opinion exist within groups as well. It seems reasonable to hypothesize that physical educators working at the 
elementary, middle, and secondary levels may have some significant differences of opinion regarding the exercise science competencies that are both critically important and pedagogically relevant.

With these basic methodological limitations and future research directions in mind, it is important to reinforce that this research project was not intended to generate an all-inclusive list of exercise competencies and recommended instructional methods that need to be uniformly adopted across PETE programs. Those truly important decisions are best left to faculty members working within individual PETE programs to develop curricular models that represent their unique programmatic mission, goals, and intended learning outcomes. Despite the previously described limitations, the Delphi Method represents an important potential resource for facilitating communication among different academic disciplines or specializations. As PETE programs continue to experiment with alternative pedagogical approaches and curricular models, the need for interdisciplinary research and enhanced cross-disciplinary communication becomes more apparent. This study was primarily intended to initiate a meaningful discussion among expert teacher educators, exercise scientists, and physical educators regarding the critical exercise science competencies and instructional methods that should be incorporated in the PETE curriculum.

\section{Exercise Science in PETE: Review}

This specific cross-disciplinary discussion is particularly significant because the physical education profession continues to maintain a highly publicized relationship with physical activity and fitness. Individual physical activity levels, health-related physical fitness, health risk factors, and attitudes towards physically active lifestyles endure as important areas of interest for physical education professionals. Teacher educators, researchers, and physical education practitioners have investigated a wide range of fitness related issues in the professional literature. PETE programs must continually use this expanding body of knowledge to facilitate the professional development of prospective physical educators who are well-prepared to teach and apply this information in school-based settings. The practical implications of this knowledge base in exercise science relate to the enhancement of sport performance, safety of exercise program participants, interpretation of research findings, advocacy of physical activity, and so forth (Van Donselaar \& Leslie, 1990).

Our growing appreciation for regular physical activity and its accompanying health-related benefits further exemplifies the need for physical educators who are adequately prepared in the foundational area of exercise science. Individuals of every age and background can expect to substantially improve their health and quality of life by incorporating a minimum of 30 minutes of moderate-intensity physical activity on all, or at least most, days of the week (U.S. Department of Health and Human Services, 1996). The primary benefits associated with participation in regular physical activity include reduced risk of premature death, cardiovascular disease, diabetes, colon cancer, obesity, orthopedic ailments, depression, and anxiety.

Despite these well-publicized health benefits, many American children and youths continue to lead sedentary lifestyles (U.S. Department of Health and Human Services, 1996). Public school may represent the ideal setting for preparing children to make health-enhancing decisions regarding their own physical activity levels. A number of professional and governmental agencies have acknowledged the potential importance of school physical education programs in the promotion and development of physically active lifestyles among children and youth (e.g., American Academy of Pediatrics, 1987; National Association for Sport and Physical Education, 2004b; U.S. Department of Health and Human Services, 1996).

This growing national concern with physical activity and its role in the maintenance of good health is one factor that should be used to help determine the course content and instructional methods employed within the PETE curriculum. The PETE curriculum must provide prospective teachers with the 
knowledge and skills required to effectively foster the development of the physical, cognitive, and affective characteristics that will enable a child to enjoy a physically active lifestyle into adulthood. Accordingly, physical educators must be adequately prepared to apply the subdisciplinary concepts that may enhance a child's appreciation for regular physical activity as a critical component of a healthy lifestyle. While guidelines for the promotion of physical activity in school physical education programs have previously been established (National Association for Sport and Physical Education, 2004b), the preparation of prospective physical educators to meet these professional responsibilities remains an area of concern.

Through a systematic process of expert review, this study has generated a list of exercise science competencies and recommended instructional methods that relate directly to the prospective physical educator's ability to promote health-enhancing levels of physical activity and fitness within the context of a school-based physical education program. The results of this scholarly interaction have hopefully provided a conceptual framework upon which PETE faculty members can make future curricular decisions in the area of exercise science.

\section{Exercise Science PETE: Practical Implications}

The transfer of exercise science theory into professional practice represents a potentially problematic issue for physical education teacher educators (Miller \& Housner, 1996; Van Donselaar \& Leslie, 1990).

The general categories related to exercise science course content and recommended instructional methods generated during this Delphi investigation are graphically depicted in Figure 2.

\section{Exercise Science Course Content}

During the 1960's and 1970's, PETE programs embraced a curricular orientation that placed a greater emphasis on subdisciplinary knowledge (Corbin, 1993, 1994). Teacher education programs currently include a variety of subdisciplinary courses, such as biomechanics, anatomy, exercise physiology, motor learning, history/philosophy, and psycho-social issues of movement which are thought to provide the theoretical foundation for the entire curriculum. The hypothetical relationship between these subdisciplinary courses and the other components of the PETE curriculum are illustrated in Figure 3 (Wiegand, Bulger, \& Mohr, 2004).

This emphasis on subdisciplinary research and coursework in higher education was intended to enhance the academic legitimacy of physical education (Corbin, 1993) by increasing the knowledge of prospective physical educators in subdisciplinary areas that are related to the teaching of physical education. It was theorized that the instructional effectiveness of prospective physical educators would be enhanced if connections between subdisciplinary knowledge and physical education were successfully established (Morford, 1972; O'Hanlon \& Wandzilak, 1980). There is little evidence to suggest, however, that this subdisciplinary curricular shift has significantly enhanced the instructional effectiveness of practicing physical educators (Corbin, 1993; Locke, 1990; Ross, 1981).

The closely related issue of curricular space represents a very legitimate problem for faculty members currently teaching within many PETE programs. In addition to the considerable challenge of preparing graduates who are effective novice physical educators, PETE undergraduate programs and various other academic units on campus share the responsibility of facilitating the development of liberally educated persons who are capable of making meaningful contributions to society in both their personal and professional lives. Additionally, many PETE programs provide a number of other services to students and members of the community that may potentially impact faculty workload and the availability of curricular space (e.g., academic advising, community programming, service learning, and professional development opportunities for students). The problem of curricular space is further compounded by the 
fact that increased disciplinary specialization within higher education and related advancements in research technology have lead to a proliferation of ideas and information across the past four decades within virtually every academic discipline. Based on these numerous competing interests and the continually expanding body of knowledge in physical education and its related subdisciplines, it is unlikely that PETE students will be able to master all of the concepts and skills that we would ideally like them to possess upon graduation.

The continually expanding body of subdisciplinary knowledge and limited amount of curricular space available within most PETE programs mandate some difficult decisions concerning the concepts that should be included and excluded from a curriculum. While an argument can be made regarding the importance of covering a wide breadth of content within the curriculum, the author maintains that it is important for PETE students to know more about less. In other words, students should invest their time and effort mastering designated outcomes or competencies that are most indicative of best professional practice within the field, rather than learning more numerous facts about a broader range of topics that may or may not influence the quality of physical education programming in the schools. If curricular depth rather than breadth remains the primary focus of the PETE program, then physical education teacher educators need to determine which knowledge, skills, and abilities are most critical for inclusion within the curriculum.

This argument is supported by the results of this Delphi investigation in which a very broad range of 222 potentially significant competencies in exercise science were reduced to a final list of just over 100 items (see Table 13). The final list of recommended exercise science competencies has been further summarized in Figures 4 and 5 for the purpose of this discussion. These competencies should prove valuable to PETE curriculum planners and can be used for a variety of the following purposes: (a) selecting course content, (b) evaluating curricular effectiveness in the area of exercise science, (c) aligning courses across the curriculum, (d) focusing student learning around the most critical knowledge, skills, and abilities, and (e) determining the appropriateness of various laboratory and experiential learning opportunities for students. A number of the competencies that were identified as critical may not be routinely incorporated within the PETE curriculum. Those items related to developmental exercise physiology, behavioral change, and weight management, for example, are becoming increasingly relevant for prospective physical educators who will be expected to contribute in some meaningful capacity to the fight against children's obesity and physical inactivity. It is also important to point out that both preservice and in-service physical educators should be expected to model appropriate physical activity behaviors and health-enhancing levels of physical fitness for their students (National Association for Sport and Physical Education, 2002b).

While not a primary focus of the study, the differences of opinion among Delphi panel members was considered to be an area of potential interest. In other words, were there marked differences in responses among exercise science specialists, teacher educators, and physical education teachers? One might hypothesize, for example, that exercise specialists who possess a more in depth understanding of the involved subject matter would be more inclined to rate a broader range of competencies as being both critically important and relevant than a teacher educator or physical education specialist who may be more exclusively interested in practical application. These types of differences of opinion are potentially significant because any truly integrated and well-articulated curricular model in PETE would need to address the divergent opinions that would undoubtedly exist among different faculty members.

As a follow-up analysis, the Delphi panel member responses were segmented by specialization. Group mean responses and standard deviations were calculated for those questionnaire items that were not initially rated as being critically important and relevant by the entire Delphi panel (see Table 14). The purpose of this analysis was to provide some descriptive statistical support for the differences of opinion that may exist among exercise scientists, teacher educators, and physical educators. Interestingly, there 
was limited variation among groups regarding their ratings of the non-critical exercise science competencies.

There were only three occasions when the exercise science panel members found items to be critical and the entire Delphi panel did not (Items 37, 116, and 189). The exercise scientists concluded that the knowledge of the physiological adaptations that occur at rest and during submaximal exercise was critically important and relevant. The two other groups rated that competency highly in terms of theoretical importance, but of lower pedagogical relevance. It is possible that the exercise scientists, who are likely to have a more in depth knowledge of the physiological adaptations to chronic aerobic exercise training (e.g., increased cardiac output through changes in stroke volume, increased mitochondrial density and oxygen extraction at the cellular level, little to no change in maximal heart rate), would perceive there to be more meaningful connections between the physiology of exercise and its practical application in a physical education context. For example, the decreases in resting and submaximal exercise heart rate observed in response to endurance training directly reflect an increased stroke volume of the left ventricle. One could potentially argue that it is beneficial to understand the desired physiological adaptations associated with an exercise program prior to its design, implementation, and evaluation. Additionally, the exercise scientists and physical education teachers rated the knowledge of special precautions and modifications of exercise programming for participation at altitude, different temperatures, humidity, and pollution as being critical while the teacher educators found it to be less relevant. The exercise scientists concern regarding the issues of temperature and humidity may be especially significant for prospective physical educators given the safety issues associated with a child's physiological limitations in the area of heat dissipation during exercise.

The physical education teacher educators rated 17 items as being critical in nature in contrast to the Delphi panel mean responses (Items 2, 4, 11, 18, 19, 61, 62, 79, 80, 94, 102, 121, 142, 166, 172, 193, and 221). On 11 out of 17 of those items, the physical education teachers also rated the competency as critically important and relevant. Somewhat surprisingly, the teacher educators and physical educators rated the knowledge of basic anatomical structures, biomechanical concepts, joint classification, and peripheral pulse palpation to be critical while the exercise scientists did not. The teacher educators and physical educators rated a number of competencies related to group exercise leadership as being critical (e.g., group exercise leadership techniques for working with various ages, instructional positioning to enhance participant interaction, use of verbal and nonverbal teaching cues). This result was not unexpected and it makes sense that these groups would view group exercise leadership as being highly critical to the preparation of prospective physical educators.

The physical education teachers rated 32 competencies as being critically important and pedagogically relevant on occasions when the entire panel did not (Items 4, 6, 8, 11, 18, 19, 35, 39, 40, $48,61,87,89,90,91,94,99,102,116,117,137,139,142,148,166,172,189,193,196,208,220$, and 221). The most interesting of these variations came in the area of exercise psychology and adherence. The physical education teachers were the only group that rated a number of these competencies as critical. For example, the teachers responded that it was essential for PETE students to develop competence in the following areas: (a) stages of motivational readiness, (b) strategies for encouraging initiation, adherence, and return to physical activity, (c) signs and symptoms of anxiety and depression, (d) symptoms and causal factors of test anxiety and their affect on fitness test performance, and (e) psychological factors associated with sport performance. Both the teacher educators and physical educators rated knowledge of the psychosocial determinants of physical activity highly. Exercise prescription and programming is frequently referred to as both an art and science. The science obviously relates to the application of basic training principles and the manipulation of training variables over time to elicit a specific set of physiological responses. The art of exercise prescription is much less clearly defined and concerns the practitioner's ability to motivate clients to successfully adhere to a physical activity program. This pattern of responses may indicate that the in-service physical educators are more concerned with exercise 
psychology and adherence because that is a challenge that they must address on a daily basis. It is probably an area that receives the least amount of attention in many PETE programs as well. This is unfortunate because even the most technically well-designed exercise prescription or program means very little if the students are unable to adhere to it for an extended period of time.

The physical education teachers were the only group to rate the ability to identify the major bones and identify the joints of the body as being critically important and relevant. Somewhat surprisingly, this group also regarded the following physiological competencies as critical: (a) the theories of muscle fatigue and delayed onset muscle soreness, (b) the structure of skeletal muscle fiber and the basic mechanism of contraction, (c) the characteristics of various muscle fiber types, and (d) the differences in cardiovascular response to various forms of exercise and the associated dangers. These are relatively advanced physiological concepts of clear potential theoretical importance. It is interesting, however, that the group of physical education teachers also perceived these concepts to be pedagogically relevant given the fact that muscle fiber physiology remains a fairly abstract content area and we do not typically associate school-based physical education programming with training frequencies or exercise intensity levels that produce a significant degree of muscle soreness. Additionally, children and adolescents are not generally populations at risk for dangerous cardiovascular responses to more vigorous types of physical activity.

Once again, these types of between group differences were not a focus of the study and it is impossible to generalize these results to a broader population for a number of research design considerations (e.g., small overall sample size, differences in sample size among groups within the study, selection of a non-representative panel of experts). A logical next step in this line of research would involve the circulation of the questionnaire to a more representative sample of exercise science specialists, teacher educators, and physical education teachers to get a better indication of where these types of differences of opinion may indeed occur. Based on the rather limited findings of this study, it would be desirable for PETE programs to form curriculum advisory committees including teacher educators, subdisciplinary specialists, in-service teachers, and program alumni who may have different perspectives on the importance and relevance of various proposed instructional competencies.

Ideally, these theoretical and applied exercise science competencies that are ultimately judged to be most critical would be incorporated in an exercise physiology course designed specifically for PETE majors. A more homogeneous course enrollment would allow the instructor to select only the most important and highly relevant competencies for prospective physical educators, whose needs and interests vary greatly from other pre-professional students preparing for careers in athletic training, exercise science, physical therapy, chiropractic, and so forth. A student planning for a career in cardiac rehabilitation, for example, would need a much more complete understanding of cardiovascular physiology, pathology of coronary artery disease, pre-activity health screening, pharmacological interventions in disease management, relationship between nutrition and disease, and developmental considerations associated with exercise in older adults and various other special populations (e.g., metabolic, cardiovascular, pulmonary, and musculoskeletal disease). As suggested by the results of this study, a prospective physical educator requires a very basic understanding of anatomy, biomechanics, exercise physiology and human nutrition, with a much greater emphasis placed on pediatric exercise considerations. Comparatively, students preparing to teach physical education need much more thorough training in the areas of behavior change, field-based physical fitness testing, emergency preparedness, and exercise programming in group settings. These issues of practical application are not typically addressed in introductory exercise physiology courses, which focus more directly on the body's physiological response to physical activity within the cells, tissues, organs, and systems responsible for human movement. 
In reality, many departments lack adequate resources and expertise to offer multiple sections of introductory exercise physiology courses for students with divergent academic backgrounds and professional interests. If this is the case, it is critical that PETE curriculum developers include a course(s) later in the plan of study that specifically addresses the numerous issues associated with effective fitness education within school-based physical education programs. This course would actively reinforce and build upon the critical theoretical concepts learned during an introductory exercise physiology course by demonstrating how they are actually applied in a practical physical education setting. The primary focus of this teaching of fitness education course would include the following objectives: (a) Assessing children's physical activity and fitness needs (e.g., attitudinal surveys, behavioral questionnaires, fitness test protocols); (b) designing physical activity programs and leading group exercise sessions; (c) establishing a safe and supportive exercise environment; and (d) using a variety of behavioral strategies for promoting exercise adherence and promoting lifestyle activity (e.g., goal setting, behavioral contracts, social support, reinforcement). A lab or service learning requirement involving the administration of a field-based fitness education program for children or adolescents would be a particularly helpful experience for prospective physical educators enrolled in this type of alternative exercise science course. See Appendix D for an example of a well-designed, alternative fitness education course that has been implemented within a PETE curriculum.

\section{Exercise Science Instructional Methods}

Within the PETE program, courses in the foundational sciences are frequently taught by faculty members whose graduate training for the academic profession focuses almost exclusively on disciplinary knowledge and research proficiency. In his review of current issues confronting those teaching within higher education, Kennedy (1999) proposed that this approach to graduate study may negatively detract from the university's core responsibility of educating the public:

Too often, little attention is given to the teaching responsibilities that doctoral students will have later, as they are pressed to finish their dissertations or to support their mentors' research programs. Faculty members-in-training receive little or no preparation for the range of personal and professional challenges they will face as members of the professoriate. The vast majority of academic doctorates are produced in fewer than one hundred 'research universities,' but 90 percent of them will teach in several thousand institutions that are quite different from the places in which they were trained. They arrive on the job with little guidance about how to make the transition from expert learner to novice teacher, or even about what is expected of them as professionals. (p. 30)

The promotion and tenure process at most universities is also designed to reward productivity in research as a more traditional expression of scholarly engagement, rather than the development of effective and innovative instructional practices for use in the classroom (Huber, 2002). It may be unrealistic for institutions to place too many demands on faculty regarding the scholarship of teaching because "at most four-year institutions, research distinction and productivity continue to be seen as more important than teaching excellence, and mobility both within and across institutions depends largely on scholarly reputation" (Colby, Ehrlich, Beaumont, \& Stephens, 2003, p.35). While there is some evidence that this emphasis may be shifting on college campuses (Kennedy, 1999), there are a number of related characteristics of academic life that potentially interfere with curricular articulation and instructional effectiveness: (a) The shift in faculty attention and loyalty away from educational pursuits and the university to academic disciplines and departments; (b) the lack of commensurate awards for time and effort spent on creative and innovative instructional approaches; (c) the heavy course loads assigned to faculty at many regional and comprehensive universities; (d) the reliance on student credit hours alone to determine teaching load without consideration for the nature of the involved pedagogies and the associated time constraints; (e) the lack of attention commonly paid to teaching early in the careers of 
doctoral students and junior faculty members; and (f) the considerable emphasis placed on faculty autonomy at most institutions "makes it very difficult to structure undergraduate education to embody cross-cutting learning goals or to ensure that courses connect with and build on each other systematically, supporting cumulative advancement in the development of complex intellectual capacities" (Colby et al., 2003, p. 34).

If the required degree of curricular articulation and integration is to be achieved, physical education teacher educators and exercise science specialists need to be concerned with how content is organized and delivered across the curriculum. If theoretically important and pedagogically relevant subdisciplinary content is to be included within the PETE curriculum, it is logical to expect that the review and practical application of all this information will occur repeatedly throughout the remainder of the curriculum. The infusion of curricular strands represents an extremely important characteristic of any curriculum intending to optimize its effectiveness (Bulger, Mohr, Carson et al., 2000; Bulger et al., 2001). Bulger et al. (2001) described curricular strands as "those critical themes or perspectives that are typically introduced within the foundational semesters of the undergraduate curriculum and systematically revisited in a wide variety of instructional contexts" (pp. 404). If the subdisciplinary concept is deemed important and relevant enough to be included in the PETE curriculum, then it should be systematically revisited or stranded throughout. Subdisciplinary knowledge areas not infused as strands within a PETE curriculum are either not pedagogically relevant enough to be included in the first place or are pedagogically relevant but not being fully optimized within the curriculum.

Given the reality of the numerous individual, social, and environmental barriers to instructional effectiveness within higher education, it is imperative that PETE faculty members engage in an ongoing discussion with their colleagues in the various subdisciplines and physical educators working in the field to continually refine the instructional approaches used in teacher preparation. Unfortunately, many of the relevant subdisciplinary concepts that may contribute to a prospective physical educator's ability to initiate meaningful behavior change in a child's physical activity patterns are overlooked by the student because teacher educators fail to specifically account for this theory to practice transition in their instruction. The results of this study demonstrate that improved exercise science content instruction in PETE should involve a variety of instructional methods that are intended: (a) to promote higher levels of student engagement; (b) to exemplify the pedagogical relevance of certain critical subdisciplinary concepts; (c) to serve as the foundational basis for informational strands which are infused throughout the entire PETE curriculum; and (d) to enhance the prospective physical education teacher's willingness and ability to apply these concepts in a school-based setting.

In many PETE programs, subdisciplinary courses continue to emphasize the delivery of foundational knowledge in a lecture and discussion format which may ultimately inhibit the student's active engagement in the teaching-learning process. While the results of this study do support the continued use of teacher-directed forms of instruction in exercise science courses, these traditional instructional approaches may tend to reinforce the passive routines of students rather than engaging them in the types of active learning activities that will enable them to think critically and reflectively about teaching physical education (Tinning, 1988).

Accordingly, exercise science content specialists should be encouraged to experiment with the types of alternative pedagogical approaches proposed in this study through the provision of faculty release time, continued mentorship regarding teaching effectiveness, and greater recognition of the scholarship of teaching by university administrators and department personnel committees. It is worth noting that few, if any, of the instructional methods recommended by this panel of experts are particularly novel or innovative. All of the proposed instructional methods have been previously recommended in the PETE literature, along with a number of other fundamentally sound pedagogical approaches. In short, we maintain a very thorough understanding of what effective teaching involves, but it remains highly 
unlikely that the overall quality of teaching within subdisciplinary courses will be heavily influenced by that body of knowledge if the involved faculty members are not provided with access to adequate teacher training as doctoral students and resources during their first few years of employment (e.g., access to current instructional technologies, professional development opportunities related to teaching, balanced teaching/service/research/advising loads and expectations, rewards for innovative teaching practices during annual performance reviews and the tenure/promotion process). 


\section{REFERENCES}

American Academy of Pediatrics. (1987). Physical fitness and the schools. Pediatrics, 80, 449-450.

American Academy of Pediatrics. (1990). Strength training, weight and power lifting, and body building by children and adolescents. Pediatrics, 86, 801-803.

American Alliance for Health, Physical Education, Recreation and Dance. (1999). Physical education for lifelong fitness: The Physical Best teacher's guide. Champaign, IL: Human Kinetics.

American College of Sports Medicine. (1988). ACSM opinion statement on physical fitness in children and youth. Medicine and Science in Sports and Exercise, 20, 422-423.

American College of Sports Medicine (1990). The recommended quantity and quality of exercise for developing and maintaining cardiorespiratory and muscular fitness in healthy adults. Medicine and Science in Sports and Exercise, 22, 265-274.

American College of Sports Medicine. (1994). Exercise for patients with coronary artery disease. Medicine and Science in Sports and Exercise, 26, i-v.

American College of Sports Medicine. (1995). Osteoporosis and exercise. Medicine and Science in Sports and Exercise, 27, i-vii.

American College of Sports Medicine. (1998a). Exercise and physical activity for older adults. Medicine and Science in Sports and Exercise, 30, 992-1008.

American College of Sports Medicine. (1998b). The recommended quantity and quality of exercise for developing and maintaining cardiorespiratory and muscular fitness, and flexibility in healthy adults [Electronic version]. Medicine and Science in Sports and Exercise, 30, 975-991.

American College of Sports Medicine. (2000). ACSM's guidelines for exercise testing and prescription $\left(6^{\text {th }}\right.$ ed.). Baltimore, MD: Lippincott Williams \& Wilkins.

American College of Sports Medicine. (2001). Appropriate intervention strategies for weight loss and prevention of weight regain for adults. Medicine and Science in Sports and Exercise, 33, 2145-2156.

American College of Sports Medicine. (2002). Progression models in resistance training for healthy adults. Medicine and Science in Sports and Exercise, 34, 364-380.

American Heart Association. (1996a). Statement on exercise: Benefits and recommendations for physical activity programs for all Americans [Electronic version]. Circulation, 94, 857-862.

American Heart Association. (1996b). Understanding obesity in youth [Electronic version]. Circulation, 94, 3383-3387.

American Orthopedic Society for Sports Medicine. (1988). Proceedings of the conference on strength training and the prepubescent. Chicago, IL: AOSSM publishing.

Bahneman, C.P. (1996). An analysis of the undergraduate physical education teacher certification requirements within institutions which offer a doctoral degree in physical education. Physical Educator, 53, 198-202. 
Bain, L.L. (1981). Basic Stuff series. Journal of Physical Education and Recreation, 52(2), 33-34.

Bain, L.L., \& Poindexter, H.B.W. (1981). Applying disciplinary knowledge in professional preparation. Journal of Physical Education and Recreation, 52(2), 40-41.

Baldwin-Morgan, A.A. (1993). The impact of expert system audit tools on auditing firms in the year 2001: A Delphi investigation [Electronic version]. Journal of Information Systems, 7(1), 16-33.

Barnett, B.E., \& Merriman, W.J. (1994). Knowledge of physical fitness in prospective physical education teachers [Electronic version]. Physical Educator, 51, 74-77.

Bar-Or, O. (1999). Health benefits of physical activity during childhood and adolescence. In C.B. Corbin \& R.P. Pangrazi (Eds.), Toward a better understanding of physical fitness and activity (pp. 163-167). Scottsdale, AZ: Holcomb Hathaway Publishers.

Bar-Or, O. (2000). Juvenile obesity, physical activity, and lifestyle changes: Cornerstones of prevention and management. The Physician and Sports Medicine, 28(11), 51-58.

Biddle, S. (1995). Exercise and psychosocial health. Research Quarterly for Exercise and Sport, 66, 292297.

Blair, S.N., Clark, D.B., \& Cureton, K.J. (1989). Exercise and fitness in childhood: Implications for a lifetime of health. In C.V. Gisolfi \& D.L. Lamb (Eds.), Perspectives in exercise science and sports medicine, Vol. 2.Youth, exercise and sport (pp. 401-430). Indianapolis, IN: Benchmark Press.

Bouchard, C., \& Després, J-P. (1995). Physical activity and health: Artherosclerotic, metabolic, and hypertensive diseases. Research Quarterly for Exercise and Sport, 66, 268-275.

Brooks, K.W. (1979). Delphi technique: Expanding applications. North Central Association Quarterly, 53, 377-385.

Brophy, J.E., \& Good, T.L. (1986). Teacher behavior and student achievement. In M.C. Wittrock (Ed.), Handbook of research on teaching ( ${ }^{\text {rd }}$ ed.) (pp. 328-375). New York, NY: Macmillan.

Bulger, S.M., Mohr, D.J, Carson, L.M, \& Wiegand, R.L. (2001). Infusing health-related physical fitness in physical education teacher education. Quest, 53, 403-417.

Bulger, S.M., Mohr, D.J., \& Wiegand, R.L. (2003). Factors effecting student learning in traditional, theory based versus alternative, pedagogical content knowledge based exercise physiology courses: An explanatory study. Research Quarterly for Exercise and Sport, 73 (Suppl. 1), A-38.

Bulger, S.M., Mohr, D.J., Carson, L.M., Robert, D.L., \& Wiegand, R.L. (2000). Preparing prospective physical educators in exercise physiology. Quest, 52, 166-185.

Bulger, S.M., Mohr, D.J., Graves, M.A., \& Wiegand, R.L. (1999, October). Preparing physical education teacher educators to be subdisciplinary specialists. Paper presented at the National Teacher Education Conference of the National Association for Sport and Physical Education (NASPE), Bloomingdale, IL. 
Bulger, S.M., Mohr, D.J., Graves, M.A., \& Wiegand, R.L. (2000). The effectiveness of traditional, theory based versus alternative, pedagogical content knowledge based exercise physiology courses at delivering exercise physiology content knowledge to physical education teacher education students. Research Quarterly for Exercise and Sport, 71 (Suppl. 1), A-66.

Casperson, C.J., Nixon, P.A., \& Durant, R.H. (1998). Physical activity epidemiology applied to children and adolescents. Exercise and Sport Science Reviews, 26, 341-403.

Centers for Disease Control and Prevention \& American College of Sports Medicine. (1995). Physical activity and public health: A recommendation from the Centers for Disease Control and Prevention and the American College of Sports Medicine. Journal of the American Medical Association, 273, 402-407.

Chiappetta, E.L., \& Fillman, D.A. (1998). Clarifying the place of essential topics and unifying principles in high school biology [Electronic version]. School Science and Mathematics, 98(1), 12-19.

Cicek, S., \& Demirhan, G. (2001). Validating teacher competencies for Turkish physical education teachers: A national study using focus Delphi Method. International Journal of Physical Education, 38, 34-42.

Clayton, M.J. (1997). Delphi: A technique to harness expert opinion for critical decision-making tasks in education [Electronic version]. Educational Psychology, 17, 373-387.

Colby, A., Ehrlich, T., Beaumont, E., \& Stephens, J. (2003). Educating citizens: Preparing America's undergraduates for lives of moral and civic responsibility. San Francisco, CA: Jossey-Bass.

Collier, C.S., \& O'Sullivan, M. (1997). Case method in physical education higher education: A pedagogy of change? Quest, 49, 198-213.

Cooper, D.A. (1982). Development of an inservice manual and instructor's guide for adapted physical education (Doctoral dissertation, University of Georgia, 1982). Dissertation Abstracts International, 43, 394.

Corbin, C.B. (1993). Clues from dinosaurs, mules, and the bull snake: Our field in the $21^{\text {st }}$ century. Quest, 45, 546-556.

Corbin, C.B. (1994). Current status of HPER in the United States. In P. Duffy \& L. Dugdale (Eds.), HPER - Moving toward the $21^{\text {st }}$ century (pp. 313-325). Champaign, IL: Human Kinetics.

Corbin, C.B., \& Pangrazi, R.P. (1994). Toward an understanding of appropriate physical activity levels for youth. President's Council on Physical Fitness and Sports Physical Activity and Fitness Research Digest, 1(8), 1-8.

Corbin, C.B., \& Pangrazi, R.P. (1996a). How much physical activity is enough? Journal of Physical Education, Recreation and Dance, 67(4), 33-37.

Corbin, C.B., \& Pangrazi, R.P. (1996b). What you need to know about the Surgeon General's Report on Physical Activity and Health. President's Council on Physical Fitness and Sports Physical Activity and Fitness Research Digest, 2(6), 1-8. 
Corbin, C.B., \& Pangrazi, R.P. (Eds.). (1999). Toward a better understanding of physical fitness and activity. Scottsdale, AZ: Holcomb Hathaway Publishers.

Cottone, J.L. (1996). The use of evaluation in the professional preparation of athletic trainers to assess clinical performance and determine clinical competency: A Delphi study (Doctoral dissertation, Syracuse University, 1996). Dissertation Abstracts International, 57, 2410.

Dalkey, N.C. (1967). Delphi. Santa Monica, CA: The RAND Corporation (P-3704).

Delbecq, A.L., Van de Ven, A., \& Gustafson, D. (1975). Group techniques for program planning: A guide to Nominal Group and Delphi processes. Glenview, IL: Scott, Foresman, and Company.

Dodds, P. (1987, April). Training physical education teaching majors: Connections between exercise science and teaching practice. Paper presented at the meeting of the American Alliance for Health, Physical Education, Recreation and Dance, Las Vegas, NV.

Dollman, J. (2003). A problem-based approach to teaching exercise physiology gender differences in athletic performance. Journal of Physical Education, Recreation and Dance, 74(9), 37-42.

Durant, R.H., Baranowski, T., Davis, H., Rhodes, T., Thompson, W.O., Greaves, K.A., \& Puhl, J. (1993). Reliability and variability of indicators of heart rate monitoring of children. Medicine and Science in Sports and Exercise, 25, 389-395.

Durant, R.H., Baranowski, T., Davis, H., Thompson, W.O., Puhl, J., Greaves, K.A., \& Rhodes, T. (1992). Reliability and variability of heart rate monitoring in 3-, 4-, or 5-year-old children. Medicine and Science in Sports and Exercise, 24, 265-271.

Erikssen, G. (2001). Physical fitness and changes in mortality: The survival of the fittest. Sports Medicine, 31, 571-576.

Estes, S. (1994). Knowledge and kinesiology. Quest, 46, 392-409.

Feingold, R.S. (1994). Making connections: An agenda for the future. Quest, 46, 356-367.

Fernandez-Balboa, J.M., Barrett, K., Solomon, M., \& Silverman, S. (1996). Perspectives on content knowledge in physical education. Journal of Physical Education, Recreation and Dance, 67(9), 5457,61 .

Fleming, J.L., \& Monda-Amaya, L.E. (2001). Process variables critical for team effectiveness: A Delphi study of wraparound team members. Remedial and Special Education, 22, 158-171.

Flippo, R.F. (1998). Points of agreement: A display of professional unity in our field [Electronic version]. Reading Teacher, 52(1), 30-41.

Franks, B.D. (1999). Personalizing physical activity prescription. In C.B. Corbin \& R.P. Pangrazi (Eds.), Toward a better understanding of physical fitness and activity (pp. 19-24). Scottsdale, AZ: Holcomb Hathaway Publishers.

Fulton, J.E., McGuire, M.T., Casperson, C.J., \& Dietz, W.H. (2001). Interventions for weight loss and weight gain prevention among youth. Sports Medicine, 31, 153-165. 
Gardner, J.N., \& Van der Veer, G. (Eds.) (1997). The senior experience: Facilitating integration, reflection, closure, and transition. San Francisco, CA: Jossey-Bass.

Gillespie, A.M., \& Lowe, J.B. (1995). Use of the Delphi process for initial investigation of community education on bowel cancer: A study [Electronic version]. Journal of Wellness Perspectives, 12(1), $35-42$.

Gilliam, T.B., Freedson, P.S., Geenen, D.L., \& Shahraray, B. (1981). Physical activity patterns determined by heart rate monitoring in 6-7 year-old children. Medicine and Science in Sports and Exercise, 13, 65-67.

Goldschmidt, P. (1975). 'Scientific inquiry or political critique? Remarks on Delphi assessment, expert opinion, forecasting and group process by H. Sackman.' Technological Forecasting and Social Change, 7, 195-213.

Good, J.J. (1998). Recommendations for change in physical education: A survey of selected physical education professionals (Doctoral dissertation, The University of New Mexico, 1998). Dissertation Abstracts International, 59, 2417.

Gordon, T.J. (1992). The methods of futures research [Electronic version]. Annals of the American Academy of Political and Social Science, 522, 25-36.

Gortmaker, S.L., Dietz, W.H., Sobol, A.M., \& Wehler, C.A. (1987). Increasing pediatric obesity in the United States. American Journal of Diseases in Children, 141, 535-540.

Greendorfer, S.L. (1987a). The role of sport studies in sport pedagogy: Who needs it? In G. Barrette, R.S. Feingold, C.R. Rees, \& M. Pierone (Eds.), Myths, models, and methodologies in sport pedagogy (pp. 47-50). Champaign, IL: Human Kinetics.

Greendorfer, S.L. (1987b). Specialization, fragmentation, integration, discipline, profession: What is the real issue? Quest, 39, 56-64.

Griffin, L., Dodds, P., \& Rovegno, I. (1996). Pedagogical content knowledge for teachers: Integrate everything you know to help students learn. Journal of Physical Education, Recreation and Dance, 67(9), 58-61.

Haskell, W.L. (1999). Physical activity in prevention and management of coronary heart disease. In C.B. Corbin \& R.P. Pangrazi (Eds.), Toward a better understanding of physical fitness and activity (pp. 71-79). Scottsdale, AZ: Holcomb Hathaway Publishers.

Hellison, D. (1991). The whole person in physical education scholarship: Toward integration. Quest, 43, 307-318.

Henry, F.M. (1964). Physical education: An academic discipline. Journal of Health, Physical Education and Recreation, 35(7), 32-33, 69.

Hill, G.M., \& Randle, K. (1992). Fitness strategies in high school physical education programs [Electronic version]. Physical Educator, 49, 195-205.

Hoffman, S.J. (1985). Specialization + fragmentation = extermination. Journal of Physical Education, Recreation and Dance, 56(6), 19-22. 
Hopkins, P.W. (1999). Leadership responses to change in $21^{\text {st }}$ century California State University physical education/kinesiology departments (Doctoral dissertation, University of San Diego, 1999). Dissertation Abstracts International, 59, 4019.

Housner, L.D. (1990). Selecting master teachers: Evidence from process-product research. Journal of Teaching in Physical Education, 9, 201-226.

Housner, L.D. (1991). Teacher cognition. In T. Martenik (Ed.) Psycho-social dynamics of teaching physical education (pp. 35-58). Dubuque, IA: William C. Brown Publishing.

Housner, L.D., \& French, K.E. (1994). Future directions for research on expertise in learning, performance, and instruction in sport and physical activity. Quest, 46, 241-246.

Huber, M.T. (2002). Faculty evaluation and the development of academic careers. In C. Colbeck (Ed.) Evaluating faculty performance (New Directions for Institutional Research No. 114). San Francisco: Jossey-Bass.

Jacobs, J.M. (1996). Essential assessment criteria for physical education teacher education programs: A Delphi study (Doctoral dissertation, West Virginia University, 1996). Dissertation Abstracts International, 57, 2938.

Janz, K.F., Golden, J.C., Hansen, J.R., \& Mahoney, L.T. (1992). Heart rate monitoring of physical activity in children and adolescents: The Muscatine study. Pediatrics, 89, 256-261.

Jeffery, G., \& Hache, G. (1995). A group-based Delphi application: Defining rural career counseling needs. Measurement and Evaluation in Counseling and Development, 28(1), 45-61.

Johnson, C.C., Nicklas, T.A., Arbeit, M.L., Harsha, D.W., Mott, D.S., Hunter, S.M., Wattigney, W., \& Berenson, G.S. (1991). Cardiovascular intervention for high-risk families: The Heart Smart Program. Southern Medical Journal, 84, 1305-1312.

Johnson, J.R. (1992). A survey of Division II athletic and physical education fiscal trends for the next twenty years (Doctoral dissertation, West Virginia University, 1992). Dissertation Abstracts International, 54, 1254.

Karper, W.B. (1997). Exercise physiology course content - Is it practical for most teachers? Journal of Physical Education, Recreation and Dance, 68(2), 46-48.

Kazdin, A.E. (1982). Single-case research designs. New York, NY: Oxford University Press.

Kelley, E.J., \& Lindsay, C.A. (1977). Knowledge obsolescence in physical educators. Research Quarterly, 48, 463-474.

Kelley, E.J., \& Lindsay, C.A. (1980). A comparison of knowledge obsolescence of graduating seniors and practitioners in the field of physical education. Research Quarterly for Exercise and Sport, 51, 636644.

Kennedy, D. (1999). Academic duty. Cambridge, MA: Harvard University Press. 
Kneer, M. (Ed.). (1981). Basic Stuff Series. Reston, VA: American Alliance for Health, Physical Education, Recreation and Dance Publications.

Knudson, D. (2003). An integrated approach to the introductory biomechanics course [Electronic version]. Physical Educator, 60, 122-134.

Korthagen, F.A., \& Kessels, P.A.M. (1999). Linking theory and practice: Changing the pedagogy of teacher education. Educational Researcher, 28(4), 4-17.

Kuntzleman, C.T., \& Reiff, G.G. (1992). The decline in American children's fitness level. Research Quarterly for Exercise and Sport, 63, 107-111.

LaPlante, M.J. (1973). Evaluation of a selected list of purposes for physical education using a modified Delphi technique. Unpublished doctoral dissertation, University of Wisconsin, Madison.

LaPlante, M.J., \& Jewett, A.E. (1987). Content validation for the purpose dimension. Journal of Teaching in Physical Education, 6, 214-223.

Lawson, H.A. (1990). Sport pedagogy research: From information-gathering to useful knowledge. Journal of Teaching in Physical Education, 10, 1-20.

Lawson, H.A. (1991). Specialization and fragmentation among faculty as endemic features of academic life. Quest, 43, 280-295.

Lee, I-M. (1995). Exercise and physical health: Cancer and immune function. Research Quarterly for Exercise and Sport, 66, 286-291.

Lee, I-M. (1999). Physical activity and cancer. In C.B. Corbin \& R.P. Pangrazi (Eds.), Toward a better understanding of physical fitness and activity (pp. 81-87). Scottsdale, AZ: Holcomb Hathaway Publishers.

Lidstone, J.E., \& Feingold, R.S. (1991). Introduction - The case for integration and collaboration, reprise. Quest, 43, 241-246.

Linger, B.T. (1998). What primary care physicians need to know about attention-deficit hyperactivity disorder: A Delphi of experts and a content analysis. Unpublished doctoral dissertation, West Virginia University, Morgantown.

Livingston, L.A. (1996). Re-defining the role of physical activity courses in the preparation of physical education teaching professionals. Physical Educator, 53, 114-121.

Locke, L.F. (1990). Why motor learning is ignored: A case of ducks, naughty theories, and unrequited love. Quest, 42, 134-142.

Loughery, T.J. (1985). Basic Stuff in a professional preparation program. Journal of Physical Education, Recreation and Dance, 56(8), 11-13.

MacConnie, S.E., Gilliam, T.B., Geenen, D.L., \& Pels, A.E. (1982). Daily physical activity patterns of prepubertal children involved in a vigorous exercise program. International Journal of Sports Medicine, 3, 202-207. 
Martin, R.H. (1986). An investigation of the future of doctorate study in physical education: A Delphi study (Doctoral dissertation, University of Southern Carolina, 1986). Dissertation Abstracts International, 48, 77.

McBride, R.E. (1999). If you structure it, they will learn: Critical thinking in physical education classes [electronic version]. Clearing House, 72, 217-220.

McKenzie, T.L. (1999). School health-related physical activity programs: What do the data say? Journal of Physical Education Recreation, Recreation and Dance, 70(1), 16-19.

McKenzie, T.L., \& Alcaraz, J.E. (1994). Children's enjoyment of activity units in a health-related physical education curriculum: SPARK [Abstract]. Research Quarterly for Exercise and Sport, 65, A-79.

McKenzie, T.L., \& Sallis, J.F. (1996). Physical activity, fitness, and health-related physical education. In S.J. Silverman \& C.D. Ennis (Eds.), Student learning in physical education: Applying research to enhance instruction (pp. 223-246). Champaign, IL: Human Kinetics.

McKenzie, T.L., \& Strikmiller, P.K. (1994). CATCH: Physical activity process evaluation in a multicenter trial [Electronic version]. Health Education Quarterly, 21(Suppl. 2), S73-S90.

McKenzie, T.L., Nader, P.R., Strikmiller, P.K., Yang, M., Stone, E.J., Perry, C.L., Taylor, W.C., Epping, J.N., Feldman, H.A., Luepker, R.V., \& Kelder, S.H. (1996). School physical education: Effect of the Child and Adolescent Trial for Cardiovascular Health. Preventive Medicine, 25, 423-431.

McKenzie, T.L., Sallis, J.F., Faucette, F.N., \& Kolody, B. (1997). Long-term effects of a physical education curriculum and staff development program: SPARK. Research Quarterly for Exercise and Sport, 68, 280-291.

Metzler, M.W. (1994). Scholarship reconsidered for the professoriate of 2010. Quest, 46, 440-455.

Miller, M.G., \& Housner, H.L. (1998). A survey of health-related physical fitness knowledge among preservice and inservice physical educators [Electronic version]. Physical Educator, 55, 176-187.

Mohr, D.J., Bulger, S.M., Graves, M., \& Wiegand, R.L. (1999, October). A systematic strategy for assessing PETE programs. Paper presented at the National Teacher Education Conference of the National Association for Sport and Physical Education, Bloomingdale, IL.

Mood, D. (1971). Where do physical education majors learn about fitness? Physical Educator, 28, 75-76.

Morford, W.R. (1972). Toward a profession, not a craft. Quest, 18, 88-93.

Morine-Dershimer, G. (1989). Preservice teachers' conceptions of content and pedagogy: Measuring growth in reflective, pedagogical decision-making. Journal of Teacher Education, 40, 46-52.

Murry, J.W. Jr., \& Hammons, J.O. (1995). Delphi: A versatile methodology for conducting qualitative research. The Review of Higher Education, 18, 423-436.

Nader, P.R., Stone, E.J., Lytle, L.A., Perry, C.L., Osganian, S.K., Kelder, S., Webber, L.S., Elder, J.P., Montgomery, D., Feldman, H.A., Wu, M., Johnson, C., Parcel, G.S., \& Luepker, R.V. (1999). Three- 
year maintenance of improved diet and physical activity: The CATCH cohort. Child and Adolescent Trial for Cardiovascular Health. Archive of Pediatric Adolescent Medicine, 153, 695-704.

National Association for Sport and Physical Education. (1998a). Guidelines for teacher preparation in physical education: NASPE/NCATE guidelines ( $4^{\text {th }}$ ed.). Reston, VA: NASPE Publications.

National Association for Sport and Physical Education. (1998b). Physical activity for children: A statement of guidelines. Reston, VA: NASPE Publications.

National Association for Sport and Physical Education. (1998c). Shape of the nation report: A survey of state physical education requirements. Reston, VA: NASPE Publications.

National Association for Sport and Physical Education. (2002a). Physical activity guidelines for infants and toddlers. Reston, VA: NASPE Publications.

National Association for Sport and Physical Education. (2002b). Physical activity and fitness recommendations for physical activity professionals. Reston, VA: NASPE Publications.

National Association for Sport and Physical Education. (2004a). Moving into the future: National standards for physical education ( $2^{\text {nd }}$ ed.). Reston, VA: NASPE Publications.

National Association for Sport and Physical Education. (2004b). Physical activity for children: A statement of guidelines for children ages 5-12 (2 ${ }^{\text {nd }}$ ed.). Reston, VA: NASPE Publications.

National Strength and Conditioning Association. (1996). Youth resistance training: Position statement paper and literature review. Strength and Conditioning Journal, 18(6), 62-75.

National Strength and Conditioning Association. (2001). Health aspects of resistance exercise and training. Strength and Conditioning Journal, 23(6), 9-23.

Newell, K.M. (1990a). Kinesiology: The label for the study of physical activity in higher education. Quest, 42, 269-278.

Newell, K.M. (1990b). Physical activity, knowledge types, and degree programs. Quest, 42, 243-268.

Newell, K.M. (1990c). Physical education in higher education: Chaos out of order. Quest, 42, 227-242.

O'Hanlon, J., \& Wandzilak, T. (1980). Physical education: A professional field. Quest, 32, 52-59.

Pangrazi, R.P., \& Corbin, C.B. (1990). Age as a factor relating to physical fitness test performance. Research Quarterly for Exercise and Sport, 61, 410-414.

Pangrazi, R.P., \& Corbin, C.B. (1993). Physical fitness: Questions teachers ask. Journal of Physical Education, Recreation and Dance, 64(7), 14-19.

Pangrazi, R.P., Corbin, C.B., \& Welk, G.J. (1996). Physical activity for children and youth. Journal of Physical Education, Recreation and Dance, 67(4), 38-43.

Parentè, F.J., \& Anderson-Parentè, J. (1987). Delphi inquiry systems. In G. Wright \& P. Ayton (Eds.), Judgmental forecasting (pp. 129-156). New York, NY: John Wiley \& Sons. 
Pate, R.R., \& Hohn, R.C. (1994). A contemporary mission for physical education. In R.R. Pate \& R.C. Hohn (Eds.), Health and fitness through physical education (pp. 1-8). Champaign, IL: Human Kinetics.

Perry, C.L., \& Sellers, D.E. (1997). The Child and Adolescent Trial for Cardiovascular Health (CATCH): Intervention, implementation, and feasibility study for elementary schools in the United States [Electronic version]. Health Education and Behavior, 24, 716-736.

Perry, C.L., Stone, E.J., Parcel, G.S., Ellison, R.C., Nader, P.R., \& Webber, L.S. (1990). School-based cardiovascular health promotion: The Child and Adolescent Trial for Cardiovascular Health (CATCH). Journal of School Health, 60, 406-413.

Plimpton, C.E. (1987). Childhood obesity a concern for the physical educator. Journal of Physical Education, Recreation and Dance, 58, 24-26.

Plowman, S.A. (1999). Physical fitness and healthy low back function. In C.B. Corbin \& R.P. Pangrazi (Eds.), Toward a better understanding of physical fitness and activity (pp. 107-115). Scottsdale, AZ: Holcomb Hathaway Publishers.

Pollard, R., \& Tomlin, M.E. (1995). The use of expert teachers to improve education [Electronic version]. Education, 116(1), 3-9.

Pollock, M.L., \& Vincent, K.R. (1999). Resistance training for health. In C.B. Corbin \& R.P. Pangrazi (Eds.), Toward a better understanding of physical fitness and activity (pp. 119-125). Scottsdale, AZ: Holcomb Hathaway Publishers.

Rickard, G.L., \& Boswell, B. (1991). A framework for preservice instructional development._Journal of Physical Education, Recreation and Dance, 62(5), 31-32, 75-76.

Robertson, L.D., \& Heyden, S.M. (1985). Integrating the subdisciplines - A culminating experience for undergraduate physical educators. Physical Educator, 42, 65-71.

Rosenshine, B., \& Stevens, R. (1986). Teaching functions. In M.C. Wittrock (Ed.), Handbook of research on teaching ( $3^{\text {rd }}$ ed.) (pp. 376-391). New York, NY: Macmillan.

Ross, J.G., \& Gilbert, G.G. (1985). The national children and youth fitness study: A summary of findings. Journal of Physical Education, Recreation and Dance, 56(1), 45-50.

Ross, J.G., \& Pate, R.R. (1987). The national children and youth fitness study II: A summary of findings. Journal of Physical Education, Recreation and Dance, 58(9), 51-56.

Ross, S. (1981). The epistemic geography of physical education: Addressing the problem of theory and practice. Quest, 33, 42-54.

Rotondi, A., \& Gustafson, D. (1996). Theoretical, methodological, and practical issues arising out of the Delphi Method. In M. Adler \& E. Ziglio (Eds.), Gazing into the oracle: The Delphi Method and its application to social policy and public health (pp. 34-55). Bristol, PA: Jessica Kingsley Publishers.

Rovegno, I. (1993). The development of curricular knowledge: A case of problematic pedagogical content knowledge during advanced knowledge acquisition. Research Quarterly for Exercise and Sport, 64, 56-68. 
Rowland, T.W. (1996). Developmental exercise physiology. Champaign, IL: Human Kinetics.

Sackman, H. (1974). Delphi critique: Expert opinion, forecasting and group process. Lexington, MA: D.C. Heath.

Sahakian, C.E. (1997). The Delphi Method. Skokie, IL: The Corporate Partnering Institute.

Sallis, J.F., \& McKenzie, T.L. (1997). The effects of a 2-year physical education program (SPARK) on physical activity and fitness in elementary school students [Electronic version]. American Journal of Public Health, 87, 1328-1335.

Sallis, J.F., \& Patrick, K. (1994). Physical activity guidelines for adolescents: Consensus statement. Pediatric Exercise Science, 6, 302-314.

Sallis, J.F., McKenzie, T.L., Bohdan, K., Lewis, M., Marshall, S., \& Rosengard, P. (1999). Effects of health-related physical education of academic achievement: Project SPARK. Research Quarterly for Exercise and Sport, 70, 127-134.

Sallis, J.F., Patrick, K., \& Long, B.J. (1994). An overview of the International Consensus Conference on Physical Activity Guidelines for Adolescents. Pediatric Exercise Science, 6, 299-301.

Sallis, J.F., Prochaska, J.J., \& Taylor, W.C. (2000). A review of correlates of physical activity of children and adolescents. Medicine and Science in Sports and Exercise, 32, 963-975.

Schaeffer, S.C. (1998). The development of a knowledge assessment instrument regarding the abuse of selected ergogenic aids (Doctoral dissertation, Texas A\&M University, 1998). Dissertation Abstracts International, 59, 2875.

Shaw, J.M., \& Snow, C. (1999). Osteoporosis and physical activity. In C.B. Corbin \& R.P. Pangrazi (Eds.), Toward a better understanding of physical fitness and activity (pp. 99-105). Scottsdale, AZ: Holcomb Hathaway Publishers.

Shea, E.J. (1985). Research synthesis: A coherent view of physical education. Journal of Physical Education, Recreation and Dance, 56(8), 45-47.

Shulman, L.S. (1986). Paradigms and research programs in the study of teaching: A contemporary perspective. In M.C. Wittrock (Ed.), Handbook of research on teaching (3 ${ }^{\text {rd }}$ ed., pp. 3-36). New York, NY: Macmillan Publishing Company.

Shulman, L.S. (1987). Knowledge and teaching: Foundations of the new reform. Harvard Educational Review, 57, 1-22.

Siedentop, D. (1972). On tilting at windmills while Rome burns. Quest, 18, 94-97.

Siedentop, D., \& Eldar, E. (1989). Expertise, experience, and effectiveness. Journal of Teaching in Physical Education, 8, 254-260.

Siedentop, D., \& Locke, L. (1997). Making a difference for physical education: What professors and practitioners must build together. Journal of Physical Education, Recreation and Dance, 68(4), 2533. 
Silberman, M. (1996). Active learning: 101 strategies to teach any subject. Boston, MA: Allyn and Bacon.

Stahl, N.N., \& Stahl, R.J. (1991). We can agree after all! Achieving consensus for a critical thinking component of a gifted program using the Delphi Technique [Electronic version]. Roeper Review, 14(2), 79-89.

Strand, B.N., Scantling, E., \& Johnson, M. (1997). Fitness education: Teaching concepts-based fitness in the schools. Scottsdale, AZ: Gorsuch Scarisbrick.

Suphawibul, M. (1991). Competencies for adapted physical educators in Thailand (Doctoral dissertation, Oregon State University, 1991). Dissertation Abstracts International, 53, 754.

Thomas, J.R., \& Thomas, K.T. (1989). What is motor development: Where does it belong? Quest, 41, 203-212.

Tinning, R.I. (1988). Student teaching and the pedagogy of necessity. Journal of Teaching in Physical Education, 7, 82-89.

Townsend, J.S., Wiegand, R.L., Bulger, S.M., Mohr, D.J., \& Metzler, M. (2002, April). Curricular issues in physical education teacher education. Paper presented at the Annual Convention of the American Alliance for Health, Physical Education, Recreation and Dance (AAHPERD), San Diego, CA.

Turoff, M., \& Hiltz, S.R. (1996). Computer-based Delphi processes. In M. Adler \& E. Ziglio (Eds.), Gazing into the oracle: The Delphi Method and its application to social policy and public health (pp. 56-85). Bristol, PA: Jessica Kingsley Publishers.

Twisk, J.W.R. (2001). Physical activity guidelines for children and adolescents: A critical review. Sports Medicine, 31, 617-627.

Twisk, J.W.R., Kemper, H.C.G., \& van Mechelen, W. (2002a). Prediction of cardiovascular disease risk factors later in life by physical activity and physical fitness in youth: Introduction. International Journal of Sports Medicine, 23, S5-S7.

Twisk, J.W.R., Kemper, H.C.G., \& van Mechelen, W. (2002b). Prediction of cardiovascular disease risk factors later in life by physical activity and physical fitness in youth: General comments and conclusions. International Journal of Sports Medicine, 23, S44-S49.

U.S. Department of Health and Human Services. (1996). Physical activity and health: A report of the Surgeon General. Atlanta, GA: U.S. Department of Health and Human Services, Center for Disease Control and Prevention, National Center for Chronic Disease Prevention and Health Promotion.

U.S. Department of Health and Human Services. (1997). Guidelines for school and community programs to promote lifelong physical activity among young people [Electronic version]. Morbidity and Mortality Weekly Report, 46(No. RR-6), 1-35.

U.S. Department of Health and Human Services. (2000). Promoting better health for young people through physical activity and sports. Atlanta, GA: U.S. Department of Health and Human Services and Department of Education. 
U.S. Department of Health and Human Services. (2001). The Surgeon General's call to action to prevent and decrease overweight and obesity. Washington, DC: U.S. Department of Health and Human Services, Public Health Service, and Office of the Surgeon General.

U.S. Department of Health and Human Services. (2002). Physical activity fundamental to preventing disease. Atlanta, GA: U.S. Department of Health and Human Services.

Van Donselaar, L., \& Leslie, D.K. (1990). Current and recommended preparation of physical education teachers in exercise physiology. Physical Educator, 47, 209-216.

Verner, M.E. (1991). Anatomy and physiology offerings in physical education professional preparation programs [Electronic version]. Physical Educator, 48, 80-86.

Vuori, I. (1995). Exercise and physical health: Musculoskeletal health and functional capabilities. Research Quarterly for Exercise and Sport, 66, 276-285.

Webber, L.S., Osganian, S.K., Feldman, H.A., Wu, M., McKenzie, T.L., Nichaman, M., Lytle, L.A., Edmundson, E., Cutler, J., Nader, P.R., \& Luepker, R.V. (1996). Cardiovascular risk factors among children after a 2 1/2-year intervention: The CATCH study. Preventive Medicine, 25, 432-441.

Welk, G.J., Wood, K., \& Morss, G. (2003). Parental influences on physical activity in children: An exploration of potential mechanisms. Pediatric Exercise Science, 15, 19-33.

Wiegand, R.L., Bulger, S.M., \& Mohr, D.J. (2004). Curricular issues in physical education teacher education. Manuscript submitted for publication.

Williams, A. (1998). Identification of content, priority, and methods of instructional delivery for a women's health component in an internal medicine residency program. Unpublished doctoral dissertation, West Virginia University, Morgantown.

Wilson, S., \& Williams, J.A. (2001). Student-centered case studies for teacher education. Journal of Physical Education, Recreation and Dance, 72(2), 49-53.

Woodfield, L., Duncan, M., Al-Nakeeb, Y., \& Jenkins, C. (2002). Sex, ethnic, and socio-economic differences in children's physical activity. Pediatric Exercise Science, 14, 277-285.

Ziglio, E. (1996). The Delphi Method and its contribution to decision-making. In M. Adler \& E. Ziglio (Eds.), Gazing into the oracle: The Delphi Method and its application to social policy and public health (pp. 3-33). Bristol, PA: Jessica Kingsley Publishers. 
Table 1 Sample Applications of the Delphi Method in Physical Education Research

\begin{tabular}{|c|c|c|}
\hline Citation & Category & Purpose \\
\hline Cicek \& Demirhan (2001) & Curriculum & $\begin{array}{l}\text { To validate a list of Turkish physical education } \\
\text { teacher education competencies. }\end{array}$ \\
\hline Hopkins (1999) & Prediction & $\begin{array}{l}\text { To forecast change in California State University } \\
\text { physical education and kinesiology departments. }\end{array}$ \\
\hline Schaeffer (1998) & Assessment & $\begin{array}{l}\text { To develop a testing instrument for assessing high } \\
\text { school coaches' knowledge of ergogenic aids. }\end{array}$ \\
\hline Good (1998) & Prediction & $\begin{array}{l}\text { To identify recommendations for change in the } \\
\text { physical education profession. }\end{array}$ \\
\hline Jacobs (1996) & Assessment & $\begin{array}{l}\text { To identify the critical elements to be employed in } \\
\text { assessing physical education teacher education. }\end{array}$ \\
\hline Cottone (1996) & Assessment & $\begin{array}{l}\text { To determine the beliefs and practices regarding } \\
\text { student assessment in athletic training education. }\end{array}$ \\
\hline Johnson (1992) & Prediction & $\begin{array}{l}\text { To forecast the financial problems that may occur in } \\
\text { Division II athletic and physical education programs. }\end{array}$ \\
\hline Suphawibul (1991) & Curriculum & $\begin{array}{l}\text { To determine a set of recommended competencies for } \\
\text { adapted physical educators in Thailand. }\end{array}$ \\
\hline Martin (1986) & Prediction & $\begin{array}{l}\text { To examine future curricular orientations of doctoral } \\
\text { programs in physical education. }\end{array}$ \\
\hline Cooper (1982) & Curriculum & $\begin{array}{l}\text { To develop an in-service manual and instructor's } \\
\text { guide for adapted physical educators. }\end{array}$ \\
\hline
\end{tabular}


Table 2 Exercise Science in PETE Delphi Panel Members

\begin{tabular}{|c|c|c|}
\hline Name & Institutional affiliation & Specialization \\
\hline Patty Freedson, Ph.D. & University of Massachusetts & Exercise Science \\
\hline Lawrence Golding, Ph.D. & University of Nevada - Las Vegas & Exercise Science \\
\hline Brian Goslin, Ph.D. & Oakland University & Exercise Science \\
\hline Terry Housh, Ph.D. & University of Nebraska - Lincoln & Exercise Science \\
\hline Ed Howley, Ph.D. & University of Tennessee & Exercise Science \\
\hline Kathy Hutcheson, Ed.D. & Colorado State University & Exercise Science \\
\hline Zebulon Kendrick, Ph.D. & Temple University & Exercise Science \\
\hline Lloyd Laubach, Ph.D. & University of Dayton & Exercise Science \\
\hline Barry McKeown, Ph.D. & University of Texas - Arlington & Exercise Science \\
\hline Gregory Welk, Ph.D. & Iowa State University & Exercise Science \\
\hline Donna Moore & Safford Middle School & Physical Education \\
\hline Danielle Parrish & Lake Ridge Academy & Physical Education \\
\hline Joan van Blom & Long Beach Unified School District & Physical Education \\
\hline Darrell Crase, Ph.D. & University of Memphis & Teacher Education \\
\hline Brett Everhart, Ed.D. & University of Texas - Arlington & Teacher Education \\
\hline Andrew Hawkins, Ph.D. & West Virginia University & Teacher Education \\
\hline Luke Kelly, Ph.D. & University of Virginia & Teacher Education \\
\hline Alan Lacy, Ed.D. & Illinois State University & Teacher Education \\
\hline Linda Rikard, Ed.D. & George Mason University & Teacher Education \\
\hline Brad Strand, Ph.D. & North Dakota State University & Teacher Education \\
\hline
\end{tabular}




\section{Table 3 Category I - Competencies Related to Anatomy and Biomechanics}

Exercise science competencies related to anatomy and biomechanics

Importance Relevance

$\begin{array}{llll}M & \% & \text { \% }\end{array}$

\begin{tabular}{|c|c|c|c|c|}
\hline 1. Knowledge of anatomy as it relates to exercise and health* & 4.60 & 95 & 4.40 & 95 \\
\hline 2. Knowledge of anatomy as it relates to team/individual sports & 4.00 & 85 & 3.95 & 80 \\
\hline 3. Knowledge of biomechanics as it relates to team/individual sports* & 4.45 & 100 & 4.35 & 95 \\
\hline 4. Knowledge of the basic structures of bone, skeletal muscle, and connective tissues & 4.45 & 95 & 3.95 & 80 \\
\hline 5. Knowledge of the basic anatomy of the cardiovascular system and respiratory system* & 4.70 & 100 & 4.35 & 100 \\
\hline $\begin{array}{l}\text { 6. Ability to identify the major bones and muscles: Major muscles include, but are not limited to: trapezius, } \\
\text { pectoralis major, latissimus dorsi, biceps, triceps, rectus abdominus, internal and external obliques, erector } \\
\text { spinae, gluteus maximus, quadriceps, hamstrings, adductors, abductors, and gastrocnemius }\end{array}$ & 4.60 & 95 & 3.85 & 70 \\
\hline $\begin{array}{l}\text { 7. Knowledge of the definition of the following terms: supination, pronation, flexion, extension, adduction, } \\
\text { abduction, hyperextension, rotation, circumduction, agonist, antagonist, and stabilizer }\end{array}$ & 4.35 & 90 & 3.55 & 55 \\
\hline 8. Ability to identify the joints of the body & 4.30 & 90 & 3.55 & 50 \\
\hline 9. Knowledge of the biomechanical aspects of exercise participation* & 4.47 & 90 & 4.11 & 80 \\
\hline 10. Knowledge to identify the plane in which each muscle action occurs & 3.37 & 45 & 2.58 & 10 \\
\hline $\begin{array}{l}\text { 11. Knowledge of the interrelationships among center of gravity, base of support, balance, stability, and proper } \\
\text { spinal alignment }\end{array}$ & 4.15 & 90 & 3.75 & 70 \\
\hline 12. Ability to describe the following curvatures of the spine: lordosis, scoliosis, and kyphosis & 3.65 & 55 & 3.30 & 45 \\
\hline $\begin{array}{l}\text { 13. Knowledge of and skill to demonstrate exercises designed to enhance muscular strength and/or endurance of } \\
\text { specific major muscle groups* }\end{array}$ & 4.89 & 95 & 4.79 & 90 \\
\hline 14. Knowledge of and skill to demonstrate exercise for enhancing musculoskeletal flexibility* & 4.75 & 100 & 4.65 & 95 \\
\hline 15. Knowledge to describe the myotatic stretch reflex & 3.42 & 50 & 2.74 & 20 \\
\hline 16. Knowledge to identify the primary action and joint range of motion for each major muscle group & 4.00 & 80 & 3.65 & 60 \\
\hline 17. Knowledge of functional anatomy and biomechanics* & 4.45 & 95 & 4.20 & 90 \\
\hline 18. Knowledg & 4.30 & 95 & 3.95 & 80 \\
\hline
\end{tabular}


Table 3 (continued)

\begin{tabular}{|c|c|c|c|c|}
\hline \multirow[b]{2}{*}{ Exercise science competencies related to anatomy and biomechanics } & \multicolumn{2}{|c|}{ Importance } & \multicolumn{2}{|c|}{ Relevance } \\
\hline & $M$ & $\%$ & $M$ & $\%$ \\
\hline 19. Ability to locate the anatomic landmarks for palpation of peripheral pulses & 3.89 & 70 & 3.63 & 60 \\
\hline $\begin{array}{l}\text { 20. Ability to locate the brachial artery and correctly place the cuff and stethoscope in position for blood pressure } \\
\text { measurement }\end{array}$ & 3.80 & 70 & 3.32 & 35 \\
\hline $\begin{array}{l}\text { 21. Ability to locate common sites for measurement of skinfold thickness and circumferences (for determination } \\
\text { of body composition and waist-hip ratio)* }\end{array}$ & 4.65 & 95 & 4.30 & 90 \\
\hline $\begin{array}{l}\text { 22. Knowledge of the biomechanical principles that underlie performance of the following activities: walking, } \\
\text { jogging, running, swimming, cycling, weight lifting, and carrying or moving objects* }\end{array}$ & 4.70 & 100 & 4.40 & 100 \\
\hline $\begin{array}{l}\text { 23. Knowledge of the influence of weight-bearing activity in childhood and adolescence on optimal bone growth } \\
\text { and development * }\end{array}$ & 4.60 & 100 & 4.20 & 95 \\
\hline
\end{tabular}

*Indicates a consensus of agreement was reached regarding both the importance and relevance of this competency. Each item had to meet the following criteria for consensus: (a) The item received a mean rating of at least four or higher in the areas of importance and relevance; and (b) the item received at least $75 \%$ of all individual ratings at the four level or higher. 


\section{Table 4 Category II - Competencies Related to Exercise Physiology}

Exercise science competencies related to exercise physiology

Importance Relevance

$M \quad \% \quad M \quad \%$

24. Basic knowledge of exercise physiology as it relates to exercise prescription*

$\begin{array}{llll}4.90 & 100 & 4.65 & 95\end{array}$

25. Ability to define aerobic and anaerobic metabolism*

$\begin{array}{llll}4.65 & 100 & 4.30 & 90\end{array}$

26. Knowledge of the role of aerobic and anaerobic energy systems in the performance of various activities*

$\begin{array}{llll}4.70 & 100 & 4.42 & 90\end{array}$

27. Knowledge of the following terms: ischemia, angina pectoris, tachycardia, bradycardia, arrhythmia,

$\begin{array}{llll}4.20 & 90 & 3.45 & 45\end{array}$
myocardial infarction, cardiac output, stroke volume, lactic acid, oxygen consumption, hyperventilation, systolic blood pressure, diastolic blood pressure, and anaerobic threshold

28. Knowledge of the role of carbohydrates, fats, and proteins as fuels for aerobic and anaerobic metabolism*

$\begin{array}{llll}4.35 & 95 & 4.05 & 85\end{array}$

29. Knowledge of the components of fitness: cardiorespiratory fitness, muscular strength, muscular endurance, flexibility, and body composition*

\begin{tabular}{|c|c|c|c|c|}
\hline $\begin{array}{l}\text { 30. Knowledge to describe normal cardiorespiratory responses to static and dynamic exercise in terms of heart } \\
\text { rate, blood pressure, and oxygen consumption* }\end{array}$ & 4.60 & 100 & 4.05 & 75 \\
\hline $\begin{array}{l}\text { 31. Knowledge of how heart rate, blood pressure, and oxygen consumption responses change with adaptation to } \\
\text { chronic exercise training* }\end{array}$ & 4.60 & 100 & 4.05 & 75 \\
\hline 32. Knowledge of the physiological adaptations associated with strength training* & 4.60 & 100 & 4.35 & 95 \\
\hline $\begin{array}{l}\text { 33. Ability to identify and apply to both groups and individuals methods used to monitor exercise intensity, } \\
\text { including heart rate and rating of perceived exertion* }\end{array}$ & 4.75 & 100 & 4.65 & 100 \\
\hline 34. Knowledge of the physiological principles related to warm-up and cool-down* & 4.75 & 100 & 4.74 & 95 \\
\hline 35. Knowledge of the common theories of muscle fatigue and delayed onset muscle soreness (DOMS) & 4.20 & 85 & 3.85 & 70 \\
\hline 36. Knowledge of exercise physiology including the role of aerobic & 4.65 & 95 & 4.45 & 100 \\
\hline
\end{tabular}
physiology, cardiovascular physiology, and respiratory physiology at rest and during exercise. In addition, demonstrate an understanding of the components of physical fitness, the effects of aerobic and strength and/or resistance training on the fitness components and the effects of chronic disease* 
Table 4 (continued)

Exercise science competencies related to exercise physiology

Importance Relevance

$M \quad \% \quad M \quad \%$

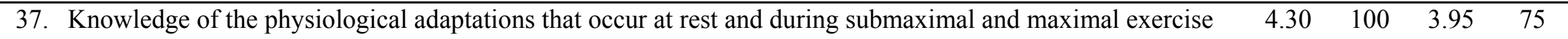
following chronic aerobic and anaerobic exercise training

\begin{tabular}{llllllll}
\hline 38. & Knowledge of the differences in cardiorespiratory response to acute graded exercise between conditioned and & 4.05 & 90 & 3.42 & 45
\end{tabular} unconditioned individuals

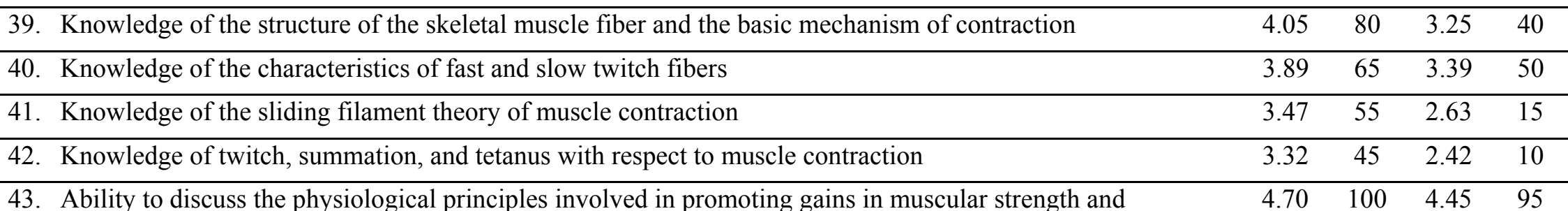
endurance*

44. Ability to define muscular fatigue as it relates to task, intensity, duration, and the accumulative effects of $\quad 4.25 \quad 90 \quad 4.10 \quad 75$ exercise*

45. Knowledge of the relationship between the number of repetitions, intensity, number of sets, and rest with $\quad 4.65 \quad 100 \quad 4.60 \quad 100$ regard to strength training*

$\begin{array}{llllll}\text { 46. Knowledge of the basic properties of cardiac muscle and normal pathways of conduction in heart } & 3.70 & 75 & 2.60 & 20\end{array}$

47. Knowledge of the response of these variables to acute exercise: heart rate, stroke volume, cardiac output, $\quad 3.90 \quad 80 \quad 3.45 \quad 50$ pulmonary ventilation, tidal volume, respiratory rate, arteriovenous oxygen difference

48. Knowledge of the differences in the cardiorespiratory responses to static exercise compared with dynamic $\quad 3.85 \quad 85 \quad 3.90 \quad 85$ exercise, including possible hazards and contraindications

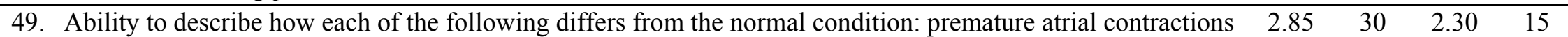
and premature ventricular contractions

51. Knowledge of and ability to describe the implications of ventilatory threshold (anaerobic threshold) as it $\quad \begin{array}{llll}4.00 & 80 & 3.42 & 45\end{array}$ relates to exercise training and cardiorespiratory assessment 
Table 4 (continued)

\begin{tabular}{|c|c|c|c|c|}
\hline \multirow[b]{2}{*}{ Exercise science competencies related to exercise physiology } & \multicolumn{2}{|c|}{ Importance } & \multicolumn{2}{|c|}{ Relevance } \\
\hline & $M$ & $\%$ & $M$ & $\%$ \\
\hline $\begin{array}{l}\text { 52. Knowledge of and ability to describe the physiological adaptations of the respiratory system that occur at rest } \\
\text { and during submaximal/maximal exercise following chronic aerobic/anaerobic training }\end{array}$ & 4.10 & 90 & 3.55 & 55 \\
\hline $\begin{array}{l}\text { 53. Ability to describe how each of the following differs from the normal condition: dyspnea, hypoxia, and } \\
\text { hypoventilation }\end{array}$ & 3.10 & 45 & 2.42 & 15 \\
\hline $\begin{array}{l}\text { 54. Knowledge of and ability to discuss the physiological basis of the major components of physical fitness: } \\
\text { flexibility, cardiovascular fitness, muscular strength/endurance, and body composition* }\end{array}$ & 4.90 & 100 & 4.80 & 100 \\
\hline 55. Ability to explain how the principle of specificity relates to the components of fitness* & 4.80 & 100 & 4.60 & 100 \\
\hline 56. Ability to explain the concept of detraining or reversibility and its implications in fitness programs* & 4.20 & 90 & 4.10 & 85 \\
\hline $\begin{array}{l}\text { 57. Ability to discuss the physical and psychological signs of overtraining and provide recommendations for } \\
\text { these problems }\end{array}$ & 4.15 & 90 & 3.75 & 75 \\
\hline $\begin{array}{l}\text { 58. Ability to describe the physiological and metabolic responses to exercise associated with chronic disease } \\
\text { (heart disease, hypertension, diabetes mellitus, and pulmonary disease) }\end{array}$ & 3.75 & 75 & 3.45 & 50 \\
\hline
\end{tabular}

*Indicates a consensus of agreement was reached regarding both the importance and relevance of this competency. Each item had to meet the following criteria for consensus: (a) The item received a mean rating of at least four or higher in the areas of importance and relevance; and (b) the item received at least $75 \%$ of all individual ratings at the four level or higher. 


\section{Table 5 Category III - Competencies Related to Human Development and Aging}

Exercise science competencies related to human development and aging

Importance Relevance

$M \quad \% \quad M \quad \%$

\begin{tabular}{|c|c|c|c|c|}
\hline $\begin{array}{l}\text { 59. Knowledge of the benefits and risks associated with exercise training in prepubescent and postpubescent } \\
\text { youth* }\end{array}$ & 4.74 & 95 & 4.68 & 95 \\
\hline 60. Knowledge of the benefits and precautions associated with resistance and endurance training in older adults & 3.63 & 70 & 2.53 & 15 \\
\hline 61. Ability to describe specific leadership techniques appropriate for working with participants of all ages & 4.06 & 70 & 3.78 & 70 \\
\hline 62. Knowledge of the changes that occur during growth and development from childhood to old age* & 4.58 & 90 & 4.37 & 90 \\
\hline 63. Ability to modify cardiovascular and resistance exercises based on age and physical condition* & 4.42 & 90 & 4.47 & 90 \\
\hline $\begin{array}{l}\text { 64. Knowledge of and ability to describe the changes that occur in maturation from childhood to adulthood for } \\
\text { the following: skeletal muscle, bone structure, reaction time, coordination, heat and cold tolerance, maximal } \\
\text { oxygen consumption, strength, flexibility, body composition, resting and maximal heart rate, and resting and } \\
\text { maximal blood pressure }\end{array}$ & 4.47 & 95 & 3.95 & 70 \\
\hline $\begin{array}{l}\text { 65. Knowledge of the effect of the aging process on the musculoskeletal and cardiovascular structure and } \\
\text { function at rest, during exercise, and during recovery }\end{array}$ & 3.47 & 60 & 2.89 & 25 \\
\hline $\begin{array}{l}\text { 66. Ability to characterize the differences in the development of an exercise prescription for children, } \\
\text { adolescents, and older participants* }\end{array}$ & 4.53 & 90 & 4.16 & 75 \\
\hline $\begin{array}{l}\text { 67. Knowledge of and ability to describe the unique adaptations to exercise training in children, adolescents, and } \\
\text { older participants with regard to strength, functional capacity, and motor skills* }\end{array}$ & 4.47 & 85 & 4.32 & 90 \\
\hline $\begin{array}{l}\text { 68. Knowledge of the common orthopedic and cardiovascular considerations for older participants and the ability } \\
\text { to describe modifications in exercise prescription that are indicated }\end{array}$ & 2.84 & 20 & 2.33 & 10 \\
\hline 69. Knowledge of the health-related benefits of regular physical activity for people of all ages* & 4.79 & 95 & 4.84 & 95 \\
\hline
\end{tabular}

*Indicates a consensus of agreement was reached regarding both the importance and relevance of this competency. Each item had to meet the following criteria for consensus: (a) The item received a mean rating of at least four or higher in the areas of importance and relevance; and (b) the item received at least $75 \%$ of all individual ratings at the four level or higher. 
Table 6 Category IV - Competencies Related to Pathophysiology and Risk Factors

Exercise science competencies related to pathophysiology and risk factors

Importance Relevance

Exercise science conpetencies related to pathophysiology and risk factors

70. Knowledge of cardiovascular, respiratory, metabolic, and musculoskeletal risk factors that may require further evaluation by medical or allied health professionals before participation in physical activity

71. Ability to determine those risk factors that may be favorably modified by physical activity habits*

72. Knowledge to define the following terms: total cholesterol (TC), high-density lipoprotein cholesterol (HDLC), TC/HDL-C ratio, low-density lipoprotein cholesterol (LDL-C), triglycerides, hypertension, and artherosclerosis

73. Knowledge of plasma cholesterol levels for adults as recommended by the National Cholesterol Educatio Program (NCEP II)

74. Knowledge of the pathophysiology of atherosclerosis and how this process is influenced by physical activity

75. Knowledge of the risk factor concept of coronary artery disease (CAD) and the influence of heredity and lifestyle on the development of CAD*

76. Knowledge of the artherosclerotic process, the factors involved in its genesis and progression, and the potential role of exercise training in treatment

\footnotetext{
77. Ability to discuss in detail how lifestyle factors, including nutrition, physical activity, and heredity, influence $\quad 4.20 \quad 95 \quad 4.00 \quad 75$ lipid and lipoprotein profiles*

78. Knowledge of cardiovascular risk factors or conditions that may require consultation with medical personnel $\quad 4.2 \quad 90 \quad 3.75 \quad 60$ before testing or training, including inappropriate changes in resting or exercise heart rate and blood pressure, new onset discomfort in chest, neck, shoulder, or arm, changes in the pattern of discomfort during rest or exercise, fainting or dizzy spells, and claudication

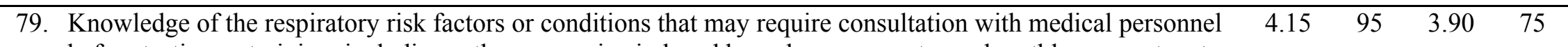
before testing or training, including asthma, exercise-induced bronchospasm, extreme breathlessness at rest or during exercise, bronchitis, and emphysema

80. Knowledge of the metabolic risk factors or conditions that may require consultation with medical personnel before testing or training, including bodyweight more than $20 \%$ above optimal, BMI $>30$, thyroid disease, diabetes or glucose intolerance, and hypoglycemia

81. Knowledge of the musculoskeletal risk factors or conditions that may require consultation with medical personnel before testing or training, including acute or chronic back pain, osteoarthritis, rheumatoid arthritis, osteoporosis, tendonitis, and low back pain

$\begin{array}{llll}3.95 & 85 & 3.84 & 60\end{array}$

$\begin{array}{llll}3.90 & 80 & 3.20 & 30\end{array}$
}




\section{Table 6 (continued)}

\begin{tabular}{|c|c|c|c|c|}
\hline \multirow[b]{2}{*}{ Exercise science competencies related to pathophysiology and risk factors } & \multicolumn{2}{|c|}{ Importance } & \multicolumn{2}{|c|}{ Relevance } \\
\hline & $M$ & $\%$ & $M$ & $\%$ \\
\hline $\begin{array}{l}\text { 82. Knowledge of persons with physical and mental disabilities and the potential influence on risk for chronic } \\
\text { degenerative disease }\end{array}$ & 3.79 & 70 & 3.26 & 40 \\
\hline $\begin{array}{l}\text { 83. Knowledge of the factors that increase risk for osteoporosis and the preventive measures that can be followed } \\
\text { to decrease risk }\end{array}$ & 3.85 & 80 & 3.11 & 75 \\
\hline
\end{tabular}

*Indicates a consensus of agreement was reached regarding both the importance and relevance of this competency. Each item had to meet the following criteria for consensus: (a) The item received a mean rating of at least four or higher in the areas of importance and relevance; and (b) the item received at least $75 \%$ of all individual ratings at the four level or higher. 


\section{Table 7 Category V - Competencies Related to Human Behavior and Psychology}

Exercise science competencies related to human behavior and psychology

Importance Relevance

$M \quad \% \quad M \quad \%$

84. Ability to identify and define at least five behavioral strategies to enhance exercise and health behavior change (i.e., reinforcement, goal setting, social support)*

85. Ability to list and define the five important elements that should be included in a health counseling session

86. Knowledge of specific techniques to enhance motivation (e.g., posters, recognition, bulletin boards, games, competitions). Define extrinsic and intrinsic reinforcement and give examples of each*

\begin{tabular}{|c|c|c|c|c|}
\hline 87. Knowledge of the stages of motivational readiness & 3.72 & 75 & 3.59 & 60 \\
\hline $\begin{array}{l}\text { 88. Ability to list and describe three counseling approaches that may assist less motivated clients to increase their } \\
\text { physical activity }\end{array}$ & 3.50 & 55 & 3.24 & 40 \\
\hline $\begin{array}{l}\text { 89. Ability to list and describe the specific strategies aimed at encouraging initiation, adherence, and return to } \\
\text { participation in an exercise program }\end{array}$ & 4.11 & 75 & 3.76 & 55 \\
\hline 90. Knowledge of symptoms of anxiety and depression that may necessitate referral & 3.47 & 45 & 2.94 & 30 \\
\hline $\begin{array}{l}\text { 91. Knowledge of the potential symptoms and causal factors of test anxiety (i.e., performance, appraisal threat } \\
\text { during exercise testing) and how it may affect physiological responses to testing }\end{array}$ & 3.33 & 45 & 2.76 & 25 \\
\hline $\begin{array}{l}\text { 92. Ability to facilitate the development of positive attitudes and perceptions regarding physical activity and } \\
\text { exercise* }\end{array}$ & 4.39 & 80 & 4.47 & 75 \\
\hline $\begin{array}{l}\text { 93. Knowledge of the use of goal setting and behavioral contracts as key components of the behavior change } \\
\text { process* }\end{array}$ & 4.20 & 95 & 4.10 & 85 \\
\hline $\begin{array}{l}\text { 94. Knowledge of the psycho-social determinants of physical activity (e.g., parental/peer influence, self-efficacy, } \\
\text { self-perception) }\end{array}$ & 4.15 & 95 & 3.80 & 75 \\
\hline $\begin{array}{l}\text { 95. Knowledge of the environmental determinants of physical activity (e.g., television/computers, access to } \\
\text { exercise facilities, neighborhood safety)* }\end{array}$ & 4.30 & 90 & 4.05 & 75 \\
\hline 5 & 44 & 95 & 4.45 & \\
\hline
\end{tabular}

exercise environment* 


\section{Table 7 (continued)}

\begin{tabular}{|c|c|c|c|c|}
\hline \multirow[b]{2}{*}{ Exercise science competencies related to human behavior and psychology } & \multicolumn{2}{|c|}{ Importance } & \multicolumn{2}{|c|}{ Relevance } \\
\hline & $M$ & $\%$ & $M$ & $\%$ \\
\hline $\begin{array}{l}\text { 97. Knowledge of various educational theories and instructional methods that can be used in the area of physical } \\
\text { activity promotion* }\end{array}$ & 4.15 & 80 & 4.30 & 90 \\
\hline 98. Knowledge of the psychological benefits associated with participation in regular physical activity* & 4.35 & 85 & 4.1 & 80 \\
\hline $\begin{array}{l}\text { 99. Knowledge of the psychological variables associated with sport performance (e.g., anxiety, motivation, } \\
\text { burnout) }\end{array}$ & 3.75 & 75 & 3.45 & 55 \\
\hline 100. Knowledge of the signs/symptoms of disordered eating and the appropriate procedures for referral* & 4.45 & 100 & 4.20 & 85 \\
\hline
\end{tabular}

*Indicates a consensus of agreement was reached regarding both the importance and relevance of this competency. Each item had to meet the following criteria for consensus: (a) The item received a mean rating of at least four or higher in the areas of importance and relevance; and (b) the item received at least $75 \%$ of all individual ratings at the four level or higher. 


\section{Table 8 Category VI - Competencies Related to Health Appraisal and Fitness Testing}

Exercise science competencies related to health appraisal and fitness testing

Importance Relevance

$M \quad \% \quad M \quad \%$

101. Knowledge of the importance of a health/medical history

102. Knowledge of the value of a medical clearance prior to exercise participation

103. Skill to measure pulse rate accurately both at rest and during exercise*

104. Knowledge, skills, and abilities to assess the health status of individuals and the ability to conduct fitness testing*

105. Ability to obtain a health history and risk appraisal that includes past and current medical history, family history of cardiac disease, orthopedic limitations, prescribed medications, activity patterns, nutritional habits, stress and anxiety levels, and smoking and alcohol use

106. Ability to describe the categories of participants who should receive medical clearance prior to administration $\quad 3.65 \quad 55 \quad 3.10 \quad 35$ of an exercise test or participation in an exercise program

\begin{tabular}{|c|c|c|c|c|}
\hline 107. Ability to identify relative and absolute contraindications to exercise testing or participation & 3.75 & 65 & 3.55 & 60 \\
\hline 108. Ability to discuss the limitations of informed consent and medical clearance prior to exercise testing & 3.65 & 60 & 2.75 & 20 \\
\hline 109. Ability to obtain informed consent & 3.84 & 65 & 3.26 & 40 \\
\hline $\begin{array}{l}\text { 110. Ability to explain the purpose and procedures for monitoring clients prior to, during, } \\
\text { cardiorespiratory fitness testing }\end{array}$ & 3.85 & 75 & 3.30 & 45 \\
\hline
\end{tabular}

111. Skill in instructing participants in the use of equipment and test procedures*

$\begin{array}{llll}4.40 & 95 & 4.40 & 95\end{array}$

112. Ability to describe the purpose of testing, select an appropriate submaximal or maximal protocol, and conduct $\quad 3.55 \quad 55 \quad 2.70 \quad 10$ an assessment of cardiovascular fitness on the cycle ergometer or the treadmill

113. Skill in accurately measuring heart rate, blood pressure, and obtaining rating of perceived exertion (RPE) at $\quad \begin{array}{llll}4.20 & 90 & 3.70 & 55\end{array}$ rest and during exercise according to established guidelines

\begin{tabular}{|c|c|c|c|c|}
\hline $\begin{array}{l}\text { 114. Ability to locate and measure skinfold sites, skeletal diameters, and girth measurements used for estimating } \\
\text { body composition* }\end{array}$ & 4.50 & 95 & 4.05 & 80 \\
\hline $\begin{array}{l}\text { 115. Ability to describe the purpose of testing, select appropriate protocols, and conduct assessments of muscl } \\
\text { strength, muscular endurance, and flexibility* }\end{array}$ & 4.55 & 95 & 4.40 & 90 \\
\hline 116. Skill in various techniques of assessing body composition & 4.20 & 85 & 4.00 & 70 \\
\hline
\end{tabular}


Exercise science competencies related to health appraisal and fitness testing

Importance Relevance

$M \quad \% \quad M \quad \%$

117. Knowledge of the advantages/disadvantages and limitations of the various body composition techniques

118. Ability to interpret information obtained from cardiorespiratory fitness tests and muscular strength/endurance, flexibility, and body composition tests for apparently healthy individuals and those with stable disease

\begin{tabular}{|c|c|c|c|c|}
\hline $\begin{array}{l}\text { 119. Ability to identify appropriate criteria for terminating a fitness evaluation and demonstrate proper procedures } \\
\text { to be followed after discontinuing such a test }\end{array}$ & 3.85 & 70 & 3.45 & 45 \\
\hline $\begin{array}{l}\text { 120. Ability to modify protocols and procedures for cardiorespiratory fitness tests in children, adolescents, and } \\
\text { older adults* }\end{array}$ & 4.40 & 90 & 4.00 & 75 \\
\hline $\begin{array}{l}\text { 121. Ability to conduct fitness appraisals for populations with physical and mental disabilities (e.g., mental } \\
\text { retardation) which may require alternative testing techniques }\end{array}$ & 3.85 & 75 & 3.63 & 55 \\
\hline $\begin{array}{l}\text { 122. Knowledge of the steps needed to prepare children for physical fitness testing (e.g., teaching test purpose, } \\
\text { protocol, pacing)* }\end{array}$ & 4.55 & 95 & 4.60 & 90 \\
\hline 123. Knowledge of the differences between norm and criterion referenced fitness tests* & 4.40 & 85 & 4.30 & 80 \\
\hline $\begin{array}{l}\text { 124. Knowledge of the role of physical fitness testing in achieving national and state standards for physical } \\
\text { education* }\end{array}$ & 4.45 & 95 & 4.37 & 85 \\
\hline 125. Ability to use heart rate monitors for monitoring intensity during exercise* & 4.10 & 80 & 4.05 & 75 \\
\hline 126. Knowledge of the NIH definitions of obesity and overweight using body mass index (BMI)* & 4.20 & 90 & 4.05 & 75 \\
\hline 127. Ability to use pedometers for monitoring physical activity participation* & 4.35 & 85 & 4.37 & 85 \\
\hline 128. Knowledge of bronchodilators and their principal effects on exercise testing and prescription & 3.15 & 35 & 3.00 & 30 \\
\hline 129. Knowledge of hypoglycemics and their principal effects on exercise testing and prescription & 3.05 & 35 & 2.74 & 20 \\
\hline 130. Knowledge of psychotropics and their principal effects on exercise testing and prescription & 2.85 & 20 & 2.42 & 0 \\
\hline 131. Knowledge of vasodilators and their principal effects on exercise testing and prescription & 2.90 & 20 & 2.53 & 10 \\
\hline $\begin{array}{l}\text { 132. Ability to identify the effects of the following substances on exercise response: antihistamines, tranquilizers, } \\
\text { alcohol, diet pills, cold tablets, caffeine, and nicotine }\end{array}$ & 3.30 & 45 & 3.05 & 35 \\
\hline
\end{tabular}

*Indicates a consensus of agreement was reached regarding both the importance and relevance of this competency. Each item had to meet the following criteria for consensus: (a) The item received a mean rating of at least four or higher in the areas of importance and relevance; and (b) the item received at least $75 \%$ of all individual ratings at the four level or higher. 


\section{Table 9 Category VII - Safety, Injury Prevention, and Emergency Care}

Exercise science competencies related to safety, injury prevention, and emergency care

Importance Relevance

$M \quad \% \quad M \quad \%$

133. Knowledge of and skill in obtaining basic life support and cardiopulmonary certification*

$\begin{array}{llll}5.00 & 100 & 4.85 & 95 \\ 4.95 & 100 & 4.85 & 95\end{array}$

134. Knowledge of appropriate emergency procedures (i.e., telephone procedures, written emergency procedures, personnel responsibilities) in the group exercise setting*

135. Knowledge of basic first aid procedures for exercise-related injuries, such as bleeding, strains/sprains, fractures, and exercise intolerance (dizziness, syncope, heat injury)*

\begin{tabular}{|c|c|c|c|c|}
\hline 136. Knowledge of basic precautions taken in a group exercise setting to ensure participant safety* & 4.70 & 100 & 4.55 & 95 \\
\hline 137. Ability to identify the physical and physiological signs and symptoms of overtraining & 4.05 & 85 & 3.60 & 60 \\
\hline $\begin{array}{l}\text { 138. Ability to list the effects of temperature, humidity, altitude, and pollution on physiological response to } \\
\text { exercise* }\end{array}$ & 4.60 & 100 & 4.20 & 85 \\
\hline $\begin{array}{l}\text { 139. Knowledge of the following terms: shin splints, sprain, strain, tennis elbow, bursitis, stress fracture, tendonitis, } \\
\text { patellar femoral pain syndrome, low back pain, plantar fasciitis, and rotator cuff tendonitis }\end{array}$ & 4.35 & 90 & 3.75 & 80 \\
\hline 140. Skill to demonstrate exercises used for people with low back pain & 4.05 & 85 & 3.40 & 60 \\
\hline 142. Skill in demonstrating appropriate emergency procedures during exercise testing and/or training & 4.16 & 80 & 3.68 & 60 \\
\hline $\begin{array}{l}\text { 143. Knowledge of safety plans, emergency procedures, and first aid techniques needed during fitness evaluations, } \\
\text { exercise testing, and exercise training* }\end{array}$ & 4.30 & 85 & 4.15 & 80 \\
\hline
\end{tabular}
cardiovascular/pulmonary complications (e.g., tachycardia, bradycardia, hypotension/hypertension, tachypnea), and metabolic abnormalities (e.g., fainting/syncope, hypoglycemia/hyperglycemia, hypothermia/hyperthermia) 
Table 9 (continued)

\begin{tabular}{|c|c|c|c|c|}
\hline \multirow[b]{2}{*}{ Exercise science competencies related to safety, injury prevention, and emergency care } & \multicolumn{2}{|c|}{ Importance } & \multicolumn{2}{|c|}{ Relevance } \\
\hline & $M$ & $\%$ & $M$ & $\%$ \\
\hline $\begin{array}{l}\text { 147. Knowledge of the initial management and first aid techniques associated with open wounds, musculoskeletal } \\
\text { injuries, cardiovascular/pulmonary complications, and metabolic disorders* }\end{array}$ & 4.55 & 95 & 4.35 & 95 \\
\hline $\begin{array}{l}\text { 148. Knowledge of the components of an equipment maintenance/repair program and how it may be used to } \\
\text { evaluate the condition of exercise equipment to reduce potential risk of injury }\end{array}$ & 3.60 & 65 & 3.30 & 45 \\
\hline
\end{tabular}

*Indicates a consensus of agreement was reached regarding both the importance and relevance of this competency. Each item had to meet the following criteria for consensus: (a) The item received a mean rating of at least four or higher in the areas of importance and relevance; and (b) the item received at least $75 \%$ of all individual ratings at the four level or higher. 


\section{Table 10 Category VIII - Exercise Programming}

Exercise science competencies related to exercise programming

Importance Relevance

$M \quad \% \quad M \quad \%$

149. Knowledge of the recommended intensity, duration, frequency, and type of physical activity necessary for development of cardiorespiratory fitness in an apparently healthy population*

150. Ability to differentiate between the amount of physical activity required for health benefits and the amount of $\quad 4.75 \quad 100 \quad 4.55 \quad 90$ exercise required for fitness development*

151. Ability to describe exercises designed to enhance muscular strength and/or endurance of specific major muscle $\quad 4.80 \quad 100 \quad 4.75 \quad \begin{array}{lll}100\end{array}$ groups*

152. Knowledge of the principles of overload, specificity, and progression and how they relate to exercise $\quad \begin{array}{llll}4.80 & 100 & 4.60 & 95\end{array}$ programming*

153. Skill to teach and demonstrate appropriate exercises used in the warm-up and cool-down of a variety of group $\quad 4.60 \quad 95 \quad 4.60 \quad 95$ exercise classes*

154. Ability to teach the components of an exercise session (i.e., warm-up, aerobic stimulus phase, cool-down, $\quad 4.70 \quad 100 \quad 4.65 \quad 100$ muscular strength/endurance, flexibility)*

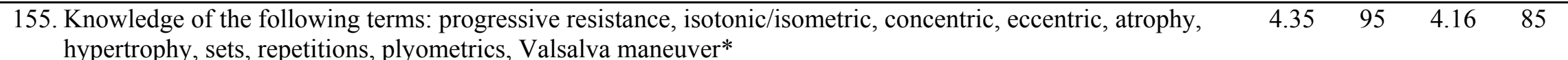

156. Skill to teach class participants how to monitor intensity of exercise using heart rate and rating of perceived $\quad 4.45 \quad 95 \quad 4.32 \quad 85$ exertion (RPE)*

\begin{tabular}{|c|c|c|c|c|}
\hline 157. Skill to teach participants how to use RPE and heart rate to adjust the intensity of the exercise session* & 4.35 & 95 & 4.33 & 80 \\
\hline $\begin{array}{l}\text { 158. Ability to calculate training heart rates using two methods: percent of age-predicted maximum heart rate and } \\
\text { heart rate reserve (Karvonen)* }\end{array}$ & 4.35 & 95 & 4.16 & 85 \\
\hline $\begin{array}{l}\text { 159. Skill to teach and demonstrate appropriate modifications in specific exercises for the following groups: older } \\
\text { adults, pregnant and postnatal women, obese persons, and persons with low back pain }\end{array}$ & 3.40 & 60 & 2.60 & 20 \\
\hline
\end{tabular}
weights, bands, resistance bars, and water exercise equipment* 


\begin{tabular}{|c|c|c|c|c|}
\hline $\begin{array}{l}\text { 161. Ability to recognize proper and improper technique in the use of cardiovascular conditioning equipment (e.g., } \\
\text { steps, cycles, slides)* }\end{array}$ & 4.45 & 90 & 4.40 & 90 \\
\hline 162. Skill to teach and demonstrate appropriate exercises for improving range of motion of major joints* & 4.50 & 95 & 4.47 & 95 \\
\hline $\begin{array}{l}\text { 164. Ability to teach a progression of exercises for all major muscle groups to improve muscular strength and } \\
\text { endurance* }\end{array}$ & 4.65 & 100 & 4.60 & 100 \\
\hline 165. Knowledge to describe the various types of interval, continuous, and circuit training programs* & 4.55 & 100 & 4.50 & 100 \\
\hline 167. Ability to communicate effectively with exercise participants in the group exercise session* & 4.60 & 100 & 4.65 & 100 \\
\hline 168. Knowledge to describe partner resistance exercises that can be used in a group class setting* & 4.40 & 90 & 4.20 & 90 \\
\hline 169. Ability to demonstrate techniques for accommodating various fitness levels within the same class* & 4.40 & 90 & 4.55 & 90 \\
\hline $\begin{array}{l}\text { 173. Skill to demonstrate the proper form, alignment, and technique in typical exercises used in warm-up, stimulus, } \\
\text { muscle conditioning, and cool-down phases of the group session* }\end{array}$ & 4.30 & 90 & 4.30 & 90 \\
\hline 174. Ability to evaluate specific exercises in terms of safety and effectiveness for various participants* & 4.40 & 85 & 4.55 & 90 \\
\hline $\begin{array}{l}\text { 175. Ability to demonstrate a familiarity with a variety of group exercise formats (e.g., traditional, step, slide, } \\
\text { muscle conditioning, flexibility, indoor cycling, water fitness, walking)* }\end{array}$ & 4.15 & 90 & 4.10 & 85 \\
\hline $\begin{array}{l}\text { 176. Knowledge, skills, and abilities to prescribe and administer exercise programs for apparently healthy } \\
\text { individuals,individuals at higher risk and individuals with known disease }\end{array}$ & 3.60 & 70 & 3.30 & 45 \\
\hline
\end{tabular}


Exercise science competencies related to exercise programming

Importance Relevance

$M \quad \% \quad M \quad \%$

177. Ability to design, implement, and evaluate individualized and group exercise programs based on health history $\quad 4.00 \quad 80 \quad 3.30 \quad 45$ and physical fitness assessments

\begin{tabular}{|c|c|c|c|c|}
\hline 178. Ability to modify exercises based on age and physical condition* & 4.40 & 100 & 4.15 & 85 \\
\hline $\begin{array}{l}\text { 179. Knowledge, skills, and abilities to calculate energy cost, VO2, METs, and target heart rates and apply the } \\
\text { information to an exercise prescription }\end{array}$ & 4.05 & 90 & 3.20 & 45 \\
\hline $\begin{array}{l}\text { 180. Ability to convert weights from pounds }(\mathrm{lb}) \text { to kilograms }(\mathrm{kg}) \text { and speed from miles per hour }(\mathrm{MPH}) \text { to meters } \\
\text { per minute }(\mathrm{m} \cdot \mathrm{min}-1)\end{array}$ & 3.50 & 60 & 3.00 & 35 \\
\hline 181. Ability to convert METs to VO2 expressed as $\mathrm{mL} \cdot \mathrm{kg}-1 \cdot \mathrm{min}-1, \mathrm{~L} \cdot \mathrm{min}-1$, and/or $\mathrm{mL} \cdot \mathrm{kg} \mathrm{FFW}-1 \cdot \mathrm{min}-1$ & 3.15 & 40 & 2.10 & 10 \\
\hline $\begin{array}{l}\text { 182. Ability to calculate the energy cost in METs and kilocalories for given exercise intensities in stepping exercise, } \\
\text { cycle ergometry, and during horizontal and graded walking and running }\end{array}$ & 3.45 & 60 & 2.60 & 20 \\
\hline $\begin{array}{l}\text { 184. Ability to prescribe exercise intensity based on } \mathrm{VO} 2 \text { data for different modes of exercise, including graded and } \\
\text { horizontal running and walking, cycling, and stepping exercise }\end{array}$ & 3.60 & 65 & 2.60 & 15 \\
\hline $\begin{array}{l}\text { 185. Ability to explain and implement exercise prescription guidelines for apparently health clients, increased risk } \\
\text { clients, and clients with controlled disease }\end{array}$ & 3.40 & 65 & 2.95 & 35 \\
\hline $\begin{array}{l}\text { 186. Ability to adapt frequency, intensity, duration, mode, progression, level of supervision, and monitoring } \\
\text { techniques in exercise programs for patients with controlled chronic disease (heart disease, diabetes mellitus, } \\
\text { obesity, hypertension), musculoskeletal problems, pregnancy and/or postpartum and exercise-induced asthma }\end{array}$ & 2.95 & 30 & 2.60 & 15 \\
\hline $\begin{array}{l}\text { 188. Skill in the use of various methods for establishing and monitoring levels of exercise intensity, including heart } \\
\text { rate, RPE, and METs }\end{array}$ & 4.15 & 85 & 3.75 & 70 \\
\hline $\begin{array}{l}\text { 189. Knowledge of special precautions and modifications of exercise programming for participation at altitude, } \\
\text { different ambient temperatures, humidity, and environmental pollution }\end{array}$ & 4.25 & 100 & 3.80 & 80 \\
\hline
\end{tabular}




\begin{tabular}{|c|c|c|c|c|}
\hline 191. Ability to evaluate flexibility and prescribe appropriate flexibility exercises for all major muscle groups* & 4.65 & 100 & 4.55 & 95 \\
\hline $\begin{array}{l}\text { 192. Knowledge of the importance of recording exercise sessions and performing periodic evaluations to assess } \\
\text { changes in fitness status* }\end{array}$ & 4.20 & 95 & 4.25 & 90 \\
\hline $\begin{array}{l}\text { 193. Knowledge of the advantages and disadvantages of implementation of interval, continuous, and circuit training } \\
\text { programs }\end{array}$ & 4.37 & 90 & 3.89 & 70 \\
\hline 194. Ability to design training programs using interval, continuous, and circuit training programs* & 4.26 & 80 & 4.16 & 80 \\
\hline $\begin{array}{l}\text { 195. Ability to discuss the advantages and disadvantages of various commercial exercise equipment in developing } \\
\text { cardiorespiratory fitness, muscular strength, and muscular endurance }\end{array}$ & 3.84 & 75 & 3.42 & 50 \\
\hline $\begin{array}{l}\text { 196. Knowledge of the types of exercise programs available in the community and how these programs are } \\
\text { appropriate for various populations }\end{array}$ & 4.00 & 75 & 3.68 & 60 \\
\hline 197. Ability to include participants with different individual needs into a group exercise environment* & 4.21 & 90 & 4.32 & 90 \\
\hline 198. Knowledge of the concept of periodization as it relates to strength and endurance exercise programming & 3.94 & 80 & 3.39 & 55 \\
\hline 199. Knowledge of the special concerns and guidelines recommended for children and adolescent strength training* & 4.89 & 95 & 4.63 & 95 \\
\hline
\end{tabular}

*Indicates a consensus of agreement was reached regarding both the importance and relevance of this competency. Each item had to meet the following criteria for consensus: (a) The item received a mean rating of at least four or higher in the areas of importance and relevance; and (b) the item received at least $75 \%$ of all individual ratings at the four level or higher. 


\section{Table 11 Category IX - Nutrition and Weight Management}

Exercise science competencies related to nutrition and weight management

Importance Relevance

$M \quad \% \quad M \quad \%$

201. Knowledge to define the following terms: obesity, overweight, percent fat, lean body mass, anorexia nervosa, bulimia, and body fat distribution*

\begin{tabular}{|c|c|c|c|c|}
\hline 202. Knowledge of the relationship between body composition and health* & 4.68 & 95 & 4.42 & 85 \\
\hline $\begin{array}{l}\text { 203. Knowledge of the effects of diet plus exercise, diet alone, and exercise alone as methods for modifying body } \\
\text { composition* }\end{array}$ & 4.74 & 95 & 4.58 & 90 \\
\hline 204. Knowledge of the importance of an adequate daily energy intake for healthy weight management* & 4.47 & 90 & 4.53 & 90 \\
\hline 205. Ability to differentiate between fat soluble and water-soluble vitamins & 3.37 & 50 & 2.63 & 15 \\
\hline 206. Ability to describe the importance of maintaining normal hydration before, during, and after exercise* & 4.79 & 95 & 4.84 & 95 \\
\hline 207. Knowledge of the USDA Food Pyramid* & 4.37 & 90 & 4.21 & 90 \\
\hline 208. Knowledge of the importance of calcium and iron in women's health & 4.00 & 85 & 3.84 & 75 \\
\hline $\begin{array}{l}\text { 209. Ability to describe the myths and consequences associated with inappropriate weight loss methods (e.g., } \\
\text { saunas, vibrating belts, body wraps, electric simulators, sweat suits, fad diets)* }\end{array}$ & 4.68 & 95 & 4.47 & 95 \\
\hline 210. Knowledge of the number of kilocalories in one gram of carbohydrate, fat, protein, and alcohol & 3.95 & 75 & 3.21 & 40 \\
\hline 211. Knowledge of the number of kilocalories equivalent to losing 1 pound of body fat & 3.95 & 70 & 3.53 & 55 \\
\hline $\begin{array}{l}\text { 212. Knowledge, skills, and abilities to provide information concerning nutrition and the role of diet and exercise on } \\
\text { body composition and weight control* }\end{array}$ & 4.63 & 95 & 4.47 & 95 \\
\hline $\begin{array}{l}\text { 213. Ability to describe the health implications of variation in body fat distribution patterns and the significance of } \\
\text { waist to hip ratio }\end{array}$ & 4.05 & 80 & 3.74 & 60 \\
\hline 214. Knowledge of the guidelines for caloric intake for an individual desiring to lose or gain weight* & 4.21 & 90 & 4.32 & 95 \\
\hline $\begin{array}{l}\text { 215. Knowledge of common nutritional ergogenic aids, the purported mechanism of action, and any risk and/or } \\
\text { benefit (e.g., carbohydrates, protein/amino acids, vitamins, minerals, sodium bicarbonate, creatine, bee pollen) }\end{array}$ & 3.84 & 80 & 3.53 & 60 \\
\hline
\end{tabular}
cycle abnormalities, and osteoporosis) 
Table 11 (continued)

\begin{tabular}{|c|c|c|c|c|}
\hline \multirow[b]{2}{*}{ Exercise science competencies related to nutrition and weight management } & \multicolumn{2}{|c|}{ Importance } & \multicolumn{2}{|c|}{ Relevance } \\
\hline & $M$ & $\%$ & $M$ & $\%$ \\
\hline $\begin{array}{l}\text { 217. Knowledge of the NIH Consensus statement regarding health risks of obesity, Nutrition for Physical Fitness } \\
\text { Position Paper of the American Dietetic Association, and the ACSM Position Stand on proper and improper } \\
\text { weight loss programs }\end{array}$ & 4.30 & 95 & 3.60 & 55 \\
\hline 218. Knowledge of NCEP II guidelines for lipid management & 3.37 & 45 & 2.89 & 15 \\
\hline
\end{tabular}

*Indicates a consensus of agreement was reached regarding both the importance and relevance of this competency. Each item had to meet the following criteria for consensus: (a) The item received a mean rating of at least four or higher in the areas of importance and relevance; and (b) the item received at least $75 \%$ of all individual ratings at the four level or higher. 
Table 12 Category X - Program Management and Administration

\begin{tabular}{|c|c|c|c|c|}
\hline \multirow[b]{2}{*}{ Exercise science competencies related to program management and administration } & \multicolumn{2}{|c|}{ Importance } & \multicolumn{2}{|c|}{ Relevance } \\
\hline & $M$ & $\%$ & $M$ & $\%$ \\
\hline 219. Knowledge, skills, and ability to administer and deliver health/fitness programs* & 4.58 & 90 & 4.40 & 95 \\
\hline $\begin{array}{l}\text { 220. Ability to describe and use the documentation required when a client shows signs or symptoms during an } \\
\text { exercise session and should be referred to a physician }\end{array}$ & 3.84 & 80 & 3.32 & 40 \\
\hline 221. Ability to create and maintain records pertaining to participant exercise adherence, retention, and goal setting & 4.05 & 85 & 3.85 & 75 \\
\hline 222. Ability to develop and administer educational programs (e.g., lectures, workshops) and educational $n$ & 4.16 & 85 & 4.05 & 85 \\
\hline
\end{tabular}

*Indicates a consensus of agreement was reached regarding both the importance and relevance of this competency. Each item had to meet the following criteria for consensus: (a) The item received a mean rating of at least four or higher in the areas of importance and relevance; and (b) the item received at least $75 \%$ of all individual ratings at the four level or higher. 


\section{Table 13 Final List of Critical Exercise Science Competencies}

\section{Critical exercise science competencies}

1. Knowledge of anatomy as it relates to exercise and health

2. Knowledge of biomechanics as it relates to team/individual sports

3. Knowledge of the basic anatomy of the cardiovascular system and respiratory system

4. Knowledge of the biomechanical aspects of exercise participation

5. Knowledge of and skill to demonstrate exercises designed to enhance muscular strength and/or endurance of specific major muscle groups

6. Knowledge of and skill to demonstrate exercise for enhancing musculoskeletal flexibility

7. Knowledge of functional anatomy and biomechanics

8. Ability to locate common sites for measurement of skinfold thickness and circumferences (for determination of body composition and waist-hip ratio)

9. Knowledge of the biomechanical principles that underlie performance of the following activities: walking, jogging, running, swimming, cycling, weight lifting, and carrying or moving objects

10. Knowledge of the influence of weight-bearing activity in childhood and adolescence on optimal bone growth and development

11. Basic knowledge of exercise physiology as it relates to exercise prescription

12. Ability to define aerobic and anaerobic metabolism

13. Knowledge of the role of aerobic and anaerobic energy systems in the performance of various activities

14. Knowledge of the role of carbohydrates, fats, and proteins as fuels for aerobic and anaerobic metabolism

15. Knowledge of the components of fitness: cardiorespiratory fitness, muscular strength, muscular endurance, flexibility, and body composition

16. Knowledge to describe normal cardiorespiratory responses to static and dynamic exercise in terms of heart rate, blood pressure, and oxygen consumption

17. Knowledge of how heart rate, blood pressure, and oxygen consumption responses change with adaptation to chronic exercise training

18. Knowledge of the physiological adaptations associated with strength training

19. Ability to identify and apply to both groups and individuals methods used to monitor exercise intensity, including heart rate and rating of perceived exertion

20. Knowledge of the physiological principles related to warm-up and cool-down

21. Knowledge of exercise physiology including the role of aerobic and anaerobic metabolism, muscle physiology, cardiovascular physiology, and respiratory physiology at rest and during exercise. In addition, demonstrate an understanding of the components of physical fitness, the effects of aerobic and strength and/or resistance training on the fitness components and the effects of chronic disease

22. Ability to discuss the physiological principles involved in promoting gains in muscular strength and endurance

23. Ability to define muscular fatigue as it relates to task, intensity, duration, and the accumulative effects of exercise

24. Knowledge of the relationship between the number of repetitions, intensity, number of sets, and rest with regard to strength training 


\section{Table 13 (continued)}

Critical exercise science competencies

25. Knowledge of and ability to discuss the physiological basis of the major components of physical fitness: flexibility, cardiovascular fitness, muscular strength/endurance, and body composition

26. Ability to explain how the principle of specificity relates to the components of fitness

27. Ability to explain the concept of detraining or reversibility and its implications in fitness programs

28. Knowledge of the benefits and risks associated with exercise training in prepubescent and postpubescent youth

29. Knowledge of the changes that occur during growth and development from childhood to old age

30. Ability to modify cardiovascular and resistance exercises based on age and physical condition

31. Ability to characterize the differences in the development of an exercise prescription for children, adolescents, and older participants

32. Knowledge of and ability to describe the unique adaptations to exercise training in children, adolescents, and older participants with regard to strength, functional capacity, and motor skills

33. Knowledge of the health-related benefits of regular physical activity for people of all ages

34. Ability to determine those risk factors that may be favorably modified by physical activity habits

35. Knowledge of the risk factor concept of coronary artery disease (CAD) and the influence of heredity and lifestyle on the development of CAD

36. Ability to discuss in detail how lifestyle factors, including nutrition, physical activity, and heredity, influence lipid and lipoprotein profiles

37. Ability to identify and define at least five behavioral strategies to enhance exercise and health behavior change (i.e., reinforcement, goal setting, social support)

38. Knowledge of specific techniques to enhance motivation (e.g., posters, recognition, bulletin boards, games, competitions). Define extrinsic and intrinsic reinforcement and give examples of each

39. Ability to facilitate the development of positive attitudes and perceptions regarding physical activity and exercise

40. Knowledge of the use of goal setting and behavioral contracts as key components of the behavior change process

41. Knowledge of the environmental determinants of physical activity (e.g., television/computers, access to exercise facilities, neighborhood safety)

42. Knowledge of basic behavioral principles as related to classroom management and establishment of a safe, exercise environment

43. Knowledge of various educational theories and instructional methods that can be used in the area of physical activity promotion

44. Knowledge of the psychological benefits associated with participation in regular physical activity

45. Knowledge of the signs/symptoms of disordered eating and the appropriate procedures for referral

46. Skill to measure pulse rate accurately both at rest and during exercise

47. Knowledge, skills, and abilities to assess the health status of individuals and the ability to conduct fitness testing

48. Skill in instructing participants in the use of equipment and test procedures

49. Ability to locate and measure skinfold sites, skeletal diameters, and girth measurements used for estimating body composition 


\section{Table 13 (continued)}

\section{Critical exercise science competencies}

50. Ability to describe the purpose of testing, select appropriate protocols, and conduct assessments of muscular strength, muscular endurance, and flexibility

51. Ability to modify protocols and procedures for cardiorespiratory fitness tests in children, adolescents, and older adults

52. Knowledge of the steps needed to prepare children for physical fitness testing (e.g., teaching test purpose, protocol, pacing)

53. Knowledge of the differences between norm and criterion referenced fitness tests

54. Knowledge of the role of physical fitness testing in achieving national and state standards for physical education

55. Ability to use heart rate monitors for monitoring intensity during exercise

56. Knowledge of the NIH definitions of obesity and overweight using body mass index (BMI)

57. Ability to use pedometers for monitoring physical activity participation

58. Knowledge of and skill in obtaining basic life support and cardiopulmonary certification

59. Knowledge of appropriate emergency procedures (i.e., telephone procedures, written emergency procedures, personnel responsibilities) in the group exercise setting

60. Knowledge of basic first aid procedures for exercise-related injuries, such as bleeding, strains/sprains, fractures, and exercise intolerance (dizziness, syncope, heat injury)

61. Knowledge of basic precautions taken in a group exercise setting to ensure participant safety

62. Ability to list the effects of temperature, humidity, altitude, and pollution on physiological response to exercise

63. Knowledge of hypothetical concerns and potential risks that may be associated with the use of exercises such as straight leg sit-ups, double leg raises, full squats, hurdlers stretch, yoga plough, forceful back hyperextension, and standing bent-over toe touch

64. Knowledge of safety plans, emergency procedures, and first aid techniques needed during fitness evaluations, exercise testing, and exercise training

65. Ability to identify the components that contribute to the maintenance of a safe environment

66. Knowledge of the health/fitness instructor's responsibilities, limitations, and the legal implications of carrying out emergency procedures

67. Knowledge of the initial management and first aid techniques associated with open wounds, musculoskeletal injuries, cardiovascular/pulmonary complications, and metabolic disorders

68. Knowledge of the recommended intensity, duration, frequency, and type of physical activity necessary for development of cardiorespiratory fitness in an apparently healthy population

69. Ability to differentiate between the amount of physical activity required for health benefits and the amount of exercise required for fitness development

70. Ability to describe exercises designed to enhance muscular strength and/or endurance of specific major muscle groups

71. Knowledge of the principles of overload, specificity, and progression and how they relate to exercise programming

72. Skill to teach and demonstrate appropriate exercises used in the warm-up and cool-down of a variety of group exercise classes 
Table 13 (continued)

73. Ability to teach the components of an exercise session (i.e., warm-up, aerobic stimulus phase, cool-down, muscular strength/endurance, flexibility)

74. Knowledge of the following terms: progressive resistance, isotonic/isometric, concentric, eccentric, atrophy, hypertrophy, sets, repetitions, plyometrics, Valsalva maneuver

75. Skill to teach class participants how to monitor intensity of exercise using heart rate and rating of perceived exertion (RPE)

76. Skill to teach participants how to use RPE and heart rate to adjust the intensity of the exercise session

77. Ability to calculate training heart rates using two methods: percent of age-predicted maximum heart rate and heart rate reserve (Karvonen)

78. Ability to recognize proper and improper techniques in the use of resistive equipment such a stability balls, weights, bands, resistance bars, and water exercise equipment

79. Ability to recognize proper and improper technique in the use of cardiovascular conditioning equipment (e.g., steps, cycles, slides)

80. Skill to teach and demonstrate appropriate exercises for improving range of motion of major joints

81. Ability to modify exercises in group settings for apparently healthy persons of various fitness levels

82. Ability to teach a progression of exercises for all major muscle groups to improve muscular strength and endurance

83. Knowledge to describe the various types of interval, continuous, and circuit training programs

84. Ability to communicate effectively with exercise participants in the group exercise session

85. Knowledge to describe partner resistance exercises that can be used in a group class setting

86. Ability to demonstrate techniques for accommodating various fitness levels within the same class

87. Skill to demonstrate the proper form, alignment, and technique in typical exercises used in warm-up, stimulus, muscle conditioning, and cool-down phases of the group session

88. Ability to evaluate specific exercises in terms of safety and effectiveness for various participants

89. Ability to demonstrate a familiarity with a variety of group exercise formats (e.g., traditional, step, slide, muscle conditioning, flexibility, indoor cycling, water fitness, walking)

90. Ability to modify exercises based on age and physical condition

91. Ability to understand the components incorporated into an exercise session and the proper sequence (i.e., preexercise evaluation, warm-up, aerobic stimulus phase, cool-down, muscular strength and/or endurance, and flexibility)

92. Ability to design resistive exercise programs to increase or maintain muscular strength and/or endurance

93. Ability to evaluate flexibility and prescribe appropriate flexibility exercises for all major muscle groups

94. Knowledge of the importance of recording exercise sessions and performing periodic evaluations to assess changes in fitness status

95. Ability to design training programs using interval, continuous, and circuit training programs

96. Ability to include participants with different individual needs into a group exercise environment

97. Knowledge of the special concerns and guidelines recommended for children and adolescent strength training 


\section{Table 13 (continued)}

\section{Critical exercise science competencies}

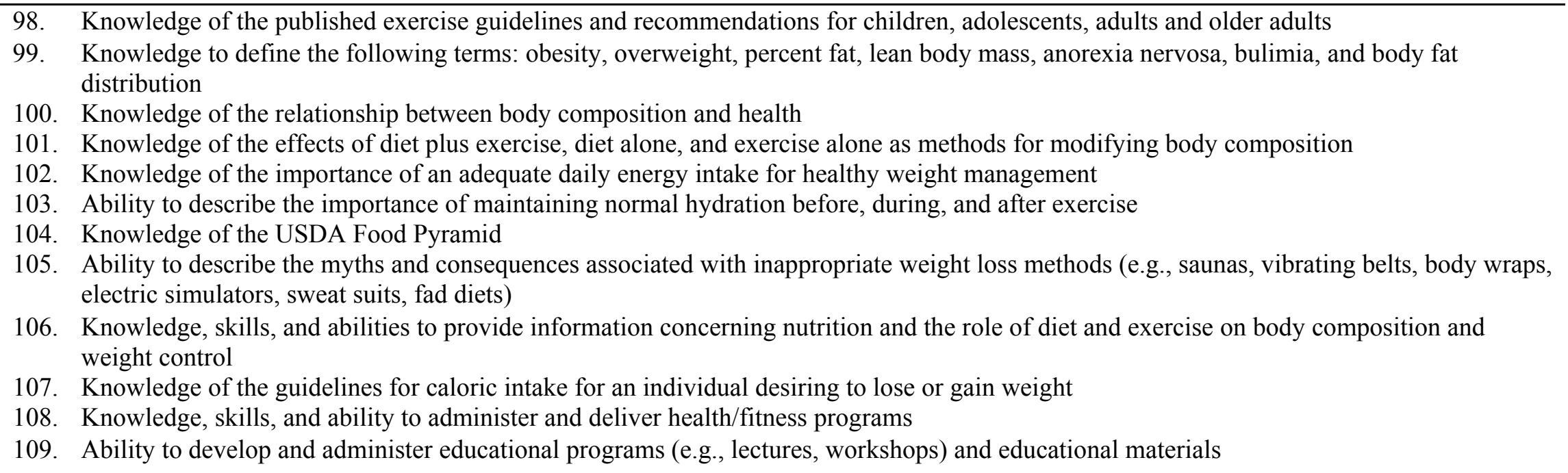

*Each item had to meet the following criteria for consensus: (a) The item received a mean rating of at least four or higher in the areas of importance and relevance; and (b) the item received at least $75 \%$ of all individual ratings at the four level or higher. 
Table 14 Delphi Panel Member Responses Regarding Non-Critical Exercise Science Competencies

\begin{tabular}{|c|c|c|c|c|c|c|c|c|}
\hline \multirow[b]{3}{*}{ Exercise Science Competencies } & \multicolumn{2}{|c|}{$\begin{array}{l}\text { Exercise Science } \\
\text { Specialists }\end{array}$} & \multicolumn{2}{|c|}{$\begin{array}{l}\text { Physical education } \\
\text { teacher educators }\end{array}$} & \multicolumn{2}{|c|}{$\begin{array}{l}\text { Physical education } \\
\text { teachers }\end{array}$} & \multicolumn{2}{|c|}{$\begin{array}{l}\text { Total Delphi panel } \\
\text { responses }\end{array}$} \\
\hline & Importance & Relevance & Importance & Relevance & Importance & Relevance & Importance & Relevance \\
\hline & $M$ & $M$ & $M$ & $M$ & $M$ & $M$ & $S D$ & $S D$ \\
\hline $\begin{array}{l}\text { 2. Knowledge of anatomy as it relates to } \\
\text { team/individual sports }\end{array}$ & 3.90 & 3.90 & 4.14 & 4.14 & 4.00 & 3.67 & 0.56 & 0.60 \\
\hline $\begin{array}{l}\text { 4. Knowledge of the basic structures of } \\
\text { bone, skeletal muscle, and connective } \\
\text { tissues }\end{array}$ & 4.40 & 3.60 & 4.57 & 4.29 & 4.33 & 4.33 & 0.60 & 0.60 \\
\hline 6. Ability to identify the major bones & 4.40 & 3.70 & 4.71 & 3.86 & 5.00 & 4.33 & 0.60 & 0.81 \\
\hline $\begin{array}{l}\text { 7. Knowledge of the definition of the terms: } \\
\text { supination, pronation, flexion, and so forth }\end{array}$ & 4.10 & 3.60 & 4.57 & 3.43 & 4.67 & 3.67 & 0.67 & 0.83 \\
\hline 8. Ability to identify the joints of the body & 4.00 & 3.40 & 4.43 & 3.57 & 5.00 & 4.00 & 0.66 & 0.89 \\
\hline $\begin{array}{l}\text { 10. Knowledge to identify the plane in } \\
\text { which each muscle action occurs }\end{array}$ & 3.40 & 2.70 & 3.33 & 2.33 & 3.33 & 2.67 & 1.01 & 0.77 \\
\hline $\begin{array}{l}\text { 11. Knowledge of the interrelationships } \\
\text { among center of gravity, base of support, } \\
\text { balance, stability, and spinal alignment }\end{array}$ & 4.00 & 3.50 & 4.29 & 4.00 & 4.33 & 4.00 & 0.75 & 0.72 \\
\hline $\begin{array}{l}\text { 12. Ability to describe the curvatures of the } \\
\text { spine: lordosis, scoliosis, and kyphosis }\end{array}$ & 3.70 & 3.40 & 3.71 & 3.14 & 3.33 & 3.33 & 0.93 & 1.03 \\
\hline $\begin{array}{l}\text { 15. Knowledge to describe the myotatic } \\
\text { stretch reflex }\end{array}$ & 3.50 & 2.80 & 3.33 & 2.67 & 3.33 & 2.67 & 1.12 & 1.05 \\
\hline $\begin{array}{l}\text { 16. Knowledge to identify the primary } \\
\text { action and joint range of motion for each } \\
\text { major muscle group }\end{array}$ & 3.90 & 3.60 & 4.14 & 3.71 & 4.00 & 3.67 & 0.79 & 0.75 \\
\hline $\begin{array}{l}\text { 18. Knowledge of the structure and ability } \\
\text { to describe movements for the major joints } \\
\text { of the body }\end{array}$ & 4.20 & 3.90 & 4.43 & 4.00 & 4.33 & 4.00 & 0.57 & 0.60 \\
\hline $\begin{array}{l}\text { 19. Ability to locate the anatomic landmarks } \\
\text { for palpation of peripheral pulses }\end{array}$ & 3.70 & 3.30 & 4.00 & 4.00 & 4.33 & 4.00 & 0.94 & 0.83 \\
\hline $\begin{array}{l}\text { 20. Ability to locate the brachial artery and } \\
\text { correctly place the cuff and stethoscope in } \\
\text { position for blood pressure measurement }\end{array}$ & 4.00 & 3.50 & 3.71 & 3.17 & 3.33 & 3.00 & 0.77 & 0.75 \\
\hline
\end{tabular}




\begin{tabular}{|c|c|c|c|c|c|c|c|c|}
\hline \multirow[b]{3}{*}{ Exercise Science Competencies } & \multicolumn{2}{|c|}{$\begin{array}{l}\text { Exercise Science } \\
\text { Specialists }\end{array}$} & \multicolumn{2}{|c|}{$\begin{array}{l}\text { Physical education } \\
\text { teacher educators }\end{array}$} & \multicolumn{2}{|c|}{$\begin{array}{l}\text { Physical education } \\
\text { teachers }\end{array}$} & \multicolumn{2}{|c|}{$\begin{array}{l}\text { Total Delphi panel } \\
\text { responses }\end{array}$} \\
\hline & Importance & Relevance & Importance & Relevance & Importance & Relevance & Importance & Relevance \\
\hline & $M$ & $M$ & $M$ & $M$ & $M$ & $M$ & $S D$ & $S D$ \\
\hline $\begin{array}{l}\text { 27. Knowledge of the following terms: } \\
\text { ischemia, angina pectoris, tachycardia, and } \\
\text { so forth }\end{array}$ & 4.30 & 3.40 & 4.29 & 3.71 & 3.67 & 3.00 & 0.62 & 0.69 \\
\hline $\begin{array}{l}\text { 35. Knowledge of the common theories of } \\
\text { muscle fatigue and delayed onset muscle } \\
\text { soreness (DOMS) }\end{array}$ & 4.10 & 3.90 & 4.29 & 3.71 & 4.33 & 4.00 & 0.83 & 0.93 \\
\hline $\begin{array}{l}\text { 37. Knowledge of the physiological } \\
\text { adaptations that occur at rest and during } \\
\text { submaximal and maximal exercise } \\
\text { following chronic aerobic/anaerobic } \\
\text { exercise training }\end{array}$ & 4.30 & 4.10 & 4.43 & 3.86 & 4.00 & 3.67 & 0.47 & 0.69 \\
\hline $\begin{array}{l}\text { 38. Knowledge of the differences in } \\
\text { cardiorespiratory response to acute graded } \\
\text { exercise between conditioned and } \\
\text { unconditioned individuals }\end{array}$ & 4.20 & 3.44 & 4.14 & 3.57 & 3.33 & 3.00 & 0.51 & 0.61 \\
\hline $\begin{array}{l}\text { 39. Knowledge of the structure of the } \\
\text { skeletal muscle fiber and the basic } \\
\text { mechanism of contraction }\end{array}$ & 3.90 & 3.20 & 4.00 & 3.00 & 4.67 & 4.00 & 0.83 & 0.97 \\
\hline $\begin{array}{l}\text { 40. Knowledge of the characteristics of fast } \\
\text { and slow twitch fibers }\end{array}$ & 3.80 & 3.50 & 4.00 & 3.00 & 4.00 & 4.00 & 0.99 & 0.98 \\
\hline $\begin{array}{l}\text { 41. Knowledge of the sliding filament } \\
\text { theory of muscle contraction }\end{array}$ & 3.50 & 2.80 & 3.50 & 2.33 & 3.33 & 3.67 & 1.07 & 0.96 \\
\hline $\begin{array}{l}\text { 42. Knowledge of twitch, summation, and } \\
\text { tetanus with respect to muscle contraction }\end{array}$ & 3.40 & 2.50 & 3.17 & 2.17 & 3.33 & 2.67 & 1.00 & 0.84 \\
\hline $\begin{array}{l}\text { 46. Knowledge of the basic properties of } \\
\text { cardiac muscle and normal pathways of } \\
\text { conduction in heart }\end{array}$ & 3.70 & 2.60 & 3.71 & 2.43 & 3.67 & 3.00 & 0.86 & 0.88 \\
\hline $\begin{array}{l}\text { 47. Knowledge of the response of these } \\
\text { variables to acute exercise: heart rate, stroke } \\
\text { volume, cardiac output, and so forth }\end{array}$ & 4.00 & 3.50 & 4.00 & 3.57 & 3.33 & 3.00 & 0.55 & 0.76 \\
\hline
\end{tabular}




\begin{tabular}{|c|c|c|c|c|c|c|c|c|}
\hline \multirow[b]{3}{*}{ Exercise Science Competencies } & \multicolumn{2}{|c|}{$\begin{array}{l}\text { Exercise Science } \\
\text { Specialists }\end{array}$} & \multicolumn{2}{|c|}{$\begin{array}{l}\text { Physical education } \\
\text { teacher educators }\end{array}$} & \multicolumn{2}{|c|}{$\begin{array}{l}\text { Physical education } \\
\text { teachers }\end{array}$} & \multicolumn{2}{|c|}{$\begin{array}{l}\text { Total Delphi panel } \\
\text { responses }\end{array}$} \\
\hline & Importance & Relevance & Importance & Relevance & Importance & Relevance & Importance & Relevance \\
\hline & $M$ & $M$ & $M$ & $M$ & $M$ & $M$ & $S D$ & $S D$ \\
\hline $\begin{array}{l}\text { 48. Knowledge of the differences in the } \\
\text { cardiorespiratory responses to static } \\
\text { exercise compared with dynamic exercise, } \\
\text { including possible hazards and } \\
\text { contraindications }\end{array}$ & 3.70 & 3.90 & 4.00 & 3.86 & 4.00 & 4.00 & 0.75 & 0.64 \\
\hline $\begin{array}{l}\text { 49. Ability to describe how each of the } \\
\text { following differs from the normal condition: } \\
\text { premature atrial contractions and premature } \\
\text { ventricular contractions }\end{array}$ & 2.80 & 2.30 & 2.86 & 2.14 & 3.00 & 2.67 & 0.99 & 0.86 \\
\hline $\begin{array}{l}\text { 50. Knowledge of blood pressure responses } \\
\text { associated with acute exercise, including } \\
\text { changes in body position }\end{array}$ & 3.90 & 3.30 & 3.71 & 3.43 & 4.33 & 3.67 & 0.64 & 0.75 \\
\hline $\begin{array}{l}\text { 51. Knowledge of and ability to describe the } \\
\text { implications of ventilatory threshold } \\
\text { (anaerobic threshold) as it relates to } \\
\text { exercise training and cardiorespiratory } \\
\text { assessment }\end{array}$ & 4.10 & 3.50 & 4.00 & 3.33 & 3.67 & 3.33 & 0.58 & 0.77 \\
\hline $\begin{array}{l}\text { 52. Knowledge of and ability to describe the } \\
\text { physiological adaptations of the respiratory } \\
\text { system that occur at rest and during } \\
\text { submaximal/maximal exercise following } \\
\text { chronic aerobic/anaerobic training }\end{array}$ & 4.00 & 3.50 & 4.29 & 3.57 & 4.00 & 3.67 & 0.72 & 0.94 \\
\hline $\begin{array}{l}\text { 53. Ability to describe how each of the } \\
\text { following differs from the normal condition: } \\
\text { dyspnea, hypoxia, and hypoventilation }\end{array}$ & 3.30 & 2.60 & 3.00 & 2.17 & 2.67 & 2.33 & 0.97 & 0.90 \\
\hline $\begin{array}{l}\text { 57. Ability to discuss the physical and } \\
\text { psychological signs of overtraining and } \\
\text { provide recommendations for these } \\
\text { problems }\end{array}$ & 4.00 & 3.90 & 4.29 & 3.71 & 4.33 & 3.33 & 0.75 & 0.91 \\
\hline $\begin{array}{l}\text { 58. Ability to describe the physiological and } \\
\text { metabolic responses to exercise associated } \\
\text { with chronic disease }\end{array}$ & 3.70 & 3.50 & 3.86 & 3.43 & 3.67 & 3.33 & 0.91 & 0.89 \\
\hline
\end{tabular}




\begin{tabular}{|c|c|c|c|c|c|c|c|c|}
\hline \multirow[b]{3}{*}{ Exercise Science Competencies } & \multicolumn{2}{|c|}{$\begin{array}{c}\text { Exercise Science } \\
\text { Specialists }\end{array}$} & \multicolumn{2}{|c|}{$\begin{array}{l}\text { Physical education } \\
\text { teacher educators }\end{array}$} & \multicolumn{2}{|c|}{$\begin{array}{l}\text { Physical education } \\
\text { teachers }\end{array}$} & \multicolumn{2}{|c|}{$\begin{array}{l}\text { Total Delphi panel } \\
\text { responses }\end{array}$} \\
\hline & Importance & Relevance & Importance & Relevance & Importance & Relevance & Importance & Relevance \\
\hline & $M$ & $M$ & $M$ & $M$ & $M$ & $M$ & $S D$ & $S D$ \\
\hline $\begin{array}{l}\text { 60. Knowledge of the benefits and } \\
\text { precautions associated with resistance and } \\
\text { endurance training in older adults }\end{array}$ & 3.89 & 2.89 & 3.43 & 2.14 & 3.33 & 2.33 & 0.96 & 0.96 \\
\hline $\begin{array}{l}\text { 61. Ability to describe specific leadership } \\
\text { techniques appropriate for working with } \\
\text { participants of all ages }\end{array}$ & 3.75 & 3.38 & 4.14 & 4.00 & 4.67 & 4.33 & 0.87 & 0.94 \\
\hline $\begin{array}{l}\text { 64. Knowledge of and ability to describe the } \\
\text { changes that occur in maturation from } \\
\text { childhood to adulthood: skeletal muscle, } \\
\text { bone structure, reaction time, coordination, } \\
\text { heat and cold tolerance, maximal oxygen } \\
\text { consumption, strength, flexibility, body } \\
\text { composition, resting and maximal heart } \\
\text { rate, and resting and maximal blood } \\
\text { pressure }\end{array}$ & 4.33 & 3.89 & 4.57 & 4.14 & 4.67 & 3.67 & 0.51 & 0.85 \\
\hline $\begin{array}{l}\text { 65. Knowledge of the effect of the aging } \\
\text { process on the musculoskeletal and } \\
\text { cardiovascular structure and function at rest, } \\
\text { during exercise, and during recovery }\end{array}$ & 3.22 & 2.67 & 3.57 & 3.00 & 4.00 & 3.33 & 0.90 & 0.88 \\
\hline $\begin{array}{l}\text { 68. Knowledge of the common orthopedic } \\
\text { and cardiovascular considerations for older } \\
\text { participants and the ability to describe } \\
\text { modifications in exercise prescription that } \\
\text { are indicated }\end{array}$ & 2.90 & 2.50 & 2.83 & 2.20 & 2.67 & 2.00 & 0.83 & 0.77 \\
\hline $\begin{array}{l}\text { 70. Knowledge of cardiovascular, } \\
\text { respiratory, metabolic, and musculoskeletal } \\
\text { risk factors that may require further } \\
\text { evaluation by medical or allied health } \\
\text { professionals before participation in } \\
\text { physical activity }\end{array}$ & 4.00 & 3.60 & 4.29 & 3.71 & 3.33 & 2.67 & 0.73 & 0.89 \\
\hline
\end{tabular}




\begin{tabular}{|c|c|c|c|c|c|c|c|c|}
\hline \multirow[b]{3}{*}{ Exercise Science Competencies } & \multicolumn{2}{|c|}{$\begin{array}{c}\text { Exercise Science } \\
\text { Specialists }\end{array}$} & \multicolumn{2}{|c|}{$\begin{array}{l}\text { Physical education } \\
\text { teacher educators }\end{array}$} & \multicolumn{2}{|c|}{$\begin{array}{c}\text { Physical education } \\
\text { teachers }\end{array}$} & \multicolumn{2}{|c|}{$\begin{array}{l}\text { Total Delphi panel } \\
\text { responses }\end{array}$} \\
\hline & Importance & Relevance & Importance & Relevance & Importance & Relevance & Importance & Relevance \\
\hline & $M$ & $M$ & $M$ & $M$ & $M$ & $M$ & $S D$ & $S D$ \\
\hline $\begin{array}{l}\text { 72. Knowledge to define the following } \\
\text { terms: total cholesterol (TC), high-density } \\
\text { lipoprotein cholesterol (HDL-C), TC/HDL- } \\
\text { C ratio, low-density lipoprotein cholesterol } \\
\text { (LDL-C), triglycerides, hypertension, and } \\
\text { artherosclerosis }\end{array}$ & 4.30 & 3.60 & 4.43 & 3.86 & 4.00 & 3.33 & 0.47 & 0.75 \\
\hline $\begin{array}{l}\text { 73. Knowledge of plasma cholesterol levels } \\
\text { for adults as recommended by the National } \\
\text { Cholesterol Education Program (NCEP II) }\end{array}$ & 3.70 & 3.30 & 3.00 & 2.33 & 2.33 & 2.33 & 0.99 & 0.76 \\
\hline $\begin{array}{l}\text { 74. Knowledge of the pathophysiology of } \\
\text { atherosclerosis and how this process is } \\
\text { influenced by physical activity }\end{array}$ & 4.10 & 3.75 & 3.71 & 3.29 & 4.33 & 3.67 & 0.73 & 0.86 \\
\hline $\begin{array}{l}\text { 76. Knowledge of the artherosclerotic } \\
\text { process, the factors involved in its genesis } \\
\text { and progression, and the potential role of } \\
\text { exercise training in treatment }\end{array}$ & 3.90 & 3.40 & 3.71 & 2.86 & 3.00 & 2.00 & 0.92 & 1.03 \\
\hline $\begin{array}{l}\text { 78. Knowledge of cardiovascular risk } \\
\text { factors or conditions that may require } \\
\text { consultation with medical personnel before } \\
\text { testing or training, including inappropriate } \\
\text { changes in resting or exercise heart rate and } \\
\text { blood pressure, new onset discomfort in } \\
\text { chest, neck, shoulder, or arm, and so forth }\end{array}$ & 4.10 & 3.80 & 4.14 & 3.71 & 4.67 & 3.67 & 0.62 & 0.72 \\
\hline $\begin{array}{l}\text { 79. Knowledge of the respiratory risk } \\
\text { factors or conditions that may require } \\
\text { consultation with medical personnel before } \\
\text { testing or training, including asthma, } \\
\text { exercise-induced bronchospasm, extreme } \\
\text { breathlessness at rest or during exercise, } \\
\text { bronchitis, emphysema }\end{array}$ & 4.00 & 3.90 & 4.43 & 4.00 & 4.00 & 3.67 & 0.49 & 0.64 \\
\hline
\end{tabular}




\begin{tabular}{|c|c|c|c|c|c|c|c|c|}
\hline \multirow[b]{3}{*}{ Exercise Science Competencies } & \multicolumn{2}{|c|}{$\begin{array}{l}\text { Exercise Science } \\
\text { Specialists }\end{array}$} & \multicolumn{2}{|c|}{$\begin{array}{l}\text { Physical education } \\
\text { teacher educators }\end{array}$} & \multicolumn{2}{|c|}{$\begin{array}{l}\text { Physical education } \\
\text { teachers }\end{array}$} & \multicolumn{2}{|c|}{$\begin{array}{l}\text { Total Delphi panel } \\
\text { responses }\end{array}$} \\
\hline & Importance & Relevance & Importance & Relevance & Importance & Relevance & Importance & Relevance \\
\hline & $M$ & $M$ & $M$ & $M$ & $M$ & $M$ & $S D$ & $S D$ \\
\hline $\begin{array}{l}80 . \text { Knowledge of the metabolic risk factors } \\
\text { or conditions that may require consultation } \\
\text { with medical personnel before testing or } \\
\text { training, including bodyweight more than } \\
20 \% \text { above optimal, BMI }>30 \text {, thyroid } \\
\text { disease, diabetes or glucose intolerance, and } \\
\text { hypoglycemia }\end{array}$ & 4.00 & 3.90 & 4.00 & 4.00 & 3.67 & 3.33 & 0.69 & 0.76 \\
\hline $\begin{array}{l}\text { 81. Knowledge of the musculoskeletal risk } \\
\text { factors or conditions that may require } \\
\text { consultation with medical personnel before } \\
\text { testing or training, including acute or } \\
\text { chronic back pain, osteoarthritis, } \\
\text { rheumatoid arthritis, osteoporosis, } \\
\text { tendonitis, and low back pain }\end{array}$ & 4.00 & 3.40 & 3.86 & 2.86 & 3.67 & 3.33 & 0.55 & 0.77 \\
\hline $\begin{array}{l}\text { 82. Knowledge of persons with physical and } \\
\text { mental disabilities and the potential } \\
\text { influence on risk for chronic degenerative } \\
\text { disease }\end{array}$ & 3.60 & 3.10 & 4.00 & 3.50 & 4.00 & 3.33 & 0.85 & 0.99 \\
\hline $\begin{array}{l}\text { 83. Knowledge of the factors that increase } \\
\text { risk for osteoporosis and the preventive } \\
\text { measures that can be followed to decrease } \\
\text { risk }\end{array}$ & 4.00 & 3.56 & 3.57 & 2.33 & 4.00 & 3.33 & 0.67 & 0.83 \\
\hline $\begin{array}{l}\text { 85. Ability to list and define the five } \\
\text { important elements that should be included } \\
\text { in a health counseling session }\end{array}$ & 3.44 & 2.89 & 2.83 & 2.33 & 3.00 & 3.00 & 1.19 & 0.99 \\
\hline $\begin{array}{l}87 . \text { Knowledge of the stages of motivational } \\
\text { readiness }\end{array}$ & 3.80 & 3.78 & 3.50 & 3.17 & 4.00 & 4.00 & 0.89 & 1.06 \\
\hline 88. Ability to list and describe three & 3.90 & 3.56 & 3.00 & 2.83 & 3.00 & 3.00 & 0.99 & 0.97 \\
\hline
\end{tabular}

88. Ability to list and describe three

3.90

3.56

3.00

(.83

motivated clients to increase their physical

activity 


\begin{tabular}{|c|c|c|c|c|c|c|c|c|}
\hline \multirow{3}{*}{ Exercise Science Competencies } & \multicolumn{2}{|c|}{$\begin{array}{l}\text { Exercise Science } \\
\text { Specialists }\end{array}$} & \multicolumn{2}{|c|}{$\begin{array}{l}\text { Physical education } \\
\text { teacher educators }\end{array}$} & \multicolumn{2}{|c|}{$\begin{array}{l}\text { Physical education } \\
\text { teachers }\end{array}$} & \multicolumn{2}{|c|}{$\begin{array}{l}\text { Total Delphi panel } \\
\text { responses }\end{array}$} \\
\hline & Importance & Relevance & Importance & Relevance & Importance & Relevance & Importance & Relevance \\
\hline & $M$ & $M$ & $M$ & $M$ & $M$ & $M$ & $S D$ & $S D$ \\
\hline $\begin{array}{l}\text { 89. Ability to list and describe the specific } \\
\text { strategies aimed at encouraging initiation, } \\
\text { adherence, and return to participation in an } \\
\text { exercise program }\end{array}$ & 4.10 & 3.89 & 4.17 & 3.50 & 4.00 & 4.00 & 0.68 & 0.97 \\
\hline $\begin{array}{l}\text { 90. Knowledge of symptoms of anxiety and } \\
\text { depression that may necessitate referral }\end{array}$ & 3.44 & 2.75 & 3.17 & 2.83 & 4.50 & 4.00 & 0.94 & 1.18 \\
\hline $\begin{array}{l}\text { 91. Knowledge of the potential symptoms } \\
\text { and causal factors of test anxiety (i.e., } \\
\text { performance, appraisal threat during } \\
\text { exercise testing) and how it may affect } \\
\text { physiological responses to testing }\end{array}$ & 3.00 & 2.44 & 3.33 & 2.67 & 5.00 & 4.50 & 1.24 & 1.15 \\
\hline $\begin{array}{l}\text { 94. Knowledge of the psycho-social } \\
\text { determinants of physical activity (e.g., } \\
\text { parental/peer influence, self-efficacy, self- } \\
\text { perception) }\end{array}$ & 4.00 & 3.40 & 4.14 & 4.00 & 4.67 & 4.67 & 0.88 & 1.01 \\
\hline $\begin{array}{l}\text { 99. Knowledge of the psychological } \\
\text { variables associated with sport performance } \\
\text { (e.g., anxiety, motivation, burnout) }\end{array}$ & 3.60 & 3.30 & 3.86 & 3.43 & 4.00 & 4.00 & 0.79 & 1.00 \\
\hline $\begin{array}{l}\text { 101. Knowledge of the importance of a } \\
\text { health/medical history }\end{array}$ & 3.90 & 3.60 & 4.29 & 3.86 & 4.00 & 3.67 & 0.60 & 0.80 \\
\hline $\begin{array}{l}\text { 102. Knowledge of the value of a medical } \\
\text { clearance prior to exercise participation }\end{array}$ & 4.20 & 3.78 & 4.43 & 4.00 & 4.33 & 4.33 & 0.47 & 0.91 \\
\hline $\begin{array}{l}\text { 105. Ability to obtain a health history and } \\
\text { risk appraisal that includes past and current } \\
\text { medical history, family history of cardiac } \\
\text { disease, orthopedic limitations, prescribed } \\
\text { medications, activity patterns, nutritional } \\
\text { habits, stress and anxiety levels, and } \\
\text { smoking and alcohol use }\end{array}$ & 3.50 & 3.00 & 3.14 & 2.43 & 2.33 & 1.67 & 0.95 & 1.05 \\
\hline
\end{tabular}




\begin{tabular}{|c|c|c|c|c|c|c|c|c|}
\hline \multirow[b]{3}{*}{ Exercise Science Competencies } & \multicolumn{2}{|c|}{$\begin{array}{c}\text { Exercise Science } \\
\text { Specialists }\end{array}$} & \multicolumn{2}{|c|}{$\begin{array}{l}\text { Physical education } \\
\text { teacher educators }\end{array}$} & \multicolumn{2}{|c|}{$\begin{array}{l}\text { Physical education } \\
\text { teachers }\end{array}$} & \multicolumn{2}{|c|}{$\begin{array}{l}\text { Total Delphi panel } \\
\text { responses }\end{array}$} \\
\hline & Importance & Relevance & Importance & Relevance & Importance & Relevance & Importance & Relevance \\
\hline & $M$ & $M$ & $M$ & $M$ & $M$ & $M$ & $S D$ & $S D$ \\
\hline $\begin{array}{l}\text { 106. Ability to describe the categories of } \\
\text { participants who should receive medical } \\
\text { clearance prior to administration of an } \\
\text { exercise test or participation in an exercise } \\
\text { program }\end{array}$ & 3.60 & 3.10 & 3.71 & 3.29 & 3.67 & 2.67 & 0.93 & 1.02 \\
\hline $\begin{array}{l}\text { 107. Ability to identify relative and absolute } \\
\text { contraindications to exercise testing or } \\
\text { participation }\end{array}$ & 3.70 & 3.60 & 4.00 & 3.57 & 3.33 & 3.33 & 0.79 & 1.00 \\
\hline $\begin{array}{l}\text { 108. Ability to discuss the limitations of } \\
\text { informed consent and medical clearance } \\
\text { prior to exercise testing }\end{array}$ & 3.90 & 2.90 & 3.43 & 2.43 & 3.33 & 3.00 & 0.99 & 1.02 \\
\hline 109. Ability to obtain informed consent & 3.89 & 3.44 & 3.86 & 3.29 & 3.67 & 2.67 & 0.83 & 1.15 \\
\hline $\begin{array}{l}\text { 110. Ability to explain the purpose and } \\
\text { procedures for monitoring clients prior to, } \\
\text { during, and after cardiorespiratory fitness } \\
\text { testing }\end{array}$ & 3.80 & 3.30 & 4.00 & 3.14 & 3.67 & 3.67 & 0.75 & 0.98 \\
\hline $\begin{array}{l}\text { 112. Ability to describe the purpose of } \\
\text { testing, select an appropriate submaximal or } \\
\text { maximal protocol, and conduct an } \\
\text { assessment of cardiovascular fitness on the } \\
\text { cycle ergometer or the treadmill }\end{array}$ & 3.80 & 3.10 & 3.14 & 2.29 & 3.67 & 2.33 & 0.83 & 0.80 \\
\hline $\begin{array}{l}\text { 113. Skill in accurately measuring heart } \\
\text { rate, blood pressure, and obtaining rating of } \\
\text { perceived exertion (RPE) at rest and during } \\
\text { exercise according to established guidelines }\end{array}$ & 4.20 & 3.80 & 4.14 & 3.57 & 4.33 & 3.67 & 0.62 & 0.98 \\
\hline $\begin{array}{l}116 \text {. Skill in various techniques of assessing } \\
\text { body composition }\end{array}$ & 4.30 & 4.20 & 4.00 & 4.00 & 4.14 & 3.71 & 0.70 & 0.92 \\
\hline $\begin{array}{l}\text { 117. Knowledge of the advantages and } \\
\text { disadvantages and limitations of the various } \\
\text { body composition techniques }\end{array}$ & 4.30 & 3.50 & 4.43 & 3.71 & 4.67 & 4.33 & 0.75 & 1.03 \\
\hline
\end{tabular}




\begin{tabular}{|c|c|c|c|c|c|c|c|c|}
\hline \multirow[b]{3}{*}{ Exercise Science Competencies } & \multicolumn{2}{|c|}{$\begin{array}{c}\text { Exercise Science } \\
\text { Specialists }\end{array}$} & \multicolumn{2}{|c|}{$\begin{array}{l}\text { Physical education } \\
\text { teacher educators }\end{array}$} & \multicolumn{2}{|c|}{$\begin{array}{c}\text { Physical education } \\
\text { teachers }\end{array}$} & \multicolumn{2}{|c|}{$\begin{array}{l}\text { Total Delphi panel } \\
\text { responses }\end{array}$} \\
\hline & Importance & Relevance & Importance & Relevance & Importance & Relevance & Importance & Relevance \\
\hline & $M$ & $M$ & $M$ & $M$ & $M$ & $M$ & $S D$ & $S D$ \\
\hline $\begin{array}{l}\text { 118. Ability to interpret information } \\
\text { obtained from cardiorespiratory fitness tests } \\
\text { and muscular strength/endurance, } \\
\text { flexibility, and body composition tests for } \\
\text { apparently healthy individuals and those } \\
\text { with stable disease }\end{array}$ & 3.80 & 3.60 & 4.14 & 3.86 & 3.33 & 3.00 & 1.09 & 1.14 \\
\hline $\begin{array}{l}\text { 119. Ability to identify appropriate criteria } \\
\text { for terminating a fitness evaluation and } \\
\text { demonstrate proper procedures to be } \\
\text { followed after discontinuing such a test }\end{array}$ & 3.70 & 3.50 & 4.14 & 3.43 & 3.67 & 3.33 & 0.93 & 1.05 \\
\hline $\begin{array}{l}\text { 121. Ability to conduct fitness appraisals for } \\
\text { populations with physical and mental } \\
\text { disabilities (e.g., mental retardation) which } \\
\text { may require alternative testing techniques }\end{array}$ & 3.50 & 3.56 & 4.43 & 4.00 & 3.67 & 3.00 & 0.88 & 0.90 \\
\hline $\begin{array}{l}\text { 128. Knowledge of bronchodilators and } \\
\text { their principal effects on exercise testing } \\
\text { and prescription }\end{array}$ & 3.20 & 3.10 & 3.00 & 2.67 & 3.33 & 3.33 & 1.04 & 1.15 \\
\hline $\begin{array}{l}\text { 129. Knowledge of hypoglycemics and their } \\
\text { principal effects on exercise testing and } \\
\text { prescription }\end{array}$ & 3.20 & 2.90 & 3.00 & 2.67 & 2.67 & 2.33 & 0.89 & 0.93 \\
\hline $\begin{array}{l}\text { 130. Knowledge of psychotropics and their } \\
\text { principal effects on exercise testing and } \\
\text { prescription }\end{array}$ & 3.10 & 2.60 & 2.57 & 2.33 & 2.67 & 2.00 & 0.81 & 0.69 \\
\hline $\begin{array}{l}\text { 131. Knowledge of vasodilators and their } \\
\text { principal effects on exercise testing and } \\
\text { prescription }\end{array}$ & 2.90 & 2.60 & 2.71 & 2.17 & 3.33 & 3.00 & 0.91 & 0.96 \\
\hline 132. Ability to identify the effects of the & 3.10 & 2.90 & 3.71 & 3.57 & 3.00 & 2.33 & 0.92 & 0.89 \\
\hline
\end{tabular}

following substances on exercise response: antihistamines, tranquilizers, alcohol, diet pills, cold tablets, caffeine, and nicotine 


\begin{tabular}{|c|c|c|c|c|c|c|c|c|}
\hline \multirow{3}{*}{ Exercise Science Competencies } & \multicolumn{2}{|c|}{$\begin{array}{l}\text { Exercise Science } \\
\text { Specialists }\end{array}$} & \multicolumn{2}{|c|}{$\begin{array}{l}\text { Physical education } \\
\text { teacher educators }\end{array}$} & \multicolumn{2}{|c|}{$\begin{array}{l}\text { Physical education } \\
\text { teachers }\end{array}$} & \multicolumn{2}{|c|}{$\begin{array}{l}\text { Total Delphi panel } \\
\text { responses }\end{array}$} \\
\hline & Importance & Relevance & Importance & Relevance & Importance & Relevance & Importance & Relevance \\
\hline & $M$ & $M$ & $M$ & $M$ & $M$ & $M$ & $S D$ & $S D$ \\
\hline $\begin{array}{l}\text { 137. Ability to identify the physical and } \\
\text { physiological signs and symptoms of } \\
\text { overtraining }\end{array}$ & 3.80 & 3.40 & 4.29 & 3.71 & 4.33 & 4.00 & 0.94 & 1.10 \\
\hline $\begin{array}{l}\text { 139. Knowledge of the following terms: } \\
\text { shin splints, sprain, strain, tennis elbow, } \\
\text { bursitis, stress fracture, tendonitis, patellar } \\
\text { femoral pain syndrome, low back pain, } \\
\text { plantar fasciitis, and rotator cuff tendonitis }\end{array}$ & 4.20 & 3.60 & 4.57 & 3.71 & 4.33 & 4.33 & 0.67 & 1.02 \\
\hline $\begin{array}{l}\text { 140. Skill to demonstrate exercises used for } \\
\text { people with low back pain }\end{array}$ & 3.70 & 3.40 & 4.43 & 3.29 & 4.33 & 3.67 & 0.60 & 1.10 \\
\hline $\begin{array}{l}\text { 142. Skill in demonstrating appropriate } \\
\text { emergency procedures during exercise } \\
\text { testing and/or training }\end{array}$ & 4.00 & 3.33 & 4.43 & 4.00 & 4.00 & 4.00 & 0.69 & 0.89 \\
\hline $\begin{array}{l}\text { 146. Ability to describe potential } \\
\text { musculoskeletal injuries (e.g., contusions, } \\
\text { sprains, strains, fractures), cardiovascular/ } \\
\text { pulmonary complications (e.g., tachycardia, } \\
\text { bradycardia, tachypnea), and metabolic } \\
\text { abnormalities (e.g., fainting/syncope, } \\
\text { hypoglycemia/ hyperglycemia) }\end{array}$ & 3.90 & 3.70 & 3.43 & 3.00 & 3.33 & 3.67 & 0.88 & 1.00 \\
\hline $\begin{array}{l}\text { 148. Knowledge of the components of an } \\
\text { equipment maintenance/repair program and } \\
\text { how it may be used to evaluate the } \\
\text { condition of exercise equipment to reduce } \\
\text { potential risk }\end{array}$ & 3.50 & 3.60 & 3.29 & 2.43 & 4.67 & 4.33 & 0.99 & 1.34 \\
\hline $\begin{array}{l}\text { 159. Skill to teach and demonstrate } \\
\text { appropriate modifications in specific } \\
\text { exercises for the following groups: older } \\
\text { adults, pregnant and postnatal women, } \\
\text { obese persons, and persons with low back } \\
\text { pain }\end{array}$ & 3.80 & 3.00 & 2.86 & 2.00 & 3.33 & 2.67 & 0.94 & 0.94 \\
\hline
\end{tabular}




\begin{tabular}{|c|c|c|c|c|c|c|c|c|}
\hline \multirow[b]{3}{*}{ Exercise Science Competencies } & \multicolumn{2}{|c|}{$\begin{array}{l}\text { Exercise Science } \\
\text { Specialists }\end{array}$} & \multicolumn{2}{|c|}{$\begin{array}{l}\text { Physical education } \\
\text { teacher educators }\end{array}$} & \multicolumn{2}{|c|}{$\begin{array}{l}\text { Physical education } \\
\text { teachers }\end{array}$} & \multicolumn{2}{|c|}{$\begin{array}{l}\text { Total Delphi panel } \\
\text { responses }\end{array}$} \\
\hline & Importance & Relevance & Importance & Relevance & Importance & Relevance & Importance & Relevance \\
\hline & $M$ & $M$ & $M$ & $M$ & $M$ & $M$ & $S D$ & $S D$ \\
\hline $\begin{array}{l}\text { 166. Knowledge to describe various ways a } \\
\text { leader can take a position relative to the } \\
\text { group to enhance visibility, participant } \\
\text { interactions, and communication }\end{array}$ & 3.80 & 3.60 & 4.43 & 4.14 & 5.00 & 4.67 & 0.77 & 1.05 \\
\hline $\begin{array}{l}\text { 170. Knowledge of the properties of water } \\
\text { that affect the design of a water exercise } \\
\text { session }\end{array}$ & 3.50 & 3.22 & 3.57 & 2.71 & 3.00 & 2.33 & 1.10 & 0.99 \\
\hline $\begin{array}{l}\text { 171. Knowledge of basic music } \\
\text { fundamentals, including downbeat, } 8 \text { count, } \\
\text { and } 32 \text { count }\end{array}$ & 2.80 & 2.56 & 3.57 & 3.14 & 3.33 & 3.33 & 0.93 & 0.88 \\
\hline $\begin{array}{l}\text { 172. Skill to effectively use verbal and } \\
\text { nonverbal cues in the group exercise setting, } \\
\text { including anticipatory, motivational, safety, } \\
\text { and educational }\end{array}$ & 3.70 & 3.50 & 4.43 & 4.14 & 4.67 & 4.67 & 0.79 & 1.12 \\
\hline $\begin{array}{l}\text { 176. Knowledge, skills, and abilities to } \\
\text { prescribe and administer exercise programs } \\
\text { for apparently healthy individuals, } \\
\text { individuals at higher risk, and individuals } \\
\text { with known disease }\end{array}$ & 3.50 & 3.30 & 3.57 & 3.14 & 4.00 & 3.67 & 0.82 & 0.86 \\
\hline $\begin{array}{l}\text { 177. Ability to design, implement, and } \\
\text { evaluate individualized and group exercise } \\
\text { programs based on health history and } \\
\text { physical fitness assessments }\end{array}$ & 4.00 & 3.50 & 4.14 & 3.29 & 3.67 & 2.67 & 0.79 & 0.86 \\
\hline $\begin{array}{l}\text { 179. Knowledge, skills, and abilities to } \\
\text { calculate energy cost, VO2, METs, and } \\
\text { target heart rates and apply the information } \\
\text { to an exercise prescription }\end{array}$ & 4.10 & 3.40 & 4.00 & 3.00 & 4.00 & 3.00 & 0.83 & 1.01 \\
\hline
\end{tabular}




\begin{tabular}{|c|c|c|c|c|c|c|c|c|}
\hline \multirow[b]{3}{*}{ Exercise Science Competencies } & \multicolumn{2}{|c|}{$\begin{array}{c}\text { Exercise Science } \\
\text { Specialists }\end{array}$} & \multicolumn{2}{|c|}{$\begin{array}{l}\text { Physical education } \\
\text { teacher educators }\end{array}$} & \multicolumn{2}{|c|}{$\begin{array}{l}\text { Physical education } \\
\text { teachers }\end{array}$} & \multicolumn{2}{|c|}{$\begin{array}{l}\text { Total Delphi panel } \\
\text { responses }\end{array}$} \\
\hline & Importance & Relevance & Importance & Relevance & Importance & Relevance & Importance & Relevance \\
\hline & $M$ & $M$ & $M$ & $M$ & $M$ & $M$ & $S D$ & $S D$ \\
\hline $\begin{array}{l}\text { 180. Ability to convert weights from pounds } \\
\text { (lb) to kilograms }(\mathrm{kg}) \text { and speed from miles } \\
\text { per hour }(\mathrm{MPH}) \text { to meters per minute } \\
(\mathrm{m} \cdot \mathrm{min}-1)\end{array}$ & 3.90 & 3.10 & 3.00 & 2.71 & 3.33 & 3.33 & 1.05 & 1.08 \\
\hline $\begin{array}{l}\text { 181. Ability to convert METs to VO2 } \\
\text { expressed as } \mathrm{mL} \cdot \mathrm{kg}-1 \cdot \mathrm{min}-1, \mathrm{~L} \cdot \mathrm{min}-1 \text {, } \\
\text { and/or } \mathrm{mL} \cdot \mathrm{kg} \mathrm{FFW}-1 \cdot \mathrm{min}-1\end{array}$ & 3.60 & 2.50 & 2.71 & 1.71 & 2.67 & 1.67 & 1.09 & 1.02 \\
\hline $\begin{array}{l}\text { 182. Ability to calculate the energy cost in } \\
\text { METs and kilocalories for given exercise } \\
\text { intensities in stepping exercise, cycle } \\
\text { ergometry, and during horizontal and } \\
\text { graded walking and running }\end{array}$ & 3.50 & 2.70 & 3.43 & 2.43 & 3.33 & 2.67 & 0.89 & 1.05 \\
\hline $\begin{array}{l}\text { 183. Knowledge of the approximate METs } \\
\text { for various sport, recreational, and work } \\
\text { tasks }\end{array}$ & 4.10 & 3.40 & 3.43 & 2.71 & 3.33 & 2.67 & 0.85 & 0.94 \\
\hline $\begin{array}{l}\text { 184. Ability to prescribe exercise intensity } \\
\text { based on VO2 data for different modes of } \\
\text { exercise, including graded and horizontal } \\
\text { running and walking, cycling, and stepping }\end{array}$ & 3.70 & 2.80 & 3.71 & 2.43 & 3.00 & 2.33 & 0.99 & 0.94 \\
\hline $\begin{array}{l}\text { 185. Ability to explain and implement } \\
\text { exercise prescription guidelines for } \\
\text { apparently health clients, increased risk } \\
\text { clients, and clients with controlled disease }\end{array}$ & 3.60 & 3.50 & 3.49 & 2.43 & 3.00 & 2.33 & 0.94 & 1.23 \\
\hline $\begin{array}{l}\text { 186. Ability to adapt frequency, intensity, } \\
\text { duration, mode, progression, level of } \\
\text { supervision, and monitoring techniques in } \\
\text { exercise programs for patients with } \\
\text { controlled chronic disease, musculoskeletal } \\
\text { problems, pregnancy and/or postpartum and } \\
\text { exercise-induced asthma }\end{array}$ & 3.40 & 3.10 & 2.57 & 2.00 & 2.33 & 2.33 & 0.83 & 0.99 \\
\hline
\end{tabular}




\begin{tabular}{|c|c|c|c|c|c|c|c|c|}
\hline \multirow{3}{*}{ Exercise Science Competencies } & \multicolumn{2}{|c|}{$\begin{array}{l}\text { Exercise Science } \\
\text { Specialists }\end{array}$} & \multicolumn{2}{|c|}{$\begin{array}{l}\text { Physical education } \\
\text { teacher educators }\end{array}$} & \multicolumn{2}{|c|}{$\begin{array}{l}\text { Physical education } \\
\text { teachers }\end{array}$} & \multicolumn{2}{|c|}{$\begin{array}{l}\text { Total Delphi panel } \\
\text { responses }\end{array}$} \\
\hline & Importance & Relevance & Importance & Relevance & Importance & Relevance & Importance & Relevance \\
\hline & $M$ & $M$ & $M$ & $M$ & $M$ & $M$ & $S D$ & $S D$ \\
\hline $\begin{array}{l}\text { 188. Skill in the use of various methods for } \\
\text { establishing and monitoring levels of } \\
\text { exercise intensity, including heart rate, } \\
\text { RPE, and METs }\end{array}$ & 4.20 & 3.80 & 4.29 & 3.86 & 3.67 & 3.33 & 0.81 & 0.85 \\
\hline $\begin{array}{l}\text { 189. Knowledge of special precautions and } \\
\text { modifications of exercise programming for } \\
\text { participation at altitude, different ambient } \\
\text { temperatures, humidity, and pollution }\end{array}$ & 4.30 & 4.00 & 4.29 & 3.43 & 4.00 & 4.00 & 0.44 & 0.77 \\
\hline $\begin{array}{l}\text { 193. Knowledge of the advantages and } \\
\text { disadvantages of implementation of } \\
\text { interval, continuous, and circuit training } \\
\text { programs }\end{array}$ & 4.20 & 3.50 & 4.50 & 4.33 & 4.67 & 4.33 & 0.76 & 0.94 \\
\hline $\begin{array}{l}\text { 195. Ability to discuss the advantages and } \\
\text { disadvantages of various commercial } \\
\text { exercise equipment in developing } \\
\text { cardiorespiratory fitness, muscular } \\
\text { strength/endurance }\end{array}$ & 3.80 & 3.50 & 3.83 & 3.33 & 4.00 & 3.33 & 0.83 & 0.84 \\
\hline $\begin{array}{l}\text { 196. Knowledge of the types of exercise } \\
\text { programs available in the community and } \\
\text { how these programs are appropriate for } \\
\text { various populations }\end{array}$ & 3.90 & 3.90 & 3.83 & 3.00 & 4.67 & 4.33 & 0.82 & 1.11 \\
\hline $\begin{array}{l}\text { 198. Knowledge of the concept of } \\
\text { periodization as it relates to strength and } \\
\text { endurance exercise programming }\end{array}$ & 3.90 & 3.50 & 4.00 & 3.20 & 4.00 & 3.33 & 0.87 & 0.92 \\
\hline $\begin{array}{l}\text { 205. Ability to differentiate between fat } \\
\text { soluble and water-soluble vitamins }\end{array}$ & 3.20 & 2.60 & 3.50 & 2.50 & 3.67 & 3.00 & 1.07 & 0.83 \\
\hline $\begin{array}{l}\text { 208. Knowledge of the importance of } \\
\text { calcium and iron in women's health }\end{array}$ & 3.90 & 3.90 & 4.00 & 3.67 & 4.33 & 4.00 & 0.67 & 0.69 \\
\hline 210. Knowledge of the number of & 4.20 & 3.00 & 3.87 & 3.33 & 3.67 & 3.67 & 0.91 & 1.03 \\
\hline
\end{tabular}

kilocalories in one gram of carbohydrate,

fat, protein, and alcohol 


\begin{tabular}{|c|c|c|c|c|c|c|c|c|}
\hline \multirow[b]{3}{*}{ Exercise Science Competencies } & \multicolumn{2}{|c|}{$\begin{array}{l}\text { Exercise Science } \\
\text { Specialists }\end{array}$} & \multicolumn{2}{|c|}{$\begin{array}{l}\text { Physical education } \\
\text { teacher educators }\end{array}$} & \multicolumn{2}{|c|}{$\begin{array}{l}\text { Physical education } \\
\text { teachers }\end{array}$} & \multicolumn{2}{|c|}{$\begin{array}{l}\text { Total Delphi panel } \\
\text { responses }\end{array}$} \\
\hline & Importance & Relevance & Importance & Relevance & Importance & Relevance & Importance & Relevance \\
\hline & $M$ & $M$ & $M$ & $M$ & $M$ & $M$ & $S D$ & $S D$ \\
\hline $\begin{array}{l}\text { 211. Knowledge of the number of kcals } \\
\text { equivalent to losing } 1 \mathrm{lb} \text { of body fat }\end{array}$ & 4.10 & 3.60 & 3.83 & 3.33 & 3.67 & 3.67 & 0.97 & 1.02 \\
\hline $\begin{array}{l}\text { 213. Ability to describe the health } \\
\text { implications of variation in body fat } \\
\text { distribution patterns and the significance of } \\
\text { waist to hip ratio }\end{array}$ & 4.10 & 3.90 & 4.33 & 3.83 & 3.33 & 3.00 & 0.78 & 0.81 \\
\hline $\begin{array}{l}\text { 215. Knowledge of common nutritional } \\
\text { ergogenic aids, the purported mechanism of } \\
\text { action, and any risk and/or benefit (e.g., } \\
\text { carbohydrates, protein/amino acids, } \\
\text { vitamins) }\end{array}$ & 4.00 & 3.70 & 3.83 & 3.67 & 3.33 & 2.67 & 0.76 & 0.84 \\
\hline $\begin{array}{l}\text { 216. Knowledge of nutritional factors } \\
\text { related to the female athlete triad syndrome } \\
\text { (i.e., eating disorders, menstrual cycle } \\
\text { abnormalities, and osteoporosis) }\end{array}$ & 3.80 & 3.56 & 4.00 & 3.43 & 3.67 & 3.00 & 1.04 & 1.02 \\
\hline $\begin{array}{l}\text { 217. Knowledge of the NIH Consensus } \\
\text { statement regarding health risks of obesity, } \\
\text { Nutrition for Physical Fitness Position } \\
\text { Paper of the American Dietetic Association, } \\
\text { and the ACSM Position Stand on proper } \\
\text { and improper weight loss programs }\end{array}$ & 4.40 & 3.70 & 4.29 & 3.57 & 4.00 & 3.33 & 0.57 & 0.99 \\
\hline $\begin{array}{l}\text { 218. Knowledge of NCEP II guidelines for } \\
\text { lipid management }\end{array}$ & 3.80 & 3.20 & 2.83 & 2.50 & 3.00 & 2.67 & 0.96 & 0.81 \\
\hline $\begin{array}{l}\text { 220. Ability to describe and use the } \\
\text { documentation required when a client } \\
\text { shows signs or symptoms during an } \\
\text { exercise session and should be referred to a } \\
\text { physician }\end{array}$ & 3.60 & 3.44 & 4.00 & 2.86 & 4.33 & 4.00 & 0.76 & 0.95 \\
\hline $\begin{array}{l}\text { 221. Ability to create and maintain records } \\
\text { pertaining to participant exercise adherence, } \\
\text { retention, and goal setting }\end{array}$ & 3.90 & 3.70 & 4.17 & 4.00 & 4.33 & 4.00 & 0.71 & 0.88 \\
\hline
\end{tabular}




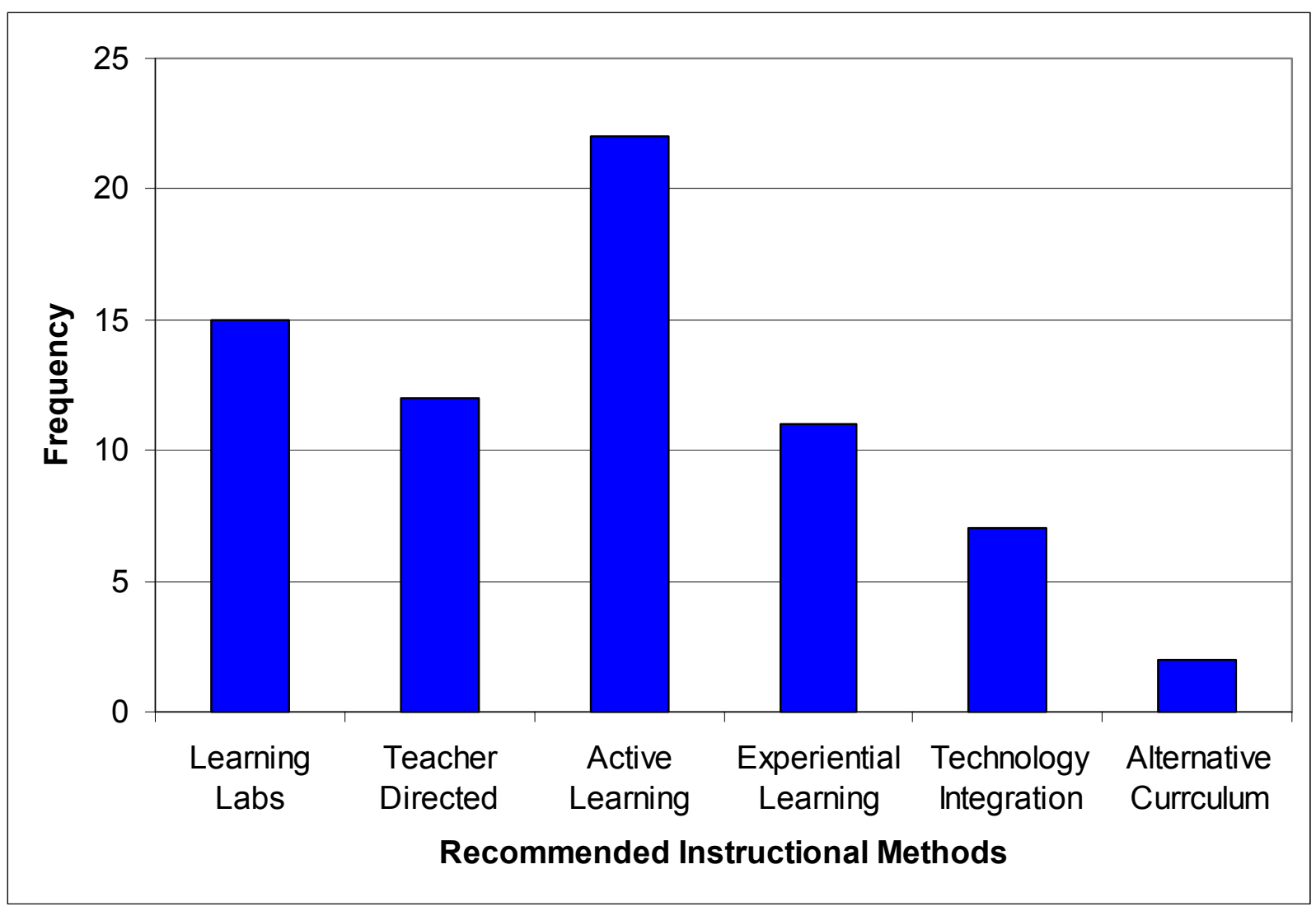

Figure 1 - Recommended instructional methods for delivering exercise science content in PETE 


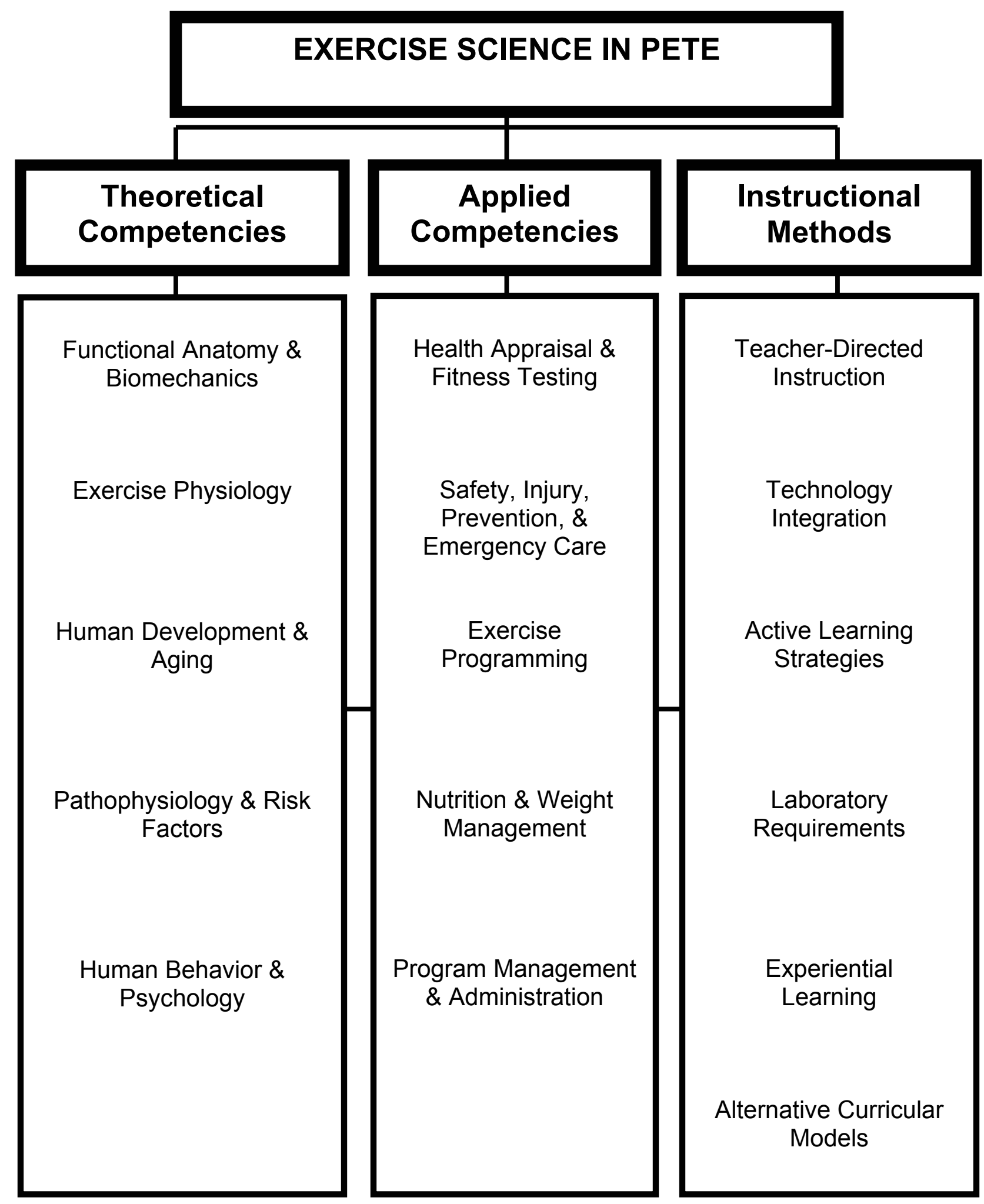

Figure 2 - General summary of Delphi panel findings regarding exercise science content and recommended instructional methods in PETE 
COMPONENTS OF THE PETE CURRICULUM
SAMPLE COURSE WORK IN PETE

\section{Practical Field Experience}

- To provide a series of progressive practice teaching opportunities

- Sample field experiences:

Instructional Modeling \& Observation

Teaching Simulations

Peer Teaching

Teaching Labs

Clinical Field Placements

Student Teaching Placements at the Elementary, Middle, \& Secondary School Levels

\section{Foundational} Subdisciplinary

- To provide a conceptual framework for appropriate professional practice

- To initiate the key strands or themes that extend throughout the rest of the PETE curriculum
Introduction to PE

Anatomy \& Physiology

Behavioral Principles

Exercise Physiology

Biomechanics

Motor Development

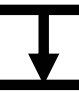

Curriculum \& Instruction

- To develop pedagogical knowledge \& skills required for effective instructional design, implementation, \& assessment in physical education

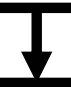

Instructional Systems in

Physical Education

Measurement \&

Evaluation

Physical Education Curriculum

Qualitative Movement Analysis

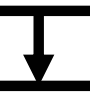

Teaching of Elementary School-Aged Children

Teaching of Middle

- To integrate subject knowledge \& pedagogical knowledge in specific instructional situations
School-Aged Children

Teaching of Secondary School-Aged Children

Figure 3 - Overview of a conceptual framework for the PETE curriculum 


\section{Anatomy \& Biomechanics}

- Functional anatomy and biomechanics as related to physical activity, exercise, and sport performance

- Basic anatomy of the cardiovascular system

\section{Exercise Physiology}

- Basic exercise physiology related to cardiovascular and musculoskeletal systems at rest and during exercise

- Carbohydrates, fats, proteins and the contribution of the body's energy systems during various forms of physical activity

- Components of health-related physical fitness: cardiovascular endurance, muscular strength, muscular endurance, flexibility, and body composition

- Acute and chronic physiological responses to cardiovascular training

- Acute and chronic physiological responses to resistance training

- Physiological basis for muscular fatigue as related to task intensity and duration

- Basic principles of physical activity program design (specificity, progression, overload, reversibility)

- Physiological principles related to warm-up and cool-down

\section{Pathophysiology \& Risk Factors}

- Risk factor concept of coronary artery disease and the relationship between genetics and lifestyle

- Determination of those risk factors that may be modifiable through physical activity

- Influence of lifestyle factors (nutrition and physical activity) and heredity on blood lipid and lipoprotein profiles

\section{Human Development \& Aging}

- Health-related benefits of physical activity for people of all ages

- Developmental changes that occur from childhood to old age

- Special benefits and risks associated with physical activity in children and youth (e.g., children and adolescent strength training)

- Unique physiological adaptations to various forms of physical activity in children and adolescents

- Influence of weight-bearing activity on optimal bone growth and development during childhood and adolescence

- Modifications in physical activity program design to account for developmental and individual differences

\section{Human Behavior \& Psychology}

- Psychological benefits of regular participation in physical activity

- Environmental determinants of physical activity and exercise participation

- Promotion of positive attitudes and perceptions regarding physical activity and exercise

- Strategies for facilitating behavior change including goal setting, behavioral contracts, reinforcement, and social support

- Techniques for enhancing participant intrinsic and extrinsic motivation (e.g., posters, recognition, bulletin boards, games, competitions)

- Use of various educational theories, instructional methods, and behavioral management principles to establish a safe and supportive physical activity environment

- Signs and symptoms of disordered eating and the appropriate procedures for referral

Figure 4 - Summary of theoretical exercise science competencies in PETE 


\section{Health Appraisal \& Fitness Testing}

- Role of fitness testing in achieving national and state standards for physical education

- Appropriateness of norm- versus criterionreferenced physical fitness tests

- Selection of appropriate test protocol for assessing cardiovascular endurance, body composition, muscular fitness, and flexibility

- Modification of testing protocols and procedures for children and adolescents

- Steps needed to prepare children for physical fitness testing including teaching test purpose, protocol, equipment use, and scoring

- Monitoring exercise intensity through the use of heart rate (manually and with heart rate monitors) and rating of perceived exertion

- Use of pedometers for measuring physical activity participation

\section{Safety, Injury Prevention, Emergency Care}

- Legal implications of risk management and emergency preparation in physical education

- Factors that contribute to the maintenance of a safe exercise environment

- Standard first aid procedures and basic life support/CPR certification

- Emergency procedures in an exercise setting (including First Aid and CPR certification)

- Problems associated with contraindicated exercises and the potential safety concerns

\section{Program Management \& Administration}

- Administration and delivery of a complete health fitness program

- Promotion of physical activity through the development of educational programs and materials

\section{Exercise Programming}

- Amounts of physical activity for improved health and physical fitness (guidelines for children, adolescents, and adults)

- Training variables of frequency, intensity, time, type and physical activity program design

- Sequencing of components within an exercise session (e.g., warm-up, aerobic training, cooldown, resistance training, flexibility)

- Teaching activities for warm-up/cool-down

- Teaching various exercise modes (e.g., steps, cycles, groups) and formats (e.g., continuous, interval, circuit) for cardiovascular endurance

- Teaching progression of resistance training exercises for major muscle groups (e.g., stability balls, weights, partner-resisted)

- Teaching flexibility exercises for all major muscle and tendon groups

- Effective communication and modification of exercises to account for different fitness levels

- Monitoring exercise intensity using target heart rate and rating of perceived exertion

- Measuring program effectiveness through use of physical activity logs and periodic retesting

\section{Nutrition \& Weight Management}

- Influence of body composition on health

- USRDA Food Pyramid and the role of daily caloric intake in healthy weight management

- Importance of maintaining adequate hydration before, during, and after exercise

- Strategies for teaching nutrition and the role of diet and exercise in weight management

- Effectiveness of various diet and exercise combinations for modifying body composition

- Improper weight loss practices and concerns

Figure 5 - Summary of applied exercise science competencies in PETE 


\section{APPENDIX A}

Modified Delphi Investigation of Exercise Science in Physical Education Teacher Education Pilot Study 


\section{PHONE SCRIPT FOR PILOT TEST PARTICIPANT RECRUITMENT}

"Hello, my name is Sean Bulger and I am calling to request your participation as an expert panel member for the pilot test of my dissertation research titled a Modified Delphi Investigation of Exercise Science in Physical Education Teacher Education. As explained in my previous e-mail, this research project will involve the administration of a modified Delphi Procedure to determine the critical exercise science competencies that should be included in the physical education teacher education undergraduate curriculum. Your specific contribution to this research project will be to critique the survey items that will eventually be employed in terms of completeness and content validity. Are you interested in participating as an expert panel member for this pilot test?"

\section{YES/NO (IF "NO” GO TO 1 / IF "YES" GO TO 2)}

1. "Thank you for your time and consideration."

2. "Thank you for agreeing to participate, your time and cooperation are greatly appreciated.

Within the next week, I will mail you an information packet that includes some additional details regarding your participation and a copy of the survey instrument. How would you like me to address this mailing?” (RECORD MAILING ADDRESS IN THE SPACE PROVIDED BELOW) "Thanks again for agreeing to participate. Your expert contribution is critical to the success of this research project." 


\section{(ON LETTERHEAD STATIONARY)}

(PANEL MEMBER NAME)

(MAILING ADDRESS)

(CITY, STATE, ZIP CODE)

(DATE)

Dear

Thank you for agreeing to serve as an expert judge for the instrument design phase of my dissertation, titled a Modified Delphi Investigation of Exercise Science in Physical Education Teacher Education. As described in our previous phone conversation, you were chosen because of your considerable expertise in the area of exercise science and/or its eventual practical application in school-based physical education programs.

The ultimate purpose of this research project is to determine a list of critical exercise science competencies that should be included in the physical education teacher education curriculum. The Delphi panel members will be asked to rate a series of exercise science competencies in terms of their importance and pedagogical relevance for prospective K-12 physical education teachers.

Your specific role in this project will be to critique the proposed survey items in terms of their completeness and content validity. The items that you are evaluating were adapted directly from the American College of Sports Medicine $(2000)^{1}$ and reproduced with the permission of the copyright owner, Lippincott Williams \& Wilkins. Please read the instructions that have been provided prior to completing the survey. If you have any questions regarding your task, contact me at (715) 836-3722 or bulgersm@uwec.edu.

The results of this research project will be used to fulfill the requirements for my doctoral dissertation, which I am completing at West Virginia University. Your participation in this preliminary phase of the research process is entirely voluntary and you do not need to respond to every item on the questionnaire. You may be assured of complete confidentiality regarding all of your responses and comments. I will send you the final results of the study upon its completion.

If possible, please return the completed survey to me within one week of receiving it. Additionally, send a current copy of your curriculum vitae or resume if you would like it included in my dissertation as recognition of your contribution to the project. Use the return envelope that I have provided for your convenience and accept the enclosed gift as a small gesture of my appreciation for sharing your time, effort, and expertise. Thanks again for your assistance and cooperation.

Sincerely,

Sean M. Bulger

Assistant Professor

${ }^{1}$ ACSM. (2000). ACSM's Guidelines for Exercise Testing and Prescription (6 $6^{\text {th }}$ edition). Baltimore, MD: Lippincott Williams \& Wilkins. 


\title{
Modified Delphi Investigation of Exercise Science in Physical Education Teacher Education
}

\author{
Survey Instrument Design
}

March 2003

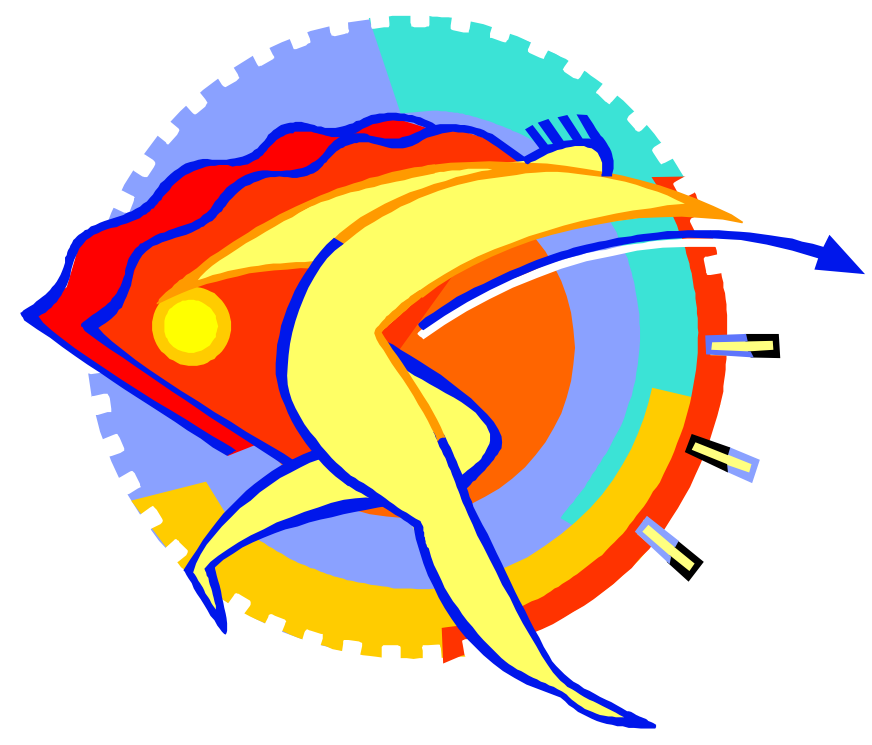

Sean M. Bulger, Assistant Professor

University of Wisconsin-Eau Claire

Department of Kinesiology \& Athletics

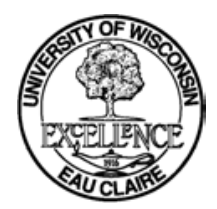




\section{Introduction}

The survey items you have agreed to review will be used during a subsequent research project to generate a consensus of expert opinion regarding the critical theoretical and applied exercise science competencies that prospective physical educators need to learn within the physical education teacher education curriculum. The Delphi Method will be employed to address this primary research question. The Delphi Method is a research protocol that involves (a) the identification and selection of a small panel of experts on a particular topic, (b) the use of multiple rounds of surveying as a means to collect expert opinion, and (c) the attainment of group consensus through the provision of regular feedback.

Your expertise and judgment remains central to the success of this project because you are helping to identify the survey items that will eventually be considered by the actual Delphi panel members. Due to the nature of both the involved research question and the Delphi Method, the list of potential survey items I am asking you to evaluate is rather extensive. The items that you are evaluating were adapted directly from the American College of Sports Medicine (2000) ${ }^{1}$ and reproduced with the permission of the copyright owner, Lippincott Williams \& Wilkins.

Please know that your commitment to the timely completion of this project is greatly appreciated.

\section{Table of Contents}

Instructions.................................................................................... ii

Section 1: Anatomy \& Biomechanics (Items 1-20) ..................................... 1

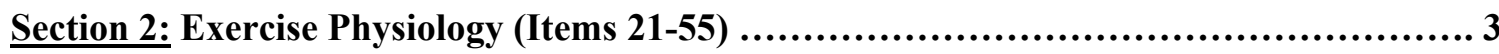

Section 3: Human Development \& Aging (Items 56-65) ................................ 6

Section 4: Pathophysiology/Risk Factors (Items 66-77) ............................... 7

Section 5: Human Behavior \& Psychology (Items 78-85) .............................. 8

Section 6: Health Appraisal \& Fitness Testing (Items 86-114) .............................. 9

Section 7: Safety, Injury Prevention, and Emergency Care (Items 115-130) ................. 11

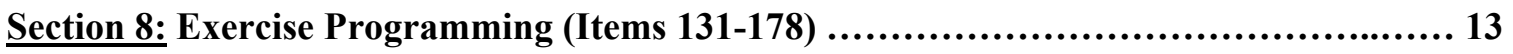

Section 9: Nutrition \& Weight Management (Items 179-196) ........................... 17

Section 10: Program \& Administration/Management (Items 197-206) ................... 18

${ }^{1}$ ACSM. (2000). ACSM's Guidelines for Exercise Testing and Prescription (6 ${ }^{\text {th }}$ edition). Baltimore, MD: Lippincott Williams \& Wilkins. 


\section{To: $\quad$ Expert Judges}

From: Sean M. Bulger, Assistant Professor

RE: Instructions for Judging the Survey Instrument

Again, thank you very much for your assistance in completing the instrument design phase of this research project. The survey instrument you are helping to design will ultimately include a comprehensive list of critical theoretical and applied exercise science competencies that are recommended for inclusion in the physical education teacher education curriculum. The goal of this preliminary phase of the research project is to produce a valid list of theoretical and applied competencies that comprehensively represents the available body of knowledge in the area of exercise science.

\section{YOUR FIRST TASK IS TO DETERMINE CONTENT VALIDITY}

For the purpose of this study, content validity will be used to determine whether or not the item should be included on the final questionnaire. In other words, is the item a valid theoretical or applied exercise science competency that could potentially be incorporated in the physical education teacher education curriculum? Remember that you are not judging the item's importance or relevance, only its validity.

Please rate each questionnaire item individually: A rating of (5) or (4) means that the item should be retained; a rating of (3) means that you are neutral or undecided; and a rating of (2) or (1) means that item should be dropped from the study because it is not a valid theoretical or applied exercise science competency.

\section{YOUR SECOND TASK IS TO DETERMINE COMPLETENESS}

Please feel free to add any items that you feel are needed to provide a higher degree of content validity. Remember that your goal is to produce a comprehensive list of theoretical and applied exercise science competencies that could potentially be included in the physical education teacher education curriculum. Please write any competencies that you would like to see added to each section in the space provided.

After you finish assessing and modifying the instrument, please respond to the following questions concerning the overall quality of the instrument by circling either YES or NO.

In your expert opinion, does the instrument (as amended) have content validity? YES NO

In your expert opinion, does the instrument (as amended) represent a comprehensive overview of the theoretical and applied competencies that could be potentially included in the physical education teacher education undergraduate program? YES NO

Thanks again and please feel free to contact me directly with any questions at (715) 836-3722 or bulgersm@uwec.edu

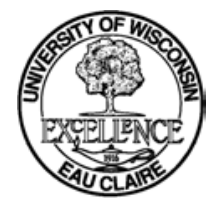




\section{Modified Delphi Investigation of Exercise Science in Physical Education Teacher Education Survey Instrument Design}

Instructions: Rate the content validity of each exercise science competency using the scale that has been provided and improve the completeness of the instrument by recommending any modifications and/or additional competencies in the available space.

\begin{tabular}{|c|c|c|c|c|c|}
\hline \multirow[t]{3}{*}{ Category \& Item Name } & \multicolumn{5}{|c|}{ Content Validity } \\
\hline & \multicolumn{2}{|c|}{ Drop Item } & \multirow[b]{2}{*}{3} & \multicolumn{2}{|c|}{ Keep Item } \\
\hline & 1 & 2 & & 4 & 5 \\
\hline \multicolumn{6}{|l|}{ Section 1 Anatomy \& Biomechanics } \\
\hline 1. Knowledge of anatomy as it relates to exercise and health & 1 & 2 & 3 & 4 & 5 \\
\hline 2. Knowledge of the basic structures of bone, skeletal muscle, and connective tissues & 1 & 2 & 3 & 4 & 5 \\
\hline 3. Knowledge of the basic anatomy of the cardiovascular system and respiratory system & 1 & 2 & 3 & 4 & 5 \\
\hline $\begin{array}{l}\text { 4. Ability to identify the major bones and muscles: Major muscles include, but are not limited to: trapezius, } \\
\text { pectoralis major, latissimus dorsi, biceps, triceps, rectus abdominus, internal and external obliques, erector } \\
\text { spinae, gluteus maximus, quadriceps, hamstrings, adductors, abductors, and gastrocnemius }\end{array}$ & 1 & 2 & 3 & 4 & 5 \\
\hline $\begin{array}{l}\text { 5. Knowledge of the definition of the following terms: supination, pronation, flexion, extension, adduction, } \\
\text { abduction, hyperextension, rotation, circumduction, agonist, antagonist, and stabilizer }\end{array}$ & 1 & 2 & 3 & 4 & 5 \\
\hline 6. Ability to identify the joints of the body & 1 & 2 & 3 & 4 & 5 \\
\hline 7. Knowledge of the biomechanical aspects of exercise participation & 1 & 2 & 3 & 4 & 5 \\
\hline 8. Knowledge to identify the plane in which each muscle action occurs & 1 & 2 & 3 & 4 & 5 \\
\hline $\begin{array}{l}\text { 9. Knowledge of the interrelationships among center of gravity, base of support, balance, stability, and proper } \\
\text { spinal alignment }\end{array}$ & 1 & 2 & 3 & 4 & 5 \\
\hline 10. Ability to describe the following curvatures of the spine: lordosis, scoliosis, and kyphosis & 1 & 2 & 3 & 4 & 5 \\
\hline $\begin{array}{l}\text { 11. Knowledge of and skill to demonstrate exercises designed to enhance muscular strength and/or endurance of } \\
\text { specific major muscle groups }\end{array}$ & 1 & 2 & 3 & 4 & 5 \\
\hline 12. Knowledge of and skill to demonstrate exercise for enhancing musculoskeletal flexibility & 1 & 2 & 3 & 4 & 5 \\
\hline 13. Knowledge to describe the myotatic stretch reflex & 1 & 2 & 3 & 4 & 5 \\
\hline 14. Knowledge to identify the primary action and joint range of motion for each major muscle group & 1 & 2 & 3 & 4 & 5 \\
\hline 15. Knowledge of functional anatomy and biomechanics & 1 & 2 & 3 & 4 & 5 \\
\hline 16. Knowledge of the structure and ability to describe movements for the major joints of the body & 1 & 2 & 3 & 4 & 5 \\
\hline
\end{tabular}


Appendix A 120

17. Ability to locate the anatomic landmarks for palpation of peripheral pulses 34

18. Ability to locate the brachial artery and correctly place the cuff and stethoscope in position for blood pressure 1 measurement

19. Ability to locate common sites for measurement of skinfold thickness and circumferences (for determination of body composition and waist-hip ratio)

20. Knowledge of the biomechanical principles that underlie performance of the following activities: walking, jogging, running, swimming, cycling, weight lifting, and carrying or moving objects

\section{Completeness Please list any additional anatomy/biomechanics competencies you would recommend in the space provided:}

\begin{tabular}{|l|}
\hline \\
\hline \\
\hline \\
\hline \\
\hline \\
\hline \\
\hline \\
\hline
\end{tabular}




\begin{tabular}{|c|c|c|c|c|c|}
\hline \multirow[t]{3}{*}{ Category \& Item Name } & \multicolumn{5}{|c|}{ Content Validity } \\
\hline & \multicolumn{2}{|c|}{ Drop Item } & \multirow[b]{2}{*}{3} & \multicolumn{2}{|c|}{ Keep Item } \\
\hline & 1 & 2 & & 4 & 5 \\
\hline \multicolumn{6}{|l|}{ Section 2 Exercise Physiology } \\
\hline 21. Basic knowledge of exercise physiology as it relates to exercise prescription & 1 & 2 & 3 & 4 & 5 \\
\hline 22. Ability to define aerobic and anaerobic metabolism & 1 & 2 & 3 & 4 & 5 \\
\hline 23. Knowledge of the role of aerobic and anaerobic energy systems in the performance of various activities & 1 & 2 & 3 & 4 & 5 \\
\hline $\begin{array}{l}\text { 24. Knowledge of the following terms: ischemia, angina pectoris, tachycardia, bradycardia, arrhythmia, } \\
\text { myocardial infarction, cardiac output, stroke volume, lactic acid, oxygen consumption, hyperventilation, } \\
\text { systolic blood pressure, diastolic blood pressure, and anaerobic threshold }\end{array}$ & 1 & 2 & 3 & 4 & 5 \\
\hline 25. Knowledge of the role of carbohydrates, fats, and proteins as fuels for aerobic and anaerobic metabolism & 1 & 2 & 3 & 4 & 5 \\
\hline $\begin{array}{l}\text { 26. Knowledge of the components of fitness: cardiorespiratory fitness, muscular strength, muscular endurance, } \\
\text { flexibility, and body composition }\end{array}$ & 1 & 2 & 3 & 4 & 5 \\
\hline $\begin{array}{l}\text { 27. Knowledge to describe normal cardiorespiratory responses to static and dynamic exercise in terms of heart } \\
\text { rate, blood pressure, and oxygen consumption }\end{array}$ & 1 & 2 & 3 & 4 & 5 \\
\hline $\begin{array}{l}\text { 28. Knowledge of how heart rate, blood pressure, and oxygen consumption responses change with adaptation to } \\
\text { chronic exercise training }\end{array}$ & 1 & 2 & 3 & 4 & 5 \\
\hline 29. Knowledge of the physiological adaptations associated with strength training & 1 & 2 & 3 & 4 & 5 \\
\hline $\begin{array}{l}\text { 30. Ability to identify and apply to both groups and individuals methods used to monitor exercise intensity, } \\
\text { including heart rate and rating of perceived exertion }\end{array}$ & 1 & 2 & 3 & 4 & 5 \\
\hline 31. Knowledge of the physiological principles related to warm-up and cool-down & 1 & 2 & 3 & 4 & 5 \\
\hline 32. Knowledge of the common theories of muscle fatigue and delayed onset muscle soreness (DOMS) & 1 & 2 & 3 & 4 & 5 \\
\hline $\begin{array}{l}\text { 33. Knowledge of exercise physiology including the role of aerobic and anaerobic metabolism, muscle } \\
\text { physiology, cardiovascular physiology, and respiratory physiology at rest and during exercise. In addition, } \\
\text { demonstrate an understanding of the components of physical fitness, the effects of aerobic and strength and/or } \\
\text { resistance training on the fitness components and the effects of chronic disease }\end{array}$ & 1 & 2 & 3 & 4 & 5 \\
\hline $\begin{array}{l}\text { 34. Knowledge of the physiological adaptations that occur at rest and during submaximal and maximal exercise } \\
\text { following chronic aerobic and anaerobic exercise training }\end{array}$ & 1 & 2 & 3 & 4 & 5 \\
\hline $\begin{array}{l}\text { 35. Knowledge of the differences in cardiorespiratory response to acute graded exercise between conditioned and } \\
\text { unconditioned individuals }\end{array}$ & 1 & 2 & 3 & 4 & 5 \\
\hline 36. Knowledge of the structure of the skeletal muscle fiber and the basic mechanism of contraction & 1 & 2 & 3 & 4 & 5 \\
\hline
\end{tabular}


Appendix A 122

\begin{tabular}{|c|c|c|c|c|c|}
\hline 37. Knowledge of the characteristics of fast and slow twitch fibers & 1 & 2 & 3 & 4 & 5 \\
\hline 38. Knowledge of the sliding filament theory of muscle contraction & 1 & 2 & 3 & 4 & 5 \\
\hline 39. Knowledge of twitch, summation, and tetanus with respect to muscle contraction & 1 & 2 & 3 & 4 & 5 \\
\hline 40. Ability to discuss the physiological principles involved in promoting gains in muscular strength and endurance & 1 & 2 & 3 & 4 & 5 \\
\hline $\begin{array}{l}\text { 41. Ability to define muscular fatigue as it relates to task, intensity, duration, and the accumulative effects of } \\
\text { exercise }\end{array}$ & 1 & 2 & 3 & 4 & 5 \\
\hline $\begin{array}{l}\text { 42. Knowledge of the relationship between the number of repetitions, intensity, number of sets, and rest with } \\
\text { regard to strength training }\end{array}$ & 1 & 2 & 3 & 4 & 5 \\
\hline 43. Knowledge of the basic properties of cardiac muscle and the normal pathways of conduction in the heart & 1 & 2 & 3 & 4 & 5 \\
\hline $\begin{array}{l}\text { 44. Knowledge of the response of the following variables to acute exercise: heart rate, stroke volume, cardiac } \\
\text { output, pulmonary ventilation, tidal volume, respiratory rate, and arteriovenous oxygen difference }\end{array}$ & 1 & 2 & 3 & 4 & 5 \\
\hline $\begin{array}{l}\text { 45. Knowledge of the differences in the cardiorespiratory responses to static exercise compared with dynamic } \\
\text { exercise, including possible hazards and contraindications }\end{array}$ & 1 & 2 & 3 & 4 & 5 \\
\hline $\begin{array}{l}\text { 46. Ability to describe how each of the following differs from the normal condition: premature atrial contractions } \\
\text { and premature ventricular contractions }\end{array}$ & 1 & 2 & 3 & 4 & 5 \\
\hline 47. Knowledge of blood pressure responses associated with acute exercise, including changes in body position & 1 & 2 & 3 & 4 & 5 \\
\hline $\begin{array}{l}\text { 48. Knowledge of and ability to describe the implications of ventilatory threshold (anaerobic threshold) as it } \\
\text { relates to exercise training and cardiorespiratory assessment }\end{array}$ & 1 & 2 & 3 & 4 & 5 \\
\hline $\begin{array}{l}\text { 49. Knowledge of and ability to describe the physiological adaptations of the respiratory system that occur at rest } \\
\text { and during submaximal and maximal exercise following chronic aerobic and anaerobic training }\end{array}$ & 1 & 2 & 3 & 4 & 5 \\
\hline $\begin{array}{l}\text { 50. Ability to describe how each of the following differs from the normal condition: dyspnea, hypoxia, and } \\
\text { hypoventilation }\end{array}$ & 1 & 2 & 3 & 4 & 5 \\
\hline $\begin{array}{l}\text { 51. Knowledge of and ability to discuss the physiological basis of the major components of physical fitness: } \\
\text { flexibility, cardiovascular fitness, muscular strength, muscular endurance, and body composition }\end{array}$ & 1 & 2 & 3 & 4 & 5 \\
\hline 52. Ability to explain how the principle of specificity relates to the components of fitness & 1 & 2 & 3 & 4 & 5 \\
\hline $\begin{array}{l}\text { 53. Ability to explain the concept of detraining or reversibility of conditioning and its implications in fitness } \\
\text { programs }\end{array}$ & 1 & 2 & 3 & 4 & 5 \\
\hline $\begin{array}{l}\text { 54. Ability to discuss the physical and psychological signs of overtraining and to provide recommendations for } \\
\text { these problems }\end{array}$ & 1 & 2 & 3 & 4 & 5 \\
\hline $\begin{array}{l}\text { 55. Ability to describe the physiological and metabolic responses to exercise associated with chronic disease } \\
\text { (heart disease, hypertension, diabetes mellitus, and pulmonary disease) }\end{array}$ & 1 & 2 & 3 & 4 & 5 \\
\hline
\end{tabular}


Completeness Please list any additional exercise physiology competencies you would recommend in the space provided:

(2)




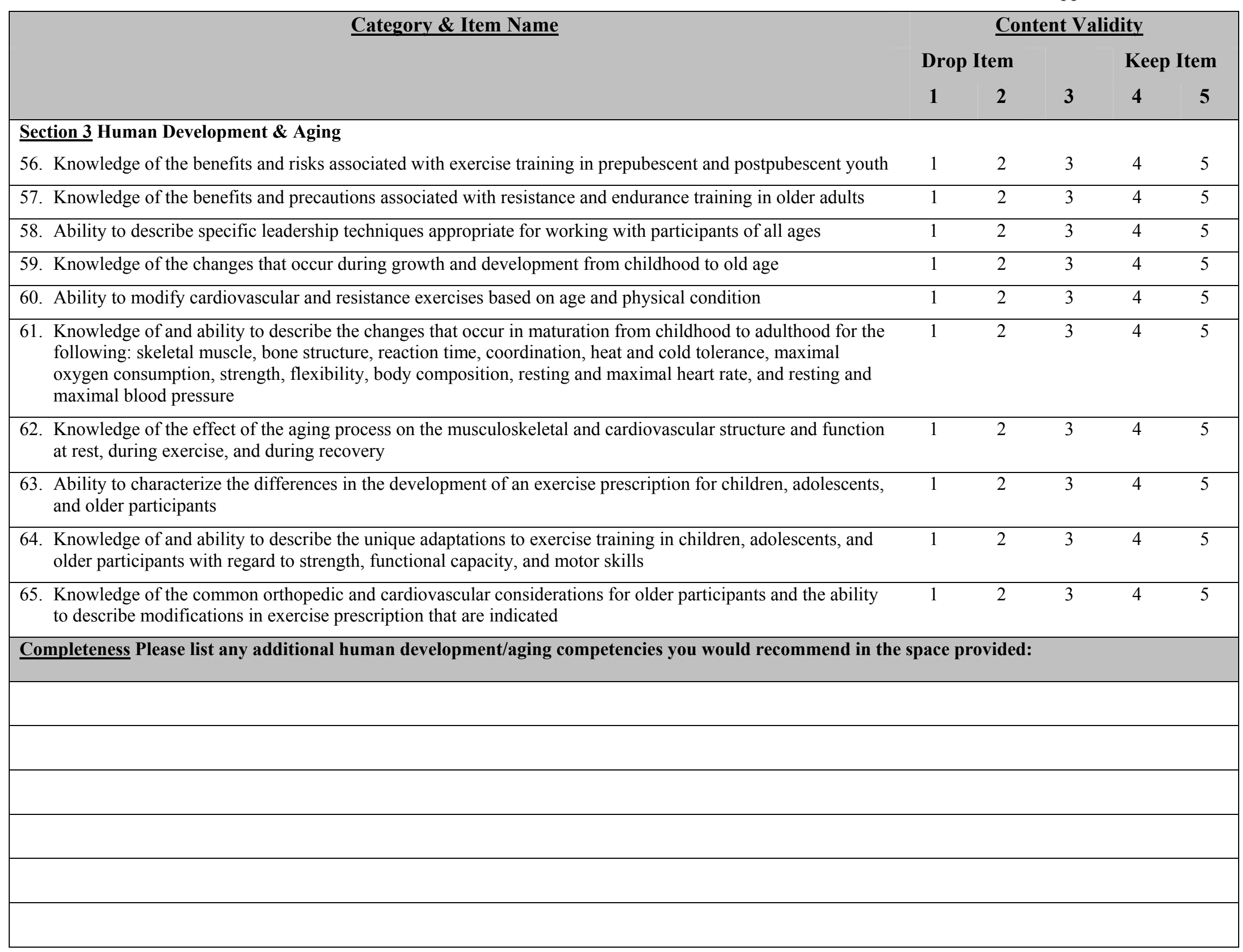




\begin{tabular}{|c|c|c|c|c|c|}
\hline \multirow[t]{3}{*}{$\underline{\text { Category \& Item Name }}$} & \multicolumn{5}{|c|}{ Content Validity } \\
\hline & \multicolumn{2}{|c|}{ Drop Item } & \multirow[b]{2}{*}{3} & \multicolumn{2}{|c|}{ Keep Item } \\
\hline & 1 & 2 & & 4 & 5 \\
\hline \multicolumn{6}{|l|}{ Section 4 Pathophysiology/Risk Factors } \\
\hline $\begin{array}{l}\text { 66. Knowledge of cardiovascular, respiratory, metabolic, and musculoskeletal risk factors that may require further } \\
\text { evaluation by medical or allied health professionals before participation in physical activity }\end{array}$ & 1 & 2 & 3 & 4 & 5 \\
\hline 67. Ability to determine those risk factors that may be favorably modified by physical activity habits & 1 & 2 & 3 & 4 & 5 \\
\hline $\begin{array}{l}\text { 68. Knowledge to define the following terms: total cholesterol (TC), high-density lipoprotein cholesterol (HDL- } \\
\text { C), TC/HDL-C ratio, low-density lipoprotein cholesterol (LDL-C), triglycerides, hypertension, and } \\
\text { artherosclerosis }\end{array}$ & 1 & 2 & 3 & 4 & 5 \\
\hline $\begin{array}{l}\text { 69. Knowledge of plasma cholesterol levels for adults as recommended by the National Cholesterol Education } \\
\text { Program (NCEP II) }\end{array}$ & 1 & 2 & 3 & 4 & 5 \\
\hline 70. Knowledge of the pathophysiology of atherosclerosis and how this process is influenced by physical activity & 1 & 2 & 3 & 4 & 5 \\
\hline $\begin{array}{l}\text { 71. Knowledge of the risk factor concept of coronary artery disease (CAD) and the influence of heredity and } \\
\text { lifestyle on the development of CAD }\end{array}$ & 1 & 2 & 3 & 4 & 5 \\
\hline $\begin{array}{l}\text { 72. Knowledge of the artherosclerotic process, the factors involved in its genesis and progression, and the } \\
\text { potential role of exercise training in treatment }\end{array}$ & 1 & 2 & 3 & 4 & 5 \\
\hline $\begin{array}{l}\text { 73. Ability to discuss in detail how lifestyle factors, including nutrition, physical activity, and heredity, influence } \\
\text { lipid and lipoprotein profiles }\end{array}$ & 1 & 2 & 3 & 4 & 5 \\
\hline $\begin{array}{l}\text { 74. Knowledge of cardiovascular risk factors or conditions that may require consultation with medical personnel } \\
\text { before testing or training, including inappropriate changes in resting or exercise heart rate and blood pressure, } \\
\text { new onset discomfort in chest, neck, shoulder, or arm, changes in the pattern of discomfort during rest or } \\
\text { exercise, fainting or dizzy spells, and claudication }\end{array}$ & 1 & 2 & 3 & 4 & 5 \\
\hline $\begin{array}{l}\text { 75. Knowledge of the respiratory risk factors or conditions that may require consultation with medical personnel } \\
\text { before testing or training, including asthma, exercise-induced bronchospasm, extreme breathlessness at rest or } \\
\text { during exercise, bronchitis, and emphysema }\end{array}$ & 1 & 2 & 3 & 4 & 5 \\
\hline $\begin{array}{l}\text { 76. Knowledge of the metabolic risk factors or conditions that may require consultation with medical personnel } \\
\text { before testing or training, including bodyweight by more than } 20 \% \text { above optimal, BMI }>30 \text {, thyroid disease, } \\
\text { diabetes or glucose intolerance, and hypoglycemia }\end{array}$ & 1 & 2 & 3 & 4 & 5 \\
\hline $\begin{array}{l}\text { 77. Knowledge of the musculoskeletal risk factors or conditions that may require consultation with medical } \\
\text { personnel before testing or training, including acute or chronic back pain, osteoarthritis, rheumatoid arthritis, } \\
\text { osteoporosis, tendonitis, and low back pain }\end{array}$ & 1 & 2 & 3 & 4 & 5 \\
\hline
\end{tabular}


Completeness Please list any additional pathophysiology/risk factor competencies you would recommend in the space provided:

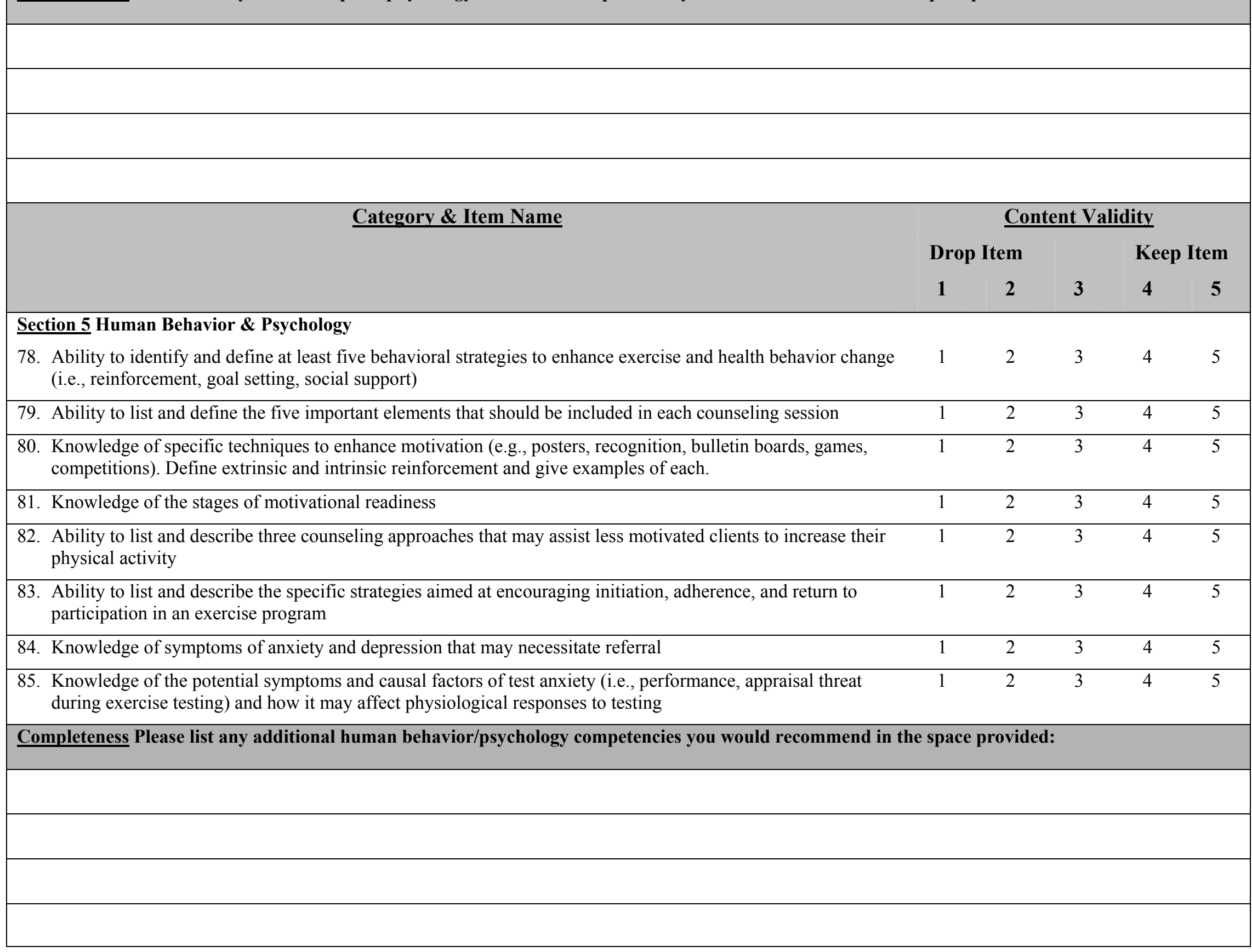




\begin{tabular}{|c|c|c|c|c|c|}
\hline \multirow[t]{3}{*}{ Category \& Item Name } & \multicolumn{5}{|c|}{ Content Validity } \\
\hline & \multicolumn{2}{|c|}{ Drop Item } & \multirow[b]{2}{*}{3} & \multicolumn{2}{|c|}{ Keep Item } \\
\hline & 1 & 2 & & 4 & 5 \\
\hline \multicolumn{6}{|l|}{ Section 6 Health Appraisal \& Fitness Testing } \\
\hline 86. Knowledge of the importance of a health/medical history & 1 & 2 & 3 & 4 & 5 \\
\hline 87. Knowledge of the value of a medical clearance prior to exercise participation & 1 & 2 & 3 & 4 & 5 \\
\hline 88. Skill to measure pulse rate accurately both at rest and during exercise & 1 & 2 & 3 & 4 & 5 \\
\hline $\begin{array}{l}\text { 89. Knowledge, skills, and abilities to assess the health status of individuals and the ability to conduct fitness } \\
\text { testing }\end{array}$ & 1 & 2 & 3 & 4 & 5 \\
\hline $\begin{array}{l}\text { 90. Ability to obtain a health history and risk appraisal that includes past and current medical history, family } \\
\text { history of cardiac disease, orthopedic limitations, prescribed medications, activity patterns, nutritional habits, } \\
\text { stress and anxiety levels, and smoking and alcohol use }\end{array}$ & 1 & 2 & 3 & 4 & 5 \\
\hline $\begin{array}{l}\text { 91. Ability to describe the categories of participants who should receive medical clearance prior to administration } \\
\text { of an exercise test or participation in an exercise program }\end{array}$ & 1 & 2 & 3 & 4 & 5 \\
\hline 92. Ability to identify relative and absolute contraindications to exercise testing or participation & 1 & 2 & 3 & 4 & 5 \\
\hline 93. Ability to discuss the limitations of informed consent and medical clearance prior to exercise testing & 1 & 2 & 3 & 4 & 5 \\
\hline 94. Ability to obtain informed consent & 1 & 2 & 3 & 4 & 5 \\
\hline $\begin{array}{l}\text { 95. Ability to explain the purpose and procedures for monitoring clients prior to, during, and after } \\
\text { cardiorespiratory fitness testing }\end{array}$ & 1 & 2 & 3 & 4 & 5 \\
\hline 96. Skill in instructing participants in the use of equipment and test procedures & 1 & 2 & 3 & 4 & 5 \\
\hline $\begin{array}{l}\text { 97. Ability to describe the purpose of testing, select an appropriate submaximal or maximal protocol, and conduct } \\
\text { an assessment of cardiovascular fitness on the cycle ergometer or the treadmill }\end{array}$ & 1 & 2 & 3 & 4 & 5 \\
\hline $\begin{array}{l}\text { 98. Skill in accurately measuring heart rate, blood pressure, and obtaining rating of perceived exertion (RPE) at } \\
\text { rest and during exercise according to established guidelines }\end{array}$ & 1 & 2 & 3 & 4 & 5 \\
\hline $\begin{array}{l}\text { 99. Ability to locate and measure skinfold sites, skeletal diameters, and girth measurements used for estimating } \\
\text { body composition }\end{array}$ & 1 & 2 & 3 & 4 & 5 \\
\hline $\begin{array}{l}\text { 100. Ability to describe the purpose of testing, select appropriate protocols, and conduct assessments of muscular } \\
\text { strength, muscular endurance, and flexibility }\end{array}$ & 1 & 2 & 3 & 4 & 5 \\
\hline 101.Skill in various techniques of assessing body composition & 1 & 2 & 3 & 4 & 5 \\
\hline 102.Knowledge of the advantages/disadvantages and limitations of the various body composition techniques & 1 & 2 & 3 & 4 & 5 \\
\hline $\begin{array}{l}\text { 103. Ability to interpret information obtained from cardiorespiratory fitness tests and muscular strength/endurance, } \\
\text { flexibility, and body composition tests for apparently healthy individuals and those with stable disease }\end{array}$ & 1 & 2 & 3 & 4 & 5 \\
\hline
\end{tabular}


Appendix A 128

\begin{tabular}{|lllll}
\hline $\begin{array}{l}\text { 104. Ability to identify appropriate criteria for terminating a fitness evaluation and demonstrate proper procedures } \\
\text { to be followed after discontinuing such a test }\end{array}$ & 1 & 2 & 3 \\
\hline $\begin{array}{c}\text { 105. Ability to modify protocols and procedures for cardiorespiratory fitness tests in children, adolescents, and } \\
\text { older adults }\end{array}$ & 1 & 2 & 3 & 5
\end{tabular}

Knowledge of common drugs from each of the following classes of medications and describe the principal action and the effects on exercise testing and prescription:

106.Antianginals $\quad \begin{array}{llll}2 & 3\end{array}$

107.Antihypertensives $\quad 3 \quad 2 \quad 3$

108.Antiarrhythmics $\quad 3 \quad 4 \quad 2$

\begin{tabular}{llll}
\hline 109. Bronchodilators & 3
\end{tabular}

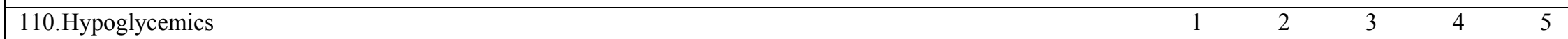

\begin{tabular}{lllll}
\hline 111. Psychotropics & 2 & 4 & 4
\end{tabular}

\begin{tabular}{lllll}
\hline 112. Vasodilators & 4 & 2 & 4
\end{tabular}

113.Ability to identify the effects of the following substances on exercise response: antihistamines, tranquilizers, 142 alcohol, diet pills, cold tablets, caffeine, and nicotine

114.Skill in techniques for calibration of a cycle ergometer and a motor-driven treadmill $3 \quad 4 \quad 2$

Completeness Please list any additional health appraisal/fitness testing competencies you would recommend in the space provided: 


\begin{tabular}{|c|c|c|c|c|c|}
\hline \multirow[t]{3}{*}{ Category \& Item Name } & \multicolumn{5}{|c|}{ Content Validity } \\
\hline & \multicolumn{2}{|c|}{ Drop Item } & \multirow[b]{2}{*}{3} & \multicolumn{2}{|c|}{ Keep Item } \\
\hline & 1 & 2 & & 4 & 5 \\
\hline \multicolumn{6}{|l|}{ Section 7 Safety, Injury Prevention, and Emergency Care } \\
\hline 115.Knowledge of and skill in obtaining basic life support and cardiopulmonary certification & 1 & 2 & 3 & 4 & 5 \\
\hline $\begin{array}{l}\text { 116.Knowledge of appropriate emergency procedures (i.e., telephone procedures, written emergency procedures, } \\
\text { personnel responsibilities) in the group exercise setting }\end{array}$ & 1 & 2 & 3 & 4 & 5 \\
\hline $\begin{array}{l}\text { 117. Knowledge of basic first aid procedures for exercise-related injuries, such as bleeding, strains/sprains, } \\
\text { fractures, and exercise intolerance (dizziness, syncope, heat injury) }\end{array}$ & 1 & 2 & 3 & 4 & 5 \\
\hline 118. Knowledge of basic precautions taken in a group exercise setting to ensure participant safety & 1 & 2 & 3 & 4 & 5 \\
\hline 119.Ability to identify the physical and physiological signs and symptoms of overtraining & 1 & 2 & 3 & 4 & 5 \\
\hline 120.Ability to list the effects of temperature, humidity, altitude, and pollution on physiological response to exercise & 1 & 2 & 3 & 4 & 5 \\
\hline $\begin{array}{l}\text { 121.Knowledge of the following terms: shin splints, sprain, strain, tennis elbow, bursitis, stress fracture, tendonitis, } \\
\text { patellar femoral pain syndrome, low back pain, plantar fasciitis, and rotator cuff tendonitis }\end{array}$ & 1 & 2 & 3 & 4 & 5 \\
\hline 122.Skill to demonstrate exercises used for people with low back pain & 1 & 2 & 3 & 4 & 5 \\
\hline $\begin{array}{l}\text { 123. Knowledge of hypothetical concerns and potential risks that may be associated with the use of exercises such } \\
\text { as straight leg sit-ups, double leg raises, full squats, hurdlers stretch, yoga plough, forceful back } \\
\text { hyperextension, and standing bent-over toe touch }\end{array}$ & 1 & 2 & 3 & 4 & 5 \\
\hline 124.Skill in demonstrating appropriate emergency procedures during exercise testing and/or training & 1 & 2 & 3 & 4 & 5 \\
\hline $\begin{array}{l}\text { 125. Knowledge of safety plans, emergency procedures, and first aid techniques needed during fitness evaluations, } \\
\text { exercise testing, and exercise training }\end{array}$ & 1 & 2 & 3 & 4 & 5 \\
\hline 126. Ability to identify the components that contribute to the maintenance of a safe environment & 1 & 2 & 3 & 4 & 5 \\
\hline $\begin{array}{l}\text { 127. Knowledge of the health/fitness instructor's responsibilities, limitations, and the legal implications of carrying } \\
\text { out emergency procedures }\end{array}$ & 1 & 2 & 3 & 4 & 5 \\
\hline $\begin{array}{l}\text { 128. Ability to describe potential musculoskeletal injuries (e.g., contusions, sprains, strains, fractures), } \\
\text { cardiovascular/pulmonary complications (e.g., tachycardia, bradycardia, hypotension/hypertension, } \\
\text { tachypnea), and metabolic abnormalities (e.g., fainting/syncope, hypoglycemia/hyperglycemia, } \\
\text { hypothermia/hyperthermia) }\end{array}$ & 1 & 2 & 3 & 4 & 5 \\
\hline $\begin{array}{l}\text { 129. Knowledge of the initial management and first aid techniques associated with open wounds, musculoskeletal } \\
\text { injuries, cardiovascular/pulmonary complications, and metabolic disorders }\end{array}$ & 1 & 2 & 3 & 4 & 5 \\
\hline $\begin{array}{l}\text { 130. Knowledge of the components of an equipment maintenance/repair program and how it may be used to } \\
\text { evaluate the condition of exercise equipment to reduce potential risk of injury }\end{array}$ & 1 & 2 & 3 & 4 & 5 \\
\hline
\end{tabular}


Completeness Please list any additional safety/injury prevent/emergency competencies you would recommend in the space provided:

\begin{tabular}{|l|}
\hline \\
\hline \\
\hline \\
\hline \\
\hline \\
\hline \\
\hline \\
\hline \\
\hline
\end{tabular}


Drop Item

12

\section{Section 8 Exercise Programming}

131. Knowledge of the recommended intensity, duration, frequency, and type of physical activity necessary for development of cardiorespiratory fitness in an apparently healthy population

132. Ability to differentiate between the amount of physical activity required for health benefits and the amount of exercise required for fitness development

133.Ability to describe exercises designed to enhance muscular strength and/or endurance of specific major muscle 1 groups

134.Knowledge of the principles of overload, specificity, and progression and how they relate to exercise programming

135.Skill to teach and demonstrate appropriate exercises used in the warm-up and cool-down of a variety of group exercise classes

136. Ability to teach the components of an exercise session (i.e., warm-up, aerobic stimulus phase, cool-down, muscular strength/endurance, flexibility)

137.Knowledge of the following terms: progressive resistance, isotonic/isometric, concentric, eccentric, atrophy, 14 hypertrophy, sets, repetitions, plyometrics, Valsalva maneuver

138. Skill to teach class participants how to monitor intensity of exercise using heart rate and rating of perceived 34 exertion (RPE)

139. Skill to teach participants how to use RPE and heart rate to adjust the intensity of the exercise session

140.Ability to calculate training heart rates using two methods: percent of age-predicted maximum heart rate and heart rate reserve (Karvonen)

141.Skill to teach and demonstrate appropriate modifications in specific exercises for the following groups: older $1 \quad 2 \quad 3$ adults, pregnant and postnatal women, obese persons, and persons with low back pain

142.Ability to recognize proper and improper techniques in the use of resistive equipment such a stability balls, $\quad 1 \quad 2 \quad 2$ weights, bands, resistance bars, and water exercise equipment

143.Ability to recognize proper and improper technique in the use of cardiovascular conditioning equipment (e.g., $1 \quad 3 \quad 2$ steps, cycles, slides)

144. Skill to teach and demonstrate appropriate exercises for improving range of motion of all major joints

145.Ability to modify exercises in the group setting for apparently healthy persons of various fitness levels

146. Ability to teach a progression of exercises for all major muscle groups to improve muscular strength and endurance 
Appendix A 132

\begin{tabular}{|c|c|c|c|c|c|}
\hline 147. Knowledge to describe the various types of interval, continuous, and circuit training programs & 1 & 2 & 3 & 4 & 5 \\
\hline $\begin{array}{l}\text { 148. Knowledge to describe various ways a leader can take a position relative to the group to enhance visibility, } \\
\text { participant interactions, and communication }\end{array}$ & 1 & 2 & 3 & 4 & 5 \\
\hline 149.Ability to communicate effectively with exercise participants in the group exercise session & 1 & 2 & 3 & 4 & 5 \\
\hline 150.Knowledge to describe partner resistance exercises that can be used in a group class setting & 1 & 2 & 3 & 4 & 5 \\
\hline 151.Ability to demonstrate techniques for accommodating various fitness levels within the same class & 1 & 2 & 3 & 4 & 5 \\
\hline 152.Knowledge of the properties of water that affect the design of a water exercise session & 1 & 2 & 3 & 4 & 5 \\
\hline 153. Knowledge of basic music fundamentals, including downbeat, 8 count, and 32 count & 1 & 2 & 3 & 4 & 5 \\
\hline $\begin{array}{l}\text { 154. Skill to effectively use verbal and nonverbal cues in the group exercise setting, including anticipatory, } \\
\text { motivational, safety, and educational }\end{array}$ & 1 & 2 & 3 & 4 & 5 \\
\hline $\begin{array}{l}\text { 155. Skill to demonstrate the proper form, alignment, and technique in typical exercises used in warm-up, stimulus, } \\
\text { muscle conditioning, and cool-down phases of the group session }\end{array}$ & 1 & 2 & 3 & 4 & 5 \\
\hline 156. Ability to evaluate specific exercises in terms of safety and effectiveness for various participants & 1 & 2 & 3 & 4 & 5 \\
\hline $\begin{array}{l}\text { 157. Ability to demonstrate a familiarity with a variety of group exercise formats (e.g., traditional, step, slide, } \\
\text { muscle conditioning, flexibility, indoor cycling, water fitness, walking) }\end{array}$ & 1 & 2 & 3 & 4 & 5 \\
\hline $\begin{array}{l}\text { 158. Knowledge, skills, and abilities to prescribe and administer exercise programs for apparently healthy } \\
\text { individuals, individuals at higher risk, and individuals with known disease }\end{array}$ & 1 & 2 & 3 & 4 & 5 \\
\hline $\begin{array}{l}\text { 159. Ability to design, implement, and evaluate individualized and group exercise programs based on health history } \\
\text { and physical fitness assessments }\end{array}$ & 1 & 2 & 3 & 4 & 5 \\
\hline 160.Ability to modify exercises based on age and physical condition & 1 & 2 & 3 & 4 & 5 \\
\hline $\begin{array}{l}\text { 161. Knowledge, skills, and abilities to calculate energy cost, } \mathrm{VO} 2, \mathrm{MET} \text { s, and target heart rates and apply the } \\
\text { information to an exercise prescription }\end{array}$ & 1 & 2 & 3 & 4 & 5 \\
\hline $\begin{array}{l}\text { 162. Ability to convert weights from pounds }(\mathrm{lb}) \text { to kilograms }(\mathrm{kg}) \text { and speed from miles per hour }(\mathrm{MPH}) \text { to meters } \\
\text { per minute }(\mathrm{m} \cdot \mathrm{min}-1)\end{array}$ & 1 & 2 & 3 & 4 & 5 \\
\hline 163. Ability to convert METs to VO2 expressed as $\mathrm{mL} \cdot \mathrm{kg}-1 \cdot \min -1$, $\mathrm{L} \cdot \mathrm{min}-1$, and/or $\mathrm{mL} \cdot \mathrm{kg} \mathrm{FFW}-1 \cdot \min -1$ & 1 & 2 & 3 & 4 & 5 \\
\hline $\begin{array}{l}\text { 164. Ability to calculate the energy cost in METs and kilocalories for given exercise intensities in stepping exercise, } \\
\text { cycle ergometry, and during horizontal and graded walking and running }\end{array}$ & 1 & 2 & 3 & 4 & 5 \\
\hline 165.Knowledge of the approximate METs for various sport, recreational, and work tasks & 1 & 2 & 3 & 4 & 5 \\
\hline $\begin{array}{l}\text { 166. Ability to prescribe exercise intensity based on } \mathrm{VO} 2 \text { data for different modes of exercise, including graded and } \\
\text { horizontal running and walking, cycling, and stepping exercise }\end{array}$ & 1 & 2 & 3 & 4 & 5 \\
\hline $\begin{array}{l}\text { 167. Ability to explain and implement exercise prescription guidelines for apparently health clients, increased risk } \\
\text { clients, and clients with controlled disease }\end{array}$ & 1 & 2 & 3 & 4 & 5 \\
\hline
\end{tabular}




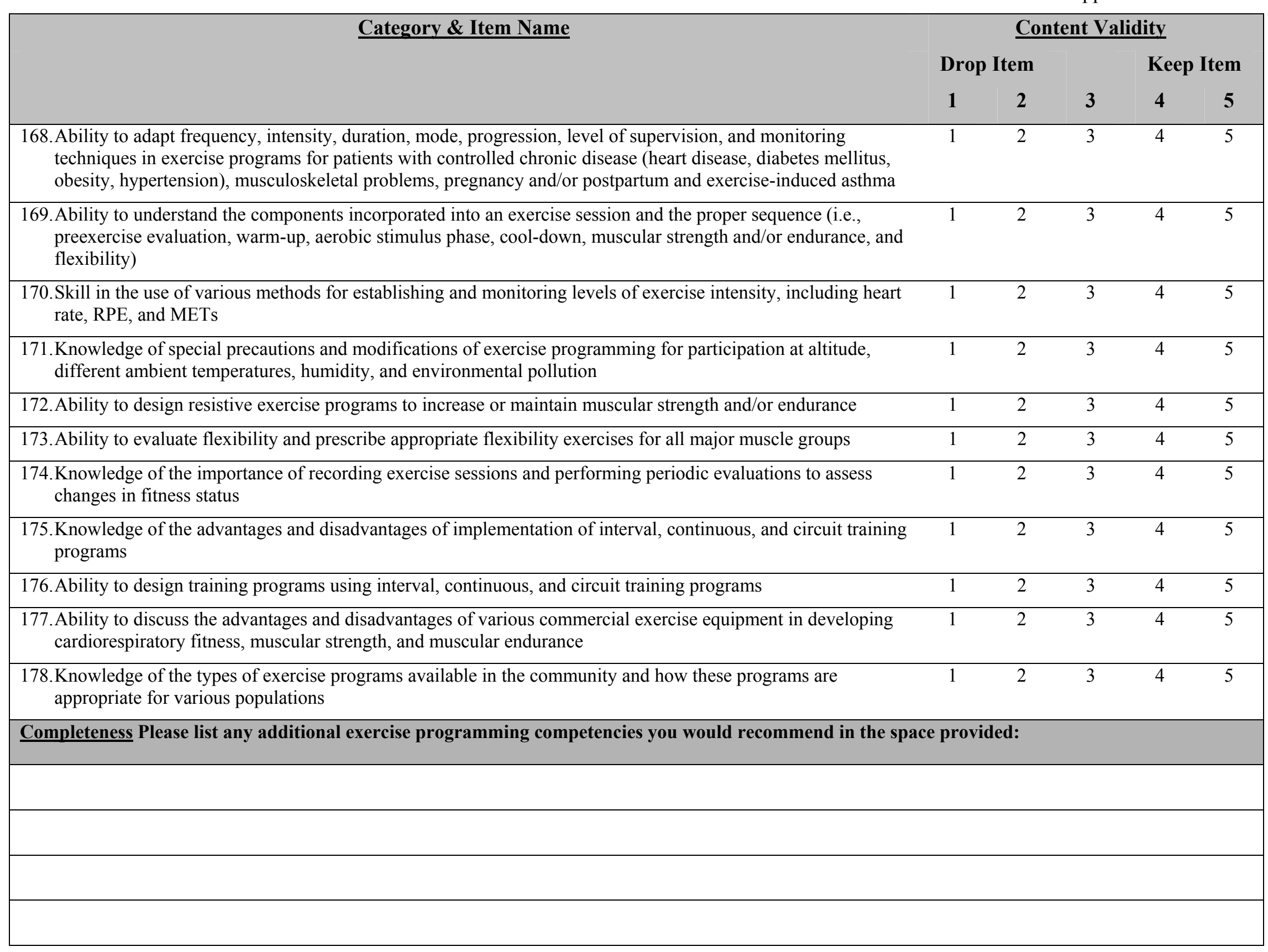




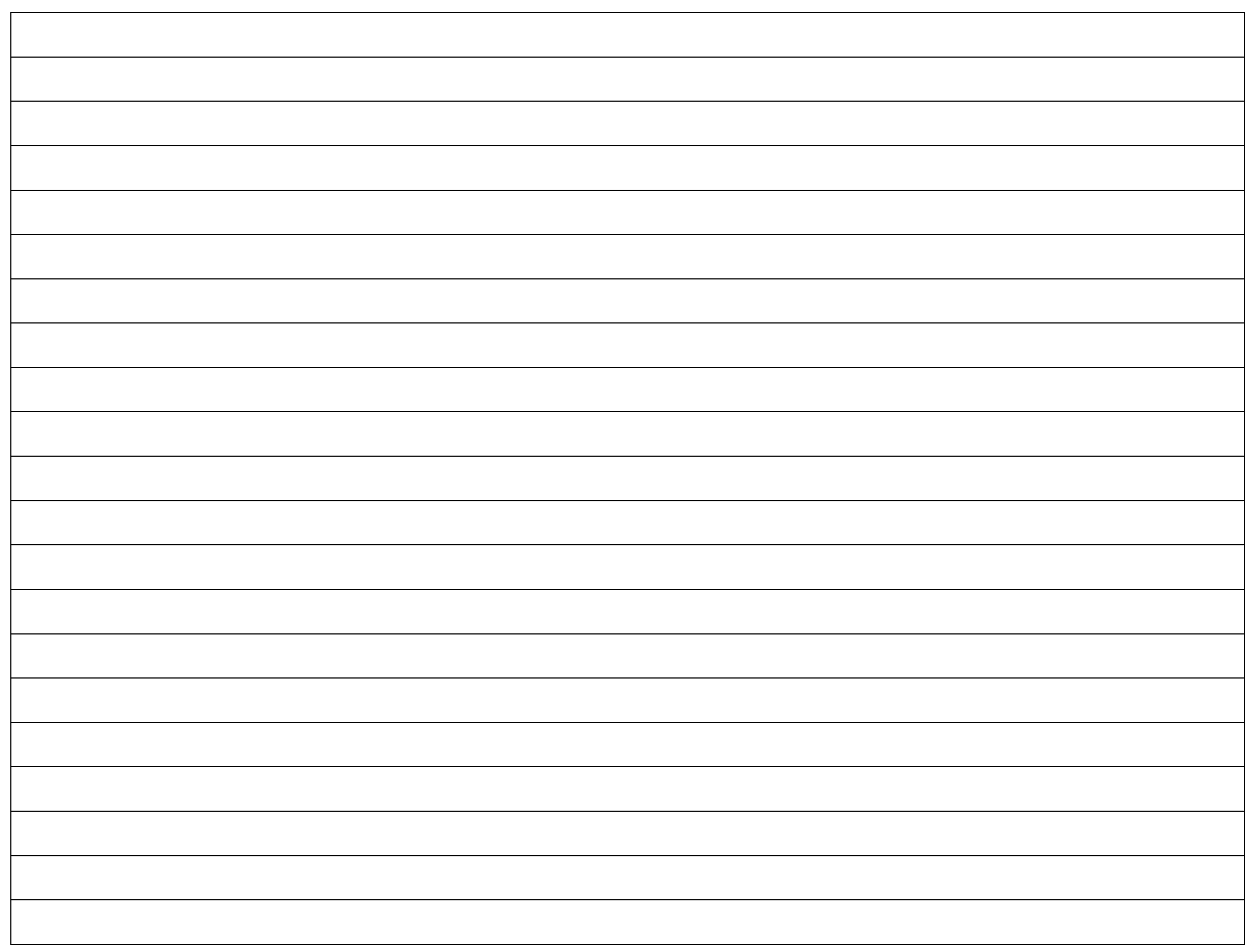




\begin{tabular}{|c|c|c|c|c|c|}
\hline \multirow{3}{*}{ Category \& Item Name } & \multicolumn{5}{|c|}{ Content Validity } \\
\hline & \multicolumn{2}{|c|}{ Drop Item } & \multirow[b]{2}{*}{3} & \multicolumn{2}{|c|}{ Keep Item } \\
\hline & 1 & 2 & & 4 & 5 \\
\hline \multicolumn{6}{|l|}{ Section 9 Nutrition \& Weight Management } \\
\hline $\begin{array}{l}\text { 179. Knowledge to define the following terms: obesity, overweight, percent fat, lean body mass, anorexia nervosa, } \\
\text { bulimia, and body fat distribution }\end{array}$ & 1 & 2 & 3 & 4 & 5 \\
\hline 180.Knowledge of the relationship between body composition and health & 1 & 2 & 3 & 4 & 5 \\
\hline $\begin{array}{l}\text { 181. Knowledge of the effects of diet plus exercise, diet alone, and exercise alone as methods for modifying body } \\
\text { composition }\end{array}$ & 1 & 2 & 3 & 4 & 5 \\
\hline 182. Knowledge of the importance of an adequate daily energy intake for healthy weight management & 1 & 2 & 3 & 4 & 5 \\
\hline 183. Ability to differentiate between fat soluble and water-soluble vitamins & 1 & 2 & 3 & 4 & 5 \\
\hline 184. Ability to describe the importance of maintaining normal hydration before, during, and after exercise & 1 & 2 & 3 & 4 & 5 \\
\hline 185.Knowledge of the USDA Food Pyramid & 1 & 2 & 3 & 4 & 5 \\
\hline 186. Knowledge of the importance of calcium and iron in women's health & 1 & 2 & 3 & 4 & 5 \\
\hline $\begin{array}{l}\text { 187. Ability to describe the myths and consequences associated with inappropriate weight loss methods (e.g., } \\
\text { saunas, vibrating belts, body wraps, electric simulators, sweat suits, fad diets) }\end{array}$ & 1 & 2 & 3 & 4 & 5 \\
\hline 188. Knowledge of the number of kilocalories in one gram of carbohydrate, fat, protein, and alcohol & 1 & 2 & 3 & 4 & 5 \\
\hline 189. Knowledge of the number of kilocalories equivalent to losing 1 pound of body fat & 1 & 2 & 3 & 4 & 5 \\
\hline $\begin{array}{l}\text { 190. Knowledge, skills, and abilities to provide information concerning nutrition and the role of diet and exercise } \\
\text { on body composition and weight control }\end{array}$ & 1 & 2 & 3 & 4 & 5 \\
\hline $\begin{array}{l}\text { 191. Ability to describe the health implications of variation in body fat distribution patterns and the significance of } \\
\text { waist to hip ratio }\end{array}$ & 1 & 2 & 3 & 4 & 5 \\
\hline 192. Knowledge of the guidelines for caloric intake for an individual desiring to lose or gain weight & 1 & 2 & 3 & 4 & 5 \\
\hline $\begin{array}{l}\text { 193. Knowledge of common nutritional ergogenic aids, the purported mechanism of action, and any risk and/or } \\
\text { benefit (e.g., carbohydrates, protein/amino acids, vitamins, minerals, sodium bicarbonate, creatine, bee pollen) }\end{array}$ & 1 & 2 & 3 & 4 & 5 \\
\hline $\begin{array}{l}\text { 194. Knowledge of nutritional factors related to the female athlete triad syndrome (i.e., eating disorders, menstrual } \\
\text { cycle abnormalities, and osteoporosis) }\end{array}$ & 1 & 2 & 3 & 4 & 5 \\
\hline $\begin{array}{l}\text { 195. Knowledge of the NIH Consensus statement regarding health risks of obesity, Nutrition for Physical Fitness } \\
\text { Position Paper of the American Dietetic Association, and the ACSM Position Stand on proper and improper } \\
\text { weight loss programs }\end{array}$ & 1 & 2 & 3 & 4 & 5 \\
\hline 196.Knowledge of NCEP II guidelines for lipid management & 1 & 2 & 3 & 4 & 5 \\
\hline
\end{tabular}


Completeness Please list any additional nutritional/weight management competencies you would recommend in the space provided:

\section{$\underline{\text { Category \& Item Name }}$}

\section{Content Validity}

Drop Item

Keep Item

1

\section{Section 10 Program \& Administration/Management}

197.Knowledge, skills, and ability to administer and deliver health/fitness programs

$\begin{array}{lllll}1 & 2 & 3 & 4 & 5 \\ 1 & 2 & 3 & 4 & 5\end{array}$

198.Knowledge of the health/fitness instructor's supportive role in administration and program management within a health fitness facility

199.Ability to administer fitness-related programs within established budgetary guidelines

$\begin{array}{lllll}1 & 2 & 3 & 4 & 5\end{array}$

200.Ability to develop marketing materials for the purpose of promoting fitness-related programs

$\begin{array}{lllll}1 & 2 & 3 & 4 & 5 \\ 1 & 2 & 3 & 4 & 5 \\ 1 & 2 & 3 & 4 & 5\end{array}$

201. Ability to use various sales techniques for prospective program clients/participants

202.Ability to describe and use the documentation required when a client shows signs or symptoms during an exercise session and should be referred to a physician

\begin{tabular}{|lllllll}
\hline 203.Ability to create and maintain records pertaining to participant exercise adherence, retention, and goal setting & 1 & 2 & 3 & 4 & 5
\end{tabular}

204.Ability to develop and administer educational programs (e.g., lectures, workshops) and educational materials

205.Knowledge of management of a fitness department (e.g., working within a budget, training exercise leaders, scheduling, running staff meetings)

206.Knowledge of the importance of tracking and evaluating member retention

$\begin{array}{lllll}1 & 2 & 3 & 4 & 5 \\ 1 & 2 & 3 & 4 & 5 \\ 1 & 2 & 3 & 4 & 5\end{array}$

Completeness Please list any additional program management competencies you would recommend in the space provided:

THANKS AGAIN FOR YOUR TIME \& CONSIDERATION 


\section{APPENDIX B}

Modified Delphi Investigation of Exercise Science

in Physical Education Teacher Education

Round I of the Study 


\section{PHONE SCRIPT FOR DELPHI PANEL MEMBER RECRUITMENT}

"Hello, my name is Sean Bulger and I am calling to request your participation as an expert panel member for my dissertation research titled a Modified Delphi Investigation of Exercise Science in Physical Education Teacher Education as partial fulfillment for the doctoral degree from the School of Physical Education at West Virginia University. The research will involve a Modified Delphi investigation to determine critical exercise science competencies that should be included in the physical education teacher education undergraduate curriculum. This investigative process will involve 2 rounds of questionnaire circulation. As a panel member, you will be asked to rate a list of exercise science concepts in terms of their importance and pedagogical relevance for prospective physical educators. Additionally, you will be asked to provide your recommended instructional strategies for the delivery of exercise science content in the physical education teacher education curriculum. Your expertise is essential for identifying this list of critical exercise science competencies and recommended instructional strategies. Are you interested in participating as an expert panel member for this study?"

\section{YES/NO (IF “NO” GO TO 1 / IF "YES” GO TO 2)}

1. "Thank you for your time and consideration."

2. "Thank you for agreeing to participate in this study, your time and cooperation are greatly appreciated. Within the next week, I will mail you an information packet that includes some additional details regarding your participation in this study and a copy of the first round questionnaire. How would you like me to address this mailing?" (RECORD MAILING ADDRESS IN THE SPACE PROVIDED BELOW) “Thanks again for agreeing to participate. Your expert contribution is critical to the success of this research project." 


\section{(ON LETTERHEAD STATIONARY)}

(PANEL MEMBER NAME)

(MAILING ADDRESS)

(CITY, STATE, ZIP CODE)

\section{(DATE)}

Dear

Thank you for agreeing to be a participant in my study titled a Modified Delphi Investigation of Exercise Science in Physical Education Teacher Education. As we discussed in our previous phone conversation, you were chosen because of your expertise in the area of exercise science as related to the preparation of prospective physical educators and/or its eventual application in school-based physical education programs.

The research will involve the use of a modified Delphi protocol to determine the critical exercise science competencies recommended for inclusion in the physical education teacher education undergraduate curriculum. This investigative process will include 2 rounds of questionnaire circulation. The Delphi panel will consist of 15 exercise science specialists, 10 teacher education specialists, and 5 practicing physical educators who have been identified as experts in the area of exercise science as related to the preparation of prospective physical educators and/or the instruction of physical education in school-based settings. As a panel member, you will be asked to rate a list of exercise science concepts in terms of their importance and pedagogical relevance for prospective physical educators.

The items that you are evaluating were adapted from the American College of Sports Medicine (2000) ${ }^{1}$ and modified with the permission of the copyright owner, Lippincott Williams \& Wilkins. Please read the instructions that have been provided prior to completing the survey. If you have any questions, contact me at (715) 836-3722 or bulgersm@uwec.edu.

The results of this research project will be used to fulfill the requirements for my doctoral dissertation, which I am completing at West Virginia University. Your participation in the research process is entirely voluntary and you do not need to respond to every item on the questionnaire. You may be assured of complete confidentiality regarding all of your responses. I will send you the final results of the study upon its completion.

If possible, please return the completed survey to me within three weeks of receiving it. Additionally, send a current copy of your curriculum vitae or resume if you would like it included in my dissertation as recognition of your contribution to the project. Use the return envelope that I have provided for your convenience and accept the enclosed gift as a small gesture of my appreciation for sharing your time, effort, and expertise. Thanks again for your assistance and cooperation.

Sincerely,

Sean M. Bulger

${ }^{1}$ ACSM. (2000). ACSM's Guidelines for Exercise Testing and Prescription (6 $6^{\text {th }}$ edition). Baltimore, MD: Lippincott Williams \& Wilkins. 


\title{
Modified Delphi Investigation of Exercise Science in Physical Education Teacher Education
}

\author{
Round I Questionnaire
}

September 2003

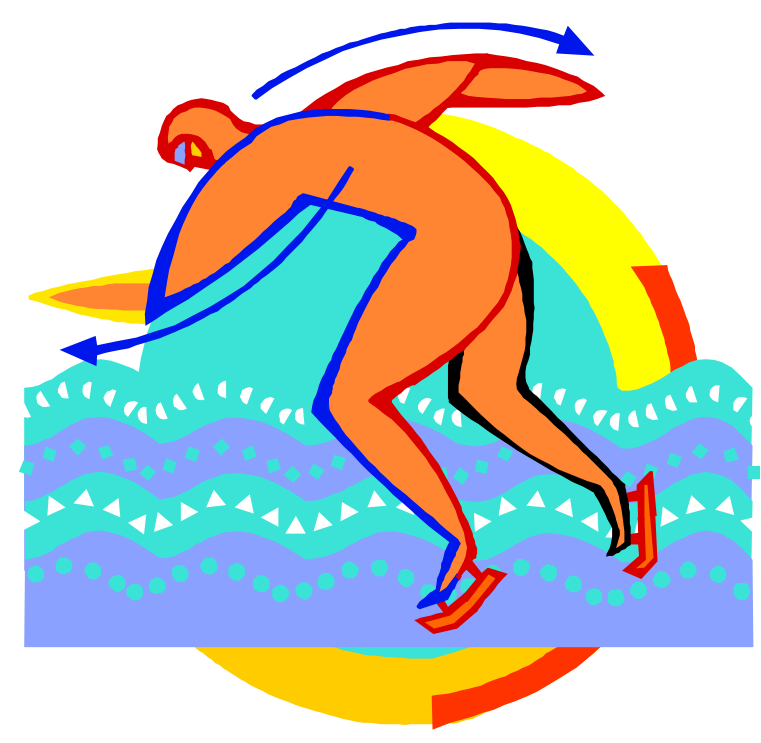

Sean M. Bulger, Doctoral Candidate

West Virginia University

School of Physical Education 


\section{Introduction}

The questionnaire you have agreed to complete will be used to generate a consensus of expert opinion regarding the critical theoretical and applied exercise science competencies that prospective physical educators need to learn within the physical education teacher education curriculum. The Delphi Method will be employed to address this primary research question. The Delphi Method is a research protocol that involves (a) the identification and selection of a small panel of experts on a particular topic, (b) the use of multiple rounds of surveying as a means to collect expert opinion, and (c) the attainment of group consensus through the provision of regular feedback.

Your expertise and judgment remains central to the success of this project. Due to the nature of both the involved research question and the Delphi Method, the list of potential survey items I am asking you to evaluate is rather extensive. The items that you are evaluating were adapted and modified from the American College of Sports Medicine (2000) ${ }^{1}$ and reproduced with the permission of the copyright owner, Lippincott Williams \& Wilkins.

Please know that your commitment to the timely completion of this project is greatly appreciated.

\section{Table of Contents}

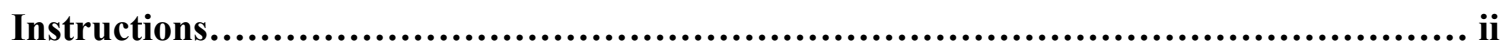

Section 1: Anatomy \& Biomechanics (Items 1-23) .................................... 1

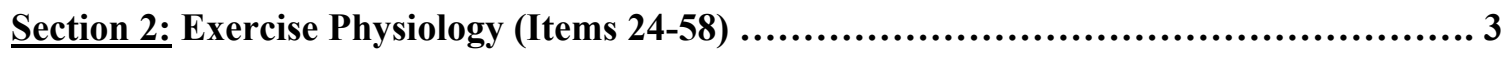

Section 3: Human Development \& Aging (Items 59-69) ................................. 6

Section 4: Pathophysiology/Risk Factors (Items 70-83) ............................... 7

Section 5: Human Behavior \& Psychology (Items 84-100) ................................ 9

Section 6: Health Appraisal \& Fitness Testing (Items 101-132) ............................. 11

Section 7: Safety, Injury Prevention, and Emergency Care (Items 133-148) ................. 13

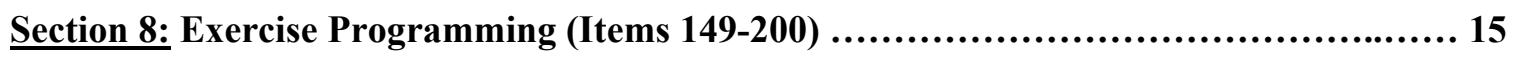

Section 9: Nutrition \& Weight Management (Items 201-218) ............................ 19

Section 10: Program \& Administration/Management (Items 219-222) ................... 21

\footnotetext{
${ }^{1}$ ACSM. (2000). ACSM's Guidelines for Exercise Testing and Prescription (6 ${ }^{\text {th }}$ edition). Baltimore, MD: Lippincott
} Williams \& Wilkins. 


\section{To: Exercise Science Delphi Panel Members \\ From: Sean M. Bulger, Doctoral Candidate \\ RE: Instructions for Completing the Round I Survey Instrument}

Again, thank you very much for your assistance in completing this research project. It is hoped that this study will result in the generation of a comprehensive list of critical theoretical and applied exercise science competencies that are recommended for inclusion in the physical education teacher education curriculum. Using the following set of instructions, please complete the attached questionnaire and return it to me within the next three weeks.

Rate each of the questionnaire items separately in the areas of IMPORTANCE and RELEVANCE. For the purpose of this study, IMPORTANCE will refer to the item's value as a theoretical underpinning of the physical education profession. RELEVANCE will refer to the item's practical or applied value to the K-12 physical educator.

A rating of (5) or (4) in BOTH IMPORTANCE and RELEVANCE means that you consider the item to be critical in the preparation of prospective K-12 physical education teachers.

A rating of (3) means you are neutral or undecided regarding the IMPORTANCE and RELEVANCE of the item.

$\square$ A rating of (2) or (1) on EITHER IMPORTANCE or RELEVANCE means the item is not critical for the preparation of prospective K-12 physical education teachers.

$\square$ If you choose not to respond to a particular item, please leave it blank.

When all Round I surveys have been received and analyzed, I will mail you the Round II instructions and questionnaire. The Round II questionnaire will include a summary of the responses from the Round I questionnaire for your further consideration.

\section{Thanks again and please feel free to contact me directly with any questions at (715) 836-3722 or bulgersm@uwec.edu}

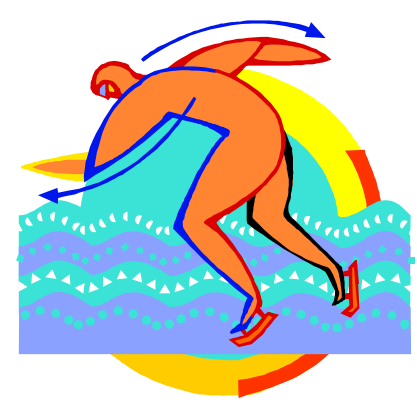




\section{Modified Delphi Investigation of Exercise Science in Physical Education Teacher Education} Round I Questionnaire

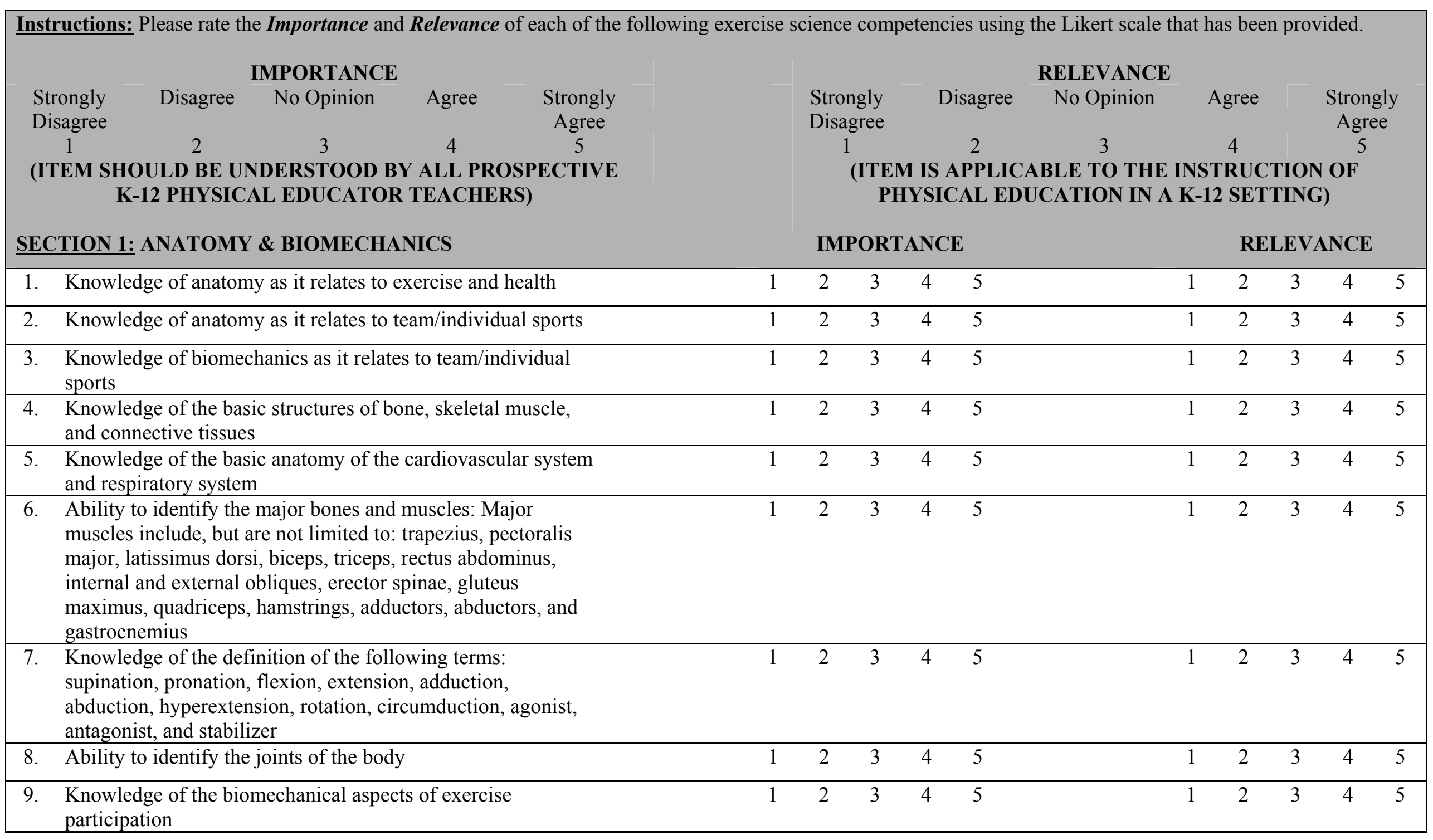




\begin{tabular}{|c|c|c|c|c|c|c|c|c|c|c|}
\hline $\begin{array}{l}\text { 10. Knowledge to identify the plane in which each muscle action } \\
\text { occurs }\end{array}$ & 1 & 2 & 3 & 4 & 5 & 1 & 2 & 3 & 4 & 5 \\
\hline $\begin{array}{l}\text { 11. Knowledge of the interrelationships among center of gravity, } \\
\text { base of support, balance, stability, and proper spinal } \\
\text { alignment }\end{array}$ & 1 & 2 & 3 & 4 & 5 & 1 & 2 & 3 & 4 & 5 \\
\hline $\begin{array}{l}\text { 12. Ability to describe the following curvatures of the spine: } \\
\text { lordosis, scoliosis, and kyphosis }\end{array}$ & 1 & 2 & 3 & 4 & 5 & 1 & 2 & 3 & 4 & 5 \\
\hline $\begin{array}{l}\text { 13. Knowledge of and skill to demonstrate exercises designed to } \\
\text { enhance muscular strength and/or endurance of specific major } \\
\text { muscle groups }\end{array}$ & 1 & 2 & 3 & 4 & 5 & 1 & 2 & 3 & 4 & 5 \\
\hline $\begin{array}{l}\text { 14. Knowledge of and skill to demonstrate exercise for enhancing } \\
\text { musculoskeletal flexibility }\end{array}$ & 1 & 2 & 3 & 4 & 5 & 1 & 2 & 3 & 4 & 5 \\
\hline 15. Knowledge to describe the myotatic stretch reflex & 1 & 2 & 3 & 4 & 5 & 1 & 2 & 3 & 4 & 5 \\
\hline $\begin{array}{l}\text { 16. Knowledge to identify the primary action and joint range of } \\
\text { motion for each major muscle group }\end{array}$ & 1 & 2 & 3 & 4 & 5 & 1 & 2 & 3 & 4 & 5 \\
\hline 17. Knowledge of functional anatomy and biomechanics & 1 & 2 & 3 & 4 & 5 & 1 & 2 & 3 & 4 & 5 \\
\hline $\begin{array}{l}\text { 18. Knowledge of the structure and ability to describe movements } \\
\text { for the major joints of the body }\end{array}$ & 1 & 2 & 3 & 4 & 5 & 1 & 2 & 3 & 4 & 5 \\
\hline $\begin{array}{l}\text { 19. Ability to locate the anatomic landmarks for palpation of } \\
\text { peripheral pulses }\end{array}$ & 1 & 2 & 3 & 4 & 5 & 1 & 2 & 3 & 4 & 5 \\
\hline $\begin{array}{l}\text { 20. Ability to locate the brachial artery and correctly place the } \\
\text { cuff and stethoscope in position for blood pressure } \\
\text { measurement }\end{array}$ & 1 & 2 & 3 & 4 & 5 & 1 & 2 & 3 & 4 & 5 \\
\hline $\begin{array}{l}\text { 21. Ability to locate common sites for measurement of skinfold } \\
\text { thickness and circumferences (for determination of body } \\
\text { composition and waist-hip ratio) }\end{array}$ & 1 & 2 & 3 & 4 & 5 & 1 & 2 & 3 & 4 & 5 \\
\hline $\begin{array}{l}\text { 22. Knowledge of the biomechanical principles that underlie } \\
\text { performance of the following activities: walking, jogging, } \\
\text { running, swimming, cycling, weight lifting, and carrying or } \\
\text { moving objects }\end{array}$ & 1 & 2 & 3 & 4 & 5 & 1 & 2 & 3 & 4 & 5 \\
\hline $\begin{array}{l}\text { 23. Knowledge of the influence of weight-bearing activity in } \\
\text { childhood and adolescence on optimal bone growth and } \\
\text { development }\end{array}$ & 1 & 2 & 3 & 4 & 5 & 1 & 2 & 3 & 4 & 5 \\
\hline
\end{tabular}




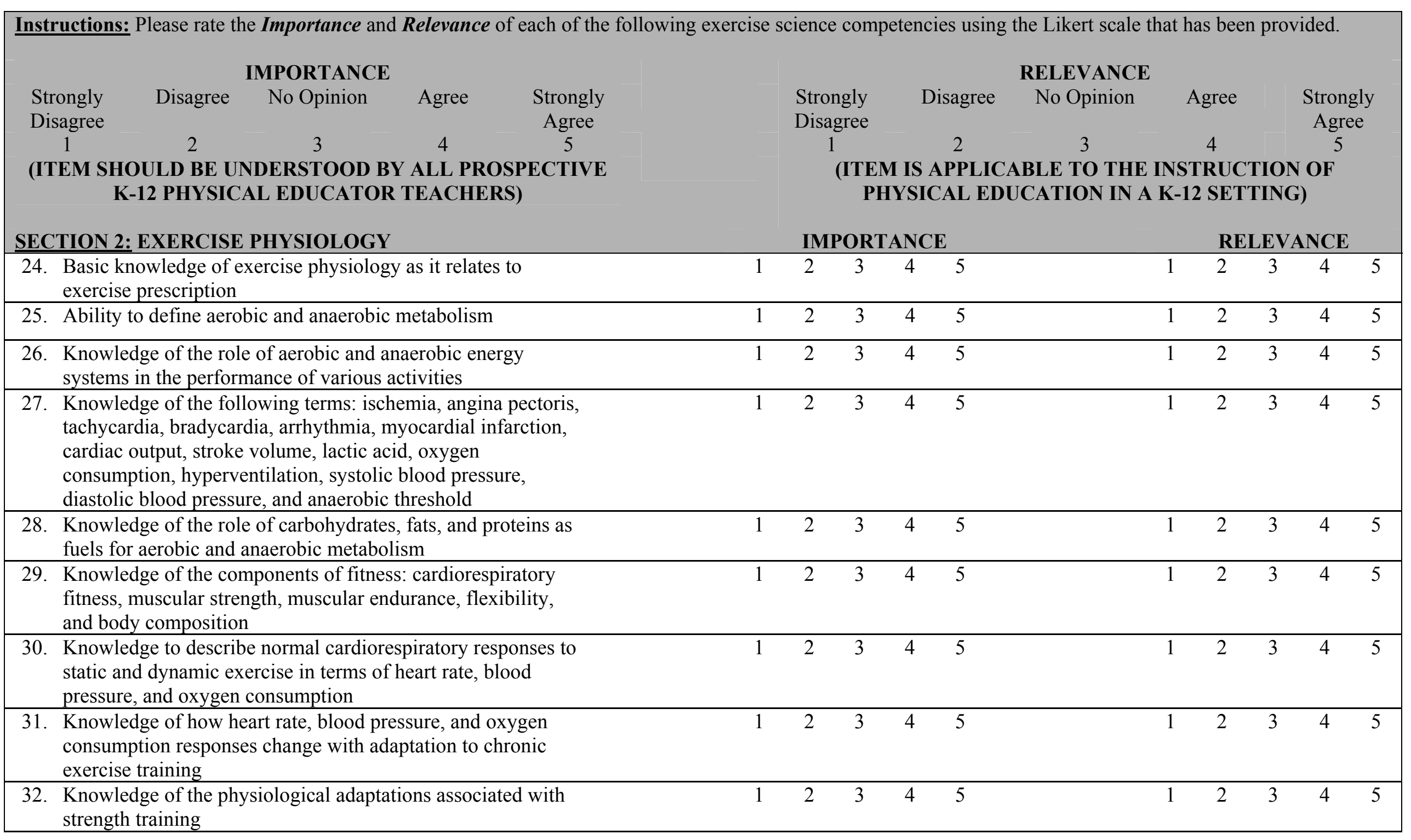




\begin{tabular}{|c|c|c|c|c|c|c|c|c|c|c|c|}
\hline 33. & $\begin{array}{l}\text { Ability to identify and apply to both groups and individuals } \\
\text { methods used to monitor exercise intensity, including heart } \\
\text { rate and rating of perceived exertion }\end{array}$ & 1 & 2 & 3 & 4 & 5 & 1 & 2 & 3 & 4 & 5 \\
\hline 34. & $\begin{array}{l}\text { Knowledge of the physiological principles related to warm-up } \\
\text { and cool-down }\end{array}$ & 1 & 2 & 3 & 4 & 5 & 1 & 2 & 3 & 4 & 5 \\
\hline 35. & $\begin{array}{l}\text { Knowledge of the common theories of muscle fatigue and } \\
\text { delayed onset muscle soreness (DOMS) }\end{array}$ & 1 & 2 & 3 & 4 & 5 & 1 & 2 & 3 & 4 & 5 \\
\hline 36. & $\begin{array}{l}\text { Knowledge of exercise physiology including the role of } \\
\text { aerobic and anaerobic metabolism, muscle physiology, } \\
\text { cardiovascular physiology, and respiratory physiology at rest } \\
\text { and during exercise. In addition, demonstrate an } \\
\text { understanding of the components of physical fitness, the } \\
\text { effects of aerobic and strength and/or resistance training on } \\
\text { the fitness components and the effects of chronic disease }\end{array}$ & 1 & 2 & 3 & 4 & 5 & 1 & 2 & 3 & 4 & 5 \\
\hline 37. & $\begin{array}{l}\text { Knowledge of the physiological adaptations that occur at rest } \\
\text { and during submaximal and maximal exercise following } \\
\text { chronic aerobic and anaerobic exercise training }\end{array}$ & 1 & 2 & 3 & 4 & 5 & 1 & 2 & 3 & 4 & 5 \\
\hline 38. & $\begin{array}{l}\text { Knowledge of the differences in cardiorespiratory response to } \\
\text { acute graded exercise between conditioned and unconditioned } \\
\text { individuals }\end{array}$ & 1 & 2 & 3 & 4 & 5 & 1 & 2 & 3 & 4 & 5 \\
\hline 39. & $\begin{array}{l}\text { Knowledge of the structure of the skeletal muscle fiber and } \\
\text { the basic mechanism of contraction }\end{array}$ & 1 & 2 & 3 & 4 & 5 & 1 & 2 & 3 & 4 & 5 \\
\hline 40. & Knowledge of the characteristics of fast and slow twitch fibers & 1 & 2 & 3 & 4 & 5 & 1 & 2 & 3 & 4 & 5 \\
\hline 41. & $\begin{array}{l}\text { Knowledge of the sliding filament theory of muscle } \\
\text { contraction }\end{array}$ & 1 & 2 & 3 & 4 & 5 & 1 & 2 & 3 & 4 & 5 \\
\hline 42. & $\begin{array}{l}\text { Knowledge of twitch, summation, and tetanus with respect to } \\
\text { muscle contraction }\end{array}$ & 1 & 2 & 3 & 4 & 5 & 1 & 2 & 3 & 4 & 5 \\
\hline 43. & $\begin{array}{l}\text { Ability to discuss the physiological principles involved in } \\
\text { promoting gains in muscular strength and endurance }\end{array}$ & 1 & 2 & 3 & 4 & 5 & 1 & 2 & 3 & 4 & 5 \\
\hline 44. & $\begin{array}{l}\text { Ability to define muscular fatigue as it relates to task, } \\
\text { intensity, duration, and the accumulative effects of exercise }\end{array}$ & 1 & 2 & 3 & 4 & 5 & 1 & 2 & 3 & 4 & 5 \\
\hline 45. & $\begin{array}{l}\text { Knowledge of the relationship between the number of } \\
\text { repetitions, intensity, number of sets, and rest with regard to } \\
\text { strength training }\end{array}$ & 1 & 2 & 3 & 4 & 5 & 1 & 2 & 3 & 4 & 5 \\
\hline
\end{tabular}




\begin{tabular}{|c|c|c|c|c|c|c|c|c|c|c|c|}
\hline & $\begin{array}{l}\text { Knowledge of the basic properties of cardiac muscle and the } \\
\text { normal pathways of conduction in the heart }\end{array}$ & 1 & 2 & 3 & 4 & 5 & 1 & 2 & 3 & 4 & 5 \\
\hline 47. & $\begin{array}{l}\text { Knowledge of the response of the following variables to acute } \\
\text { exercise: heart rate, stroke volume, cardiac output, pulmonary } \\
\text { ventilation, tidal volume, respiratory rate, and arteriovenous } \\
\text { oxygen difference }\end{array}$ & 1 & 2 & 3 & 4 & 5 & 1 & 2 & 3 & 4 & 5 \\
\hline 48. & $\begin{array}{l}\text { Knowledge of the differences in the cardiorespiratory } \\
\text { responses to static exercise compared with dynamic exercise, } \\
\text { including possible hazards and contraindications }\end{array}$ & 1 & 2 & 3 & 4 & 5 & 1 & 2 & 3 & 4 & 5 \\
\hline 49. & $\begin{array}{l}\text { Ability to describe how each of the following differs from the } \\
\text { normal condition: premature atrial contractions and premature } \\
\text { ventricular contractions }\end{array}$ & 1 & 2 & 3 & 4 & 5 & 1 & 2 & 3 & 4 & 5 \\
\hline 50. & $\begin{array}{l}\text { Knowledge of blood pressure responses associated with acute } \\
\text { exercise, including changes in body position }\end{array}$ & 1 & 2 & 3 & 4 & 5 & 1 & 2 & 3 & 4 & 5 \\
\hline 51. & $\begin{array}{l}\text { Knowledge of and ability to describe the implications of } \\
\text { ventilatory threshold (anaerobic threshold) as it relates to } \\
\text { exercise training and cardiorespiratory assessment }\end{array}$ & 1 & 2 & 3 & 4 & 5 & 1 & 2 & 3 & 4 & 5 \\
\hline 52. & $\begin{array}{l}\text { Knowledge of and ability to describe the physiological } \\
\text { adaptations of the respiratory system that occur at rest and } \\
\text { during submaximal and maximal exercise following chronic } \\
\text { aerobic and anaerobic training }\end{array}$ & 1 & 2 & 3 & 4 & 5 & 1 & 2 & 3 & 4 & 5 \\
\hline 53. & $\begin{array}{l}\text { Ability to describe how each of the following differs from the } \\
\text { normal condition: dyspnea, hypoxia, and hypoventilation }\end{array}$ & 1 & 2 & 3 & 4 & 5 & 1 & 2 & 3 & 4 & 5 \\
\hline 54. & $\begin{array}{l}\text { Knowledge of and ability to discuss the physiological basis of } \\
\text { the major components of physical fitness: flexibility, } \\
\text { cardiovascular fitness, muscular strength, muscular } \\
\text { endurance, and body composition }\end{array}$ & 1 & 2 & 3 & 4 & 5 & 1 & 2 & 3 & 4 & 5 \\
\hline 55. & $\begin{array}{l}\text { Ability to explain how the principle of specificity relates to } \\
\text { the components of fitness }\end{array}$ & 1 & 2 & 3 & 4 & 5 & 1 & 2 & 3 & 4 & 5 \\
\hline 56. & $\begin{array}{l}\text { Ability to explain the concept of detraining or reversibility of } \\
\text { conditioning and its implications in fitness programs }\end{array}$ & 1 & 2 & 3 & 4 & 5 & 1 & 2 & 3 & 4 & 5 \\
\hline 57. & $\begin{array}{l}\text { Ability to discuss the physical and psychological signs of } \\
\text { overtraining and provide recommendations for these problems }\end{array}$ & 1 & 2 & 3 & 4 & 5 & 1 & 2 & 3 & 4 & 5 \\
\hline 58. & $\begin{array}{l}\text { Ability to describe the physiological and metabolic responses } \\
\text { to exercise associated with chronic disease (heart disease, } \\
\text { hypertension, diabetes mellitus, and pulmonary disease) }\end{array}$ & 1 & 2 & 3 & 4 & 5 & 1 & 2 & 3 & 4 & 5 \\
\hline
\end{tabular}




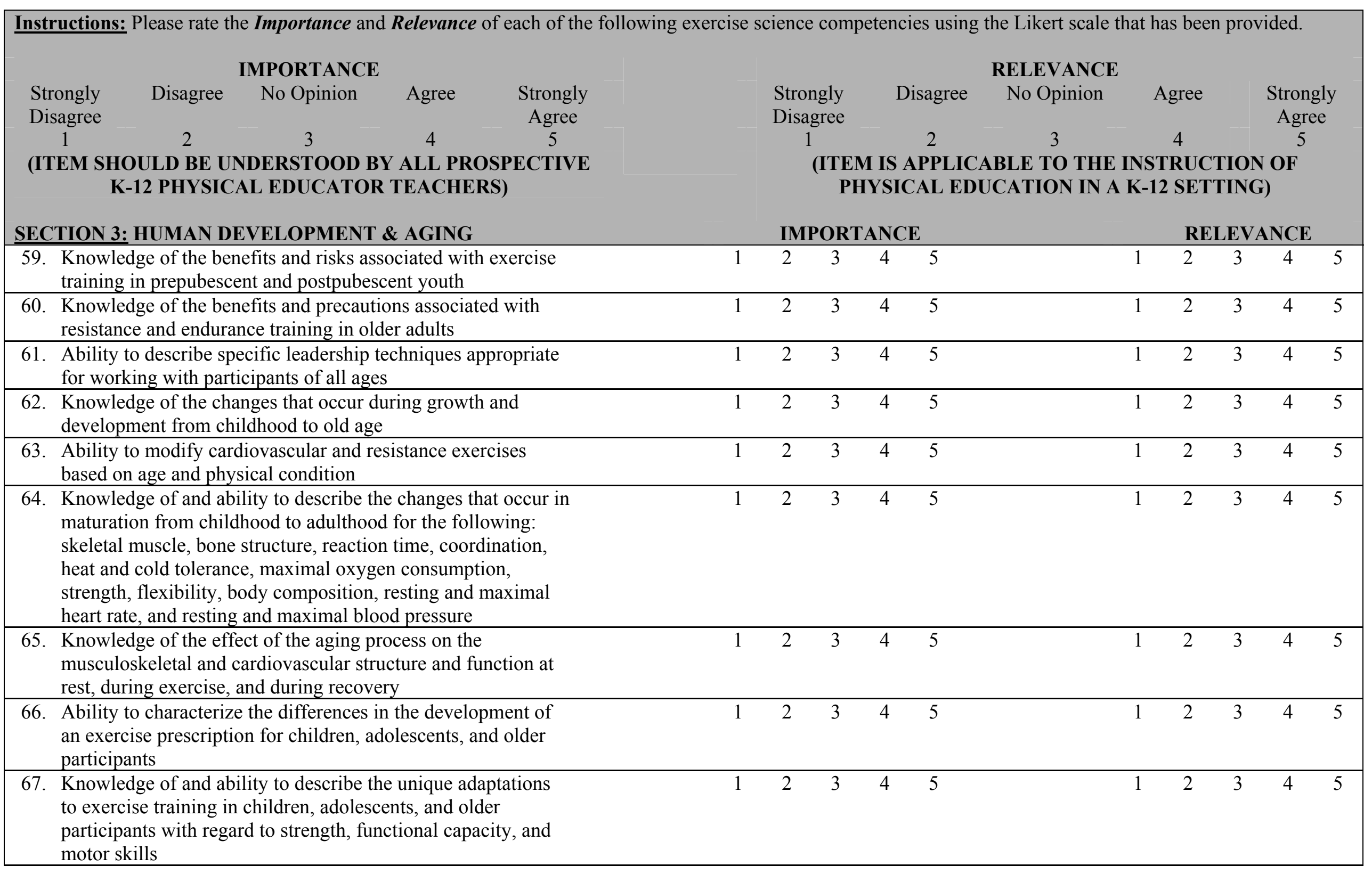


68. Knowledge of the common orthopedic and cardiovascular considerations for older participants and the ability to describe modifications in exercise prescription that are indicated

69. Knowledge of the health-related benefits of regular physical activity for people of all ages

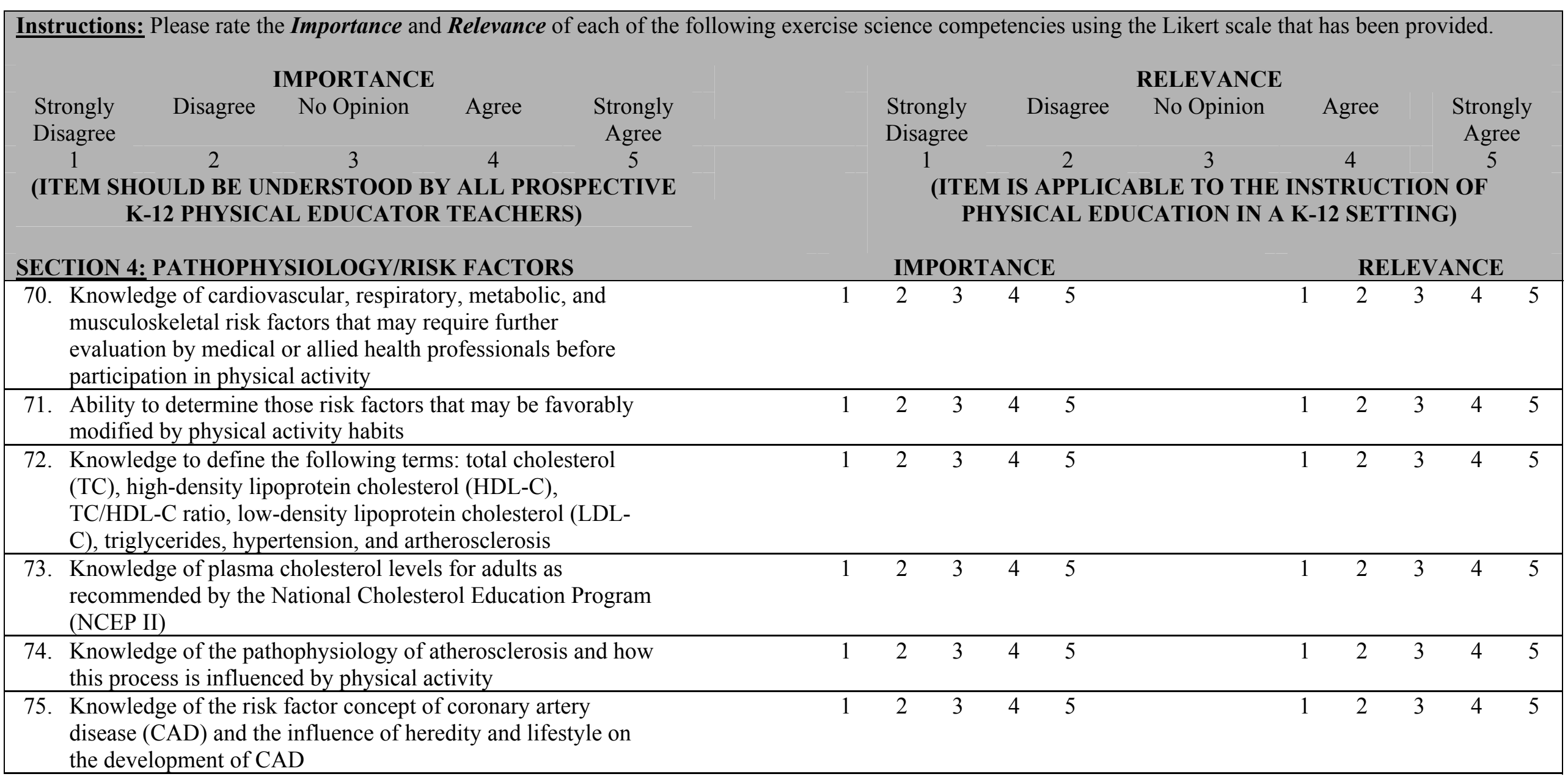




\begin{tabular}{|c|c|c|c|c|c|c|c|c|c|c|c|}
\hline & $\begin{array}{l}\text { Knowledge of the artherosclerotic process, the factors } \\
\text { involved in its genesis and progression, and the potential role } \\
\text { of exercise training in treatment }\end{array}$ & 1 & 2 & 3 & 4 & 5 & 1 & 2 & 3 & 4 & 5 \\
\hline 77. & $\begin{array}{l}\text { Ability to discuss in detail how lifestyle factors, including } \\
\text { nutrition, physical activity, and heredity, influence lipid and } \\
\text { lipoprotein profiles }\end{array}$ & 1 & 2 & 3 & 4 & 5 & 1 & 2 & 3 & 4 & 5 \\
\hline 78. & $\begin{array}{l}\text { Knowledge of cardiovascular risk factors or conditions that } \\
\text { may require consultation with medical personnel before } \\
\text { testing or training, including inappropriate changes in resting } \\
\text { or exercise heart rate and blood pressure, new onset } \\
\text { discomfort in chest, neck, shoulder, or arm, changes in the } \\
\text { pattern of discomfort during rest or exercise, fainting or dizzy } \\
\text { spells, and claudication }\end{array}$ & 1 & 2 & 3 & 4 & 5 & 1 & 2 & 3 & 4 & 5 \\
\hline 79. & $\begin{array}{l}\text { Knowledge of the respiratory risk factors or conditions that } \\
\text { may require consultation with medical personnel before } \\
\text { testing or training, including asthma, exercise-induced } \\
\text { bronchospasm, extreme breathlessness at rest or during } \\
\text { exercise, bronchitis, and emphysema }\end{array}$ & 1 & 2 & 3 & 4 & 5 & 1 & 2 & 3 & 4 & 5 \\
\hline 80. & $\begin{array}{l}\text { Knowledge of the metabolic risk factors or conditions that } \\
\text { may require consultation with medical personnel before } \\
\text { testing or training, including bodyweight more than } 20 \% \\
\text { above optimal, BMI }>30 \text {, thyroid disease, diabetes or glucose } \\
\text { intolerance, and hypoglycemia }\end{array}$ & 1 & 2 & 3 & 4 & 5 & 1 & 2 & 3 & 4 & 5 \\
\hline 81. & $\begin{array}{l}\text { Knowledge of the musculoskeletal risk factors or conditions } \\
\text { that may require consultation with medical personnel before } \\
\text { testing or training, including acute or chronic back pain, } \\
\text { osteoarthritis, rheumatoid arthritis, osteoporosis, tendonitis, } \\
\text { and low back pain }\end{array}$ & 1 & 2 & 3 & 4 & 5 & 1 & 2 & 3 & 4 & 5 \\
\hline 82. & $\begin{array}{l}\text { Knowledge of persons with physical and mental disabilities } \\
\text { and the potential influence on risk for chronic degenerative } \\
\text { disease }\end{array}$ & 1 & 2 & 3 & 4 & 5 & 1 & 2 & 3 & 4 & 5 \\
\hline 83. & $\begin{array}{l}\text { Knowledge of the factors that increase risk for osteoporosis } \\
\text { and the preventive measures that can be followed to decrease } \\
\text { risk }\end{array}$ & 1 & 2 & 3 & 4 & 5 & 1 & 2 & 3 & 4 & 5 \\
\hline
\end{tabular}




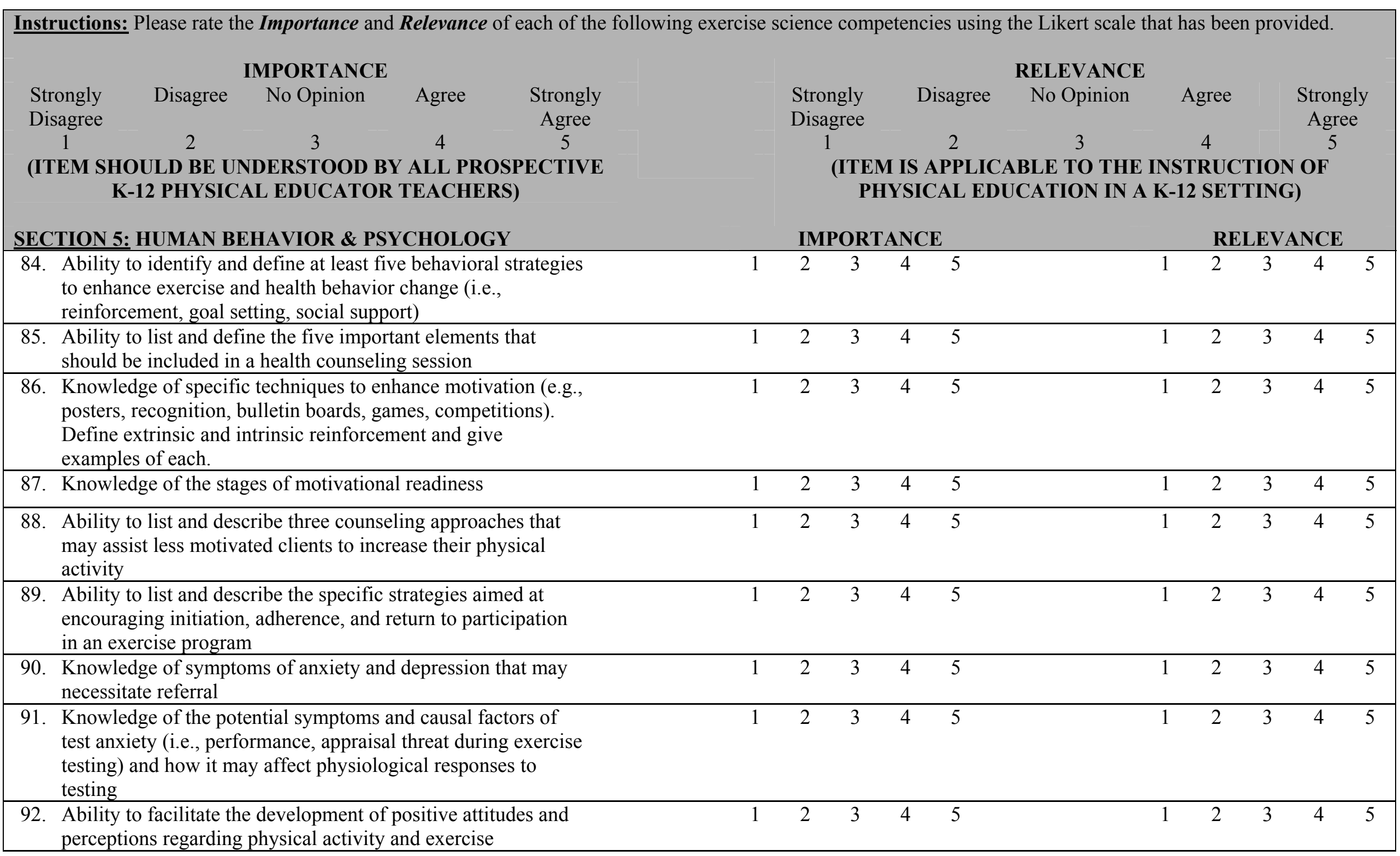




\begin{tabular}{|c|c|c|c|c|c|c|c|c|c|c|c|}
\hline & $\begin{array}{l}\text { Knowledge of the use of goal setting and behavioral contracts } \\
\text { as key components of the behavior change process }\end{array}$ & 1 & 2 & 3 & 4 & 5 & 1 & 2 & 3 & 4 & 5 \\
\hline 94. & $\begin{array}{l}\text { Knowledge of the psycho-social determinants of physical } \\
\text { activity (e.g., parental/peer influence, self-efficacy, self- } \\
\text { perception) }\end{array}$ & 1 & 2 & 3 & 4 & 5 & 1 & 2 & 3 & 4 & 5 \\
\hline 95. & $\begin{array}{l}\text { Knowledge of the environmental determinants of physical } \\
\text { activity (e.g., television/computers, access to exercise } \\
\text { facilities, neighborhood safety) }\end{array}$ & 1 & 2 & 3 & 4 & 5 & 1 & 2 & 3 & 4 & 5 \\
\hline 96. & $\begin{array}{l}\text { Knowledge of basic behavioral principles as related to } \\
\text { classroom management and establishment of a safe, exercise } \\
\text { environment }\end{array}$ & 1 & 2 & 3 & 4 & 5 & 1 & 2 & 3 & 4 & 5 \\
\hline 97. & $\begin{array}{l}\text { Knowledge of various educational theories and instructional } \\
\text { methods that can be used in the area of physical activity } \\
\text { promotion }\end{array}$ & 1 & 2 & 3 & 4 & 5 & 1 & 2 & 3 & 4 & 5 \\
\hline 98. & $\begin{array}{l}\text { Knowledge of the psychological benefits associated with } \\
\text { participation in regular physical activity }\end{array}$ & 1 & 2 & 3 & 4 & 5 & 1 & 2 & 3 & 4 & 5 \\
\hline 99. & $\begin{array}{l}\text { Knowledge of the psychological variables associated with } \\
\text { sport performance (e.g., anxiety, motivation, burnout) }\end{array}$ & 1 & 2 & 3 & 4 & 5 & 1 & 2 & 3 & 4 & 5 \\
\hline & $\begin{array}{l}\text { Knowledge of the signs/symptoms of disordered eating and } \\
\text { the appropriate procedures for referral }\end{array}$ & 1 & 2 & 3 & 4 & 5 & 1 & 2 & 3 & 4 & 5 \\
\hline
\end{tabular}
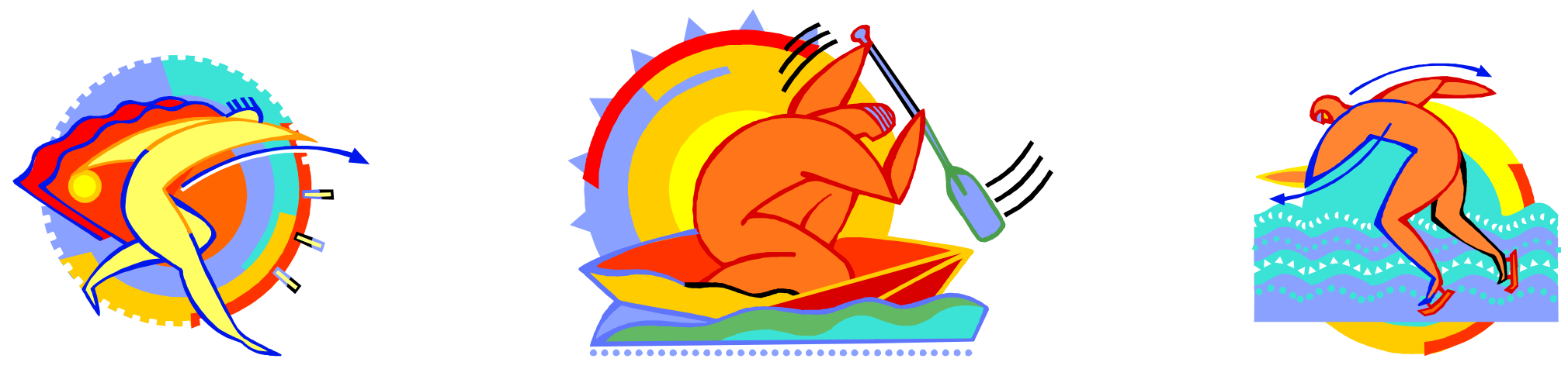

\section{Please continue on the next page...}




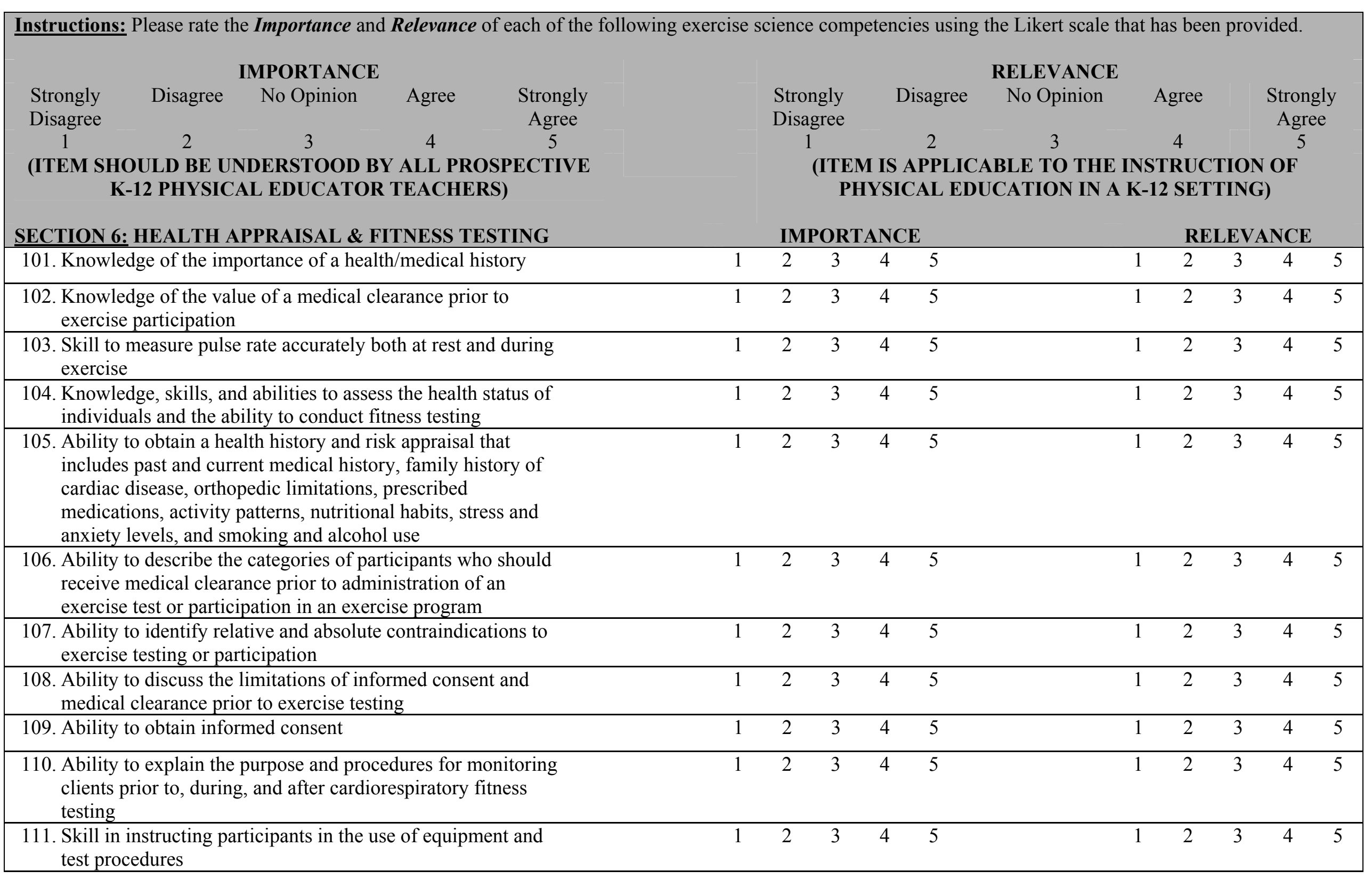




\begin{tabular}{|c|c|c|c|c|c|c|c|c|c|c|}
\hline $\begin{array}{l}\text { 112. Ability to describe the purpose of testing, select an } \\
\text { appropriate submaximal or maximal protocol, and conduct an } \\
\text { assessment of cardiovascular fitness on the cycle ergometer or } \\
\text { the treadmill }\end{array}$ & 1 & 2 & 3 & 4 & 5 & 1 & 2 & 3 & 4 & 5 \\
\hline $\begin{array}{l}\text { 113. Skill in accurately measuring heart rate, blood pressure, and } \\
\text { obtaining rating of perceived exertion (RPE) at rest and } \\
\text { during exercise according to established guidelines }\end{array}$ & 1 & 2 & 3 & 4 & 5 & 1 & 2 & 3 & 4 & 5 \\
\hline $\begin{array}{l}\text { 114. Ability to locate and measure skinfold sites, skeletal } \\
\text { diameters, and girth measurements used for estimating body } \\
\text { composition }\end{array}$ & 1 & 2 & 3 & 4 & 5 & 1 & 2 & 3 & 4 & 5 \\
\hline $\begin{array}{l}\text { 115. Ability to describe the purpose of testing, select appropriate } \\
\text { protocols, and conduct assessments of muscular strength, } \\
\text { muscular endurance, and flexibility }\end{array}$ & 1 & 2 & 3 & 4 & 5 & 1 & 2 & 3 & 4 & 5 \\
\hline 116. Skill in various techniques of assessing body composition & 1 & 2 & 3 & 4 & 5 & 1 & 2 & 3 & 4 & 5 \\
\hline $\begin{array}{l}\text { 117. Knowledge of the advantages/disadvantages and limitations } \\
\text { of the various body composition techniques }\end{array}$ & 1 & 2 & 3 & 4 & 5 & 1 & 2 & 3 & 4 & 5 \\
\hline $\begin{array}{l}\text { 118. Ability to interpret information obtained from } \\
\text { cardiorespiratory fitness tests and muscular } \\
\text { strength/endurance, flexibility, and body composition tests for } \\
\text { apparently healthy individuals and those with stable disease }\end{array}$ & 1 & 2 & 3 & 4 & 5 & 1 & 2 & 3 & 4 & 5 \\
\hline $\begin{array}{l}\text { 119. Ability to identify appropriate criteria for terminating a fitness } \\
\text { evaluation and demonstrate proper procedures to be followed } \\
\text { after discontinuing such a test }\end{array}$ & 1 & 2 & 3 & 4 & 5 & 1 & 2 & 3 & 4 & 5 \\
\hline $\begin{array}{l}\text { 120. Ability to modify protocols and procedures for } \\
\text { cardiorespiratory fitness tests in children, adolescents, and } \\
\text { older adults }\end{array}$ & 1 & 2 & 3 & 4 & 5 & 1 & 2 & 3 & 4 & 5 \\
\hline $\begin{array}{l}\text { 121. Ability to conduct fitness appraisals for populations with } \\
\text { physical and mental disabilities (e.g., mental retardation) } \\
\text { which may require alternative testing techniques }\end{array}$ & 1 & 2 & 3 & 4 & 5 & 1 & 2 & 3 & 4 & 5 \\
\hline $\begin{array}{l}\text { 122. Knowledge of the steps needed to prepare children for } \\
\text { physical fitness testing (e.g., teaching test purpose, protocol, } \\
\text { pacing) }\end{array}$ & 1 & 2 & 3 & 4 & 5 & 1 & 2 & 3 & 4 & 5 \\
\hline $\begin{array}{l}\text { 123. Knowledge of the differences between norm and criterion } \\
\text { referenced fitness tests }\end{array}$ & 1 & 2 & 3 & 4 & 5 & 1 & 2 & 3 & 4 & 5 \\
\hline
\end{tabular}




\begin{tabular}{|c|c|c|c|c|c|c|c|c|c|c|}
\hline $\begin{array}{l}\text { 124. Knowledge of the role of physical fitness testing in achieving } \\
\text { national and state standards for physical education }\end{array}$ & 1 & 2 & 3 & 4 & 5 & 1 & 2 & 3 & 4 & 5 \\
\hline $\begin{array}{l}\text { 125. Ability to use heart rate monitors for monitoring intensity } \\
\text { during exercise }\end{array}$ & 1 & 2 & 3 & 4 & 5 & 1 & 2 & 3 & 4 & 5 \\
\hline $\begin{array}{l}\text { 126. Knowledge of the NIH definitions of obesity and overweight } \\
\text { using body mass index (BMI) }\end{array}$ & 1 & 2 & 3 & 4 & 5 & 1 & 2 & 3 & 4 & 5 \\
\hline $\begin{array}{l}\text { 127. Ability to use pedometers for monitoring physical activity } \\
\text { participation }\end{array}$ & 1 & 2 & 3 & 4 & 5 & 1 & 2 & 3 & 4 & 5 \\
\hline \multicolumn{11}{|c|}{$\begin{array}{l}\text { Knowledge of common drugs from each of the following classes of medications and describe the principal action and the effects on exercise testing and } \\
\text { prescription: }\end{array}$} \\
\hline 128. Bronchodilators & 1 & 2 & 3 & 4 & 5 & 1 & 2 & 3 & 4 & 5 \\
\hline 129. Hypoglycemics & 1 & 2 & 3 & 4 & 5 & 1 & 2 & 3 & 4 & 5 \\
\hline 130. Psychotropics & 1 & 2 & 3 & 4 & 5 & 1 & 2 & 3 & 4 & 5 \\
\hline 131. Vasodilators & 1 & 2 & 3 & 4 & 5 & 1 & 2 & 3 & 4 & 5 \\
\hline $\begin{array}{l}\text { 132. Ability to identify the effects of the following substances on } \\
\text { exercise response: antihistamines, tranquilizers, alcohol, diet } \\
\text { pills, cold tablets, caffeine, and nicotine }\end{array}$ & 1 & 2 & 3 & 4 & 5 & 1 & 2 & 3 & 4 & 5 \\
\hline
\end{tabular}

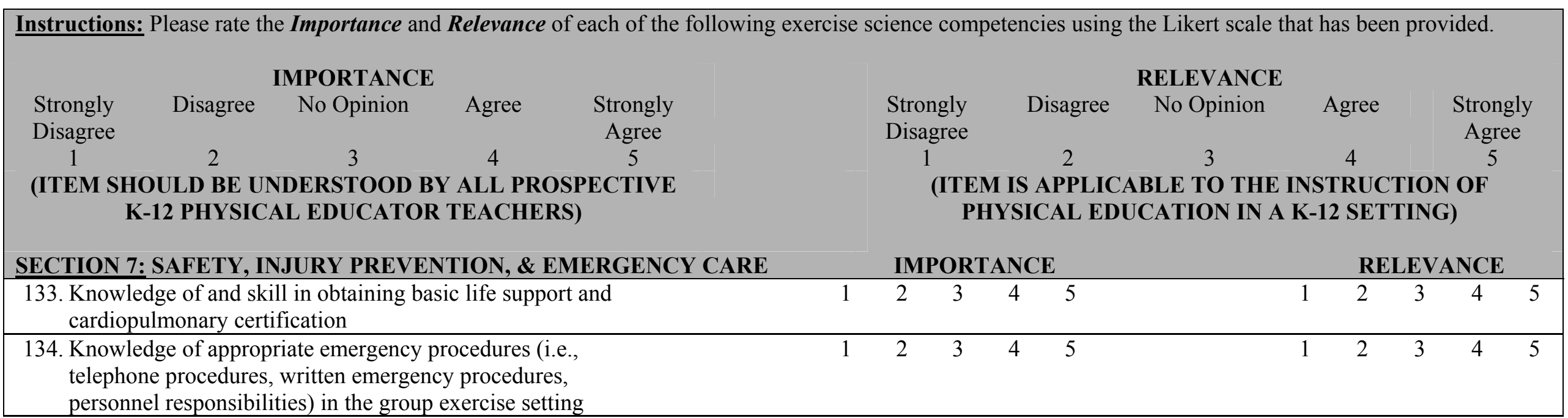




\begin{tabular}{|c|c|c|c|c|c|c|c|c|c|c|}
\hline $\begin{array}{l}\text { 135. Knowledge of basic first aid procedures for exercise-related } \\
\text { injuries, such as bleeding, strains/sprains, fractures, and } \\
\text { exercise intolerance (dizziness, syncope, heat injury) }\end{array}$ & 1 & 2 & 3 & 4 & 5 & 1 & 2 & 3 & 4 & 5 \\
\hline $\begin{array}{l}\text { 136. Knowledge of basic precautions taken in a group exercise } \\
\text { setting to ensure participant safety }\end{array}$ & 1 & 2 & 3 & 4 & 5 & 1 & 2 & 3 & 4 & 5 \\
\hline $\begin{array}{l}\text { 137. Ability to identify the physical and physiological signs and } \\
\text { symptoms of overtraining }\end{array}$ & 1 & 2 & 3 & 4 & 5 & 1 & 2 & 3 & 4 & 5 \\
\hline $\begin{array}{l}\text { 138. Ability to list the effects of temperature, humidity, altitude, } \\
\text { and pollution on physiological response to exercise }\end{array}$ & 1 & 2 & 3 & 4 & 5 & 1 & 2 & 3 & 4 & 5 \\
\hline $\begin{array}{l}\text { 139. Knowledge of the following terms: shin splints, sprain, strain, } \\
\text { tennis elbow, bursitis, stress fracture, tendonitis, patellar } \\
\text { femoral pain syndrome, low back pain, plantar fasciitis, and } \\
\text { rotator cuff tendonitis }\end{array}$ & 1 & 2 & 3 & 4 & 5 & 1 & 2 & 3 & 4 & 5 \\
\hline $\begin{array}{l}\text { 140. Skill to demonstrate exercises used for people with low back } \\
\text { pain }\end{array}$ & 1 & 2 & 3 & 4 & 5 & 1 & 2 & 3 & 4 & 5 \\
\hline $\begin{array}{l}\text { 141. Knowledge of hypothetical concerns and potential risks that } \\
\text { may be associated with the use of exercises such as straight } \\
\text { leg sit-ups, double leg raises, full squats, hurdlers stretch, } \\
\text { yoga plough, forceful back hyperextension, and standing bent- } \\
\text { over toe touch }\end{array}$ & 1 & 2 & 3 & 4 & 5 & 1 & 2 & 3 & 4 & 5 \\
\hline $\begin{array}{l}\text { 142. Skill in demonstrating appropriate emergency procedures } \\
\text { during exercise testing and/or training }\end{array}$ & 1 & 2 & 3 & 4 & 5 & 1 & 2 & 3 & 4 & 5 \\
\hline $\begin{array}{l}\text { 143. Knowledge of safety plans, emergency procedures, and first } \\
\text { aid techniques needed during fitness evaluations, exercise } \\
\text { testing, and exercise training }\end{array}$ & 1 & 2 & 3 & 4 & 5 & 1 & 2 & 3 & 4 & 5 \\
\hline $\begin{array}{l}\text { 144. Ability to identify the components that contribute to the } \\
\text { maintenance of a safe environment }\end{array}$ & 1 & 2 & 3 & 4 & 5 & 1 & 2 & 3 & 4 & 5 \\
\hline $\begin{array}{l}\text { 145. Knowledge of the health/fitness instructor's responsibilities, } \\
\text { limitations, and the legal implications of carrying out } \\
\text { emergency procedures }\end{array}$ & 1 & 2 & 3 & 4 & 5 & 1 & 2 & 3 & 4 & 5 \\
\hline $\begin{array}{l}\text { 146. Ability to describe potential musculoskeletal injuries (e.g., } \\
\text { contusions, sprains, strains, fractures), } \\
\text { cardiovascular/pulmonary complications (e.g., tachycardia, } \\
\text { bradycardia, hypotension/hypertension, tachypnea), and } \\
\text { metabolic abnormalities (e.g., fainting/syncope, } \\
\text { hypoglycemia/hyperglycemia, hypothermia/hyperthermia) }\end{array}$ & 1 & 2 & 3 & 4 & 5 & 1 & 2 & 3 & 4 & 5 \\
\hline
\end{tabular}


147. Knowledge of the initial management and first aid techniques associated with open wounds, musculoskeletal injuries, cardiovascular/pulmonary complications, and metabolic disorders

148. Knowledge of the components of an equipment maintenance/repair program and how it may be used to evaluate the condition of exercise equipment to reduce potential risk of injury

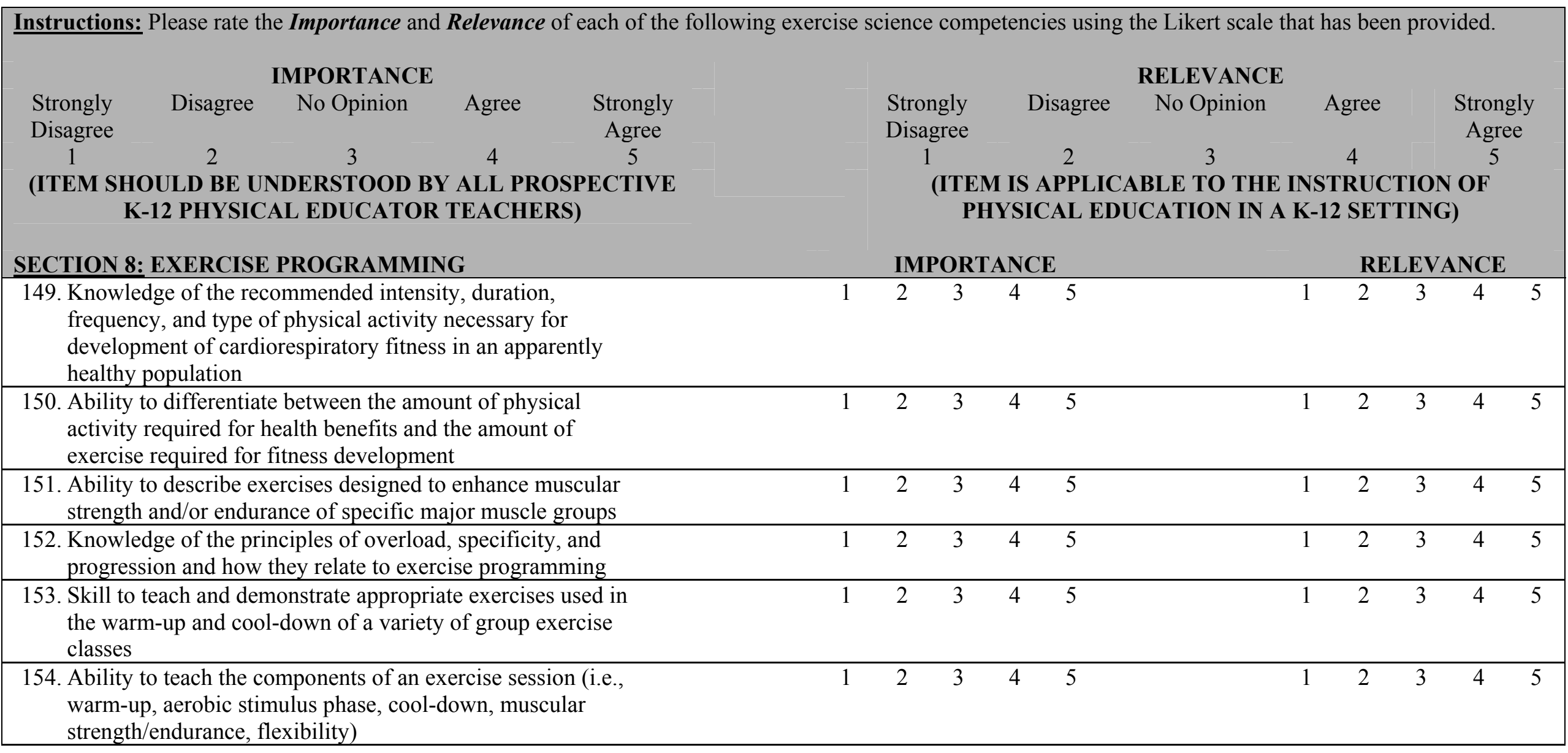




\begin{tabular}{|c|c|c|c|c|c|c|c|c|c|c|}
\hline $\begin{array}{l}\text { 155. Knowledge of the following terms: progressive resistance, } \\
\text { isotonic/isometric, concentric, eccentric, atrophy, } \\
\text { hypertrophy, sets, repetitions, plyometrics, Valsalva maneuver }\end{array}$ & 1 & 2 & 3 & 4 & 5 & 1 & 2 & 3 & 4 & 5 \\
\hline $\begin{array}{l}\text { 156. Skill to teach class participants how to monitor intensity of } \\
\text { exercise using heart rate and rating of perceived exertion } \\
\text { (RPE) }\end{array}$ & 1 & 2 & 3 & 4 & 5 & 1 & 2 & 3 & 4 & 5 \\
\hline $\begin{array}{l}\text { 157. Skill to teach participants how to use RPE and heart rate to } \\
\text { adjust the intensity of the exercise session }\end{array}$ & 1 & 2 & 3 & 4 & 5 & 1 & 2 & 3 & 4 & 5 \\
\hline $\begin{array}{l}\text { 158. Ability to calculate training heart rates using two methods: } \\
\text { percent of age-predicted maximum heart rate and heart rate } \\
\text { reserve (Karvonen) }\end{array}$ & 1 & 2 & 3 & 4 & 5 & 1 & 2 & 3 & 4 & 5 \\
\hline $\begin{array}{l}\text { 159. Skill to teach and demonstrate appropriate modifications in } \\
\text { specific exercises for the following groups: older adults, } \\
\text { pregnant and postnatal women, obese persons, and persons } \\
\text { with low back pain }\end{array}$ & 1 & 2 & 3 & 4 & 5 & 1 & 2 & 3 & 4 & 5 \\
\hline $\begin{array}{l}\text { 160. Ability to recognize proper and improper techniques in the } \\
\text { use of resistive equipment such a stability balls, weights, } \\
\text { bands, resistance bars, and water exercise equipment }\end{array}$ & 1 & 2 & 3 & 4 & 5 & 1 & 2 & 3 & 4 & 5 \\
\hline $\begin{array}{l}\text { 161. Ability to recognize proper and improper technique in the use } \\
\text { of cardiovascular conditioning equipment (e.g., steps, cycles, } \\
\text { slides) }\end{array}$ & 1 & 2 & 3 & 4 & 5 & 1 & 2 & 3 & 4 & 5 \\
\hline $\begin{array}{l}\text { 162. Skill to teach and demonstrate appropriate exercises for } \\
\text { improving range of motion of all major joints }\end{array}$ & 1 & 2 & 3 & 4 & 5 & 1 & 2 & 3 & 4 & 5 \\
\hline $\begin{array}{l}\text { 163. Ability to modify exercises in the group setting for apparently } \\
\text { healthy persons of various fitness levels }\end{array}$ & 1 & 2 & 3 & 4 & 5 & 1 & 2 & 3 & 4 & 5 \\
\hline $\begin{array}{l}\text { 164. Ability to teach a progression of exercises for all major } \\
\text { muscle groups to improve muscular strength and endurance }\end{array}$ & 1 & 2 & 3 & 4 & 5 & 1 & 2 & 3 & 4 & 5 \\
\hline $\begin{array}{l}\text { 165. Knowledge to describe the various types of interval, } \\
\text { continuous, and circuit training programs }\end{array}$ & 1 & 2 & 3 & 4 & 5 & 1 & 2 & 3 & 4 & 5 \\
\hline $\begin{array}{l}\text { 166. Knowledge to describe various ways a leader can take a } \\
\text { position relative to the group to enhance visibility, participant } \\
\text { interactions, and communication }\end{array}$ & 1 & 2 & 3 & 4 & 5 & 1 & 2 & 3 & 4 & 5 \\
\hline $\begin{array}{l}\text { 167. Ability to communicate effectively with exercise participants } \\
\text { in the group exercise session }\end{array}$ & 1 & 2 & 3 & 4 & 5 & 1 & 2 & 3 & 4 & 5 \\
\hline $\begin{array}{l}\text { 168. Knowledge to describe partner resistance exercises that can be } \\
\text { used in a group class setting }\end{array}$ & 1 & 2 & 3 & 4 & 5 & 1 & 2 & 3 & 4 & 5 \\
\hline
\end{tabular}




\begin{tabular}{|c|c|c|c|c|c|c|c|c|c|c|}
\hline $\begin{array}{l}\text { 169. Ability to demonstrate techniques for accommodating various } \\
\text { fitness levels within the same class }\end{array}$ & 1 & 2 & 3 & 4 & 5 & 1 & 2 & 3 & 4 & 5 \\
\hline $\begin{array}{l}\text { 170. Knowledge of the properties of water that affect the design of } \\
\text { a water exercise session }\end{array}$ & 1 & 2 & 3 & 4 & 5 & 1 & 2 & 3 & 4 & 5 \\
\hline $\begin{array}{l}\text { 171. Knowledge of basic music fundamentals, including downbeat, } \\
8 \text { count, and } 32 \text { count }\end{array}$ & 1 & 2 & 3 & 4 & 5 & 1 & 2 & 3 & 4 & 5 \\
\hline $\begin{array}{l}\text { 172. Skill to effectively use verbal and nonverbal cues in the group } \\
\text { exercise setting, including anticipatory, motivational, safety, } \\
\text { and educational }\end{array}$ & 1 & 2 & 3 & 4 & 5 & 1 & 2 & 3 & 4 & 5 \\
\hline $\begin{array}{l}\text { 173. Skill to demonstrate the proper form, alignment, and } \\
\text { technique in typical exercises used in warm-up, stimulus, } \\
\text { muscle conditioning, and cool-down phases of the group } \\
\text { session }\end{array}$ & 1 & 2 & 3 & 4 & 5 & 1 & 2 & 3 & 4 & 5 \\
\hline $\begin{array}{l}\text { 174. Ability to evaluate specific exercises in terms of safety and } \\
\text { effectiveness for various participants }\end{array}$ & 1 & 2 & 3 & 4 & 5 & 1 & 2 & 3 & 4 & 5 \\
\hline $\begin{array}{l}\text { 175. Ability to demonstrate a familiarity with a variety of group } \\
\text { exercise formats (e.g., traditional, step, slide, muscle } \\
\text { conditioning, flexibility, indoor cycling, water fitness, } \\
\text { walking) }\end{array}$ & 1 & 2 & 3 & 4 & 5 & 1 & 2 & 3 & 4 & 5 \\
\hline $\begin{array}{l}\text { 176. Knowledge, skills, and abilities to prescribe and administer } \\
\text { exercise programs for apparently healthy individuals, } \\
\text { individuals at higher risk, and individuals with known disease }\end{array}$ & 1 & 2 & 3 & 4 & 5 & 1 & 2 & 3 & 4 & 5 \\
\hline $\begin{array}{l}\text { 177. Ability to design, implement, and evaluate individualized and } \\
\text { group exercise programs based on health history and physical } \\
\text { fitness assessments }\end{array}$ & 1 & 2 & 3 & 4 & 5 & 1 & 2 & 3 & 4 & 5 \\
\hline $\begin{array}{l}\text { 178. Ability to modify exercises based on age and physical } \\
\text { condition }\end{array}$ & 1 & 2 & 3 & 4 & 5 & 1 & 2 & 3 & 4 & 5 \\
\hline $\begin{array}{l}\text { 179. Knowledge, skills, and abilities to calculate energy cost, VO2, } \\
\text { METs, and target heart rates and apply the information to an } \\
\text { exercise prescription }\end{array}$ & 1 & 2 & 3 & 4 & 5 & 1 & 2 & 3 & 4 & 5 \\
\hline $\begin{array}{l}\text { 180. Ability to convert weights from pounds (lb) to kilograms }(\mathrm{kg}) \\
\text { and speed from miles per hour (MPH) to meters per minute } \\
(\mathrm{m} \cdot \mathrm{min}-1)\end{array}$ & 1 & 2 & 3 & 4 & 5 & 1 & 2 & 3 & 4 & 5 \\
\hline $\begin{array}{l}\text { 181. Ability to convert METs to VO2 expressed as } \mathrm{mL} \cdot \mathrm{kg}-1 \cdot \mathrm{min}-1 \text {, } \\
\mathrm{L} \cdot \mathrm{min}-1 \text {, and/or } \mathrm{mL} \cdot \mathrm{kg} \text { FFW- } 1 \cdot \mathrm{min}-1\end{array}$ & 1 & 2 & 3 & 4 & 5 & 1 & 2 & 3 & 4 & 5 \\
\hline
\end{tabular}




\begin{tabular}{|c|c|c|c|c|c|c|c|c|c|c|}
\hline $\begin{array}{l}\text { 182. Ability to calculate the energy cost in METs and kilocalories } \\
\text { for given exercise intensities in stepping exercise, cycle } \\
\text { ergometry, and during horizontal and graded walking and } \\
\text { running }\end{array}$ & 1 & 2 & 3 & 4 & 5 & 1 & 2 & 3 & 4 & 5 \\
\hline $\begin{array}{l}\text { 183. Knowledge of the approximate METs for various sport, } \\
\text { recreational, and work tasks }\end{array}$ & 1 & 2 & 3 & 4 & 5 & 1 & 2 & 3 & 4 & 5 \\
\hline $\begin{array}{l}\text { 184. Ability to prescribe exercise intensity based on } \mathrm{VO} 2 \text { data for } \\
\text { different modes of exercise, including graded and horizontal } \\
\text { running and walking, cycling, and stepping exercise }\end{array}$ & 1 & 2 & 3 & 4 & 5 & 1 & 2 & 3 & 4 & 5 \\
\hline $\begin{array}{l}\text { 185. Ability to explain and implement exercise prescription } \\
\text { guidelines for apparently health clients, increased risk clients, } \\
\text { and clients with controlled disease }\end{array}$ & 1 & 2 & 3 & 4 & 5 & 1 & 2 & 3 & 4 & 5 \\
\hline $\begin{array}{l}\text { 186. Ability to adapt frequency, intensity, duration, mode, } \\
\text { progression, level of supervision, and monitoring techniques } \\
\text { in exercise programs for patients with controlled chronic } \\
\text { disease (heart disease, diabetes mellitus, obesity, } \\
\text { hypertension), musculoskeletal problems, pregnancy and/or } \\
\text { postpartum and exercise-induced asthma }\end{array}$ & 1 & 2 & 3 & 4 & 5 & 1 & 2 & 3 & 4 & 5 \\
\hline $\begin{array}{l}\text { 187. Ability to understand the components incorporated into an } \\
\text { exercise session and the proper sequence (i.e., preexercise } \\
\text { evaluation, warm-up, aerobic stimulus phase, cool-down, } \\
\text { muscular strength and/or endurance, and flexibility) }\end{array}$ & 1 & 2 & 3 & 4 & 5 & 1 & 2 & 3 & 4 & 5 \\
\hline $\begin{array}{l}\text { 188. Skill in the use of various methods for establishing and } \\
\text { monitoring levels of exercise intensity, including heart rate, } \\
\text { RPE, and METs }\end{array}$ & 1 & 2 & 3 & 4 & 5 & 1 & 2 & 3 & 4 & 5 \\
\hline $\begin{array}{l}\text { 189. Knowledge of special precautions and modifications of } \\
\text { exercise programming for participation at altitude, different } \\
\text { ambient temperatures, humidity, and environmental pollution }\end{array}$ & 1 & 2 & 3 & 4 & 5 & 1 & 2 & 3 & 4 & 5 \\
\hline $\begin{array}{l}\text { 190. Ability to design resistive exercise programs to increase or } \\
\text { maintain muscular strength and/or endurance }\end{array}$ & 1 & 2 & 3 & 4 & 5 & 1 & 2 & 3 & 4 & 5 \\
\hline $\begin{array}{l}\text { 191. Ability to evaluate flexibility and prescribe appropriate } \\
\text { flexibility exercises for all major muscle groups }\end{array}$ & 1 & 2 & 3 & 4 & 5 & 1 & 2 & 3 & 4 & 5 \\
\hline $\begin{array}{l}\text { 192. Knowledge of the importance of recording exercise sessions } \\
\text { and performing periodic evaluations to assess changes in } \\
\text { fitness status }\end{array}$ & 1 & 2 & 3 & 4 & 5 & 1 & 2 & 3 & 4 & 5 \\
\hline
\end{tabular}




\begin{tabular}{|c|c|c|c|c|c|c|c|c|c|c|}
\hline $\begin{array}{l}\text { 193. Knowledge of the advantages and disadvantages of } \\
\text { implementation of interval, continuous, and circuit training } \\
\text { programs }\end{array}$ & 1 & 2 & 3 & 4 & 5 & 1 & 2 & 3 & 4 & 5 \\
\hline $\begin{array}{l}\text { 194. Ability to design training programs using interval, continuous, } \\
\text { and circuit training programs }\end{array}$ & 1 & 2 & 3 & 4 & 5 & 1 & 2 & 3 & 4 & 5 \\
\hline $\begin{array}{l}\text { 195. Ability to discuss the advantages and disadvantages of various } \\
\text { commercial exercise equipment in developing } \\
\text { cardiorespiratory fitness, muscular strength, and muscular } \\
\text { endurance }\end{array}$ & 1 & 2 & 3 & 4 & 5 & 1 & 2 & 3 & 4 & 5 \\
\hline $\begin{array}{l}\text { 196. Knowledge of the types of exercise programs available in the } \\
\text { community and how these programs are appropriate for } \\
\text { various populations }\end{array}$ & 1 & 2 & 3 & 4 & 5 & 1 & 2 & 3 & 4 & 5 \\
\hline $\begin{array}{l}\text { 197. Ability to include participants with different individual needs } \\
\text { into a group exercise environment }\end{array}$ & 1 & 2 & 3 & 4 & 5 & 1 & 2 & 3 & 4 & 5 \\
\hline $\begin{array}{l}\text { 198. Knowledge of the concept of periodization as it relates to } \\
\text { strength and endurance exercise programming }\end{array}$ & 1 & 2 & 3 & 4 & 5 & 1 & 2 & 3 & 4 & 5 \\
\hline $\begin{array}{l}\text { 199. Knowledge of the special concerns and guidelines } \\
\text { recommended for children and adolescent strength training }\end{array}$ & 1 & 2 & 3 & 4 & 5 & 1 & 2 & 3 & 4 & 5 \\
\hline $\begin{array}{l}\text { 200. Knowledge of the published exercise guidelines and } \\
\text { recommendations for children, adolescents, adults and older } \\
\text { adults }\end{array}$ & 1 & 2 & 3 & 4 & 5 & 1 & 2 & 3 & 4 & 5 \\
\hline
\end{tabular}

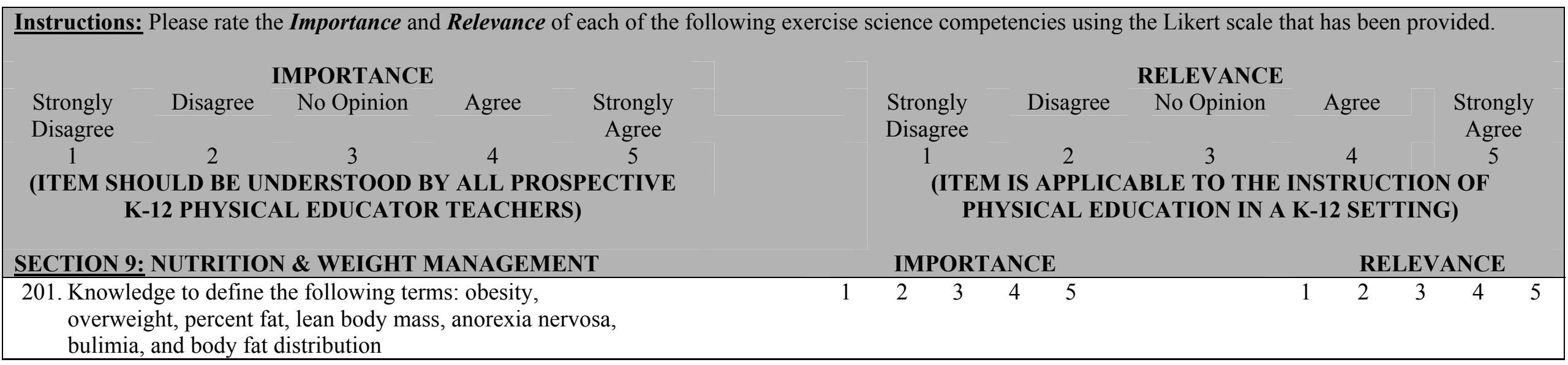




\begin{tabular}{|c|c|c|c|c|c|c|c|c|c|c|}
\hline $\begin{array}{l}\text { 202. Knowledge of the relationship between body composition and } \\
\text { health }\end{array}$ & 1 & 2 & 3 & 4 & 5 & 1 & 2 & 3 & 4 & 5 \\
\hline $\begin{array}{l}\text { 203. Knowledge of the effects of diet plus exercise, diet alone, and } \\
\text { exercise alone as methods for modifying body composition }\end{array}$ & 1 & 2 & 3 & 4 & 5 & 1 & 2 & 3 & 4 & 5 \\
\hline $\begin{array}{l}\text { 204. Knowledge of the importance of an adequate daily energy } \\
\text { intake for healthy weight management }\end{array}$ & 1 & 2 & 3 & 4 & 5 & 1 & 2 & 3 & 4 & 5 \\
\hline $\begin{array}{l}\text { 205. Ability to differentiate between fat soluble and water-soluble } \\
\text { vitamins }\end{array}$ & 1 & 2 & 3 & 4 & 5 & 1 & 2 & 3 & 4 & 5 \\
\hline $\begin{array}{l}\text { 206. Ability to describe the importance of maintaining normal } \\
\text { hydration before, during, and after exercise }\end{array}$ & 1 & 2 & 3 & 4 & 5 & 1 & 2 & 3 & 4 & 5 \\
\hline 207. Knowledge of the USDA Food Pyramid & 1 & 2 & 3 & 4 & 5 & 1 & 2 & 3 & 4 & 5 \\
\hline $\begin{array}{l}\text { 208. Knowledge of the importance of calcium and iron in women's } \\
\text { health }\end{array}$ & 1 & 2 & 3 & 4 & 5 & 1 & 2 & 3 & 4 & 5 \\
\hline $\begin{array}{l}\text { 209. Ability to describe the myths and consequences associated } \\
\text { with inappropriate weight loss methods (e.g., saunas, } \\
\text { vibrating belts, body wraps, electric simulators, sweat suits, } \\
\text { fad diets) }\end{array}$ & 1 & 2 & 3 & 4 & 5 & 1 & 2 & 3 & 4 & 5 \\
\hline $\begin{array}{l}\text { 210. Knowledge of the number of kilocalories in one gram of } \\
\text { carbohydrate, fat, protein, and alcohol }\end{array}$ & 1 & 2 & 3 & 4 & 5 & 1 & 2 & 3 & 4 & 5 \\
\hline $\begin{array}{l}\text { 211. Knowledge of the number of kilocalories equivalent to losing } \\
1 \text { pound of body fat }\end{array}$ & 1 & 2 & 3 & 4 & 5 & 1 & 2 & 3 & 4 & 5 \\
\hline $\begin{array}{l}\text { 212. Knowledge, skills, and abilities to provide information } \\
\text { concerning nutrition and the role of diet and exercise on body } \\
\text { composition and weight control }\end{array}$ & 1 & 2 & 3 & 4 & 5 & 1 & 2 & 3 & 4 & 5 \\
\hline $\begin{array}{l}\text { 213. Ability to describe the health implications of variation in body } \\
\text { fat distribution patterns and the significance of waist to hip } \\
\text { ratio }\end{array}$ & 1 & 2 & 3 & 4 & 5 & 1 & 2 & 3 & 4 & 5 \\
\hline $\begin{array}{l}\text { 214. Knowledge of the guidelines for caloric intake for an } \\
\text { individual desiring to lose or gain weight }\end{array}$ & 1 & 2 & 3 & 4 & 5 & 1 & 2 & 3 & 4 & 5 \\
\hline $\begin{array}{l}\text { 215. Knowledge of common nutritional ergogenic aids, the } \\
\text { purported mechanism of action, and any risk and/or benefit } \\
\text { (e.g., carbohydrates, protein/amino acids, vitamins, minerals, } \\
\text { sodium bicarbonate, creatine, bee pollen) }\end{array}$ & 1 & 2 & 3 & 4 & 5 & 1 & 2 & 3 & 4 & 5 \\
\hline
\end{tabular}


216. Knowledge of nutritional factors related to the female athlete triad syndrome (i.e., eating disorders, menstrual cycle abnormalities, and osteoporosis)

217. Knowledge of the NIH Consensus statement regarding health risks of obesity, Nutrition for Physical Fitness Position Paper of the American Dietetic Association, and the ACSM Position Stand on proper and improper weight loss programs

218. Knowledge of NCEP II guidelines for lipid management

2

3

45

5

$1 \quad 2 \quad 3$

$\begin{array}{ll}4 & 5\end{array}$

$\begin{array}{llll}2 & 3 & 4 & 5\end{array}$

$\begin{array}{llll}2 & 3 & 4 & 5\end{array}$

Instructions: Please rate the Importance and Relevance of each of the following exercise science competencies using the Likert scale that has been provided.

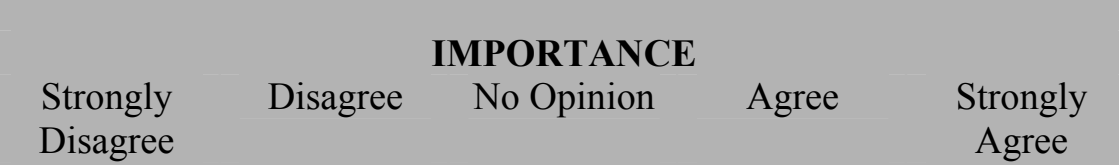

Disagree

1

2

4

K-12 PHYSICAL EDUCATOR TEACHERS)

(ITEM SHOULD BE UNDERSTOOD BY ALL PROSPECTIVE

\begin{tabular}{|lllllll|}
\hline 4 & 5 & 1 & 2 & 3 & 4 & 5
\end{tabular}

SECTION 10: PROGRAM \& ADMINISTRATION/MANAGEMENT

219. Knowledge, skills, and ability to administer and deliver health/fitness programs

220. Ability to describe and use the documentation required when a client shows signs or symptoms during an exercise session and should be referred to a physician

221. Ability to create and maintain records pertaining to participant exercise adherence, retention, and goal setting

222. Ability to develop and administer educational programs (e.g., lectures, workshops) and educational materials

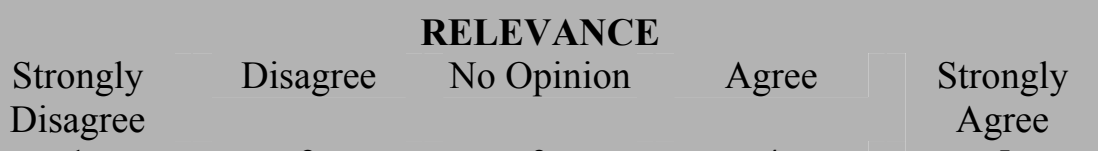
1
23
34
45
(ITEM IS APPLICABLE TO THE INSTRUCTION OF PHYSICAL EDUCATION IN A K-12 SETTING)

\section{THANKS AGAIN FOR YOUR TIME \& CONSIDERATION!}




\section{APPENDIX C}

Modified Delphi Investigation of Exercise Science in Physical Education Teacher Education

Round II of the Study 


\title{
(ON LETTERHEAD STATIONARY)
}

\author{
(PANEL MEMBER NAME) \\ (MAILING ADDRESS) \\ (CITY, STATE, ZIP CODE)
}

(DATE)

Dear

Thank you again for agreeing to be a participant in my study titled a Modified Delphi

Investigation of Exercise Science in Physical Education Teacher Education. In this round, your task will be to rate each item in the enclosed questionnaire a second time. As additional

information, you will find the Delphi panel's average ratings for each item included on the survey. Your individual ratings of each item have also been provided for your consideration.

The items that you are evaluating were adapted from the American College of Sports Medicine $(2000)^{1}$ and modified with the permission of the copyright owner, Lippincott Williams \& Wilkins. Please read the instructions that have been provided prior to completing the survey. If you have any questions, contact me at (715) 836-3722 or bulgersm@uwec.edu.

Your completion of this second round questionnaire is essential for the continued success of the study. While it is critical that you complete this second and final round of the rating process, your participation is entirely voluntary and you do not have to respond to every item on the questionnaire. You may be assured of complete confidentiality regarding all of your responses. Final results of the study will be reported to the participants in the form of an executive summary.

If possible, please return the completed survey to me within four weeks of receiving it. Additionally, refrain from discussing your opinions regarding the questionnaire with other members of the panel you may come in contact with. Anonymity of response represents one of the defining characteristics of the Delphi methodology. Use the return envelope that I have provided for your convenience. Thanks again for your assistance and cooperation.

Sincerely,

Sean M. Bulger

Assistant Professor

\footnotetext{
${ }^{1}$ ACSM. (2000). ACSM's Guidelines for Exercise Testing and Prescription ( $6^{\text {th }}$ edition). Baltimore, MD: Lippincott Williams \& Wilkins.
} 


\title{
Modified Delphi Investigation of Exercise Science in Physical Education Teacher Education
}

\author{
Round II Questionnaire
}

November 2003

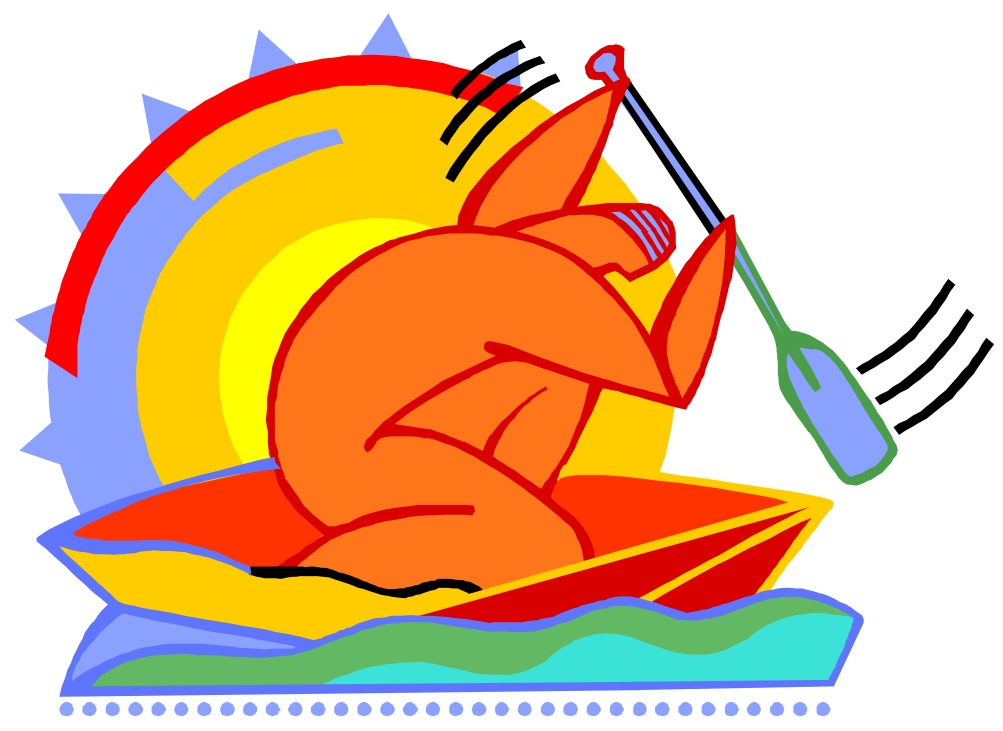

Sean M. Bulger, Doctoral Candidate

West Virginia University

School of Physical Education 


\section{Introduction}

The questionnaire you have agreed to complete will be used to generate a consensus of expert opinion regarding the critical theoretical and applied exercise science competencies that prospective physical educators need to learn within the physical education teacher education curriculum. The Delphi Method will be employed to address this primary research question. The Delphi Method is a research protocol that involves (a) the identification and selection of a small panel of experts on a particular topic, (b) the use of multiple rounds of surveying as a means to collect expert opinion, and (c) the attainment of group consensus through the provision of regular feedback.

Your expertise and judgment remains central to the success of this project. Due to the nature of both the involved research question and the Delphi Method, the list of potential survey items I am asking you to evaluate is rather extensive. The items that you are evaluating were adapted and modified from the American College of Sports Medicine (2000) ${ }^{1}$ and reproduced with the permission of the copyright owner, Lippincott Williams \& Wilkins.

Please know that your commitment to the timely completion of this project is greatly appreciated.

\section{Table of Contents}

Instructions................................................................................. ii

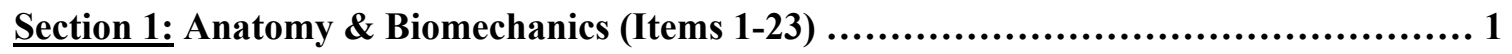

Section 2: Exercise Physiology (Items 24-58) ......................................... 3

Section 3: Human Development \& Aging (Items 59-69) .................................. 6

Section 4: Pathophysiology/Risk Factors (Items 70-83) ................................. 7

Section 5: Human Behavior \& Psychology (Items 84-100) ............................... 9

Section 6: Health Appraisal \& Fitness Testing (Items 101-132) .............................. 11

Section 7: Safety, Injury Prevention, and Emergency Care (Items 133-148) ................ 13

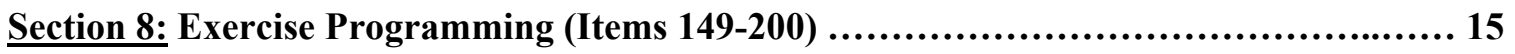

Section 9: Nutrition \& Weight Management (Items 201-218) ............................ 19

Section 10: Program \& Administration/Management (Items 219-222) ................... 21

Section 11: Recommended Instructional Methods........................................22

\footnotetext{
${ }^{1}$ ACSM. (2000). ACSM's Guidelines for Exercise Testing and Prescription ( $6^{\text {th }}$ edition). Baltimore, MD: Lippincott Williams \& Wilkins.
} 


\section{To: Exercise Science Delphi Panel Members}

\section{From: Sean M. Bulger, Doctoral Candidate \\ RE: Instructions for Completing the Round II Survey Instrument}

Thank you very much for your assistance in completing this research project. It is hoped that this study will result in the generation of a comprehensive list of critical theoretical and applied exercise science competencies that are recommended for inclusion in the physical education teacher education curriculum. Using the following set of instructions, please complete the attached questionnaire and return it to me within the next four weeks.

$\square$ Rate each of the questionnaire items separately in the areas of IMPORTANCE and RELEVANCE. For the purpose of this study, IMPORTANCE will refer to the item's value as a theoretical underpinning of the physical education profession. RELEVANCE will refer to the item's practical or applied value to the K-12 physical educator.

Please note that the average GROUP RATING (GR) for each item has been included to provide you with some additional information concerning the relationship between your INDIVIDUAL RATING (IR) from the previous round and the group mean response.

A rating of (5) or (4) in BOTH IMPORTANCE and RELEVANCE means that you consider the item to be critical in the preparation of prospective K-12 physical education teachers.

$\square$ A rating of (3) means you are neutral or undecided regarding the IMPORTANCE and RELEVANCE of the item.

A rating of (2) or (1) on EITHER IMPORTANCE or RELEVANCE means the item is not critical for the preparation of prospective K-12 physical education teachers.

If you choose not to respond to a particular item, please leave it blank.

A At the end of the survey, you will find an additional set of questions regarding recommended instructional strategies for the delivery of exercise physiology content in the physical education teacher education curriculum. Please complete this part of the survey as well. Completion of this round concludes your participation in the study.

Thanks again and please feel free to contact me directly with any questions at (715) 836-3722 or bulgersm@uwec.edu

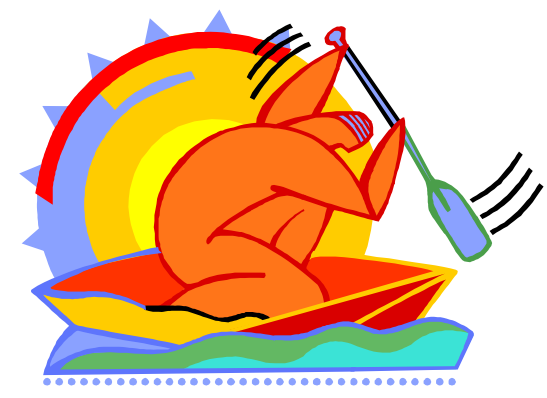




\section{Modified Delphi Investigation of Exercise Science in Physical Education Teacher Education} Round II Questionnaire

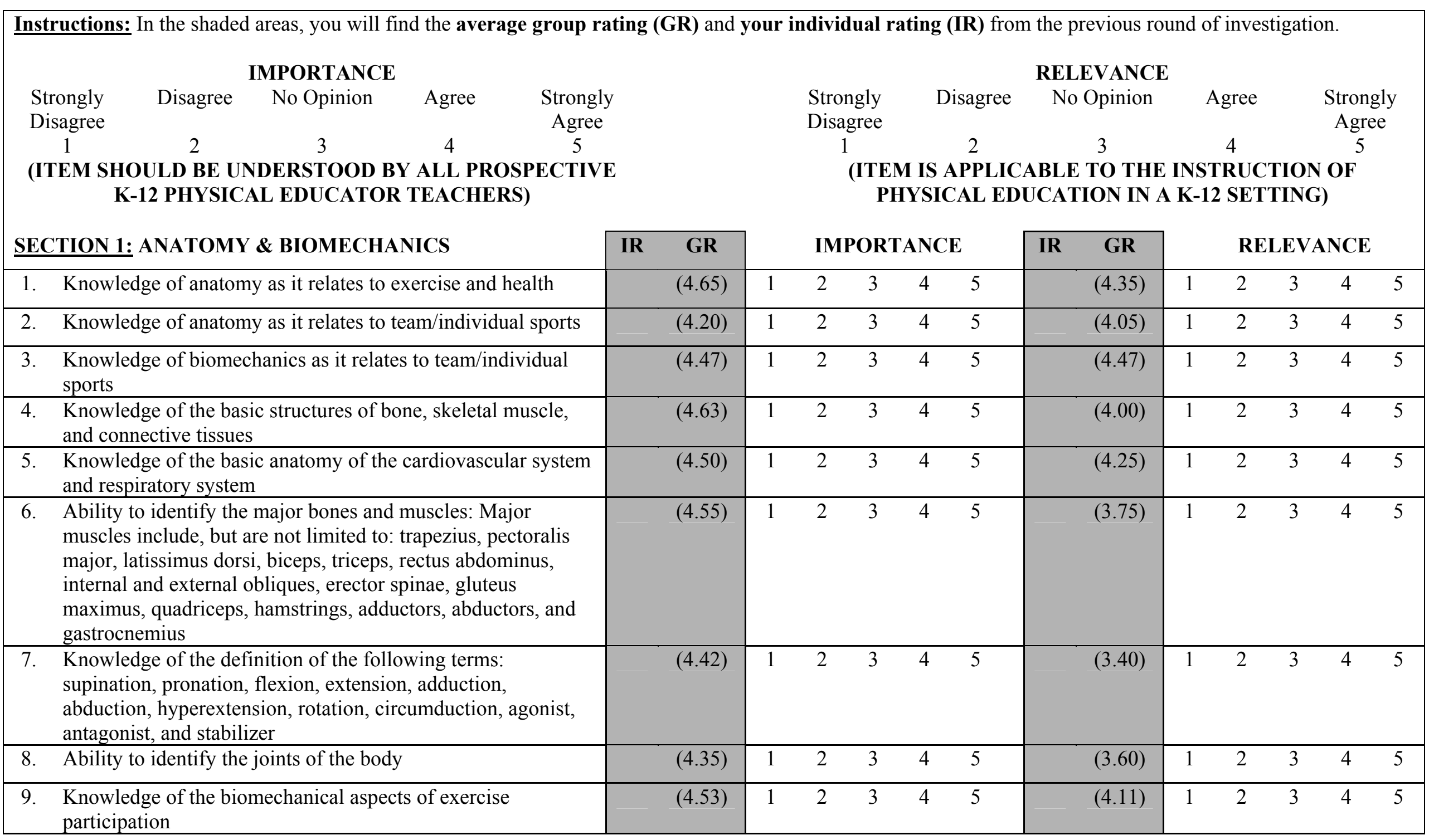




\begin{tabular}{|c|c|c|c|c|c|c|c|c|c|c|c|c|}
\hline $\begin{array}{l}\text { 10. Knowledge to identify the plane in which each muscle action } \\
\text { occurs }\end{array}$ & $(3.50)$ & 1 & 2 & 3 & 4 & 5 & $(2.80)$ & 1 & 2 & 3 & 4 & 5 \\
\hline $\begin{array}{l}\text { 11. Knowledge of the interrelationships among center of gravity, } \\
\text { base of support, balance, stability, and proper spinal } \\
\text { alignment }\end{array}$ & $(4.20)$ & 1 & 2 & 3 & 4 & 5 & $(3.89)$ & 1 & 2 & 3 & 4 & 5 \\
\hline $\begin{array}{l}\text { 12. Ability to describe the following curvatures of the spine: } \\
\text { lordosis, scoliosis, and kyphosis }\end{array}$ & $(3.65)$ & 1 & 2 & 3 & 4 & 5 & $(3.30)$ & 1 & 2 & 3 & 4 & 5 \\
\hline $\begin{array}{l}\text { 13. Knowledge of and skill to demonstrate exercises designed to } \\
\text { enhance muscular strength and/or endurance of specific major } \\
\text { muscle groups }\end{array}$ & $(4.75)$ & 1 & 2 & 3 & 4 & 5 & $(4.65)$ & 1 & 2 & 3 & 4 & 5 \\
\hline $\begin{array}{l}\text { 14. Knowledge of and skill to demonstrate exercise for enhancing } \\
\text { musculoskeletal flexibility }\end{array}$ & $(4.70)$ & 1 & 2 & 3 & 4 & 5 & $(4.60)$ & 1 & 2 & 3 & 4 & 5 \\
\hline 15. Knowledge to describe the myotatic stretch reflex & $(3.65)$ & 1 & 2 & 3 & 4 & 5 & $(2.90)$ & 1 & 2 & 3 & 4 & 5 \\
\hline $\begin{array}{l}\text { 16. Knowledge to identify the primary action and joint range of } \\
\text { motion for each major muscle group }\end{array}$ & $(4.10)$ & 1 & 2 & 3 & 4 & 5 & $(3.80)$ & 1 & 2 & 3 & 4 & 5 \\
\hline 17. Knowledge of functional anatomy and biomechanics & $(4.50)$ & 1 & 2 & 3 & 4 & 5 & $(4.28)$ & 1 & 2 & 3 & 4 & 5 \\
\hline $\begin{array}{l}\text { 18. Knowledge of the structure and ability to describe movements } \\
\text { for the major joints of the body }\end{array}$ & $(4.40)$ & 1 & 2 & 3 & 4 & 5 & $(3.85)$ & 1 & 2 & 3 & 4 & 5 \\
\hline $\begin{array}{l}\text { 19. Ability to locate the anatomic landmarks for palpation of } \\
\text { peripheral pulses }\end{array}$ & $(4.05)$ & 1 & 2 & 3 & 4 & 5 & $(3.60)$ & 1 & 2 & 3 & 4 & 5 \\
\hline $\begin{array}{l}\text { 20. Ability to locate the brachial artery and correctly place the } \\
\text { cuff and stethoscope in position for blood pressure } \\
\text { measurement }\end{array}$ & $(3.90)$ & 1 & 2 & 3 & 4 & 5 & $(3.25)$ & 1 & 2 & 3 & 4 & 5 \\
\hline $\begin{array}{l}\text { 21. Ability to locate common sites for measurement of skinfold } \\
\text { thickness and circumferences (for determination of body } \\
\text { composition and waist-hip ratio) }\end{array}$ & $(4.60)$ & 1 & 2 & 3 & 4 & 5 & $(4.40)$ & 1 & 2 & 3 & 4 & 5 \\
\hline $\begin{array}{l}\text { 22. Knowledge of the biomechanical principles that underlie } \\
\text { performance of the following activities: walking, jogging, } \\
\text { running, swimming, cycling, weight lifting, and carrying or } \\
\text { moving objects }\end{array}$ & $(4.55)$ & 1 & 2 & 3 & 4 & 5 & $(4.25)$ & 1 & 2 & 3 & 4 & 5 \\
\hline $\begin{array}{l}\text { 23. Knowledge of the influence of weight-bearing activity in } \\
\text { childhood and adolescence on optimal bone growth and } \\
\text { development }\end{array}$ & $(4.50)$ & 1 & 2 & 3 & 4 & 5 & $(4.15)$ & 1 & 2 & 3 & 4 & 5 \\
\hline
\end{tabular}




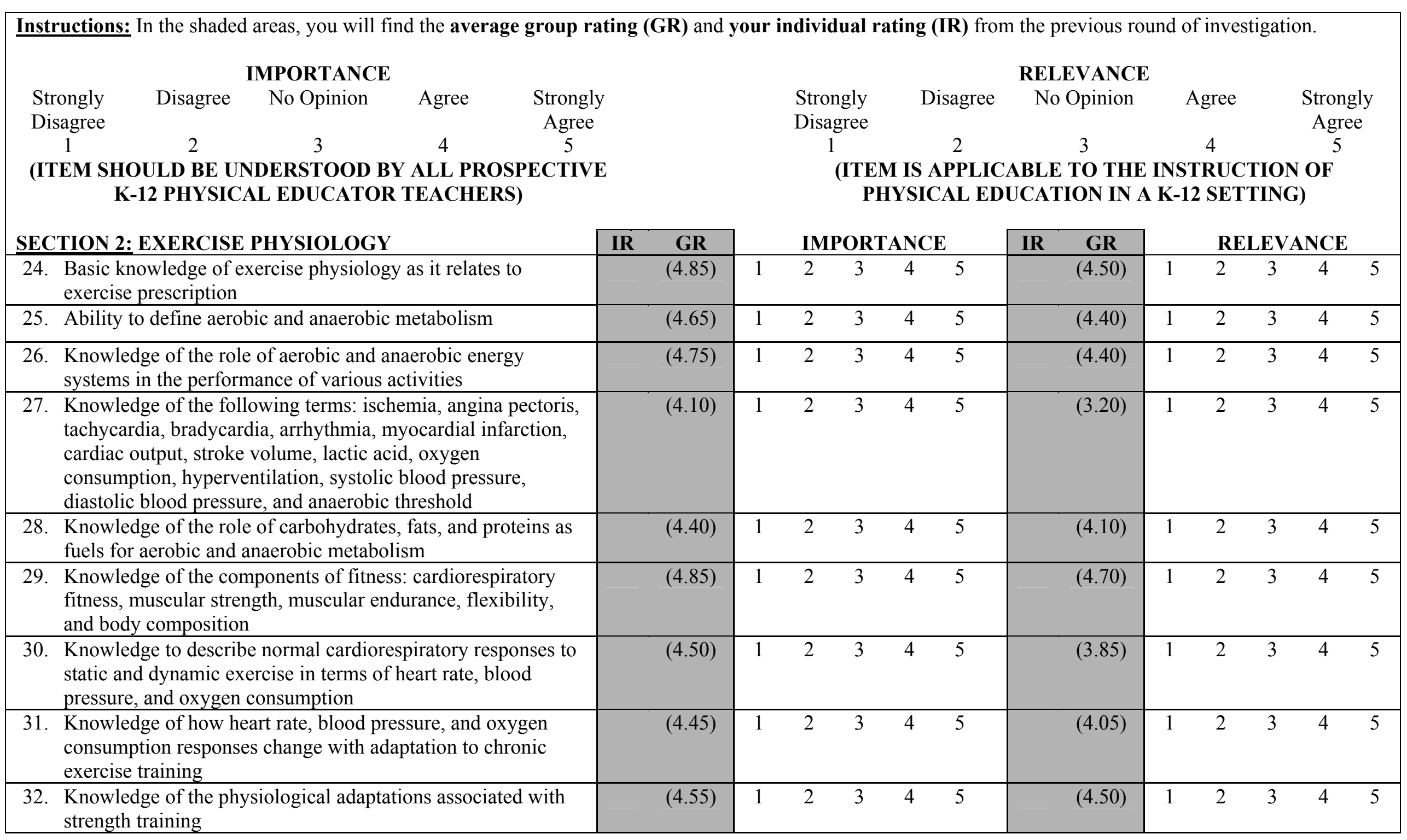




\begin{tabular}{|c|c|c|c|c|c|c|c|c|c|c|c|c|c|}
\hline & $\begin{array}{l}\text { Ability to identify and apply to both groups and individuals } \\
\text { methods used to monitor exercise intensity, including heart } \\
\text { rate and rating of perceived exertion }\end{array}$ & $(4.60)$ & 1 & 2 & 3 & 4 & 5 & $(4.60)$ & 1 & 2 & 3 & 4 & 5 \\
\hline 34. & $\begin{array}{l}\text { Knowledge of the physiological principles related to warm-up } \\
\text { and cool-down }\end{array}$ & $(4.55)$ & 1 & 2 & 3 & 4 & 5 & $(4.55)$ & 1 & 2 & 3 & 4 & 5 \\
\hline 35. & $\begin{array}{l}\text { Knowledge of the common theories of muscle fatigue and } \\
\text { delayed onset muscle soreness (DOMS) }\end{array}$ & $(4.10)$ & 1 & 2 & 3 & 4 & 5 & $(3.75)$ & 1 & 2 & 3 & 4 & 5 \\
\hline 36. & $\begin{array}{l}\text { Knowledge of exercise physiology including the role of } \\
\text { aerobic and anaerobic metabolism, muscle physiology, } \\
\text { cardiovascular physiology, and respiratory physiology at rest } \\
\text { and during exercise. In addition, demonstrate an } \\
\text { understanding of the components of physical fitness, the } \\
\text { effects of aerobic and strength and/or resistance training on } \\
\text { the fitness components and the effects of chronic disease }\end{array}$ & $(4.65)$ & 1 & 2 & 3 & 4 & 5 & $(4.35)$ & 1 & 2 & 3 & 4 & 5 \\
\hline 37. & $\begin{array}{l}\text { Knowledge of the physiological adaptations that occur at rest } \\
\text { and during submaximal and maximal exercise following } \\
\text { chronic aerobic and anaerobic exercise training }\end{array}$ & $(4.30)$ & 1 & 2 & 3 & 4 & 5 & $(3.90)$ & 1 & 2 & 3 & 4 & 5 \\
\hline 38. & $\begin{array}{l}\text { Knowledge of the differences in cardiorespiratory response to } \\
\text { acute graded exercise between conditioned and unconditioned } \\
\text { individuals }\end{array}$ & $(4.00)$ & 1 & 2 & 3 & 4 & 5 & $(3.45)$ & 1 & 2 & 3 & 4 & 5 \\
\hline & $\begin{array}{l}\text { Knowledge of the structure of the skeletal muscle fiber and } \\
\text { the basic mechanism of contraction }\end{array}$ & $(3.85)$ & 1 & 2 & 3 & 4 & 5 & $(3.10)$ & 1 & 2 & 3 & 4 & 5 \\
\hline 40. & Knowledge of the characteristics of fast and slow twitch fibers & $(3.90)$ & 1 & 2 & 3 & 4 & 5 & $(3.40)$ & 1 & 2 & 3 & 4 & 5 \\
\hline & $\begin{array}{l}\text { Knowledge of the sliding filament theory of muscle } \\
\text { contraction }\end{array}$ & $(3.45)$ & 1 & 2 & 3 & $\overline{4}$ & 5 & $(2.55)$ & 1 & 2 & 3 & $\overline{4}$ & 5 \\
\hline 42. & $\begin{array}{l}\text { Knowledge of twitch, summation, and tetanus with respect to } \\
\text { muscle contraction }\end{array}$ & $(3.20)$ & 1 & 2 & 3 & 4 & 5 & $(2.40)$ & 1 & 2 & 3 & 4 & 5 \\
\hline 43. & $\begin{array}{l}\text { Ability to discuss the physiological principles involved in } \\
\text { promoting gains in muscular strength and endurance }\end{array}$ & $(4.55)$ & 1 & 2 & 3 & 4 & 5 & $(4.50)$ & 1 & 2 & 3 & 4 & 5 \\
\hline 44. & $\begin{array}{l}\text { Ability to define muscular fatigue as it relates to task, } \\
\text { intensity, duration, and the accumulative effects of exercise }\end{array}$ & $(4.20)$ & 1 & 2 & 3 & 4 & 5 & $(3.80)$ & 1 & 2 & 3 & 4 & 5 \\
\hline 45. & $\begin{array}{l}\text { Knowledge of the relationship between the number of } \\
\text { repetitions, intensity, number of sets, and rest with regard to } \\
\text { strength training }\end{array}$ & $(4.55)$ & 1 & 2 & 3 & 4 & 5 & $(4.50)$ & 1 & 2 & 3 & 4 & 5 \\
\hline
\end{tabular}




\begin{tabular}{|c|c|c|c|c|c|c|c|c|c|c|c|c|c|}
\hline & $\begin{array}{l}\text { Knowledge of the basic properties of cardiac muscle and the } \\
\text { normal pathways of conduction in the heart }\end{array}$ & $(3.60)$ & 1 & 2 & 3 & 4 & 5 & $(2.55)$ & 1 & 2 & 3 & 4 & 5 \\
\hline 47. & $\begin{array}{l}\text { Knowledge of the response of the following variables to acute } \\
\text { exercise: heart rate, stroke volume, cardiac output, pulmonary } \\
\text { ventilation, tidal volume, respiratory rate, and arteriovenous } \\
\text { oxygen difference }\end{array}$ & $(3.75)$ & 1 & 2 & 3 & 4 & 5 & $(3.42)$ & 1 & 2 & 3 & 4 & 5 \\
\hline 48. & $\begin{array}{l}\text { Knowledge of the differences in the cardiorespiratory } \\
\text { responses to static exercise compared with dynamic exercise, } \\
\text { including possible hazards and contraindications }\end{array}$ & $(3.95)$ & 1 & 2 & 3 & 4 & 5 & $(3.95)$ & 1 & 2 & 3 & 4 & 5 \\
\hline 49. & $\begin{array}{l}\text { Ability to describe how each of the following differs from the } \\
\text { normal condition: premature atrial contractions and premature } \\
\text { ventricular contractions }\end{array}$ & $(2.90)$ & 1 & 2 & 3 & 4 & 5 & $(2.15)$ & 1 & 2 & 3 & 4 & 5 \\
\hline 50. & $\begin{array}{l}\text { Knowledge of blood pressure responses associated with acute } \\
\text { exercise, including changes in body position }\end{array}$ & $(3.75)$ & 1 & 2 & 3 & 4 & 5 & $(3.30)$ & 1 & 2 & 3 & 4 & 5 \\
\hline 51. & $\begin{array}{l}\text { Knowledge of and ability to describe the implications of } \\
\text { ventilatory threshold (anaerobic threshold) as it relates to } \\
\text { exercise training and cardiorespiratory assessment }\end{array}$ & $(3.75)$ & 1 & 2 & 3 & 4 & 5 & $(3.25)$ & 1 & 2 & 3 & 4 & 5 \\
\hline 52. & $\begin{array}{l}\text { Knowledge of and ability to describe the physiological } \\
\text { adaptations of the respiratory system that occur at rest and } \\
\text { during submaximal and maximal exercise following chronic } \\
\text { aerobic and anaerobic training }\end{array}$ & $(3.85)$ & 1 & 2 & 3 & 4 & 5 & $(3.30)$ & 1 & 2 & 3 & 4 & 5 \\
\hline 53. & $\begin{array}{l}\text { Ability to describe how each of the following differs from the } \\
\text { normal condition: dyspnea, hypoxia, and hypoventilation }\end{array}$ & $(3.35)$ & 1 & 2 & 3 & 4 & 5 & $(2.75)$ & 1 & 2 & 3 & 4 & 5 \\
\hline 54. & $\begin{array}{l}\text { Knowledge of and ability to discuss the physiological basis of } \\
\text { the major components of physical fitness: flexibility, } \\
\text { cardiovascular fitness, muscular strength, muscular } \\
\text { endurance, and body composition }\end{array}$ & $(4.85)$ & 1 & 2 & 3 & 4 & 5 & $(4.70)$ & 1 & 2 & 3 & 4 & 5 \\
\hline 55. & $\begin{array}{l}\text { Ability to explain how the principle of specificity relates to } \\
\text { the components of fitness }\end{array}$ & $(4.55)$ & 1 & 2 & 3 & 4 & 5 & $(4.60)$ & 1 & 2 & 3 & 4 & 5 \\
\hline 56. & $\begin{array}{l}\text { Ability to explain the concept of detraining or reversibility of } \\
\text { conditioning and its implications in fitness programs }\end{array}$ & $(4.15)$ & 1 & 2 & 3 & 4 & 5 & $(4.20)$ & 1 & 2 & 3 & 4 & 5 \\
\hline 57. & $\begin{array}{l}\text { Ability to discuss the physical and psychological signs of } \\
\text { overtraining and provide recommendations for these problems }\end{array}$ & $(4.05)$ & 1 & 2 & 3 & 4 & 5 & $(3.75)$ & 1 & 2 & 3 & 4 & 5 \\
\hline 58. & $\begin{array}{l}\text { Ability to describe the physiological and metabolic responses } \\
\text { to exercise associated with chronic disease (heart disease, } \\
\text { hypertension, diabetes mellitus, and pulmonary disease) }\end{array}$ & $(3.75)$ & 1 & 2 & 3 & 4 & 5 & $(3.50)$ & 1 & 2 & 3 & 4 & 5 \\
\hline
\end{tabular}




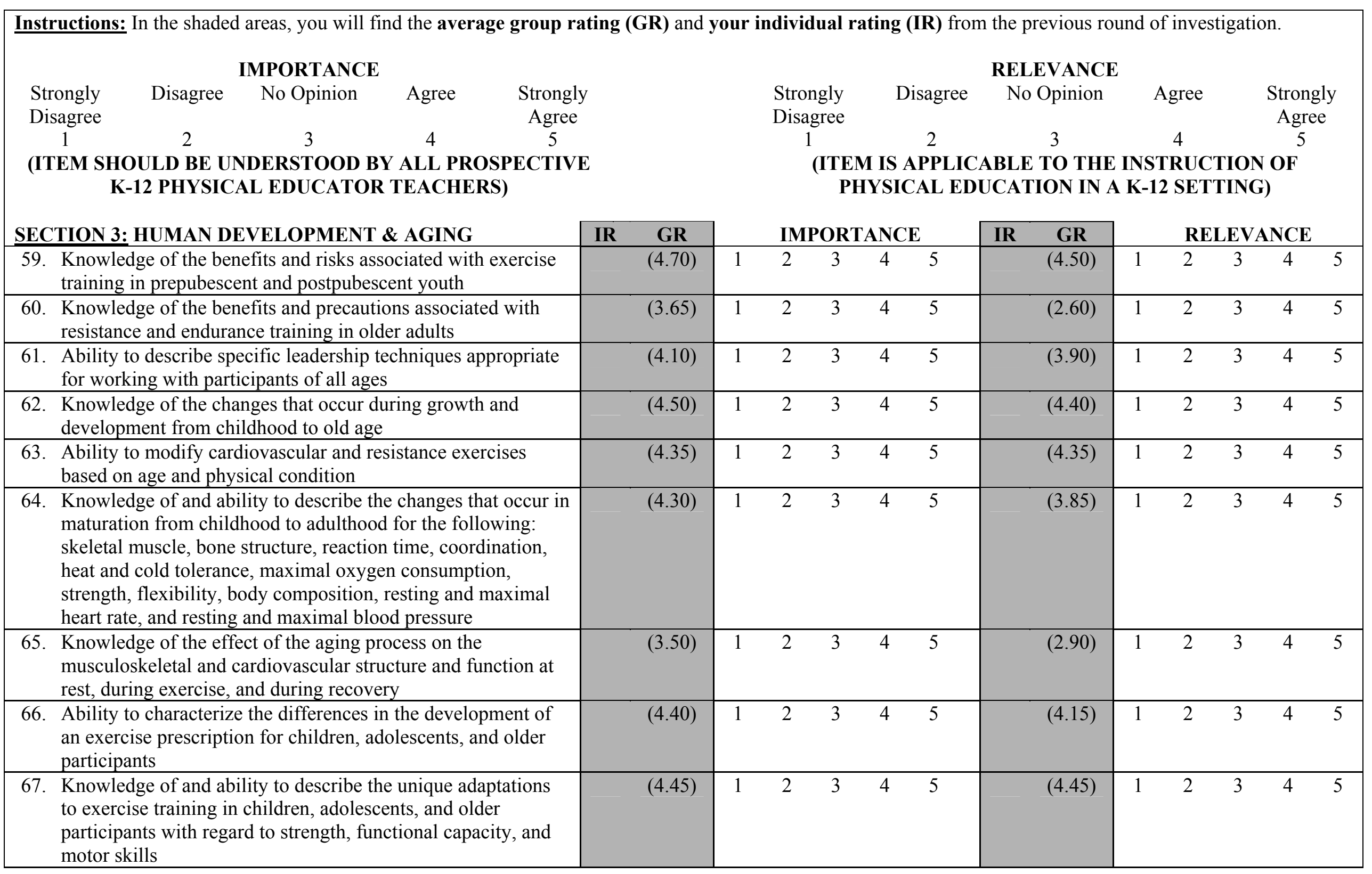


68. Knowledge of the common orthopedic and cardiovascular considerations for older participants and the ability to describe modifications in exercise prescription that are indicated

69. Knowledge of the health-related benefits of regular physical activity for people of all ages

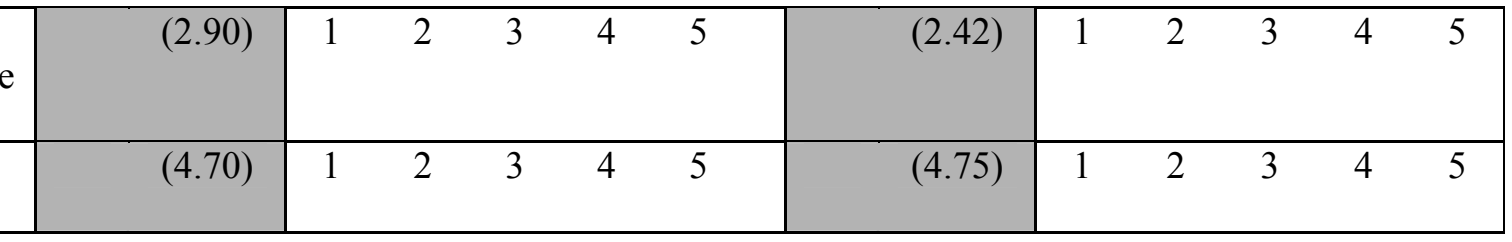

Instructions: In the shaded areas, you will find the average group rating (GR) and your individual rating (IR) from the previous round of investigation.

IMPORTANCE

Strongly

No Opinion

Strongly
Agree

3
4

\section{RELEVANCE}

Disagree 2

ITEM SHOULD BE UNDERSTOOD BY ALL PROSPECTIVE K-12 PHYSICAL EDUCATOR TEACHERS)

5
No Opinion

Strongly Disagree
3

Agree Strongly 2
(ITEM IS APPLICABLE TO THE INSTRUCTION OF PHYSICAL EDUCATION IN A K-12 SETTING)

\section{SECTION 4: PATHOPHYSIOLOGY/RISK FACTORS}

70. Knowledge of cardiovascular, respiratory, metabolic, and musculoskeletal risk factors that may require further evaluation by medical or allied health professionals before participation in physical activity

71. Ability to determine those risk factors that may be favorably modified by physical activity habits

72. Knowledge to define the following terms: total cholesterol (TC), high-density lipoprotein cholesterol (HDL-C), TC/HDL-C ratio, low-density lipoprotein cholesterol (LDLC), triglycerides, hypertension, and artherosclerosis

73. Knowledge of plasma cholesterol levels for adults as recommended by the National Cholesterol Education Program (NCEP II)

74. Knowledge of the pathophysiology of atherosclerosis and how this process is influenced by physical activity

75. Knowledge of the risk factor concept of coronary artery disease (CAD) and the influence of heredity and lifestyle on the development of CAD

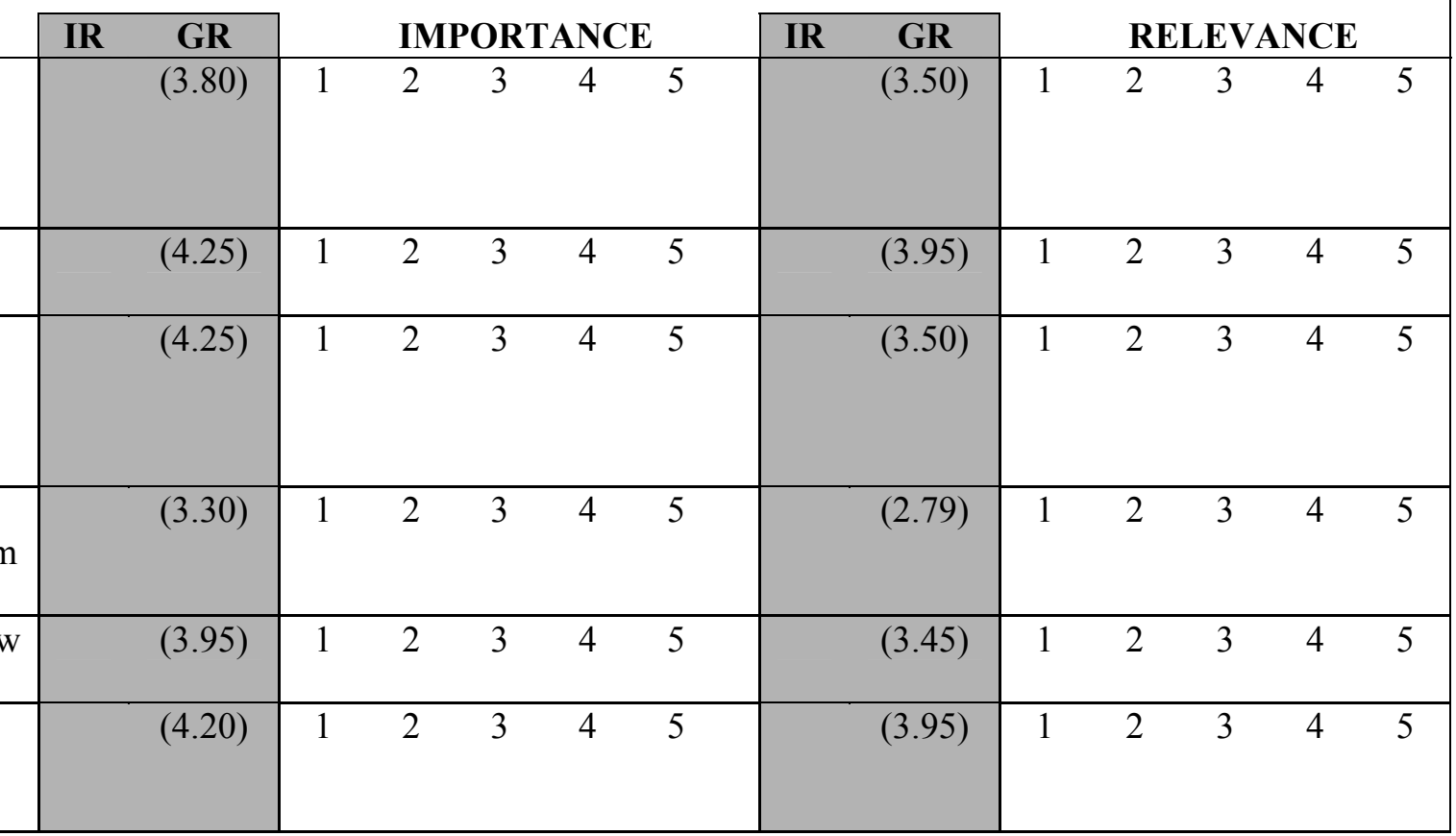




\begin{tabular}{|c|c|c|c|c|c|c|c|c|c|c|c|c|c|}
\hline & $\begin{array}{l}\text { Knowledge of the artherosclerotic process, the factors } \\
\text { involved in its genesis and progression, and the potential role } \\
\text { of exercise training in treatment }\end{array}$ & $(3.50)$ & 1 & 2 & 3 & 4 & 5 & $(2.85)$ & 1 & 2 & 3 & 4 & 5 \\
\hline 77. & $\begin{array}{l}\text { Ability to discuss in detail how lifestyle factors, including } \\
\text { nutrition, physical activity, and heredity, influence lipid and } \\
\text { lipoprotein profiles }\end{array}$ & $(4.15)$ & 1 & 2 & 3 & 4 & 5 & $(4.00)$ & 1 & 2 & 3 & 4 & 5 \\
\hline 78. & $\begin{array}{l}\text { Knowledge of cardiovascular risk factors or conditions that } \\
\text { may require consultation with medical personnel before } \\
\text { testing or training, including inappropriate changes in resting } \\
\text { or exercise heart rate and blood pressure, new onset } \\
\text { discomfort in chest, neck, shoulder, or arm, changes in the } \\
\text { pattern of discomfort during rest or exercise, fainting or dizzy } \\
\text { spells, and claudication }\end{array}$ & $(4.20)$ & 1 & 2 & 3 & 4 & 5 & $(3.85)$ & 1 & 2 & 3 & 4 & 5 \\
\hline 79. & $\begin{array}{l}\text { Knowledge of the respiratory risk factors or conditions that } \\
\text { may require consultation with medical personnel before } \\
\text { testing or training, including asthma, exercise-induced } \\
\text { bronchospasm, extreme breathlessness at rest or during } \\
\text { exercise, bronchitis, and emphysema }\end{array}$ & $(4.05)$ & 1 & 2 & 3 & 4 & 5 & $(3.85)$ & 1 & 2 & 3 & 4 & 5 \\
\hline 80. & $\begin{array}{l}\text { Knowledge of the metabolic risk factors or conditions that } \\
\text { may require consultation with medical personnel before } \\
\text { testing or training, including bodyweight more than } 20 \% \\
\text { above optimal, BMI }>30 \text {, thyroid disease, diabetes or glucose } \\
\text { intolerance, and hypoglycemia }\end{array}$ & $(3.95)$ & 1 & 2 & 3 & 4 & 5 & $(3.65)$ & 1 & 2 & 3 & 4 & 5 \\
\hline 81. & $\begin{array}{l}\text { Knowledge of the musculoskeletal risk factors or conditions } \\
\text { that may require consultation with medical personnel before } \\
\text { testing or training, including acute or chronic back pain, } \\
\text { osteoarthritis, rheumatoid arthritis, osteoporosis, tendonitis, } \\
\text { and low back pain }\end{array}$ & $(3.80)$ & 1 & 2 & 3 & 4 & 5 & $(3.10)$ & 1 & 2 & 3 & 4 & 5 \\
\hline 82. & $\begin{array}{l}\text { Knowledge of persons with physical and mental disabilities } \\
\text { and the potential influence on risk for chronic degenerative } \\
\text { disease }\end{array}$ & $(3.65)$ & 1 & 2 & 3 & 4 & 5 & $(3.25)$ & 1 & 2 & 3 & 4 & 5 \\
\hline 83. & $\begin{array}{l}\text { Knowledge of the factors that increase risk for osteoporosis } \\
\text { and the preventive measures that can be followed to decrease } \\
\text { risk }\end{array}$ & $(3.80)$ & 1 & 2 & 3 & 4 & 5 & $(3.25)$ & 1 & 2 & 3 & 4 & 5 \\
\hline
\end{tabular}




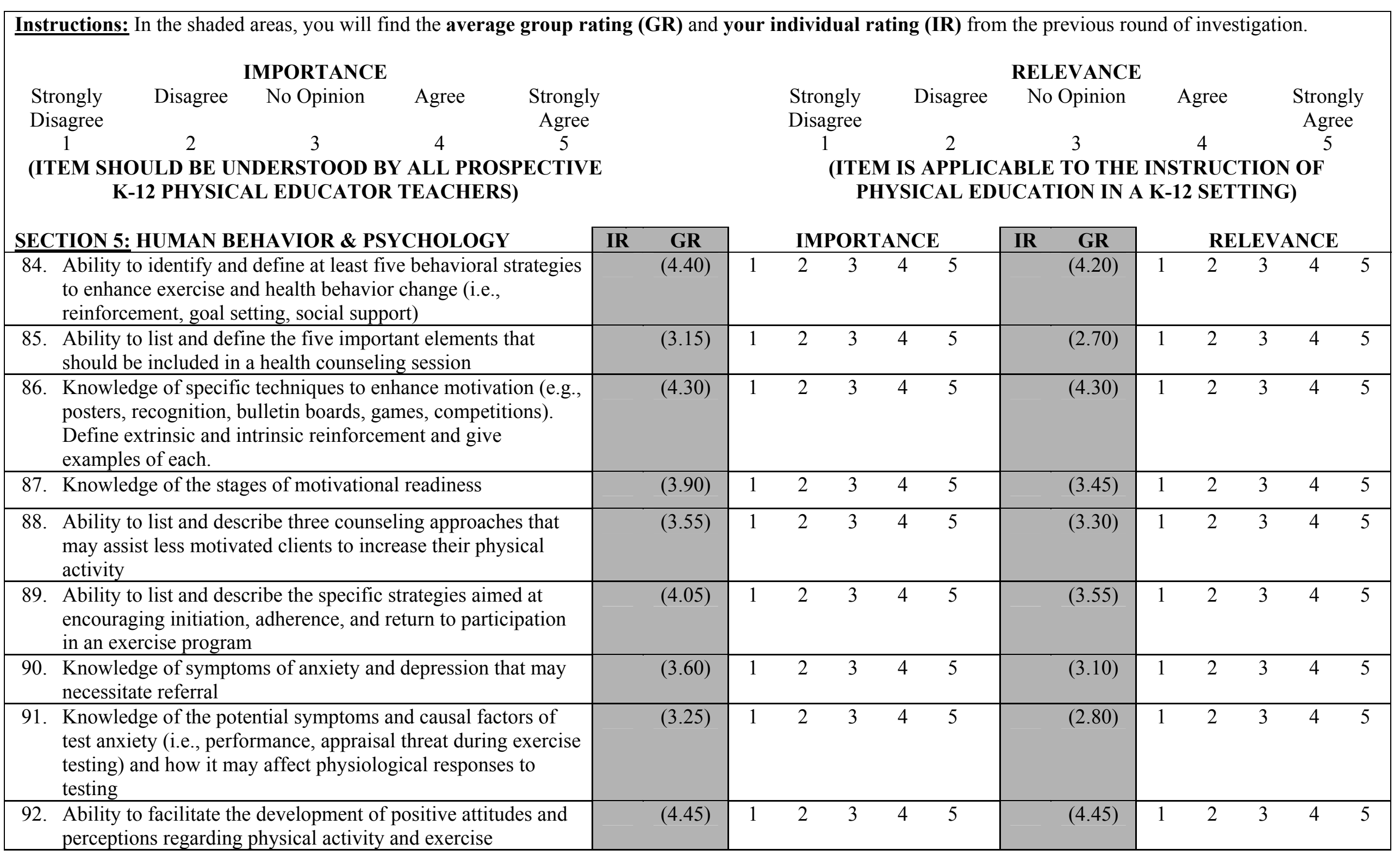




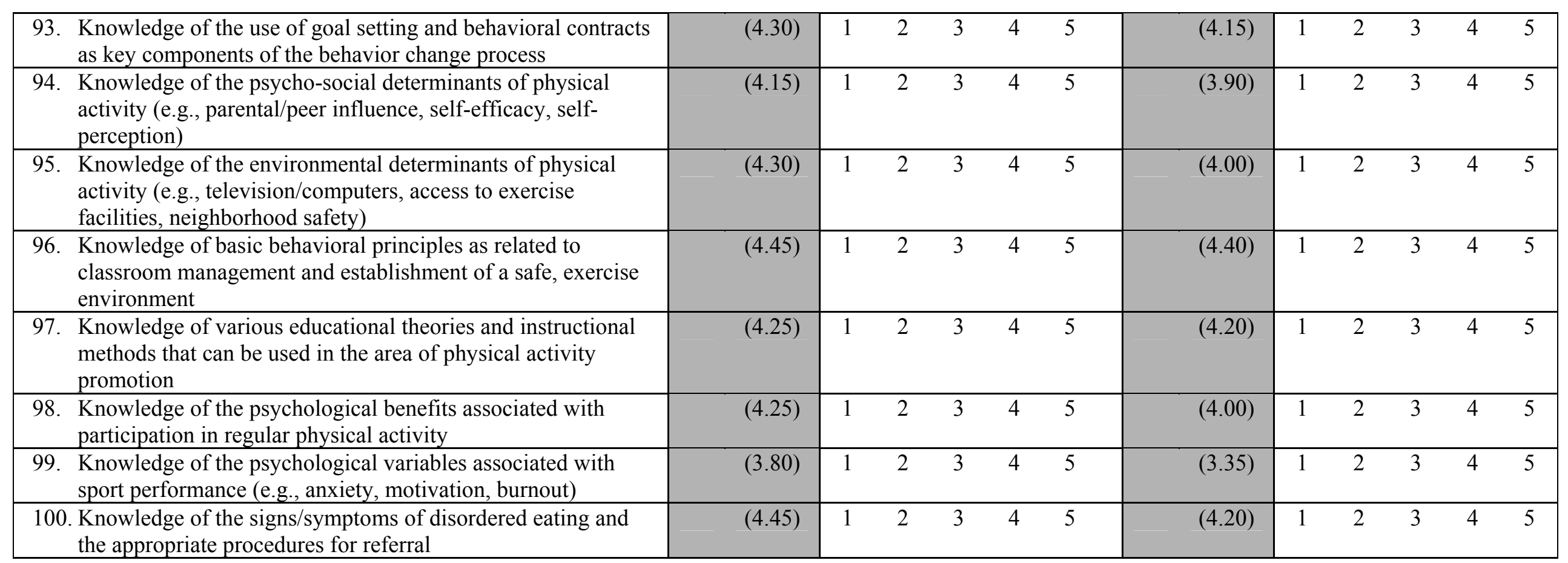
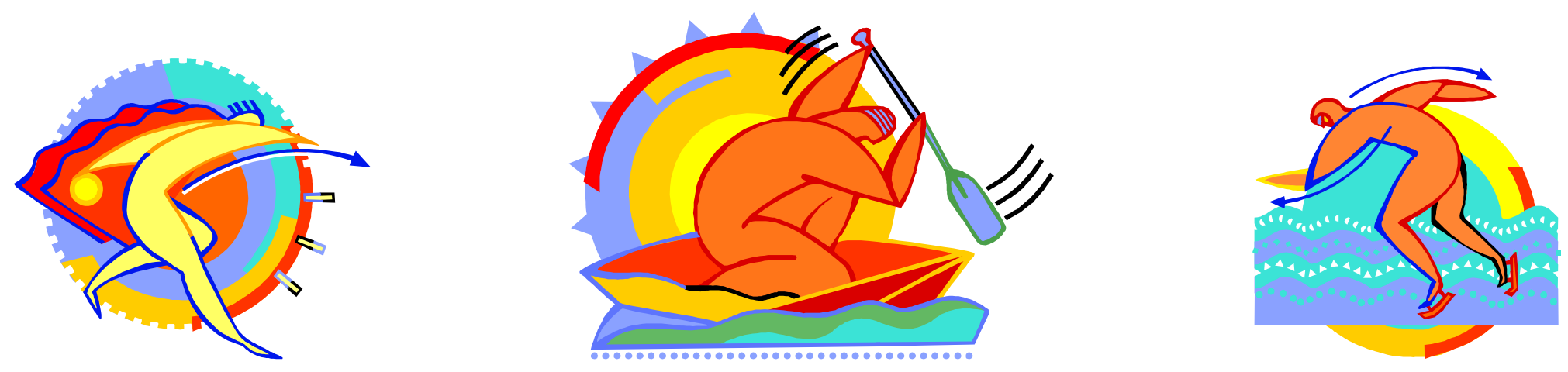

\section{Please continue on the next page...}




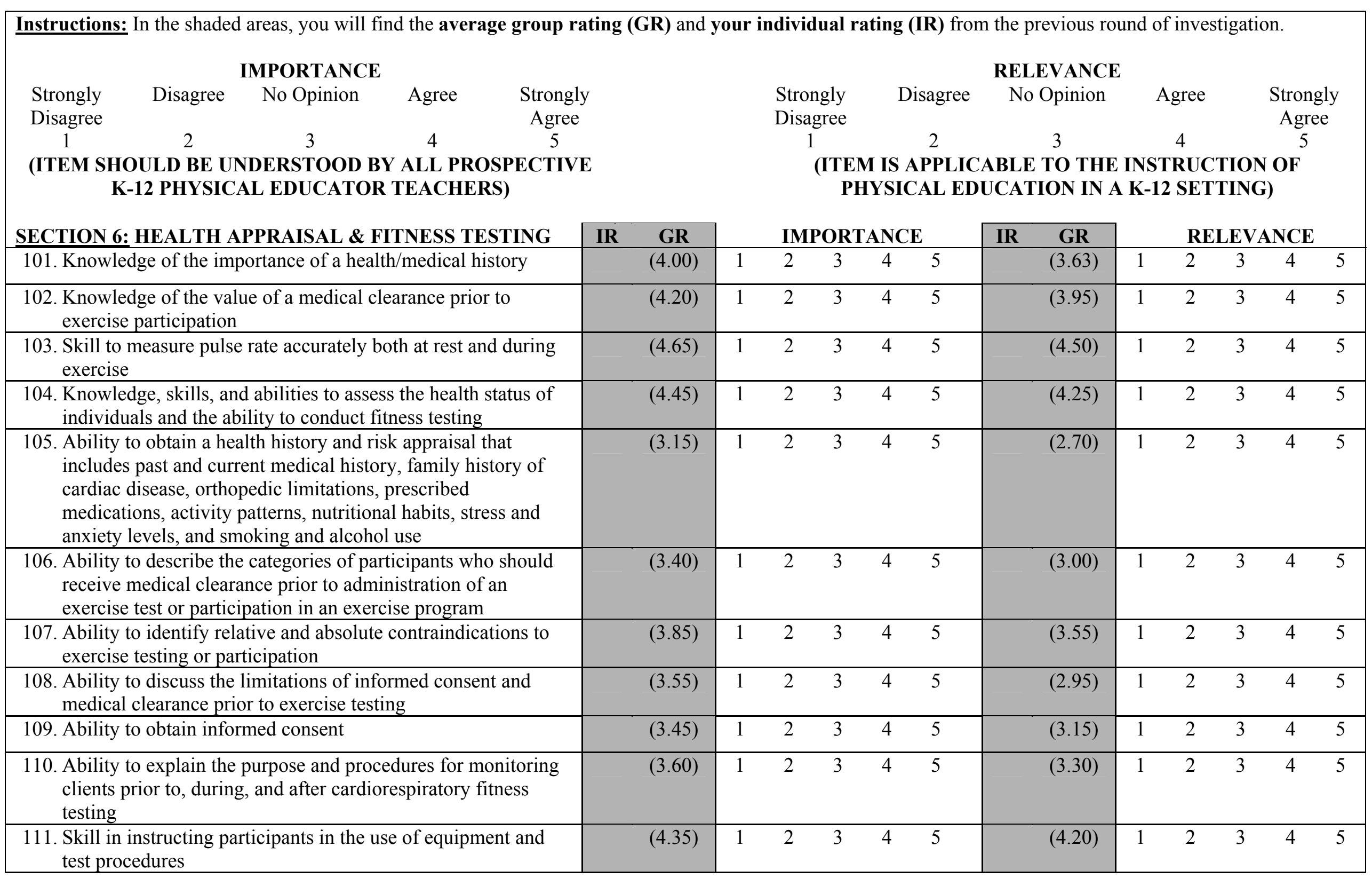




\begin{tabular}{|c|c|c|c|c|c|c|c|c|c|c|c|c|}
\hline $\begin{array}{l}\text { 112. Ability to describe the purpose of testing, select an } \\
\text { appropriate submaximal or maximal protocol, and conduct an } \\
\text { assessment of cardiovascular fitness on the cycle ergometer or } \\
\text { the treadmill }\end{array}$ & $(3.25)$ & 1 & 2 & 3 & 4 & 5 & $(2.35)$ & 1 & 2 & 3 & 4 & 5 \\
\hline $\begin{array}{l}\text { 113. Skill in accurately measuring heart rate, blood pressure, and } \\
\text { obtaining rating of perceived exertion (RPE) at rest and } \\
\text { during exercise according to established guidelines }\end{array}$ & $(4.25)$ & 1 & 2 & 3 & 4 & 5 & $(3.65)$ & 1 & 2 & 3 & 4 & 5 \\
\hline $\begin{array}{l}\text { 114. Ability to locate and measure skinfold sites, skeletal } \\
\text { diameters, and girth measurements used for estimating body } \\
\text { composition }\end{array}$ & $(4.35)$ & 1 & 2 & 3 & 4 & 5 & $(4.00)$ & 1 & 2 & 3 & 4 & 5 \\
\hline $\begin{array}{l}\text { 115. Ability to describe the purpose of testing, select appropriate } \\
\text { protocols, and conduct assessments of muscular strength, } \\
\text { muscular endurance, and flexibility }\end{array}$ & $(4.50)$ & 1 & 2 & 3 & 4 & 5 & $(4.45)$ & 1 & 2 & 3 & 4 & 5 \\
\hline 116. Skill in various techniques of assessing body composition & $(4.05)$ & 1 & 2 & 3 & 4 & 5 & $(3.95)$ & 1 & 2 & 3 & 4 & 5 \\
\hline $\begin{array}{l}\text { 117. Knowledge of the advantages/disadvantages and limitations } \\
\text { of the various body composition techniques }\end{array}$ & $(4.25)$ & 1 & 2 & 3 & 4 & 5 & $(3.65)$ & 1 & 2 & 3 & 4 & 5 \\
\hline $\begin{array}{l}\text { 118. Ability to interpret information obtained from } \\
\text { cardiorespiratory fitness tests and muscular } \\
\text { strength/endurance, flexibility, and body composition tests for } \\
\text { apparently healthy individuals and those with stable disease }\end{array}$ & $(3.80)$ & 1 & 2 & 3 & 4 & 5 & $(3.65)$ & 1 & 2 & 3 & 4 & 5 \\
\hline $\begin{array}{l}\text { 119. Ability to identify appropriate criteria for terminating a fitness } \\
\text { evaluation and demonstrate proper procedures to be followed } \\
\text { after discontinuing such a test }\end{array}$ & $(3.75)$ & 1 & 2 & 3 & 4 & 5 & $(3.50)$ & 1 & 2 & 3 & 4 & 5 \\
\hline $\begin{array}{l}\text { 120. Ability to modify protocols and procedures for } \\
\text { cardiorespiratory fitness tests in children, adolescents, and } \\
\text { older adults }\end{array}$ & $(4.30)$ & 1 & 2 & 3 & 4 & 5 & $(4.15)$ & 1 & 2 & 3 & 4 & 5 \\
\hline $\begin{array}{l}\text { 121. Ability to conduct fitness appraisals for populations with } \\
\text { physical and mental disabilities (e.g., mental retardation) } \\
\text { which may require alternative testing techniques }\end{array}$ & $(3.70)$ & 1 & 2 & 3 & 4 & 5 & $(3.35)$ & 1 & 2 & 3 & 4 & 5 \\
\hline $\begin{array}{l}\text { 122. Knowledge of the steps needed to prepare children for } \\
\text { physical fitness testing (e.g., teaching test purpose, protocol, } \\
\text { pacing) }\end{array}$ & $(4.55)$ & 1 & 2 & 3 & 4 & 5 & $(4.60)$ & 1 & 2 & 3 & 4 & 5 \\
\hline $\begin{array}{l}\text { 123. Knowledge of the differences between norm and criterion } \\
\text { referenced fitness tests }\end{array}$ & $(4.35)$ & 1 & 2 & 3 & 4 & 5 & $(4.25)$ & 1 & 2 & 3 & 4 & 5 \\
\hline
\end{tabular}




\begin{tabular}{|c|c|c|c|c|c|c|c|c|c|c|c|c|}
\hline $\begin{array}{l}\text { 124. Knowledge of the role of physical fitness testing in achieving } \\
\text { national and state standards for physical education }\end{array}$ & $(4.35)$ & 1 & 2 & 3 & 4 & 5 & $(4.40)$ & 1 & 2 & 3 & 4 & 5 \\
\hline $\begin{array}{l}\text { 125. Ability to use heart rate monitors for monitoring intensity } \\
\text { during exercise }\end{array}$ & $(4.20)$ & 1 & 2 & 3 & 4 & 5 & $(4.15)$ & 1 & 2 & 3 & 4 & 5 \\
\hline $\begin{array}{l}\text { 126. Knowledge of the NIH definitions of obesity and overweight } \\
\text { using body mass index (BMI) }\end{array}$ & $(4.20)$ & 1 & 2 & 3 & 4 & 5 & $(3.85)$ & 1 & 2 & 3 & 4 & 5 \\
\hline $\begin{array}{l}\text { 127. Ability to use pedometers for monitoring physical activity } \\
\text { participation }\end{array}$ & $(4.20)$ & 1 & 2 & 3 & 4 & 5 & $(4.25)$ & 1 & 2 & 3 & 4 & 5 \\
\hline \multicolumn{13}{|c|}{$\begin{array}{l}\text { Knowledge of common drugs from each of the following classes of medications and describe the principal action and the effects on exercise testing and } \\
\text { prescription: }\end{array}$} \\
\hline 128. Bronchodilators & $(3.26)$ & 1 & 2 & 3 & 4 & 5 & $(3.17)$ & 1 & 2 & 3 & 4 & 5 \\
\hline 129. Hypoglycemics & $(3.11)$ & 1 & 2 & 3 & 4 & 5 & $(2.78)$ & 1 & 2 & 3 & 4 & 5 \\
\hline 130. Psychotropics & $(3.05)$ & 1 & 2 & 3 & 4 & 5 & $(2.61)$ & 1 & 2 & 3 & 4 & 5 \\
\hline 131. Vasodilators & $(3.11)$ & 1 & 2 & 3 & 4 & 5 & $(2.83)$ & 1 & 2 & 3 & 4 & 5 \\
\hline $\begin{array}{l}\text { 132. Ability to identify the effects of the following substances on } \\
\text { exercise response: antihistamines, tranquilizers, alcohol, diet } \\
\text { pills, cold tablets, caffeine, and nicotine }\end{array}$ & $(3.55)$ & 1 & 2 & 3 & 4 & 5 & $(3.20)$ & 1 & 2 & 3 & 4 & 5 \\
\hline
\end{tabular}

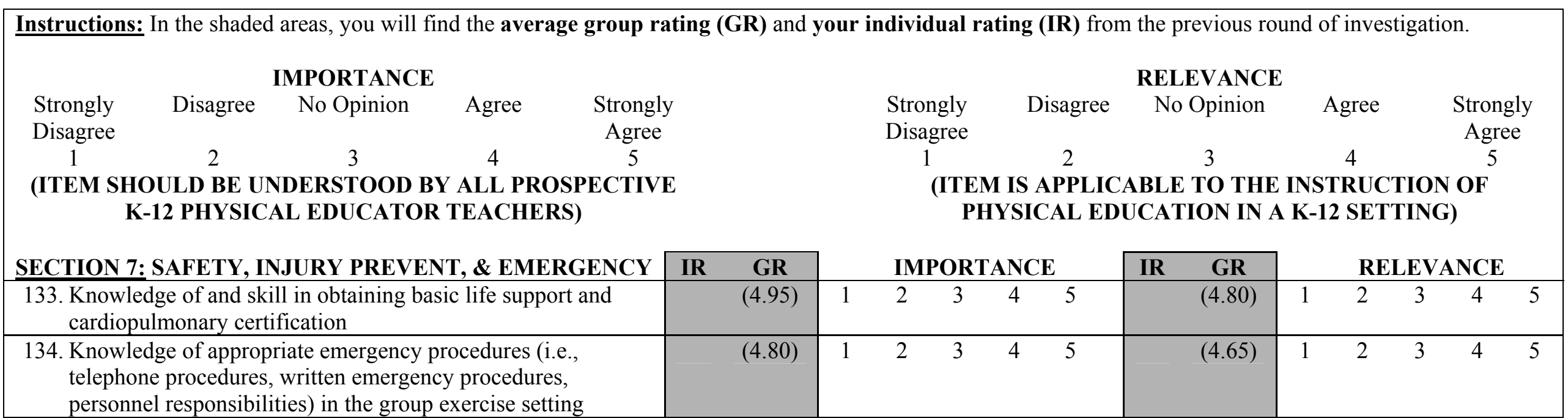




\begin{tabular}{|c|c|c|c|c|c|c|c|c|c|c|c|c|}
\hline $\begin{array}{l}\text { 135. Knowledge of basic first aid procedures for exercise-related } \\
\text { injuries, such as bleeding, strains/sprains, fractures, and } \\
\text { exercise intolerance (dizziness, syncope, heat injury) }\end{array}$ & $(4.70)$ & 1 & 2 & 3 & 4 & 5 & $(4.65)$ & 1 & 2 & 3 & $\overline{4}$ & 5 \\
\hline $\begin{array}{l}\text { 136. Knowledge of basic precautions taken in a group exercise } \\
\text { setting to ensure participant safety }\end{array}$ & $(4.60)$ & 1 & 2 & 3 & 4 & 5 & $(4.55)$ & 1 & 2 & 3 & 4 & 5 \\
\hline $\begin{array}{l}\text { 137. Ability to identify the physical and physiological signs and } \\
\text { symptoms of overtraining }\end{array}$ & $(4.10)$ & 1 & 2 & 3 & 4 & 5 & $(3.55)$ & 1 & 2 & 3 & 4 & 5 \\
\hline $\begin{array}{l}\text { 138. Ability to list the effects of temperature, humidity, altitude, } \\
\text { and pollution on physiological response to exercise }\end{array}$ & $(4.45)$ & 1 & 2 & 3 & 4 & 5 & $(4.15)$ & 1 & 2 & 3 & 4 & 5 \\
\hline $\begin{array}{l}\text { 139. Knowledge of the following terms: shin splints, sprain, strain, } \\
\text { tennis elbow, bursitis, stress fracture, tendonitis, patellar } \\
\text { femoral pain syndrome, low back pain, plantar fasciitis, and } \\
\text { rotator cuff tendonitis }\end{array}$ & $(4.20)$ & 1 & 2 & 3 & 4 & 5 & $(3.65)$ & 1 & 2 & 3 & 4 & 5 \\
\hline $\begin{array}{l}\text { 140. Skill to demonstrate exercises used for people with low back } \\
\text { pain }\end{array}$ & $(3.95)$ & 1 & 2 & 3 & 4 & 5 & $(3.40)$ & 1 & 2 & 3 & 4 & 5 \\
\hline $\begin{array}{l}\text { 141. Knowledge of hypothetical concerns and potential risks that } \\
\text { may be associated with the use of exercises such as straight } \\
\text { leg sit-ups, double leg raises, full squats, hurdlers stretch, } \\
\text { yoga plough, forceful back hyperextension, and standing bent- } \\
\text { over toe touch }\end{array}$ & $(4.60)$ & 1 & 2 & 3 & 4 & 5 & $(4.45)$ & 1 & 2 & 3 & 4 & 5 \\
\hline $\begin{array}{l}\text { 142. Skill in demonstrating appropriate emergency procedures } \\
\text { during exercise testing and/or training }\end{array}$ & $(4.11)$ & 1 & 2 & 3 & 4 & 5 & $(3.58)$ & 1 & 2 & 3 & 4 & 5 \\
\hline $\begin{array}{l}\text { 143. Knowledge of safety plans, emergency procedures, and first } \\
\text { aid techniques needed during fitness evaluations, exercise } \\
\text { testing, and exercise training }\end{array}$ & $(4.25)$ & 1 & 2 & 3 & 4 & 5 & $(3.95)$ & 1 & 2 & 3 & 4 & 5 \\
\hline $\begin{array}{l}\text { 144. Ability to identify the components that contribute to the } \\
\text { maintenance of a safe environment }\end{array}$ & $(4.37)$ & 1 & 2 & 3 & 4 & 5 & $(4.11)$ & 1 & 2 & 3 & 4 & 5 \\
\hline $\begin{array}{l}\text { 145. Knowledge of the health/fitness instructor's responsibilities, } \\
\text { limitations, and the legal implications of carrying out } \\
\text { emergency procedures }\end{array}$ & $(4.20)$ & 1 & 2 & 3 & 4 & 5 & $(4.10)$ & 1 & 2 & 3 & 4 & 5 \\
\hline $\begin{array}{l}\text { 146. Ability to describe potential musculoskeletal injuries (e.g., } \\
\text { contusions, sprains, strains, fractures), } \\
\text { cardiovascular/pulmonary complications (e.g., tachycardia, } \\
\text { bradycardia, hypotension/hypertension, tachypnea), and } \\
\text { metabolic abnormalities (e.g., fainting/syncope, } \\
\text { hypoglycemia/hyperglycemia, hypothermia/hyperthermia) }\end{array}$ & $(3.55)$ & 1 & 2 & 3 & 4 & 5 & $(3.30)$ & 1 & 2 & 3 & 4 & 5 \\
\hline
\end{tabular}


147. Knowledge of the initial management and first aid techniques associated with open wounds, musculoskeletal injuries, cardiovascular/pulmonary complications, and metabolic disorders

148. Knowledge of the components of an equipment maintenance/repair program and how it may be used to evaluate the condition of exercise equipment to reduce potential risk of injury

\begin{tabular}{|c|c|c|c|c|c|c|c|c|c|c|c|}
\hline$\overline{(4.45)}$ & 1 & 2 & 3 & 4 & 3 & $(4.25)$ & 1 & 2 & & $\overline{4}$ & 5 \\
\hline$(3.65)$ & 1 & 2 & 3 & 4 & 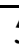 & $(3.25)$ & 1 & 2 & & 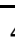 & 5 \\
\hline
\end{tabular}

Instructions: In the shaded areas, you will find the average group rating (GR) and your individual rating (IR) from the previous round of investigation.

\begin{tabular}{|c|c|c|c|c|c|c|c|c|c|}
\hline \multicolumn{5}{|c|}{ IMPORTANCE } & \multicolumn{5}{|c|}{ RELEVANCE } \\
\hline Strongly & Disagree & No Opinion & Agree & Strongly & Strongly & Disagree & No Opinion & Agree & Strongly \\
\hline Disagree & & & & Agree & Disagree & & & & Agree \\
\hline 1 & 2 & 3 & 4 & 5 & 1 & 2 & 3 & 4 & 5 \\
\hline (ITEM SI & $\begin{array}{l}\text { ULD BE U } \\
12 \text { PHYSIC }\end{array}$ & $\begin{array}{l}\text { DERSTOOD B } \\
\text { L EDUCATOF }\end{array}$ & $\begin{array}{l}\text { ALL P } \\
\text { EACH }\end{array}$ & ECTIVE & & $\begin{array}{l}\text { S APPLIC } \\
\text { ICAL ED }\end{array}$ & $\begin{array}{l}\text { BLE TO THE } \\
\text { CATION IN }\end{array}$ & $\begin{array}{l}\text { STRUC } \\
\text {-12 SET }\end{array}$ & OF \\
\hline
\end{tabular}

\section{SECTION 8: EXERCISE PROGRAMMING}

149. Knowledge of the recommended intensity, duration, frequency, and type of physical activity necessary for development of cardiorespiratory fitness in an apparently healthy population

150. Ability to differentiate between the amount of physical activity required for health benefits and the amount of exercise required for fitness development

151. Ability to describe exercises designed to enhance muscular strength and/or endurance of specific major muscle groups

152. Knowledge of the principles of overload, specificity, and progression and how they relate to exercise programming

153. Skill to teach and demonstrate appropriate exercises used in the warm-up and cool-down of a variety of group exercise classes

154. Ability to teach the components of an exercise session (i.e., warm-up, aerobic stimulus phase, cool-down, muscular strength/endurance, flexibility)

\begin{tabular}{|c|c|c|c|c|c|c|c|c|c|c|c|}
\hline IR $\quad$ GR & \multicolumn{5}{|c|}{ IMPORTANCE } & IR $\quad$ GR & \multicolumn{5}{|c|}{ RELEVANCE } \\
\hline$(4.80)$ & 1 & 2 & 3 & 4 & 5 & $(4.84)$ & 1 & 2 & 3 & 4 & 5 \\
\hline$(4.55)$ & 1 & 2 & 3 & 4 & 5 & $(4.40)$ & 1 & 2 & . & 4 & 5 \\
\hline$(4.75)$ & 1 & 2 & 3 & 4 & 5 & $(4.70)$ & 1 & 2 & . & 4 & 5 \\
\hline$(4.75)$ & 1 & 2 & 3 & 4 & 5 & $(4.60)$ & 1 & 2 & . & 4 & 5 \\
\hline$(4.45)$ & 1 & 2 & 3 & 4 & 5 & $(4.50)$ & 1 & 2 & . & 4 & 5 \\
\hline$(4.50)$ & 1 & 2 & 3 & 4 & 5 & $(4.40)$ & 1 & 2 & . & 4 & 5 \\
\hline
\end{tabular}




\begin{tabular}{|c|c|c|c|c|c|c|c|c|c|c|c|c|}
\hline $\begin{array}{l}\text { 155. Knowledge of the following terms: progressive resistance, } \\
\text { isotonic/isometric, concentric, eccentric, atrophy, } \\
\text { hypertrophy, sets, repetitions, plyometrics, Valsalva maneuver }\end{array}$ & $(4.40)$ & 1 & 2 & 3 & 4 & 5 & $(4.05)$ & 1 & 2 & 3 & 4 & 5 \\
\hline $\begin{array}{l}\text { 156. Skill to teach class participants how to monitor intensity of } \\
\text { exercise using heart rate and rating of perceived exertion } \\
\text { (RPE) }\end{array}$ & $(4.45)$ & 1 & 2 & 3 & 4 & 5 & $(4.45)$ & 1 & 2 & 3 & 4 & 5 \\
\hline $\begin{array}{l}\text { 157. Skill to teach participants how to use RPE and heart rate to } \\
\text { adjust the intensity of the exercise session }\end{array}$ & $(4.45)$ & 1 & 2 & 3 & 4 & 5 & $(4.45)$ & 1 & 2 & 3 & 4 & 5 \\
\hline $\begin{array}{l}\text { 158. Ability to calculate training heart rates using two methods: } \\
\text { percent of age-predicted maximum heart rate and heart rate } \\
\text { reserve (Karvonen) }\end{array}$ & $(4.25)$ & 1 & 2 & 3 & 4 & 5 & $(4.00)$ & 1 & 2 & 3 & 4 & 5 \\
\hline $\begin{array}{l}\text { 159. Skill to teach and demonstrate appropriate modifications in } \\
\text { specific exercises for the following groups: older adults, } \\
\text { pregnant and postnatal women, obese persons, and persons } \\
\text { with low back pain }\end{array}$ & $(3.45)$ & 1 & 2 & 3 & 4 & 5 & $(2.70)$ & 1 & 2 & 3 & 4 & 5 \\
\hline $\begin{array}{l}\text { 160. Ability to recognize proper and improper techniques in the } \\
\text { use of resistive equipment such a stability balls, weights, } \\
\text { bands, resistance bars, and water exercise equipment }\end{array}$ & $(4.40)$ & 1 & 2 & 3 & 4 & 5 & $(4.40)$ & 1 & 2 & 3 & 4 & 5 \\
\hline $\begin{array}{l}\text { 161. Ability to recognize proper and improper technique in the use } \\
\text { of cardiovascular conditioning equipment (e.g., steps, cycles, } \\
\text { slides) }\end{array}$ & $(4.30)$ & 1 & 2 & 3 & 4 & 5 & $(4.35)$ & 1 & 2 & 3 & 4 & 5 \\
\hline $\begin{array}{l}\text { 162. Skill to teach and demonstrate appropriate exercises for } \\
\text { improving range of motion of all major joints }\end{array}$ & $(4.40)$ & 1 & 2 & 3 & 4 & 5 & $(4.35)$ & 1 & 2 & 3 & 4 & 5 \\
\hline $\begin{array}{l}\text { 163. Ability to modify exercises in the group setting for apparently } \\
\text { healthy persons of various fitness levels }\end{array}$ & $(4.35)$ & 1 & 2 & 3 & 4 & 5 & $(4.35)$ & 1 & 2 & 3 & $\overline{4}$ & 5 \\
\hline $\begin{array}{l}\text { 164. Ability to teach a progression of exercises for all major } \\
\text { muscle groups to improve muscular strength and endurance }\end{array}$ & $(4.55)$ & 1 & 2 & 3 & 4 & 5 & $(4.55)$ & 1 & 2 & 3 & 4 & 5 \\
\hline $\begin{array}{l}\text { 165. Knowledge to describe the various types of interval, } \\
\text { continuous, and circuit training programs }\end{array}$ & $(4.50)$ & 1 & 2 & 3 & 4 & 5 & $(4.45)$ & 1 & 2 & 3 & 4 & 5 \\
\hline $\begin{array}{l}\text { 166. Knowledge to describe various ways a leader can take a } \\
\text { position relative to the group to enhance visibility, participant } \\
\text { interactions, and communication }\end{array}$ & $(4.05)$ & 1 & 2 & 3 & 4 & 5 & $(3.75)$ & 1 & 2 & 3 & 4 & 5 \\
\hline $\begin{array}{l}\text { 167. Ability to communicate effectively with exercise participants } \\
\text { in the group exercise session }\end{array}$ & $(4.55)$ & 1 & 2 & 3 & 4 & 5 & $(4.60)$ & 1 & 2 & 3 & 4 & 5 \\
\hline $\begin{array}{l}\text { 168. Knowledge to describe partner resistance exercises that can be } \\
\text { used in a group class setting }\end{array}$ & $(4.15)$ & 1 & 2 & 3 & 4 & 5 & $(4.10)$ & 1 & 2 & 3 & $\overline{4}$ & 5 \\
\hline
\end{tabular}




\begin{tabular}{|c|c|c|c|c|c|c|c|c|c|c|c|c|}
\hline $\begin{array}{l}\text { 169. Ability to demonstrate techniques for accommodating various } \\
\text { fitness levels within the same class }\end{array}$ & $(4.40)$ & 1 & 2 & 3 & 4 & 5 & $(4.50)$ & 1 & 2 & 3 & 4 & 5 \\
\hline $\begin{array}{l}\text { 170. Knowledge of the properties of water that affect the design of } \\
\text { a water exercise session }\end{array}$ & $(3.45)$ & 1 & 2 & 3 & 4 & 5 & $(2.95)$ & 1 & 2 & 3 & 4 & 5 \\
\hline $\begin{array}{l}\text { 171. Knowledge of basic music fundamentals, including downbeat, } \\
8 \text { count, and } 32 \text { count }\end{array}$ & $(3.10)$ & 1 & 2 & 3 & 4 & 5 & $(3.00)$ & 1 & 2 & 3 & 4 & 5 \\
\hline $\begin{array}{l}\text { 172. Skill to effectively use verbal and nonverbal cues in the group } \\
\text { exercise setting, including anticipatory, motivational, safety, } \\
\text { and educational }\end{array}$ & $(3.90)$ & 1 & 2 & 3 & 4 & 5 & $(4.00)$ & 1 & 2 & 3 & 4 & 5 \\
\hline $\begin{array}{l}\text { 173. Skill to demonstrate the proper form, alignment, and } \\
\text { technique in typical exercises used in warm-up, stimulus, } \\
\text { muscle conditioning, and cool-down phases of the group } \\
\text { session }\end{array}$ & $(4.35)$ & 1 & 2 & 3 & 4 & 5 & (4.35) & 1 & 2 & 3 & 4 & 5 \\
\hline $\begin{array}{l}\text { 174. Ability to evaluate specific exercises in terms of safety and } \\
\text { effectiveness for various participants }\end{array}$ & $(4.35)$ & 1 & 2 & 3 & 4 & 5 & $(4.40)$ & 1 & 2 & 3 & 4 & 5 \\
\hline $\begin{array}{l}\text { 175. Ability to demonstrate a familiarity with a variety of group } \\
\text { exercise formats (e.g., traditional, step, slide, muscle } \\
\text { conditioning, flexibility, indoor cycling, water fitness, } \\
\text { walking) }\end{array}$ & $(4.00)$ & 1 & 2 & 3 & 4 & 5 & $(4.00)$ & 1 & 2 & 3 & 4 & 5 \\
\hline $\begin{array}{l}\text { 176. Knowledge, skills, and abilities to prescribe and administer } \\
\text { exercise programs for apparently healthy individuals, } \\
\text { individuals at higher risk, and individuals with known disease }\end{array}$ & $(3.55)$ & 1 & 2 & 3 & 4 & 5 & $(3.30)$ & 1 & 2 & 3 & 4 & 5 \\
\hline $\begin{array}{l}\text { 177. Ability to design, implement, and evaluate individualized and } \\
\text { group exercise programs based on health history and physical } \\
\text { fitness assessments }\end{array}$ & $(3.95)$ & 1 & 2 & 3 & 4 & 5 & $(3.42)$ & 1 & 2 & 3 & 4 & 5 \\
\hline $\begin{array}{l}\text { 178. Ability to modify exercises based on age and physical } \\
\text { condition }\end{array}$ & $(4.30)$ & 1 & 2 & 3 & 4 & 5 & $(4.05)$ & 1 & 2 & 3 & 4 & 5 \\
\hline $\begin{array}{l}\text { 179. Knowledge, skills, and abilities to calculate energy cost, VO2, } \\
\text { METs, and target heart rates and apply the information to an } \\
\text { exercise prescription }\end{array}$ & $(3.95)$ & 1 & 2 & 3 & 4 & 5 & $(3.40)$ & 1 & 2 & 3 & 4 & 5 \\
\hline $\begin{array}{l}\text { 180. Ability to convert weights from pounds (lb) to kilograms }(\mathrm{kg}) \\
\text { and speed from miles per hour (MPH) to meters per minute } \\
(\mathrm{m} \cdot \mathrm{min}-1)\end{array}$ & $(3.55)$ & 1 & 2 & 3 & 4 & 5 & $(3.16)$ & 1 & 2 & 3 & 4 & 5 \\
\hline $\begin{array}{l}\text { 181. Ability to convert METs to VO2 expressed as } \mathrm{mL} \cdot \mathrm{kg}-1 \cdot \mathrm{min}-1 \text {, } \\
\mathrm{L} \cdot \mathrm{min}-1 \text {, and/or } \mathrm{mL} \cdot \mathrm{kg} \text { FFW- } 1 \cdot \mathrm{min}-1\end{array}$ & $(3.20)$ & 1 & 2 & 3 & 4 & 5 & $(2.25)$ & 1 & 2 & 3 & 4 & 5 \\
\hline
\end{tabular}




\begin{tabular}{|c|c|c|c|c|c|c|c|c|c|c|c|c|}
\hline $\begin{array}{l}\text { 182. Ability to calculate the energy cost in METs and kilocalories } \\
\text { for given exercise intensities in stepping exercise, cycle } \\
\text { ergometry, and during horizontal and graded walking and } \\
\text { running }\end{array}$ & $(3.50)$ & 1 & 2 & 3 & 4 & 5 & $(2.70)$ & 1 & 2 & 3 & 4 & 5 \\
\hline $\begin{array}{l}\text { 183. Knowledge of the approximate METs for various sport, } \\
\text { recreational, and work tasks }\end{array}$ & $(3.80)$ & 1 & 2 & 3 & 4 & 5 & $(3.30)$ & 1 & 2 & 3 & 4 & 5 \\
\hline $\begin{array}{l}\text { 184. Ability to prescribe exercise intensity based on } \mathrm{VO} 2 \text { data for } \\
\text { different modes of exercise, including graded and horizontal } \\
\text { running and walking, cycling, and stepping exercise }\end{array}$ & $(3.60)$ & 1 & 2 & 3 & 4 & 5 & $(2.95)$ & 1 & 2 & 3 & 4 & 5 \\
\hline $\begin{array}{l}\text { 185. Ability to explain and implement exercise prescription } \\
\text { guidelines for apparently health clients, increased risk clients, } \\
\text { and clients with controlled disease }\end{array}$ & $(3.30)$ & 1 & 2 & 3 & 4 & 5 & $(3.85)$ & 1 & 2 & 3 & 4 & 5 \\
\hline $\begin{array}{l}\text { 186. Ability to adapt frequency, intensity, duration, mode, } \\
\text { progression, level of supervision, and monitoring techniques } \\
\text { in exercise programs for patients with controlled chronic } \\
\text { disease (heart disease, diabetes mellitus, obesity, } \\
\text { hypertension), musculoskeletal problems, pregnancy and/or } \\
\text { postpartum and exercise-induced asthma }\end{array}$ & $(3.10)$ & 1 & 2 & 3 & 4 & 5 & $(2.80)$ & 1 & 2 & 3 & 4 & 5 \\
\hline $\begin{array}{l}\text { 187. Ability to understand the components incorporated into an } \\
\text { exercise session and the proper sequence (i.e., preexercise } \\
\text { evaluation, warm-up, aerobic stimulus phase, cool-down, } \\
\text { muscular strength and/or endurance, and flexibility) }\end{array}$ & $(4.45)$ & 1 & 2 & 3 & 4 & 5 & $(4.40)$ & 1 & 2 & 3 & 4 & 5 \\
\hline $\begin{array}{l}\text { 188. Skill in the use of various methods for establishing and } \\
\text { monitoring levels of exercise intensity, including heart rate, } \\
\text { RPE, and METs }\end{array}$ & $(4.15)$ & 1 & 2 & 3 & 4 & 5 & $(3.90)$ & 1 & 2 & 3 & 4 & 5 \\
\hline $\begin{array}{l}\text { 189. Knowledge of special precautions and modifications of } \\
\text { exercise programming for participation at altitude, different } \\
\text { ambient temperatures, humidity, and environmental pollution }\end{array}$ & $(3.95)$ & 1 & 2 & 3 & 4 & 5 & $(3.80)$ & 1 & 2 & 3 & 4 & 5 \\
\hline $\begin{array}{l}\text { 190. Ability to design resistive exercise programs to increase or } \\
\text { maintain muscular strength and/or endurance }\end{array}$ & $(4.55)$ & 1 & 2 & 3 & 4 & 5 & $(4.50)$ & 1 & 2 & 3 & 4 & 5 \\
\hline $\begin{array}{l}\text { 191. Ability to evaluate flexibility and prescribe appropriate } \\
\text { flexibility exercises for all major muscle groups }\end{array}$ & $(4.55)$ & 1 & 2 & 3 & 4 & 5 & $(4.50)$ & 1 & 2 & 3 & 4 & 5 \\
\hline $\begin{array}{l}\text { 192. Knowledge of the importance of recording exercise sessions } \\
\text { and performing periodic evaluations to assess changes in } \\
\text { fitness status }\end{array}$ & $(4.10)$ & 1 & 2 & 3 & 4 & 5 & $(4.15)$ & 1 & 2 & 3 & 4 & 5 \\
\hline
\end{tabular}




\begin{tabular}{|c|c|c|c|c|c|c|c|c|c|c|c|c|}
\hline $\begin{array}{l}\text { 193. Knowledge of the advantages and disadvantages of } \\
\text { implementation of interval, continuous, and circuit training } \\
\text { programs }\end{array}$ & $(4.30)$ & 1 & 2 & 3 & 4 & 5 & $(3.95)$ & 1 & 2 & 3 & 4 & 5 \\
\hline $\begin{array}{l}\text { 194. Ability to design training programs using interval, continuous, } \\
\text { and circuit training programs }\end{array}$ & $(4.35)$ & 1 & 2 & 3 & 4 & 5 & $(4.20)$ & 1 & 2 & 3 & 4 & 5 \\
\hline $\begin{array}{l}\text { 195. Ability to discuss the advantages and disadvantages of various } \\
\text { commercial exercise equipment in developing } \\
\text { cardiorespiratory fitness, muscular strength, and muscular } \\
\text { endurance }\end{array}$ & $(3.80)$ & 1 & 2 & 3 & 4 & 5 & $(3.45)$ & 1 & 2 & 3 & 4 & 5 \\
\hline $\begin{array}{l}\text { 196. Knowledge of the types of exercise programs available in the } \\
\text { community and how these programs are appropriate for } \\
\text { various populations }\end{array}$ & $(3.85)$ & 1 & 2 & 3 & 4 & 5 & $(3.70)$ & 1 & 2 & 3 & 4 & 5 \\
\hline $\begin{array}{l}\text { 197. Ability to include participants with different individual needs } \\
\text { into a group exercise environment }\end{array}$ & $(4.25)$ & 1 & 2 & 3 & 4 & 5 & $(4.30)$ & 1 & 2 & 3 & 4 & 5 \\
\hline $\begin{array}{l}\text { 198. Knowledge of the concept of periodization as it relates to } \\
\text { strength and endurance exercise programming }\end{array}$ & (3.89) & 1 & 2 & 3 & 4 & 5 & (3.47) & 1 & 2 & 3 & 4 & 5 \\
\hline $\begin{array}{l}\text { 199. Knowledge of the special concerns and guidelines } \\
\text { recommended for children and adolescent strength training }\end{array}$ & $(4.70)$ & 1 & 2 & 3 & 4 & 5 & $(4.70)$ & 1 & 2 & 3 & 4 & 5 \\
\hline $\begin{array}{l}\text { 200. Knowledge of the published exercise guidelines and } \\
\text { recommendations for children, adolescents, adults and older } \\
\text { adults }\end{array}$ & $(4.65)$ & 1 & 2 & 3 & 4 & 5 & $(4.55)$ & 1 & 2 & 3 & 4 & 5 \\
\hline
\end{tabular}

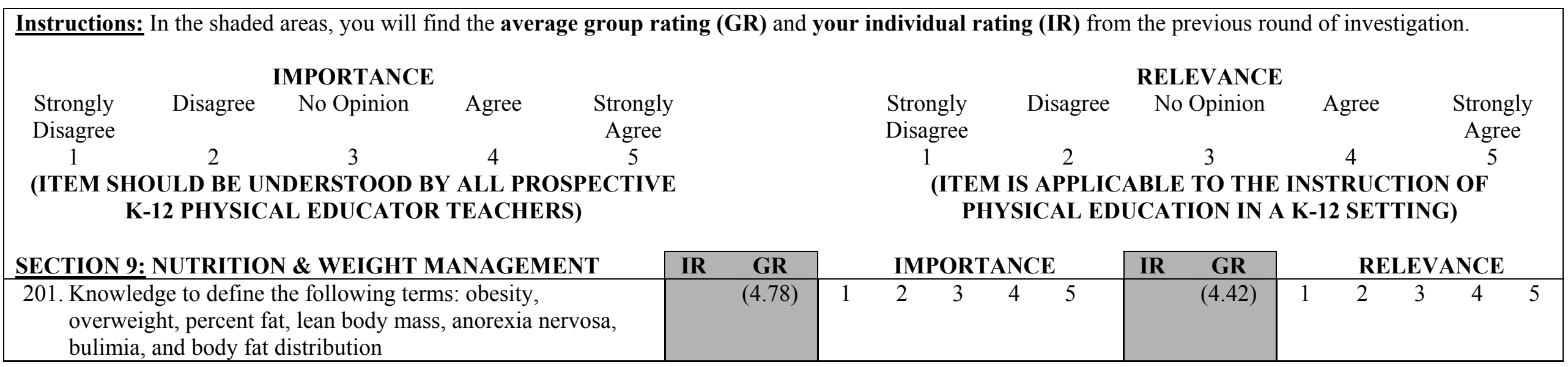




\begin{tabular}{|c|c|c|c|c|c|c|c|c|c|c|c|c|}
\hline $\begin{array}{l}\text { 202. Knowledge of the relationship between body composition and } \\
\text { health }\end{array}$ & $(4.65)$ & 1 & 2 & 3 & 4 & 5 & $(4.40)$ & 1 & 2 & 3 & 4 & 5 \\
\hline $\begin{array}{l}\text { 203. Knowledge of the effects of diet plus exercise, diet alone, and } \\
\text { exercise alone as methods for modifying body composition }\end{array}$ & $(4.60)$ & 1 & 2 & 3 & 4 & 5 & $(4.50)$ & 1 & 2 & 3 & 4 & 5 \\
\hline $\begin{array}{l}\text { 204. Knowledge of the importance of an adequate daily energy } \\
\text { intake for healthy weight management }\end{array}$ & $(4.40)$ & 1 & 2 & 3 & 4 & 5 & $(4.30)$ & 1 & 2 & 3 & 4 & 5 \\
\hline $\begin{array}{l}\text { 205. Ability to differentiate between fat soluble and water-soluble } \\
\text { vitamins }\end{array}$ & $(3.35)$ & 1 & 2 & 3 & 4 & 5 & $(2.55)$ & 1 & 2 & 3 & 4 & 5 \\
\hline $\begin{array}{l}\text { 206. Ability to describe the importance of maintaining normal } \\
\text { hydration before, during, and after exercise }\end{array}$ & $(4.75)$ & 1 & 2 & 3 & 4 & 5 & $(4.70)$ & 1 & 2 & 3 & 4 & 5 \\
\hline 207. Knowledge of the USDA Food Pyramid & $(4.30)$ & 1 & 2 & 3 & $\overline{4}$ & 5 & $(4.15)$ & 1 & 2 & 3 & 4 & 5 \\
\hline $\begin{array}{l}\text { 208. Knowledge of the importance of calcium and iron in women's } \\
\text { health }\end{array}$ & $(4.05)$ & 1 & 2 & 3 & 4 & 5 & $(3.75)$ & 1 & 2 & 3 & 4 & 5 \\
\hline $\begin{array}{l}\text { 209. Ability to describe the myths and consequences associated } \\
\text { with inappropriate weight loss methods (e.g., saunas, } \\
\text { vibrating belts, body wraps, electric simulators, sweat suits, } \\
\text { fad diets) }\end{array}$ & $(4.45)$ & 1 & 2 & 3 & 4 & 5 & $(4.20)$ & 1 & 2 & 3 & 4 & 5 \\
\hline $\begin{array}{l}\text { 210. Knowledge of the number of kilocalories in one gram of } \\
\text { carbohydrate, fat, protein, and alcohol }\end{array}$ & $(3.90)$ & 1 & 2 & 3 & 4 & 5 & $(3.20)$ & 1 & 2 & 3 & 4 & 5 \\
\hline $\begin{array}{l}\text { 211. Knowledge of the number of kilocalories equivalent to losing } \\
1 \text { pound of body fat }\end{array}$ & $(4.00)$ & 1 & 2 & 3 & 4 & 5 & $(3.55)$ & 1 & 2 & 3 & 4 & 5 \\
\hline $\begin{array}{l}\text { 212. Knowledge, skills, and abilities to provide information } \\
\text { concerning nutrition and the role of diet and exercise on body } \\
\text { composition and weight control }\end{array}$ & $(4.50)$ & 1 & 2 & 3 & 4 & 5 & $(4.45)$ & 1 & 2 & 3 & 4 & 5 \\
\hline $\begin{array}{l}\text { 213. Ability to describe the health implications of variation in body } \\
\text { fat distribution patterns and the significance of waist to hip } \\
\text { ratio }\end{array}$ & $(4.00)$ & 1 & 2 & 3 & 4 & 5 & $(3.80)$ & 1 & 2 & 3 & 4 & 5 \\
\hline $\begin{array}{l}\text { 214. Knowledge of the guidelines for caloric intake for an } \\
\text { individual desiring to lose or gain weight }\end{array}$ & $(4.25)$ & 1 & 2 & 3 & 4 & 5 & $(4.25)$ & 1 & 2 & 3 & 4 & 5 \\
\hline $\begin{array}{l}\text { 215. Knowledge of common nutritional ergogenic aids, the } \\
\text { purported mechanism of action, and any risk and/or benefit } \\
\text { (e.g., carbohydrates, protein/amino acids, vitamins, minerals, } \\
\text { sodium bicarbonate, creatine, bee pollen) }\end{array}$ & $(3.90)$ & 1 & 2 & 3 & 4 & 5 & $(3.45)$ & 1 & 2 & 3 & 4 & 5 \\
\hline
\end{tabular}




\begin{tabular}{|c|c|c|c|c|c|c|c|c|c|c|c|c|}
\hline $\begin{array}{l}\text { 216. Knowledge of nutritional factors related to the female athlete } \\
\text { triad syndrome (i.e., eating disorders, menstrual cycle } \\
\text { abnormalities, and osteoporosis) }\end{array}$ & $(3.95)$ & 1 & 2 & 3 & 4 & 5 & $(3.58)$ & 1 & 2 & 3 & 4 & 5 \\
\hline $\begin{array}{l}\text { 217. Knowledge of the NIH Consensus statement regarding health } \\
\text { risks of obesity, Nutrition for Physical Fitness Position Paper } \\
\text { of the American Dietetic Association, and the ACSM Position } \\
\text { Stand on proper and improper weight loss programs }\end{array}$ & $(4.16)$ & 1 & 2 & 3 & 4 & 5 & $(3.79)$ & 1 & 2 & 3 & 4 & 5 \\
\hline 218. Knowledge of NCEP II guidelines for lipid management & $(3.42)$ & 1 & 2 & 3 & 4 & 5 & $(3.11)$ & 1 & 2 & 3 & 4 & 5 \\
\hline
\end{tabular}

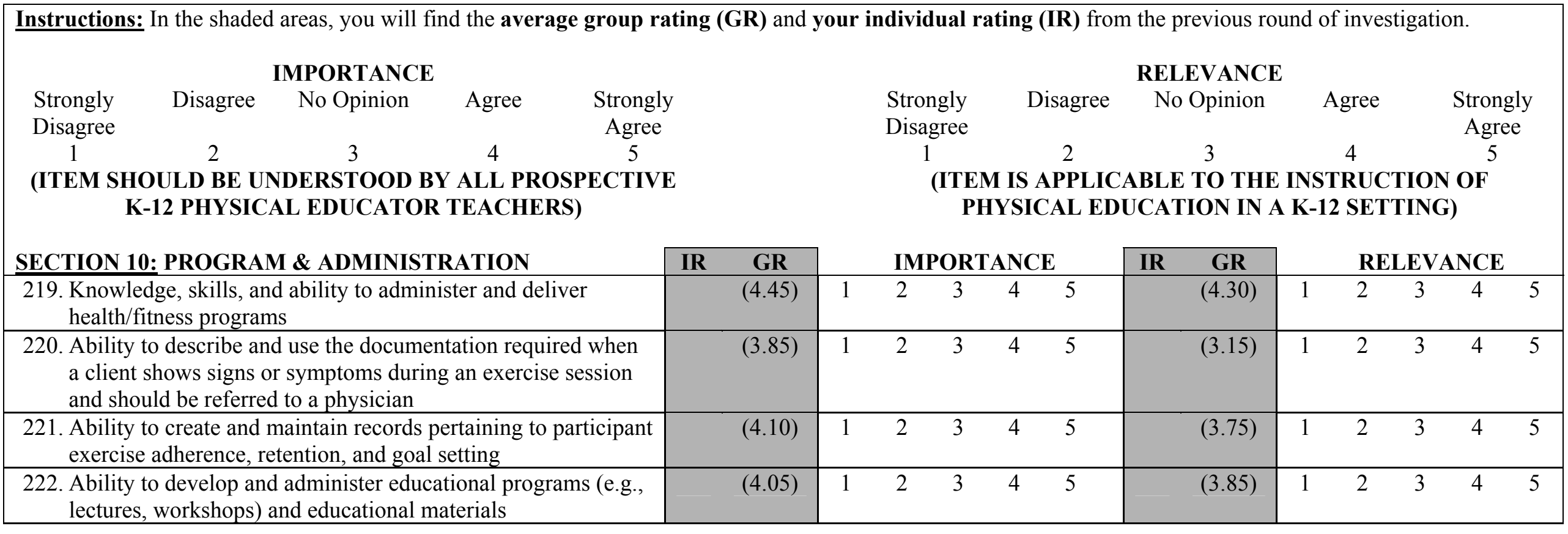




\section{Section 11: Recommended Instructional Methods}

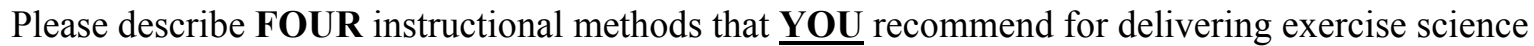
content to prospective K-12 physical education teachers within the physical education teacher education curriculum. In addition, please provide an explanation of WHY you feel that each of these instructional methods is effective.

My FOUR recommended instructional method for delivering exercise science content to prospective physical educators are:

\section{METHOD 1:}

Why did you recommend this instructional method?

\section{METHOD 2:}

Why did you recommend this instructional method? 
METHOD 3:

Why did you recommend this instructional method?

\section{METHOD 4:}

Why did you recommend this instructional method?

THANK YOU AGAIN FOR YOUR HELP IN COMPLETING THIS PROJECT! 


\section{APPENDIX D}

Modified Delphi Investigation of Exercise Science in Physical Education Teacher Education Sample Course Syllabus 


\section{West Virginia University \\ School of Physical Education \\ PET 198K Teaching of Fitness Education}

$\begin{array}{llll}\text { Instructor } & \text { Sean M. Bulger } & \text { Office Location } & \text { EMH Room 13 } \\ \text { E-mail } & \text { sbulger@wvu.edu } & \text { Office Hours } & \text { M/W 9:00-12:00 PM } \\ \text { Phone } & \text { (304) 293-3295 ext. 5250 } & \text { Lecture } & \text { M/W 3:00-3:50 PM (ALH 711) }\end{array}$

Course 1. AAHPERD (1999). Physical Education for Lifelong Fitness: The Physical Best

Texts Teacher's Guide. Champaign, IL: Human Kinetics.

2. AAHPERD (1999). Physical Best Activity Guide: Elementary Level. Champaign, IL: Human Kinetics.

3. AAHPERD (1999). Physical Best Activity Guide: Secondary Level. Champaign, IL: Human Kinetics.

4. Reserve Readings will be available through electronic reserve.

Course This course introduces prospective physical education teachers to the foundations and Goals components of health-related fitness as well as appropriate curriculum for K-12, effective teaching principles, and the assessment of health-related fitness. Students become eligible for Physical Best Health/Fitness Specialist Certification.

Course At the completion of this course, the student will be able to demonstrate an Objectives understanding of the following areas:

1. The historical relationships among physical education, physical activity, and healthrelated fitness. Student performance will be assessed through the completion of daily lecture reactions, study guide questions, quizzes, and a final exam.

2. The basic training principles of physical conditioning and the implementation of developmentally appropriate training protocol in children's physical activity program design. Student performance will be assessed through the completion of daily lecture reactions, study guide questions, quizzes, and a final exam.

3. The components of health-related fitness in school-aged children, including cardiovascular endurance, muscular strength and endurance, flexibility, and body composition. Student performance will be assessed through the completion of daily lecture reactions, study guide questions, quizzes, and a final exam.

4. The various training methods and their immediate and long-term effects on the growth and maturation of children. Student performance will be assessed through the completion of daily lecture reactions, study guide questions, quizzes, and a final exam.

5. The impact of maturation, aging, drugs, and nutrition on fitness performance. Student performance will be assessed through the completion of daily lecture reactions, study guide questions, quizzes, and a final exam. 
6. The development of alternative curricular and instructional strategies for the promotion of children's physical activity and health-related fitness. Student performance will be assessed through the completion of daily lecture reactions, study guide questions, quizzes, clinical lab experiences, a curriculum \& instruction project, and a final exam.

7. The assessment issues and techniques typically associated with children's physical activity and health-related fitness programming in the schools. Student performance will be assessed through the completion of daily lecture reactions, study guide questions, quizzes, clinical lab experiences, a curriculum \& instruction project, and a final exam.

8. The fitness-related safety considerations that contribute to the provision of proper instruction, adequate supervision, and a safe exercise environment for children. Student performance will be assessed through the completion of daily lecture reactions, study guide questions, quizzes, clinical lab experiences, a curriculum \& instruction project, and a final exam.

9. The requirements for the successful completion of the AAHPERD Physical Best Health/Fitness Specialist Certification. Student performance will be assessed through the completion of daily lecture reactions, study guide questions, quizzes, clinical lab experiences, a curriculum \& instruction project, and a final exam.

Course Criteria
1. Review the course schedule and familiarize yourself with the assigned readings and study guide questions prior to the start of each unit.

2. Complete the assigned course readings from the text and additional sources as indicated in the course schedule. You will be expected to read all of the assigned readings prior to your attendance at the related lecture. Failure to comply with this requirement may result in your inability to adequately complete various lecture or lab assignments.

3. Attend all lecture and lab sessions prepared to ask and answer questions regarding the previously assigned course materials.

4. Complete daily lecture reactions that require you to actively reflect on the previously assigned course materials and the day's instructional activities.

5. Complete four (4) study guides and written quizzes. Each quiz will consist of objective test items, such as multiple choice, true/false, matching, fill-in-the-blank, and short answer questions derived from readings, lectures, and class discussions.

6. Participate in eight (8) clinical lab experiences. These clinical lab experiences will include a combination of in-class and out-of-class activities.

7. Participate in one (1) curriculum and instruction project concerning the teaching of health-related fitness in a school-based physical education setting.

8. Complete a final written examination. The final exam will consist of objective test items, such as multiple choice, true/false, matching, fill-in-the-blank, and short answer questions derived from readings, lectures, and class discussions. 
Attendance Policy

Social Justice Policy

Academic Integrity/ Dishonesty Policy
Students must attend every lecture and lab unless an exceptional emergency occurs. If you know that you will be unable to attend a certain class for a legitimate reason (i.e. athletic event, funeral, etc.) it is your responsibility to notify the instructor ahead of time and make arrangements to make-up the missed educational experience. Students will not receive credit for any course work that is late or incomplete due to an unexcused absence.

West Virginia University (WVU) is committed to social justice. I concur with that commitment and expect to foster a nurturing environment based upon communication, mutual respect, and non-discrimination. Our University does not discriminate on the basis of race, sex, age, disability, veteran status, religion, sexual orientation, color or national origin. Any suggestions as to how to further such a positive and open environment in this class will be appreciated and given serious consideration.

The academic development of students and the overall integrity of the institution are primary responsibilities of WVU. Academic dishonesty is condemned at all levels of life, indicating an inability to meet and face issues and creating an atmosphere of mistrust, disrespect, and insecurity. In addition, it is essential in an academic community that grades accurately reflect the attainment of the individual student. Faculty, students, and administrators have shared responsibilities in maintaining the academic integrity essential for the University to accomplish its mission.

Students should act to prevent opportunities for academic dishonesty to occur and in such a manner to discourage any type of academic dishonesty.

Faculty members are expected to remove opportunities for cheating, whether related to test construction, test confidentiality, test administration, or test grading. This same professional care should be exercised with regard to oral and written reports, laboratory assignments, and grade books.

Deans and department chairpersons are expected to acquaint all faculty members with expected professional behavior regarding academic integrity, and to continue to remind them of their responsibility. Deans and department chairpersons shall assist faculty members and students in handling first-offense cheating allegations at the lowest possible level in the University, and with discretion to prevent damage to the reputation of any person who has not been found guilty in the prescribed manner.

Each member of the teaching faculty and all other WVU employees, including but not limited to assistants, proctors, office personnel, custodians, and public safety officers, shall promptly report each known case of academic dishonesty to the appropriate supervisor, department chairperson, or dean of the college or school concerned, and to the Office of Judicial Programs, Office of Student Life. 


\section{PET 198K Course Schedule}

\begin{tabular}{|c|c|}
\hline Monday & Wednesday \\
\hline $\begin{array}{l}\text { 1/10 Course Orientation I } \\
\square \text { Course Enrollment } \\
\square \text { HR Fitness in the PETE Curriculum } \\
\square \text { Course Syllabus (Instructor Information, } \\
\text { Texts, Goals, Objectives, \& Criteria) } \\
\square \text { Course Assessment: } \\
\text { A. Knowledge Pre-test }\end{array}$ & $\begin{array}{l}\text { 1/12 Course Orientation II } \\
\square \text { Course Syllabus (Policies, Schedule, \& } \\
\text { Grading) } \\
\square \text { Overview of Course Units 1-5: Physical } \\
\text { Activity \& Fitness Theory } \\
\text { A. Lecture Reactions } \\
\text { B. Study Guide Questions } \\
\text { C. Quizzes \& Final Examination }\end{array}$ \\
\hline $\begin{array}{c}\text { 1/17 No Class - Martin Luther King Jr.'s } \\
\text { Birthday Observed }\end{array}$ & $\begin{array}{l}\text { 1/19 Course Orientation III } \\
\square \text { Overview of Course Units 6-12: Curriculum } \\
\text { \& Instruction Project } \\
\text { A. Literature Review \& Internet Search } \\
\text { B. Instructional Material Design } \\
\text { C. Assessment Material Design } \\
\text { D. Teaching Clinics } \\
\text { E. Group Portfolio } \\
\square \text { Overview of Course Units 13-20: Clinical } \\
\text { Lab Experiences } \\
\text { A. Lab Performance Assessment Criteria }\end{array}$ \\
\hline $\begin{array}{l}\text { 1/24 Unit } 1 \text { Lecture } 1 \\
\square \quad \text { Lecture Topic: Foundations of Health- } \\
\text { Related Fitness } \\
\square \text { Assigned Readings: } \\
\text { A. AAHPERD (1999). Physical Education } \\
\quad \text { for Lifelong Fitness: The Physical Best } \\
\text { Teacher's Guide. pp. 1-83 [Text] } \\
\square \text { Course Assessment: } \\
\text { A. Lecture Reaction }\end{array}$ & $\begin{array}{l}\text { 1/26 Unit } 1 \text { Lecture } 2 \\
\square \quad \text { Lecture Topic: General Health Benefits of } \\
\text { Physical Activity } \\
\square \text { Assigned Readings: } \\
\text { A. Corbin \& Pangrazi (1999). The Health } \\
\text { Benefits of Physical Activity. pp. 43-48 } \\
\text { [Reserve] } \\
\text { B. Corbin \& Pangrazi (1999). What You } \\
\text { Need to Know About The...Surgeon } \\
\text { General's Report on Physical Activity } \\
\text { and Health. pp. 49-58 [Reserve] } \\
\square \text { Course Assessment: } \\
\text { A. Lecture Reaction }\end{array}$ \\
\hline $\begin{array}{l}\text { 1/31 Unit } 1 \text { Lecture } 3 \\
\square \text { Lecture Topic: Children's Physical Activity } \\
\square \text { Assigned Readings: } \\
\text { A. Corbin, Pangrazi, \& Welk (1999). } \\
\text { Toward an Understanding of } \\
\text { Appropriate Physical Activity Levels for } \\
\text { Youth. pp. 153-161 [Reserve] } \\
\text { B. Bar-Or (1999). Health Benefits of } \\
\text { Physical Activity during Childhood and } \\
\text { Adolescence. pp. 163-167 [Reserve] } \\
\square \text { Course Assessment: } \\
\text { A. Lecture Reaction }\end{array}$ & $\begin{array}{l}\text { 2/02 Unit } 1 \text { Quiz } \\
\square \text { Course Assessment: } \\
\text { A. Literature Search Assignment } \\
\text { B. Unit } 1 \text { Study Guide Questions } \\
\text { C. Unit } 1 \text { Quiz } \\
\text { The Unit } 1 \text { quiz will consist of various objective } \\
\text { and short answer questions that are based on } \\
\text { lectures } 1-3 \text { and the assigned course readings. } \\
\text { You will be given the entire class period to } \\
\text { complete the quiz. }\end{array}$ \\
\hline
\end{tabular}




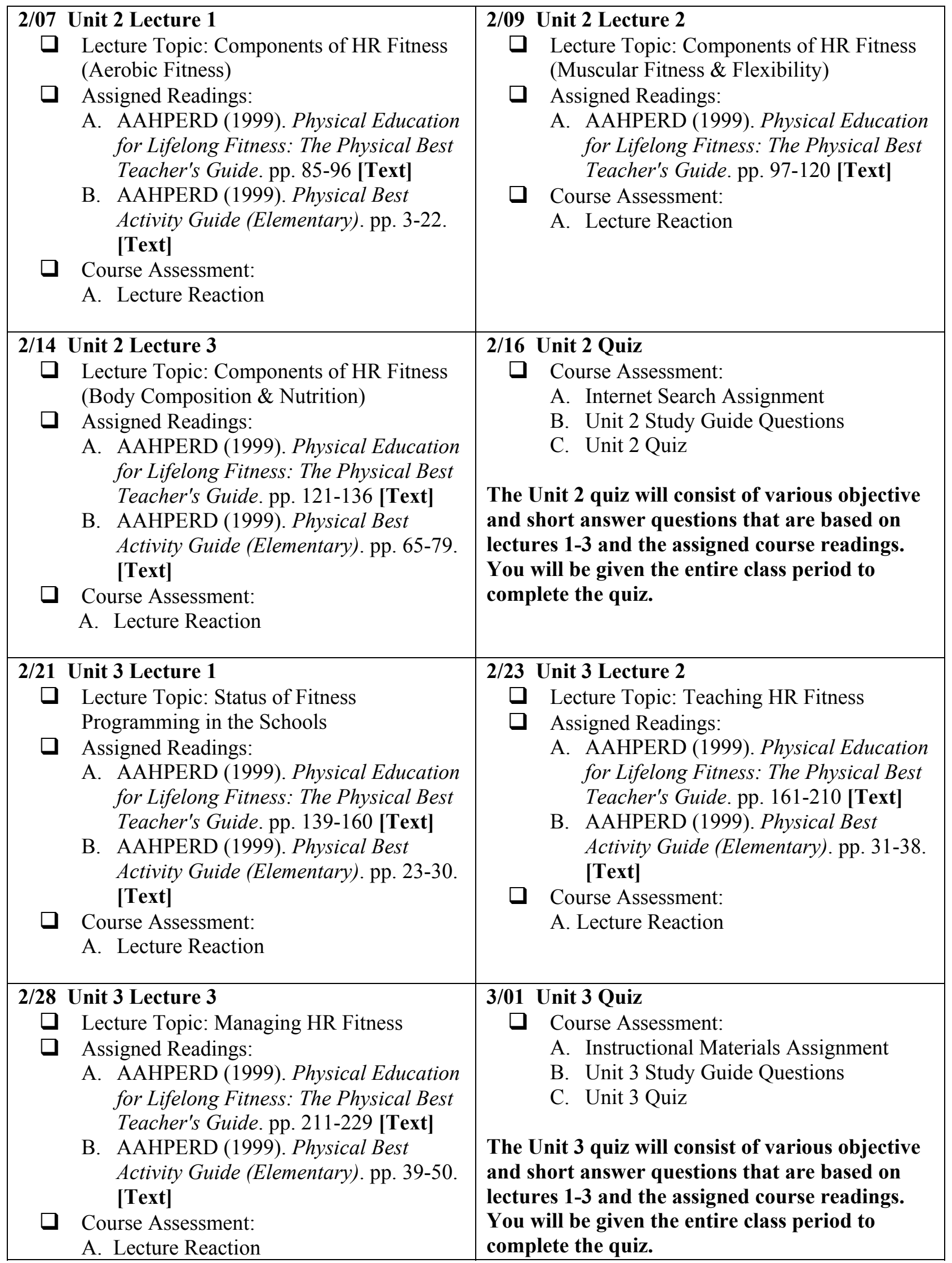




\begin{tabular}{|c|c|}
\hline $\begin{array}{l}\text { 3/06 Unit } 4 \text { Lecture } 1 \\
\square \text { Lecture Topic: Principles of Assessment } \\
\square \text { Assigned Readings: } \\
\text { A. AAHPERD (1999). Physical Education } \\
\text { for Lifelong Fitness: The Physical Best } \\
\text { Teacher's Guide. pp. 233-249 [Text] } \\
\text { B. AAHPERD (1999). Physical Best } \\
\text { Activity Guide (Elementary). pp. 51-64. } \\
\text { [Text] } \\
\square \text { Course Assessment: } \\
\text { A. Lecture Reaction }\end{array}$ & $\begin{array}{l}\text { 3/08 Unit } 4 \text { Lecture } 2 \\
\square \text { Lecture Topic: Assessing HR Physical } \\
\text { Fitness \& Knowledge } \\
\square \text { Assigned Readings: } \\
\text { A. AAHPERD (1999). Physical Education } \\
\quad \text { for Lifelong Fitness: The Physical Best } \\
\quad \text { Teacher's Guide. pp. 251-271 [Text] } \\
\square \text { Course Assessment: } \\
\text { A. Lecture Reaction }\end{array}$ \\
\hline $\begin{array}{l}\text { 3/13 Unit } 4 \text { Lecture } 3 \\
\square \quad \text { Lecture Topic: Assessing the Affective } \\
\text { Domain \& Participation in Physical Activity } \\
\square \quad \text { Assigned Readings: } \\
\text { A. AAHPERD (1999). Physical Education } \\
\quad \text { for Lifelong Fitness: The Physical Best } \\
\quad \text { Teacher's Guide. pp. 273-288 [Text] } \\
\square \text { Course Assessment: } \\
\text { A. Lecture Reaction }\end{array}$ & $\begin{array}{l}\text { 3/15 Unit } 4 \text { Quiz } \\
\text { Course Assessment: } \\
\text { A. Assessment Materials Assignment } \\
\text { B. Unit } 4 \text { Study Guide Questions } \\
\text { C. Unit } 4 \text { Quiz } \\
\text { The Unit } 4 \text { quiz will consist of various objective } \\
\text { and short answer questions that are based on } \\
\text { lectures 1-3 and the assigned course readings. } \\
\text { You will be given the entire class period to } \\
\text { complete the quiz. }\end{array}$ \\
\hline 3/20 Open Date & 3/22 Open Date \\
\hline 3/27 No Class - Spring Break & 2/29 No Class - Spring Break \\
\hline $\begin{array}{l}\text { 4/03 Lab } 1 \text { (Elementary School) } \\
\square \text { Lab Topic: Aerobic Fitness (Elementary) } \\
\square \text { Assigned Readings: } \\
\text { A. AAHPERD (1999). Physical Best } \\
\text { Activity Guide (Elementary). pp. 83- } \\
\text { 119. [Text] } \\
\square \text { Course Assessment: } \\
\text { A. Attendance } \\
\text { B. Attire } \\
\text { C. Participation } \\
\text { D. Reflection }\end{array}$ & $\begin{array}{l}\text { 4/05 Lab } 2 \text { (Elementary School) } \\
\square \text { Lab Topic: Body Composition (Elementary) } \\
\square \text { Assigned Readings: } \\
\text { A. AAHPERD (1999). Physical Best } \\
\text { Activity Guide (Elementary). pp. 199- } \\
\text { 227. [Text] } \\
\square \text { Course Assessment: } \\
\text { A. Attendance } \\
\text { B. Attire } \\
\text { C. Participation } \\
\text { D. Reflection }\end{array}$ \\
\hline
\end{tabular}




\begin{tabular}{|c|c|}
\hline $\begin{array}{l}\text { 4/10 Lab } 3 \text { (Elementary School) } \\
\square \quad \text { Lab Topic: Muscular Strength \& Endurance } \\
\text { (Elementary) } \\
\text { Assigned Readings: } \\
\text { A. AAHPERD (1999). Physical Best } \\
\text { Activity Guide (Elementary). pp. 121- } \\
\text { 157. [Text] } \\
\square \text { Course Assessment: } \\
\text { A. Attendance } \\
\text { B. Attire } \\
\text { C. Participation } \\
\text { D. Reflection }\end{array}$ & $\begin{array}{l}\text { 4/12 Lab } 4 \text { (Elementary School) } \\
\square \quad \text { Lab Topic: Flexibility (Elementary) } \\
\square \quad \text { Assigned Readings: } \\
\text { A. AAHPERD (1999). Physical Best } \\
\text { Activity Guide (Elementary). pp. 159- } \\
\text { 197. [Text] } \\
\square \text { Course Assessment: } \\
\text { A. Attendance } \\
\text { B. Attire } \\
\text { C. Participation } \\
\text { D. Reflection }\end{array}$ \\
\hline $\begin{array}{l}\text { 4/17 Lab } 5 \text { (Middle School) } \\
\square \text { Lab Topic: Aerobic Fitness \& Body } \\
\text { Composition (Middle) } \\
\text { Assigned Readings: } \\
\text { A. AAHPERD (1999). Physical Best } \\
\text { Activity Guide (Secondary). pp. 83-119 } \\
\quad \text { and 183-211. [Text] } \\
\square \text { Course Assessment: } \\
\text { A. Attendance } \\
\text { B. Attire } \\
\text { C. Participation } \\
\text { D. Reflection }\end{array}$ & $\begin{array}{l}\text { 4/19 Lab } 6 \text { (Middle School) } \\
\square \text { Lab Topic: Muscular Strength /Endurance } \\
\text { \& Flexibility (Middle) } \\
\text { Assigned Readings: } \\
\text { A. AAHPERD (1999). Physical Best } \\
\text { Activity Guide (Secondary). pp. 121- } \\
157 \text { and 159-181. [Text] } \\
\square \text { Course Assessment: } \\
\text { A. Attendance } \\
\text { B. Attire } \\
\text { C. Participation } \\
\text { D. Reflection }\end{array}$ \\
\hline $\begin{array}{l}\text { 4/24 Lab } 7 \text { (Secondary School) } \\
\square \quad \text { Lab Topic: Aerobic Fitness \& Body } \\
\text { Composition (Secondary) } \\
\square \text { Assigned Readings: } \\
\text { A. AAHPERD (1999). Physical Best } \\
\quad \text { Activity Guide (Secondary). pp. 83-119 } \\
\quad \text { and 183-211 [Text] } \\
\square \text { Course Assessment: } \\
\text { A. Attendance } \\
\text { B. Attire } \\
\text { C. Participation } \\
\text { D. Reflection }\end{array}$ & $\begin{array}{l}\text { 4/26 Lab } 8 \text { (Secondary School) } \\
\square \quad \text { Lab Topic: Muscular Strength /Endurance } \\
\text { \& Flexibility (Secondary) } \\
\text { Assigned Readings: } \\
\text { A. AAHPERD (1999). Physical Best } \\
\text { Activity Guide (Secondary). pp. 121- } \\
157 \text { and 159-181. [Text] } \\
\square \text { Course Assessment: } \\
\text { A. Attendance } \\
\text { B. Attire } \\
\text { C. Participation } \\
\text { D. Reflection }\end{array}$ \\
\hline \multicolumn{2}{|c|}{$\begin{array}{l}\text { 5/02 Final Exam Week (11-1 PM) } \\
\square \text { Course Assessment: } \\
\text { A. Take-home portion of the final exam. } \\
\text { B. The in-class portion of the final exam will consist of various objective and short answer } \\
\text { questions that are based on lectures } 1-3 \text { and the assigned course readings. You will be given } \\
\text { the entire testing period to complete the exam. }\end{array}$} \\
\hline
\end{tabular}




\section{PET 198K Course Grading Sheet}

Final course grades will be determined on a mastery system. All course assignments have been divided into twenty (20) units. The following scale will be used to determine the final course grades:

$\begin{array}{rc}20 \text { Units Passed } & \text { A } \\ 19 \text { Units Passed } & \text { B } \\ 18 \text { Units Passed } & \text { C } \\ 17 \text { Units Passed } & \text { D } \\ >16 \text { Units Passed } & \text { F }\end{array}$

I. Physical Activity \& Fitness Theory (Units 1-4)

\begin{tabular}{|l|r|r|r|r|r|r|r|}
\hline \multicolumn{1}{|c|}{ Unit Name } & $\begin{array}{c}\text { Lecture } \\
\text { React 1 }\end{array}$ & $\begin{array}{c}\text { Lecture } \\
\text { React 2 }\end{array}$ & $\begin{array}{r}\text { Lecture } \\
\text { React 3 }\end{array}$ & $\begin{array}{c}\text { Study } \\
\text { Guide }\end{array}$ & $\begin{array}{c}\text { Quiz } \\
\text { Grade }\end{array}$ & $\begin{array}{c}\text { Point } \\
\text { Total }\end{array}$ & $\begin{array}{c}\text { Units } \\
\text { Passed }\end{array}$ \\
\hline Unit 1 Foundations & $/ 5$ & $/ 5$ & $/ 5$ & $/ 5$ & $/ 80$ & $/ 100$ & \\
\hline Unit 2 HR Fitness & $/ 5$ & $/ 5$ & $/ 5$ & $/ 5$ & $/ 80$ & $/ 100$ & \\
\hline Unit 3 Curriculum & $/ 5$ & $/ 5$ & $/ 5$ & $/ 5$ & $/ 80$ & $/ 100$ & \\
\hline Unit 4 Assessment & $/ 5$ & $/ 5$ & $/ 5$ & $/ 5$ & $/ 80$ & $/ 100$ & \\
\hline
\end{tabular}

\section{Criteria for Passing Units 1-4:}

Student must obtain $\mathbf{8 0}$ out of $\mathbf{1 0 0}$ possible points in order to pass each unit.

- Student must turn in lecture reactions immediately after each lecture session.

$\square$ Student must turn in study guide questions at the start of class on the appropriate date. Study guide questions must be typed and stapled together.

$\square$ Student will be afforded the opportunity to retake two (2) quizzes during the semester. It is the student's responsibility to schedule an appointment with the course instructor for any quiz retake. No retake will be permitted if the student missed a quiz due to an unexcused absence!

$\square$ If the student fails to demonstrate competency in a unit after a quiz retake, further opportunity for remediation will be at the discretion of the instructor. A time schedule for an individualized remediation plan must be agreed upon by the instructor and student immediately following notification of the quiz retake grade. Special consideration will be given to those students who have performed satisfactorily in the other components of the unit.

\section{Quiz Retake Checklist}

\begin{tabular}{|c|c|c|}
\hline Quiz \# & / Date & Quiz Grade: $\quad / 80$ \\
\hline
\end{tabular}

*Student is allotted a total of two (2) quiz retakes for the semester. Retakes may be used at your discretion for unit quizzes 1-4 only.

\section{Physical Activity \& Fitness Theory (Unit 5)}

\begin{tabular}{|c|c|c|c|c|}
\hline Unit Name & Take-Home & In-Class & Point Total & Unit Passed \\
\hline Unit 5 Final Exam & $/ 20$ & $/ 80$ & $/ 100$ & \\
\hline
\end{tabular}

\section{Criteria for Passing Unit 5:}

$\square$ Student must obtain $\mathbf{8 0}$ out of $\mathbf{1 0 0}$ possible points in order to pass this unit. 
III. Curriculum \& Instruction Project (Units 6-12)

\begin{tabular}{|c|c|c|c|c|c|c|c|}
\hline \multirow[t]{2}{*}{ Unit Name } & \multicolumn{5}{|c|}{ Assessment Criteria } & \multirow{2}{*}{$\begin{array}{l}\text { Point } \\
\text { Total }\end{array}$} & \multirow{2}{*}{$\begin{array}{c}\text { Units } \\
\text { Passed }\end{array}$} \\
\hline & 1 & 2 & 3 & 4 & 5 & & \\
\hline Unit 6 Literature Review & $/ 4$ & 14 & 14 & $/ 4$ & 14 & 120 & \\
\hline Unit 7 Internet Search & $/ 4$ & $/ 4$ & $/ 4$ & $/ 4$ & $/ 4$ & $/ 20$ & \\
\hline Unit 8 Instructional Materials & 14 & $/ 4$ & 14 & 14 & 14 & 120 & \\
\hline Unit 9 Assessment Materials & 14 & 14 & $/ 4$ & 14 & 14 & 120 & \\
\hline Unit 10 Teaching (Clinic 1) & $/ 4$ & 14 & 14 & 14 & 14 & $/ 20$ & \\
\hline Unit 11 Teaching (Clinic 2) & 14 & 14 & 14 & 14 & 14 & 120 & \\
\hline Unit 12 Instructional Portfolio & $/ 4$ & $/ 4$ & $/ 4$ & $/ 4$ & $/ 4$ & $/ 20$ & \\
\hline
\end{tabular}

\section{Criteria for Passing Units 6-12:}

Student must obtain $\mathbf{1 6}$ out of 20 possible points in order to pass each C\&I unit.

$\square$ Due to the cooperative nature of the C\&I portion of this course, the instructor reserves the right to remove a student from a group if he or she repeatedly fails to contribute. Any student who is removed from a group will receive a grade of zero for Units 6-12.

IV. Clinical Lab Experiences (Units 13-20)

\begin{tabular}{|l|r|r|r|r|r|r|}
\hline \multicolumn{1}{|c|}{ Unit Name } & $\begin{array}{c}\text { Lab } \\
\text { Attend }\end{array}$ & $\begin{array}{c}\text { Lab } \\
\text { Attire }\end{array}$ & $\begin{array}{c}\text { Lab } \\
\text { Activity }\end{array}$ & $\begin{array}{c}\text { Lab } \\
\text { Reflect }\end{array}$ & $\begin{array}{c}\text { Point } \\
\text { Total }\end{array}$ & $\begin{array}{c}\text { Units } \\
\text { Passed }\end{array}$ \\
\hline Unit 13 Elementary (Lab 1) & $/ 1$ & $/ 1$ & $/ 4$ & $/ 4$ & $/ 10$ & \\
\hline Unit 14 Elementary (Lab 2) & $/ 1$ & $/ 1$ & $/ 4$ & $/ 4$ & $/ 10$ & \\
\hline Unit 15 Elementary (Lab 3) & $/ 1$ & $/ 1$ & $/ 4$ & $/ 4$ & $/ 10$ & \\
\hline Unit 16 Elementary (Lab 4) & $/ 1$ & $/ 1$ & $/ 4$ & $/ 4$ & $/ 10$ & \\
\hline Unit 17 Middle (Lab 5) & $/ 1$ & $/ 1$ & $/ 4$ & $/ 4$ & $/ 10$ & \\
\hline Unit 18 Middle (Lab 6) & $/ 1$ & $/ 1$ & $/ 4$ & $/ 4$ & $/ 10$ & \\
\hline Unit 19 Secondary (Lab 7) & $/ 1$ & $/ 1$ & $/ 4$ & $/ 4$ & $/ 10$ & \\
\hline Unit 20 Secondary (Lab 8) & $/ 1$ & $/ 1$ & $/ 4$ & $/ 4$ & $/ 10$ & \\
\hline
\end{tabular}

\section{Criteria for Passing Units 13-20:}

Student must obtain $\mathbf{8}$ out of $\mathbf{1 0}$ possible points in order to pass each lab unit.

$\square$ Student must attend every lab session prepared to participate in physical activity. Points will be deducted for lateness.

Student must dress professionally and appropriately for physical activity (i.e. gym shoes, shorts, running pants). Points will be deducted for unprofessional and/or inappropriate attire.

- Student must fully participate in the scheduled instructional activities. Points will be deducted for the failure to participate, the use of inappropriate language, and/or unprofessional conduct.

$\square$ Students must complete a daily lab reflection concerning the day's instructional activities.

$\square$ Due to the experiential nature of the lab portion of this class, students will not be allowed to make-up a missed laboratory session. 


\section{PET 198K Graphic Organizer (PETE Curriculum Design)}

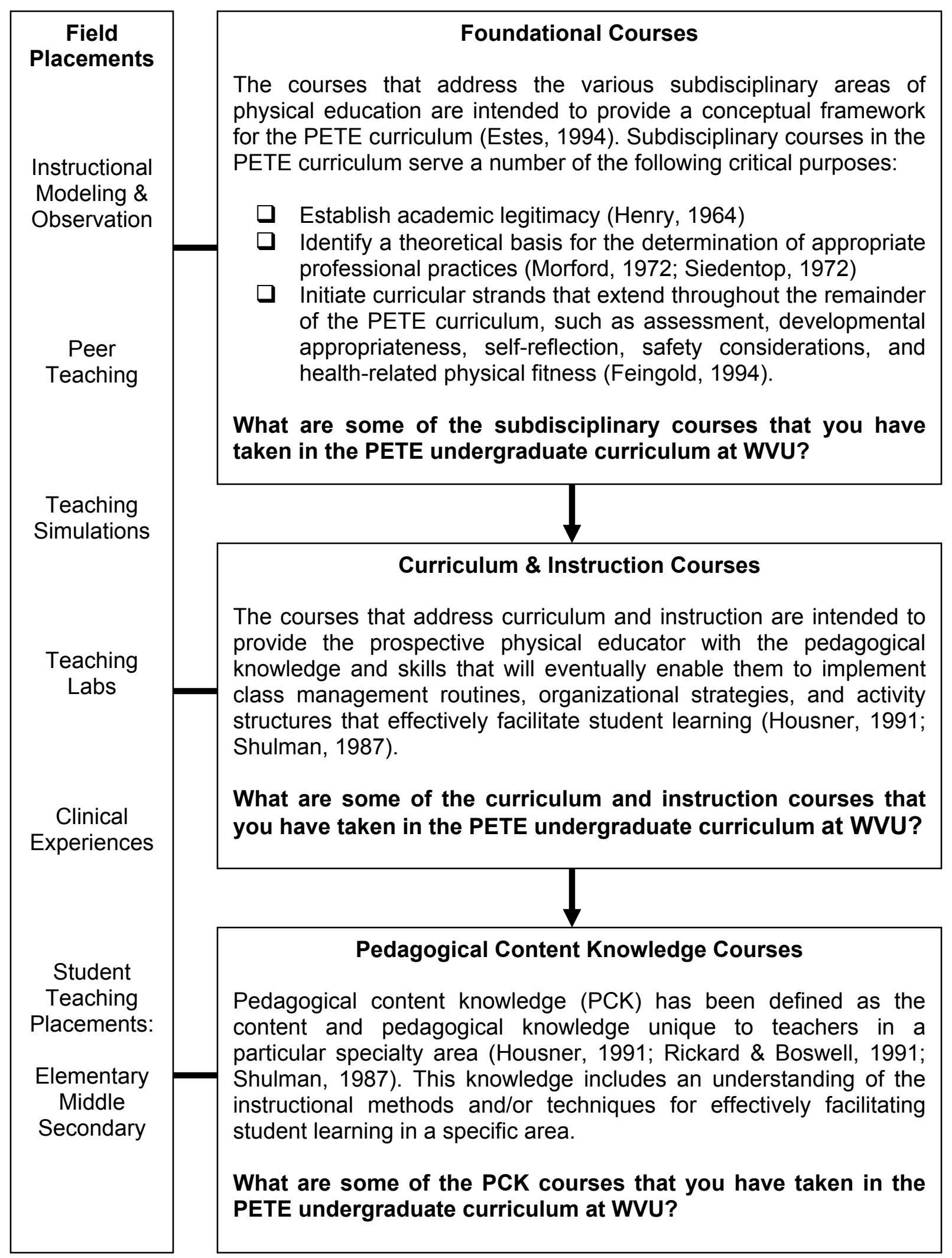




\section{PET 198K Graphic Organizer (Health-Related Physical Fitness Strand in the Revised PETE Curriculum)}

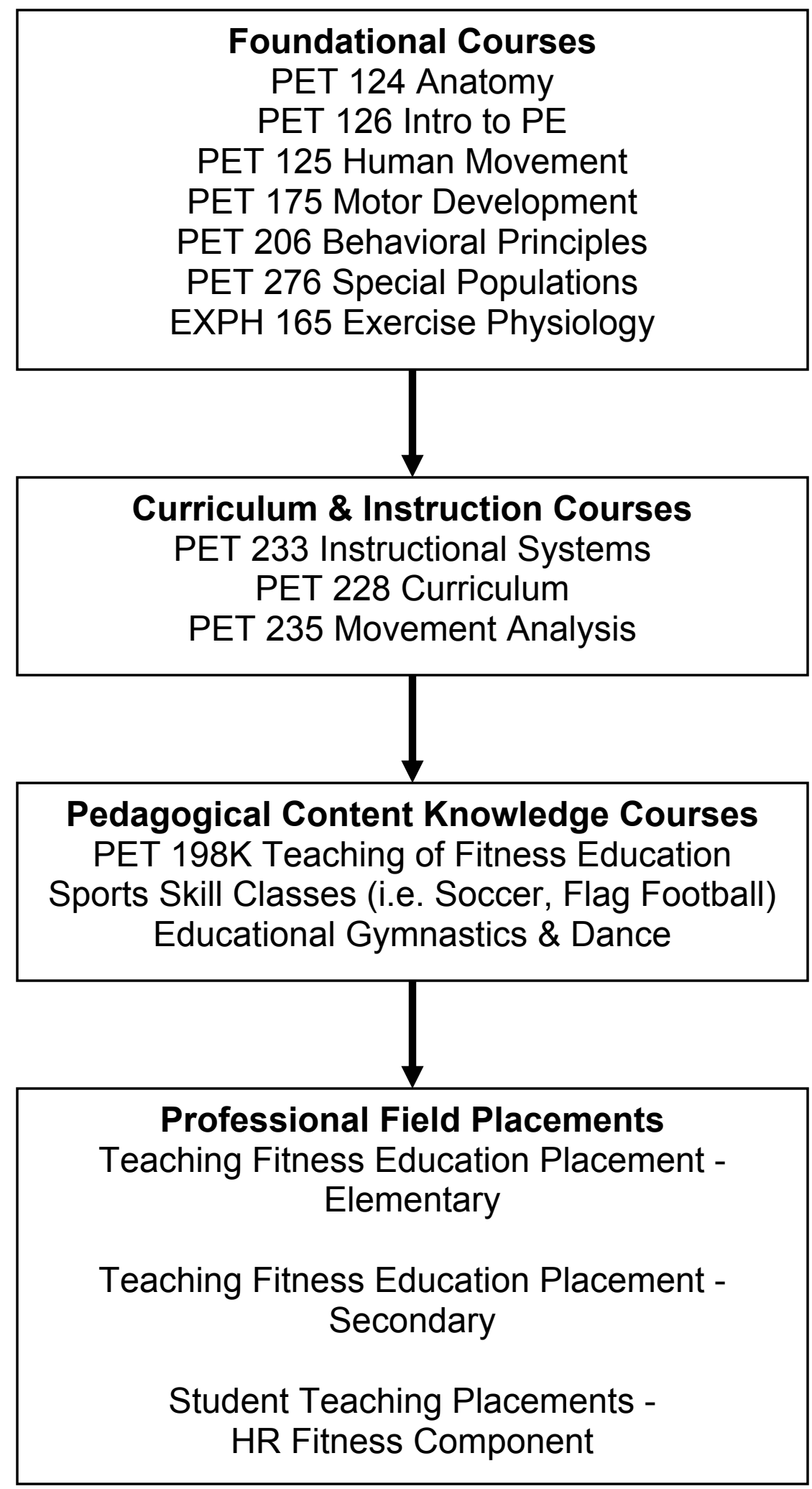




\section{PET 198K Graphic Organizer (Course Content)}

Week 1

Week 2

Week 3

Week 4

Week 5

Week 6

Week 7

Week 8

Week 9

Week 10

Week 11

Week 12

Week 13

Week 14

Week 15

Week 16
Course Orientation

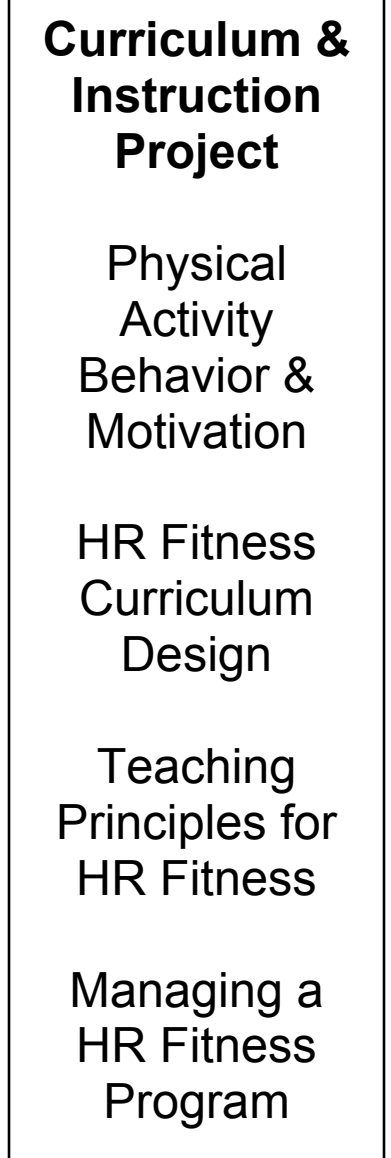

Assessing HR

Fitness

Physical Activities for Improving HR

Fitness
Clinical Lab Experiences

Elementary

Middle

Secondary 


\section{PET 198K Graphic Organizer (Course Assessment)}

\begin{tabular}{|c|c|c|c|}
\hline Week 1 & \multicolumn{3}{|c|}{ Course Orientation } \\
\hline $\begin{array}{l}\text { Week } 3 \\
\text { Week } 4\end{array}$ & $\begin{array}{l}\text { Physical } \\
\text { Activity \& } \\
\text { Fitness } \\
\text { (Units 1- 4) }\end{array}$ & $\begin{array}{c}\text { Curriculum \& } \\
\text { Instruction } \\
\text { Project } \\
\text { (Units 6-12) }\end{array}$ & \\
\hline $\begin{array}{l}\text { Week } 5 \\
\text { Week } 6\end{array}$ & $\begin{array}{l}\text { Lecture } \\
\text { Reactions }\end{array}$ & $\begin{array}{l}\text { Literature } \\
\text { Review }\end{array}$ & \\
\hline Week 7 & $\begin{array}{c}\text { Study Guide } \\
\text { Questions }\end{array}$ & $\begin{array}{l}\text { Internet } \\
\text { Search }\end{array}$ & \\
\hline $\begin{array}{l}\text { Week } 8 \\
\text { Week } 9\end{array}$ & Unit Quizzes & $\begin{array}{l}\text { Instructional } \\
\text { Materials }\end{array}$ & \\
\hline $\begin{array}{l}\text { Week } 10 \\
\text { Week } 11\end{array}$ & Quiz Retakes & $\begin{array}{c}\text { Assessment } \\
\text { Materials }\end{array}$ & \\
\hline $\begin{array}{l}\text { Week } 12 \\
\text { Week } 13\end{array}$ & & $\begin{array}{l}\text { Teaching } \\
\text { Clinic } 1 \\
\text { Teaching }\end{array}$ & $\begin{array}{l}\text { Clinical Lab } \\
\text { Experiences } \\
\text { (Units 13-20) }\end{array}$ \\
\hline $\begin{array}{l}\text { Week } 14 \\
\text { Week } 15\end{array}$ & & $\begin{array}{l}\text { Clinic } 2 \\
\text { Instructional } \\
\text { Portfolio }\end{array}$ & $\begin{array}{c}\text { Attire } \\
\text { Attendance } \\
\text { Participation } \\
\text { Reflection }\end{array}$ \\
\hline Week 16 & & $\begin{array}{l}\text { al Examinat } \\
\text { (Unit 5) }\end{array}$ & \\
\hline
\end{tabular}


Appendix E 206

\section{APPENDIX E}

Summary of Recommended

Instructional Strategies 


\section{EXSC Delphi Investigation Recommended Instructional Methods}

\section{$\underline{\text { Response Categories: }}$}
1. Learning Laboratories
2. Teacher Directed Instruction
3. Active Learning Strategies
4. Experiential Learning
5. Technology Integration
6. Alternative Curricular Model
15 responses $(21.74 \%)$
12 responses $(17.39 \%)$
22 responses $(31.88 \%)$
11 responses $(15.94 \%)$
7 responses $(10.14 \%)$
2 responses $(2.90 \%)$

\begin{tabular}{|c|c|c|}
\hline Category & Recommended Instructional Strategy & Rationale Summary \\
\hline 2 & Teacher and student demonstrations & $\begin{array}{l}\text { Students learn concepts the best by seeing. } \\
\text { Demonstration is key to every presentation. }\end{array}$ \\
\hline 3 & Hands-on practice with concepts and skills & $\begin{array}{l}\text { This method is the reason students love physical } \\
\text { education, they are able to move, touch and manipulate } \\
\text { objects. }\end{array}$ \\
\hline 3 & $\begin{array}{l}\text { Use of a physical education manual which } \\
\text { includes, history, terminology, rules, } \\
\text { regulations, safety and equipment for almost } \\
\text { all units of study }\end{array}$ & $\begin{array}{l}\text { Many students are visual learners. They need to see } \\
\text { information. This is an effective method of introducing } \\
\text { new units. }\end{array}$ \\
\hline 3 & Small group or partner work & $\begin{array}{l}\text { Change is important in the educational presentation. } \\
\text { Allowing students to become leaders and help their } \\
\text { peers creates an environment of everyone working } \\
\text { toward a common goal. }\end{array}$ \\
\hline 1 & Practical lab experiences & Hands on work helps to ensure understanding. \\
\hline 4 & Early student teaching experiences & $\begin{array}{l}\text { I feel it is important for students to see the dynamics of } \\
\text { the job early in the program to better ensure their } \\
\text { satisfaction with the curriculum and expectations. }\end{array}$ \\
\hline 2 & Classroom instruction through lecture & $\begin{array}{l}\text { Good old lecture is good for learning basics. When the } \\
\text { information is presented well and there is class } \\
\text { discussion I have learned much. }\end{array}$ \\
\hline 3 & Research and verbal presentations & $\begin{array}{l}\text { I think you retain more info when researching and } \\
\text { finding various references. Teachers also need to learn } \\
\text { public speaking and presenting material to students. } \\
\text { Being comfortable with this is very important. }\end{array}$ \\
\hline 2 & Traditional lecture format with discussion & $\begin{array}{l}\text { There are many topics such as pathophysiology, safety, } \\
\text { emergency care, human development, management, etc. } \\
\text { that I believe are most efficiently covered in a direct } \\
\text { teaching format. The advantages of lecture format - } \\
\text { factual information is presented in a logical direct } \\
\text { manner - useful for large groups and efficient delivery } \\
\text { method. Discussion allows students to question, clarify } \\
\text { and challenge. }\end{array}$ \\
\hline 1 & $\begin{array}{l}\text { Exercise physiology and exercise testing } \\
\text { lecture/lab format }\end{array}$ & $\begin{array}{l}\text { This format would include a lecture format combined } \\
\text { with a lab where students obtain the knowledge, skills } \\
\text { and abilities related to testing and screening the various } \\
\text { aspects of fitness. Provide the advantages of lecture as } \\
\text { well as create a small group opportunity for hands-on } \\
\text { learning with actual testing equipment. }\end{array}$ \\
\hline
\end{tabular}




\begin{tabular}{|c|c|c|}
\hline 1 & Exercise techniques lecture/lab & $\begin{array}{l}\text { This course would include lecture info on exercise } \\
\text { prescription and methods of teaching. The lab would } \\
\text { include hands on teaching of group exercise as well as } \\
\text { techniques to teach individuals how to work on the } \\
\text { health-related fitness components. Again a combo of } \\
\text { lecture as well as hands on. Student would be required } \\
\text { to teach group exercise to their peers and be able to } \\
\text { demonstrate the KSAs related to workshop with } \\
\text { individual on their fitness (motivation, proper spotting, } \\
\text { progression, etc.). }\end{array}$ \\
\hline 3 & Role playing with peers & $\begin{array}{l}\text { Role playing with peers allows students to practice } \\
\text { skills }\end{array}$ \\
\hline 3 & Guest Speakers & $\begin{array}{l}\text { Guest speakers from community provide real life } \\
\text { situations for discussion }\end{array}$ \\
\hline 4 & Observation of PE settings & $\begin{array}{l}\text { Observations of real PE classes will reinforce info and } \\
\text { provide new insight }\end{array}$ \\
\hline 2 & Lecture and discussion & Efficient - will cover all necessary topics. \\
\hline 1 & $\begin{array}{l}\text { Lab-based coursework regarding "personal" } \\
\text { fitness followed by "professional" level } \\
\text { where they learn scientific principles in more } \\
\text { detail }\end{array}$ & Students need opportunities with active learning. \\
\hline 4 & $\begin{array}{l}\text { Guided field experiences and internships } \\
\text { throughout curriculum }\end{array}$ & $\begin{array}{l}\text { Students will learn more PE by being in a PE setting. } \\
\text { The feedback from a teacher will help them learn more } \\
\text { effectively from experience. The field-based learning } \\
\text { should be done both early in their program and later so } \\
\text { that students can see application to their coursework. }\end{array}$ \\
\hline 5 & $\begin{array}{l}\text { Learn how to use multi-media content } \\
\text { delivery - PowerPoint }\end{array}$ & The wave of the future - or actually the present. \\
\hline 1 & Exercise physiology lab classes & $\begin{array}{l}\text { Students need to learn various and appropriate fitness } \\
\text { testing protocols and be aware of scientific attributes of } \\
\text { a test. }\end{array}$ \\
\hline 5 & $\begin{array}{l}\text { Learn to use HR monitors thru different } \\
\text { activity sessions }\end{array}$ & $\begin{array}{l}\text { To learn the value of measuring the intensity of } \\
\text { exercise. }\end{array}$ \\
\hline 1 & $\begin{array}{l}\text { Lab sessions regarding strength/conditioning } \\
\text { exercise techniques }\end{array}$ & Importance of this component of fitness. \\
\hline 2 & Lecture format & $\begin{array}{l}\text { Economic way to deliver content, evaluate knowledge } \\
\text { of descriptive/definitional information, and screen out } \\
\text { those who can't handle that. }\end{array}$ \\
\hline 1 & Lab format & $\begin{array}{l}\text { Hands-on experience that parallel lecture content. This } \\
\text { allows assessment of quantitative skills, ability to } \\
\text { interpret data, and draw appropriate conclusions. }\end{array}$ \\
\hline 3 & Practice teaching activities & $\begin{array}{l}\text { Must be able to deliver the goods, comfortably and with } \\
\text { confidence, in front of students. }\end{array}$ \\
\hline 4 & Practicum/Internship & $\begin{array}{l}\text { To get real world experience in a natural (schools, } \\
\text { fitness club, etc.) environment. Opportunity to work } \\
\text { with a professional and learn to be a professional. }\end{array}$ \\
\hline 1 & Hands on lab experiences & $\begin{array}{l}\text { Not only have students evaluate performances and skills } \\
\text { testing - have students perform tasks. Laboratory } \\
\text { experiences most likely are better remembered than } \\
\text { lectures. }\end{array}$ \\
\hline 5 & $\begin{array}{l}\text { Use of Internet and texts to perform 3-day } \\
\text { dietary analysis }\end{array}$ & $\begin{array}{l}\text { Allows students to find links that provide information to } \\
\text { be used in teaching. }\end{array}$ \\
\hline 3 & Prepare pre-lecture/pre-lab study questions & Requires students to be prepared. \\
\hline 4 & $\begin{array}{l}\text { Field trips/observations at EXPHS sites that } \\
\text { perform evaluations/rehabilitation }\end{array}$ & Student sees (observes) class material in the real world. \\
\hline
\end{tabular}




\begin{tabular}{|c|c|c|}
\hline 2 & Lecture & Students respond to receiving material in lecture format. \\
\hline 1 & Hands-on labs & $\begin{array}{l}\text { Some students respond better when they can be active } \\
\text { in the learning process. }\end{array}$ \\
\hline 3 & Reading assignments & $\begin{array}{l}\text { Some students need to read and review the material } \\
\text { many times. }\end{array}$ \\
\hline 2 & Question \& answer sessions & Some students are capable of synthesizing information. \\
\hline 5 & Use PowerPoint presentations & $\begin{array}{l}\text { Includes both audio and visual learning styles. } \\
\text { PowerPoint can quickly and easily be adapted to new } \\
\text { material (modify-delete). Presentation can be made very } \\
\text { attractive and interesting. }\end{array}$ \\
\hline 1 & Laboratory demonstration & $\begin{array}{l}\text { Viewing principles applied personally usually illustrates } \\
\text { quickly and easily content to be comprehended... and } \\
\text { reinforces principle discussed in PowerPoint. }\end{array}$ \\
\hline 1 & Laboratory experience & $\begin{array}{l}\text { Principles to be understood and acquired are usually } \\
\text { accomplished at the highest level with a hands-on } \\
\text { approach. }\end{array}$ \\
\hline 3 & $\begin{array}{l}\text { Prospective teachers share with others } \\
\text { (demos, teaching, leading) }\end{array}$ & $\begin{array}{l}\text { The highest level of learning is the ability to share with } \\
\text { others. This should be demonstrated prior to the person } \\
\text { (teacher) be granted permission (certification) to teach. }\end{array}$ \\
\hline 5 & Internet assisted instruction & $\begin{array}{l}\text { 1. More student involvement in their own learning, } 2 \text {. } \\
\text { Higher level of understanding possible, } 3 \text {. Learning can } \\
\text { be asynchronous. }\end{array}$ \\
\hline 4 & $\begin{array}{l}\text { Experiential learning (formal lab } \\
\text { experiments, practicum, internships) }\end{array}$ & $\begin{array}{l}\text { 1. Promotes more active involvement in learning, and } 2 \text {. } \\
\text { Enables development of practical applied skills of } \\
\text { exercise testing, Rx, and leadership. }\end{array}$ \\
\hline 3 & $\begin{array}{l}\text { Small group discussion/action to arrive at } \\
\text { learning consensus (w/ instructor guidance) }\end{array}$ & $\begin{array}{l}\text { 1. Active involvement in learning, 2. Promotes collegial } \\
\text { interaction, and 3. takes advantage of skills/knowledge } \\
\text { of fellow students }\end{array}$ \\
\hline 3 & Case study and analysis & $\begin{array}{l}\text { 1. More like real life situations, } 2 \text {. promotes integration } \\
\text { of knowledge from various disciplines, and 3. promotes } \\
\text { critical thinking. }\end{array}$ \\
\hline 3 & Group collaboration/cooperative learning & $\begin{array}{l}\text { To show in depth analysis w/ a peer group. Ability to } \\
\text { teach peers and determine project results. }\end{array}$ \\
\hline 3 & $\begin{array}{l}\text { Designing and using fitness/skill } \\
\text { stations/learning centers } \mathrm{w} / \text { modifications for } \\
\text { various levels }\end{array}$ & $\begin{array}{l}\text { Experience center design and why exercises/learning } \\
\text { experiences are chosen to fit the intended group. }\end{array}$ \\
\hline 2 & Direct instruction/demonstration/feedback & $\begin{array}{l}\text { To teach specific exercises and use of special } \\
\text { equipment (e.g., exercise balls, simple weights) and } \\
\text { other common equipments that might be found in } \\
\text { schools. }\end{array}$ \\
\hline 3 & Peer coaching & To teach one another and show deeper learning. \\
\hline 2 & $\begin{array}{l}\text { Direct Instruction (lecture-recitation-testing } \\
\text { w/ frequency) }\end{array}$ & $\begin{array}{l}\text { Most efficient and documented method of learning } \\
\text { well-established content. }\end{array}$ \\
\hline 1 & Laboratory & $\begin{array}{l}\text { Need experimental context to understand exercise } \\
\text { science principles. }\end{array}$ \\
\hline 4 & Clinical practice & $\begin{array}{l}\text { Need to effectively apply context to real children and } \\
\text { youth. }\end{array}$ \\
\hline 3 & Problem-based learning & $\begin{array}{l}\text { This enhances critical thinking skills that apply to the } \\
\text { K-12 physical education classroom setting well. }\end{array}$ \\
\hline 3 & Divergent -Discovery Teaching Style & $\begin{array}{l}\text { This gets students to think and prioritize solutions } \\
\text { according to given situations. }\end{array}$ \\
\hline 3 & $\begin{array}{l}\text { Individual or small group projects } \\
\text { emphasizing a systematic process }\end{array}$ & $\begin{array}{l}\text { It emphasizes the need to understand current, cutting } \\
\text { edge information and apply theoretical concepts } \\
\text { according to individualized interests. }\end{array}$ \\
\hline 3 & Mastery Learning approach & It puts responsibility on the student to decide the \\
\hline
\end{tabular}




\begin{tabular}{|c|c|c|}
\hline & & $\begin{array}{l}\text { importance of learning the material and the extrinsic } \\
\text { motivation (carrot) associated with it. }\end{array}$ \\
\hline 6 & $\begin{array}{l}\text { Merge EXPHS with a methods of teaching } \\
\text { course }\end{array}$ & $\begin{array}{l}\text { I recommend this method so that students learn to } \\
\text { actually apply the EXPHS concepts in a } \\
\text { classroom/gymnasium setting. In addition students } \\
\text { would learn how to plan and deliver a fitness course. }\end{array}$ \\
\hline 1 & Have EXPHS course with an applied lab & $\begin{array}{l}\text { Most physiology labs are conducted in actual human } \\
\text { performance lab, in this case, the lab would be the } \\
\text { gymnasium or field. }\end{array}$ \\
\hline 6 & $\begin{array}{l}\text { Develop an EXPHS course specifically for } \\
\text { PETE majors - developmental EXPHS }\end{array}$ & $\begin{array}{l}\text { Most EXPHS courses are focused toward adult } \\
\text { physiology. PETE students need information about } \\
\text { children and youth. }\end{array}$ \\
\hline 2 & $\begin{array}{l}\text { Didactic (lecture), directed confrontational } \\
\text { approach, coupled with ample student } \\
\text { interaction in classroom possibly } \\
\text { supplemented by instructional (PETE) lab }\end{array}$ & $\begin{array}{l}\text { The methodology, if conducted properly with a well } \\
\text { trained specialist, has been effective over the years and } \\
\text { has been, and continues to be, the dominant method of } \\
\text { disseminating knowledge (content) to interested, ready- } \\
\text { to-learn, learners. }\end{array}$ \\
\hline 4 & $\begin{array}{l}\text { Internships directed by competent leaders in } \\
\text { real world setting }\end{array}$ & $\begin{array}{l}\text { University internships, as a method of learning, when } \\
\text { coupled with adjunctive instruction, appears to be a } \\
\text { contemporary method allowing students 'hands-on } \\
\text { experiences' within job setting, i.e., the marketplace. It } \\
\text { represents an instructional vehicle long talked about but } \\
\text { more recently implemented to supplement classroom } \\
\text { instruction. Learning tends to be more realistic, more } \\
\text { meaningful and more effective toward launching young } \\
\text { learners into an environment that may otherwise be } \\
\text { unknown, untested or even threatening. }\end{array}$ \\
\hline 5 & Learning via Internet - use of technology & $\begin{array}{l}\text { Accessibility to and competence with current } \\
\text { technology is a basic phenomenon as young students } \\
\text { begin their education in contemporary America. Online } \\
\text { information, availability of e-resources and distance } \\
\text { learning via the web present an enormous vehicle for } \\
\text { learning. Couple with routine computer laboratories, } \\
\text { power-point availability, etc. no longer can the } \\
\text { instructor limit her/his teaching station to the classroom } \\
\text { using chalk and overheads. Through technology, the } \\
\text { opportunities are both exciting and challenging. }\end{array}$ \\
\hline 4 & $\begin{array}{l}\text { Cooperative learning blending school } \\
\text { environment with university environment }\end{array}$ & $\begin{array}{l}\text { Learning should be more effective when learners take } \\
\text { their knowledge and early expertise out into the school } \\
\text { setting and "try it on for size. This serves a duel role: } \\
\text { better programs in physical education for the school } \\
\text { children and greater learning opportunities for the } \\
\text { university students. The partnership is greater and the } \\
\text { results are more forthcoming than otherwise. }\end{array}$ \\
\hline 5 & Dynamic multimedia presentation of content & $\begin{array}{l}\text { Students must first know the basic facts and concepts. } \\
\text { Presenting the information in a dynamic way using } \\
\text { multiple sources of media assists students learn this } \\
\text { information. }\end{array}$ \\
\hline 3 & $\begin{array}{l}\text { Problem solving/case studies/small group } \\
\text { sessions }\end{array}$ & $\begin{array}{l}\text { Once the students understand the basic facts and } \\
\text { concepts they must engage with the content. These } \\
\text { methods promote discussion and expose students to a } \\
\text { variety of different perspectives. }\end{array}$ \\
\hline 4 & Practicum/field experiences & $\begin{array}{l}\text { Ultimately for students to master this content they must } \\
\text { have an opportunity to apply it under the supervision of } \\
\text { a certified professional. }\end{array}$ \\
\hline
\end{tabular}


Appendix E 211

\begin{tabular}{|c|l|l|}
\hline $\mathbf{2}$ & Lecture/discussion & Effective if large course if carefully prepared \\
\hline $\mathbf{1}$ & $\begin{array}{l}\text { Lab exercises - collect analyze/interpret data } \\
\text { and write lab reports }\end{array}$ & Hands-on promotes critical thinking and active learning \\
\hline $\mathbf{3}$ & $\begin{array}{l}\text { Guide them to promote understanding of } \\
\text { control and mechanisms more than learning } \\
\text { facts }\end{array}$ & $\begin{array}{l}\text { If they have super understanding of mechanisms and } \\
\text { how things work, they are much more skillful in self- } \\
\text { initiated application }\end{array}$ \\
\hline $\mathbf{1}$ & Exercise Physiology Lab & $\begin{array}{l}\text { Prospective physical educators can learn and apply } \\
\text { knowledge in a lab setting. Students can analyze, } \\
\text { synthesize and evaluate exercise science information } \\
\text { and methods. Learn practical application. Students see } \\
\text { first hands effects of training methods and experience. }\end{array}$ \\
\hline $\mathbf{2}$ & Lecture & $\begin{array}{l}\text { Basic information can be quickly and effectively taught } \\
\text { this way. Written tests can identify if students have } \\
\text { basic understanding. }\end{array}$ \\
\hline $\mathbf{4}$ & $\begin{array}{l}\text { Fieldwork with athletic coaches or } \\
\text { exemplary physical educators }\end{array}$ & $\begin{array}{l}\text { Prospective educators see the importance, value, and } \\
\text { relevance of exercise science in their field. Either with } \\
\text { competitive athletes or with the general public school } \\
\text { student population (K-12). }\end{array}$ \\
\hline $\mathbf{3}$ & $\begin{array}{l}\text { Prospective physical educators read and } \\
\text { review recent research findings and studies }\end{array}$ & $\begin{array}{l}\text { Students see an evolving field - students learn resources } \\
\text { for up to data research. }\end{array}$ \\
\hline
\end{tabular}




\section{CURRICULUM VITAE}




\section{Sean M. Bulger \\ University of Wisconsin-Eau Claire \\ Department of Kinesiology \& Athletics \\ bulgersm@uwec.edu}

\section{EDUCATION:}

(2004) West Virginia University, Morgantown, WV

Doctor of Education in Physical Education Teacher Education

Cognate Area: Applied Exercise Science

Dissertation Topic: Exercise Science in Physical Education Teacher Education

1997 North Dakota State University, Fargo, ND

Master of Education in Secondary Education

Cognate Area: Physical Education/Athletic Administration

1992 Canisius College, Buffalo, NY

Bachelor of Science in Physical Education

\section{QUALIFICATIONS:}

Advising

- Represented department at various on-campus events related to academic advising

- Initiated electronic advising update for exercise management and exercise science students

- Collaborated with career services to establish an ongoing professional development initiative

- Advised between 60 to 110 undergraduate students per semester

\section{Scholarship}

- Presented seventeen scholarly papers at state, regional, and national conferences

- Published eight refereed manuscripts in the areas of children's physical activity programming and the scholarship of teaching within higher education

- Conducted regular programmatic evaluation on teaching effectiveness and curricular design

- Established skill in using word processing, spreadsheet, presentation, statistical, and webbased instructional software

Service

- Provided leadership to departmental, college, and university committees

- Maintained active membership within multiple professional organizations

- Directed a community-based physical fitness education program for school-aged children

- Designed, implemented, and evaluated strength and conditioning programs for studentathletes within the context of an NCAA Division II athletic program

\section{Teaching}

- Taught undergraduate professional development courses in exercise science, exercise management, and physical education teacher education

- Supervised student teaching and internship placements

- Obtained consistently high student and peer teaching evaluations

- Developed excellent problem solving and communication skills 


\section{COURSES TAUGHT:}

Exercise Management \& Exercise Science Courses

- Introduction to Exercise Management/Science

- Essentials of Strength Training

- Essentials of Cardiovascular Training

- Program Design and Development

- Applied Fitness Activities

Physical Education Teacher Education Courses

- Exercise for School-Aged Children

- Motor Behavior Issues Seminar

- Teaching of Physical Fitness Education

- Teaching of Flag Football

- Teaching of Non-Traditional Sports/Activities

Professional Field Placements

- Adapted Physical Education Clinic

- Exercise Management/Science Internship

- Fitness Education Practicum

- Student Teaching Placement

\section{PROFESSIONAL AFFILIATIONS \& RELATED ACTIVITIES:}

American Alliance for Health, Physical Education, Recreation, \& Dance (AAHPERD)

- Member of AAHPERD Research Consortium

- Member of National Association for Sport \& Physical Education (NASPE)

- Presenter at numerous AAHPERD and NASPE conferences

- Co-author of NASPE assessment manuscript on sport education

- Reviewer of research abstracts for the 2003 and 2004 AAHPERD National Convention

- Section reviewer for the journal titled Teaching Elementary Physical Education

American College of Sports Medicine (ACSM)

- Member of the Alliance of Health and Fitness Professionals

- Certified in Adult CPR/AED by the American Red Cross

National Strength \& Conditioning Association (NSCA)

- Certified Strength \& Conditioning Specialist (CSCS) since 1993

- Recipient of the 1999 NSCA Challenge Scholarship for graduate students

- Program Sponsor and Director for NSCA Educational Recognition Program

- Member of NSCA Speaker Resource Network

- Script writer for NSCA functional anatomy/biomechanics videotape

- Assistant director for 1996 National Strength \& Speed Camp/Coaching Clinic

- Strength \& conditioning consultant for various youth sports camps and clinics

North American Society for Pediatric Exercise Medicine (NASPEM) 


\section{EMPLOYMENT HISTORY:}

\author{
2000-Present Assistant Professor \\ 1999-2000 Graduate Teaching Assistant \\ 1997-1999 Graduate Teaching Assistant \\ 1995-1997 \\ 1995 \\ 1994-1995 \\ 1992-1994 \\ 1991-1992
}

University of Wisconsin-Eau Claire, Eau Claire, WI

WVU-Motor Development Center, Morgantown, WV

WVU-School of Physical Education, Morgantown, WV

North Dakota State University, Fargo, ND

Johnson \& Johnson HCSM, Buffalo, NY

State University of New York at Buffalo, Buffalo, NY

Tonawanda Aquatic \& Fitness Center, Tonawanda, NY

St. Aloysius Gonzaga Elementary School, Buffalo, NY

\section{UNIVERSITY COMMITTEES:}

2003-Present

2003 Fall

2003 Spring

2002-2003

2002-2003

$2000-2003$

2000-2003

2001-2002

2001-2002

2001 Summer

2000-2002
Curriculum \& Assessment*

Faculty Advisory Board

Search \& Screen Committee

Scholarship Committee

Technology Committee

Assessment Committee**

Nominating Committee

Search \& Screen Committee

Search \& Screen Committee***

Portfolio Assessment

Curriculum Committee
UW-Eau Claire Department of Kinesiology \& Athletics UW-Eau Claire Career Services

UW-Eau Claire Department of Kinesiology \& Athletics UW-Eau Claire Department of Kinesiology \& Athletics UW-Eau Claire Department of Kinesiology \& Athletics UW-Eau Claire Department of Kinesiology \& Athletics UW-Eau Claire Department of Kinesiology \& Athletics UW-Eau Claire Department of Kinesiology \& Athletics UW-Eau Claire College of Professional Studies UW-Eau Claire Assessment Committee UW-Eau Claire Department of Kinesiology \& Athletics

* Restructuring of committees within the Department of Kinesiology \& Athletics

**Served as Chair for the Department of Kinesiology \& Athletics Assessment Committee from 2000-2003

*** Served as Chair for the College of Professional Studies Associate Dean Search \& Screen Committee

\section{HONORS \& AWARDS:}

2002-2003

2001-2002

2000-2001

1999

1999

1997

1997
Meritorious Performance

Outstanding Teaching \& Merit

Outstanding Teaching

Challenge Scholarship

Honor Society Membership

Honor Society Membership

Graduate Student of the Year
University of Wisconsin-Eau Claire

University of Wisconsin-Eau Claire

University of Wisconsin-Eau Claire

National Strength \& Conditioning Association

West Virginia University - Gamma Beta Phi

North Dakota State University - Phi Kappa Phi

North Dakota State University - Department of HPER

\section{FUNDING HISTORY}

2003 Fall Student/Faculty Research Grant $\$ 1250$ from University of Wisconsin-Eau Claire

2003 Fall Faculty Travel Support Grant

2003 Summer Staff Development Grant

2003 Summer Differential Tuition Award

2003 Spring

2002 Summer

2001 Spring

2000 Summer

1999 Fall

1998 Fall
Faculty Travel Support Grant

Differential Tuition Award

Small Research Project Grant

Student Travel Support Grant

Student Travel Support Grant

Student Travel Support Grant
\$1065 from University of Wisconsin-Eau Claire

\$1316 from University of Wisconsin-Eau Claire

\$3667 from University of Wisconsin-Eau Claire

$\$ 967$ from University of Wisconsin-Eau Claire

$\$ 2000$ from University of Wisconsin-Eau Claire

$\$ 500$ from University of Wisconsin-Eau Claire

$\$ 450$ from West Virginia University

$\$ 400$ from West Virginia University

$\$ 500$ from West Virginia University 


\section{SCHOLARLY PUBLICATIONS:}

Bulger, S.M. (2002). Making sense of physical activity guidelines. The School News, 7(2), 19.

Bulger, S.M. (2002). Aerobic and anaerobic exercise. The School News, 6(7), 18.

Bulger, S.M. (2001). Selecting an exercise facility. The School News, 6(3), 19.

Bulger, S.M., Mohr, D.J., Carson, L.M., Robert, D.L., \& Wiegand, R.L. (2000). Preparing prospective physical educators in exercise physiology. Quest, 52, 166-185. (Refereed paper)

Bulger, S.M., Hawkins, A.H., \& Mohr, D.J. (2002). Behavioral course design and student learning. Effective Teaching [Online], 6(1). Available at the following website http://cte.uncwil.edu/et/articles.htm [2002, September 3]. (Refereed paper)

Bulger, S.M., Mohr, D.J, Carson, L.M, \& Wiegand, R.L. (2001). Infusing health-related physical fitness in physical education teacher education. Quest, 53, 403-417. (Refereed paper)

Bulger, S.M., Mohr, D.J., Graves, M.A., \& Wiegand, R.L. (2000). The effectiveness of traditional, theory based versus alternative, pedagogical content knowledge based exercise physiology courses at delivering exercise physiology content knowledge to physical education teacher education students. Research Quarterly for Exercise \& Sport, 71 (Suppl. 1), A-66. (Refereed abstract)

Bulger, S.M., Mohr, D.J., \& Walls, R.T. (2002). Stack the deck in favor of your students by using the four aces of effective teaching. Effective Teaching [Online], 5(2). Available at the following website http://cte.uncwil.edu/et/articles.htm [2002, May 7]. (Refereed paper)

Bulger, S.M., Mohr, D.J., \& Wiegand, R.L. (2003). Factors effecting student learning in traditional, theory based versus alternative, pedagogical content knowledge based exercise physiology courses: An explanatory study. Research Quarterly for Exercise \& Sport, 73 (Suppl. 1), A-38. (Refereed abstract)

Bulger, S.M., Townsend, J.S., \& Carson, L.M. (2001). Promoting responsible student decision-making in elementary physical education. Journal of Physical Education, Recreation \& Dance, 72(7), 18-23. (Refereed paper)

Mohr, D.J., Townsend, J.S., \& Bulger, S.M. (2001). A pedagogical approach to sport education season planning. Journal of Physical Education, Recreation \& Dance, 72(9), 37-46. (Refereed paper)

Mohr, D.J., Townsend, J.S., \& Bulger, S.M. (2002). Maintaining the PASE: A day in the life of sport education. Journal of Physical Education, Recreation \& Dance, 73(1), 36-44. (Refereed paper)

Townsend, J.S., Mohr, D.J, Rairigh, R.M., \& Bulger, S.M. (2003). Assessment within a sport education season. Reston, VA: NASPE Publications. (Refereed manuscript)

\section{SCHOLARLY PRESENTATIONS:}

Bulger, S.M., Carson, L.M., Mohr, D.J., \& Wiegand, R.L. (2000, July). The infusion of a health-related fitness strand into the physical education teacher education curriculum. Paper presented at the National Conference for K-12 Physical Educators of the National Association for Sport and Physical Education (NASPE), Baltimore, MD. (Refereed presentation) 
Bulger, S.M., Cluphf, D., Housner, L.D., \& Wilmoth, B. (1998, October). Evaluating the basic instruction program: Triangulating data on program effectiveness. Paper presented at the National Conference for Promoting Lifelong Physical Activity of the National Association for Sport and Physical Education (NASPE), Salt Lake City, Utah. (Refereed presentation)

Bulger, S.M., Graves, M.A., Mohr, D.J., \& Townsend, J.S. (1999, November). Inte"great"ion. Paper presented at the State Conference of the Illinois Association for Health, Physical Education, Recreation and Dance (IAHPERD), Arlington Heights, IL. (Refereed presentation)

Bulger, S.M., Mohr, D.J., Graves, M.A., \& Wiegand, R.L. (2000, March). The effectiveness of traditional, theory based versus alternative, pedagogical content knowledge based exercise physiology courses at delivering exercise physiology content knowledge to physical education teacher education students. Poster session presented at the Annual Convention of the American Alliance for Health, Physical Education, Recreation and Dance (AAHPERD), Orlando, FL. (Refereed presentation)

Bulger, S.M., Mohr, D.J., Graves, M.A., \& Wiegand, R.L. (1999, October). Preparing physical education teacher educators to be subdisciplinary specialists. Paper presented at the National Teacher Education Conference of the National Association for Sport and Physical Education (NASPE), Bloomingdale, IL. (Refereed presentation)

Bulger, S.M., Mohr, D.J., \& Wiegand, R.L. (2003, April). Factors effecting student learning in traditional, theory-based versus alternative, pedagogical content knowledge-based exercise physiology courses: An explanatory study. Poster session presented at the Annual Convention of the American Alliance for Health, Physical Education, Recreation, and Dance (AAHPERD), Philadelphia, PA. (Refereed presentation)

Carson, L.M., \& Bulger, S.M. (2000, July). Description of an after-school fitness education program for school-aged children. Paper presented at the National Conference for K-12 Physical Educators of the National Association for Sport and Physical Education (NASPE), Baltimore, MD. (Refereed presentation)

Carson, L.M., Bulger, S.M., \& Townsend, J.S. (2000, July). Enhance fitness levels by collaborating with classroom teachers: A middle school's success story. Paper presented at the National Conference for K-12 Physical Educators of the National Association for Sport and Physical Education (NASPE), Baltimore, MD. (Refereed presentation)

Graves, M.A., Bulger, S.M., Mohr, D.J., \& Wiegand, R.L. (1999, October). Description of an innovative curricular model for use in PETE. Paper presented at the National Teacher Education Conference of the National Association for Sport and Physical Education (NASPE), Bloomingdale, IL. (Refereed presentation)

Graves, M.A., Mohr, D.J., Bulger, S.M., \& Townsend, J. S. (1999, November). Sport education workshop. Paper presented at the State Conference of the Illinois Association for Health, Physical Education, Recreation and Dance (IAHPERD), Arlington Heights, IL. (Refereed presentation)

Graves, M.A., Mohr, D.J., Bulger, S.M., \& Wiegand, R.L. (1999, February). Techniques for teaching the sports education curriculum model to pre-service physical education teachers. Paper presented at the Midwest District Conference of the American Alliance for Health, Physical Education, Recreation and Dance (AAHPERD), Huntington, WV. (Refereed presentation) 
Housner, L.D., \& Bulger, S.M. (1999, February). Administrative roundtable. Paper presented at the Midwest District Conference of the American Alliance for Health, Physical Education, Recreation and Dance (AAHPERD), Huntington, WV. (Refereed presentation)

Housner, L.D., Treanor, L., Bulger, S.M., \& Liang, G. (1999, April). Managerial task structures and instructional climate: A study of beginning teachers, parents, and physical education teachers. Paper presented at the Annual Convention of the American Alliance for Health, Physical Education, Recreation and Dance (AAHPERD), Boston, MA. (Refereed presentation)

Lindauer, J., Bulger, S.M., \& Jacobsen, B. (2003, October). The role of professional development in physical education teacher education. Poster session presented at the National Teacher Education Conference of the National Association for Sport and Physical Education (NASPE), Baton Rouge, LA. (Refereed presentation)

Mohr, D.J., Bulger, S.M., Graves, M.A., \& Wiegand, R.L. (1999, October). A systematic strategy for assessing PETE programs. Paper presented at the National Teacher Education Conference of the National Association for Sport and Physical Education (NASPE), Bloomingdale, IL. (Refereed presentation)

Robert, D.L., Bulger, S.M., Mohr, D.J., \& Wiegand, R.L. (1999, February). Preparing prospective physical educators in exercise physiology. Paper presented at the Midwest District Conference of the American Alliance for Health, Physical Education, Recreation and Dance (AAHPERD), Huntington, WV. (Refereed presentation)

Towensend, J.S., Wiegand, R.L., Bulger, S.M., Mohr, D.J., \& Metzler, M. (2002, April). Curricular issues in physical education teacher education. Poster session presented at the Annual Convention of the American Alliance for Health, Physical Education, Recreation and Dance (AAHPERD), San Diego, CA. (Refereed presentation) 